\title{
Identification of VMS ore lens reflections using vertical seismic profiling and 3D finite difference modeling in Flin Flon, Manitoba, Canada.
}

by

\author{
David Melanson \\ B.Sc (Hons.) Earth Sciences - Carleton University, 2012
}

A thesis submitted to the Faculty of Graduate and Postdoctoral Affairs in partial fulfillment of the requirements for the degree of

Master of Science

in

Earth Sciences

Carleton University

Ottawa, Ontario

(C) 2014

David Melanson 


\begin{abstract}
In October 2006, three-component multi-offset vertical seismic profile (VSP) data were acquired from three deviated wells in the Flin Flon, Manitoba, Canada mining camp using dynamite and vibroseis sources. These VSP data were processed to identify reflections originating from the 85.5 Mt Flin Flon-Callinan-777 VMS ore system. From drill records, mine plans, surficial maps and seismic data, 3D voxel models of the local geology and known ore lenses were built, which were then used in 3D finite differencemodeled simulations of the VSP surveys. The number of geological units partitioning the models were increased incrementally to study the effects on seismic response of massive sulphide ore and major rock units. The simulations and field data were jointly visualized and the reflection signature of the known VMS ore lenses in the field VSP data was identified and characterized.
\end{abstract}




\section{Acknowledgements}

First and foremost, I would like to thank my supervisors Dr. Don White and Dr. Claire Samson of the Geological Survey of Canada (GSC) and Carleton University for their guidance and expertise during this project. Their enthusiasm, commitment and patience has been constant throughout the past two years, and has contributed greatly toward the success of this project. Hudbay Minerals Inc. provided a significant amount of data used in this project.

I would like to sincerely thank Dr. Gilles Bellefleur and Dr. Ernst Schetselaar at the GSC for their assistance with VSP data processing and 3D finite difference modeling. Dr. Doug Schmitt of the University of Alberta assisted with the field data acquisition and preprocessing. Brian Roberts and Azad Rafeek at the GSC provided support with software, the computer cluster and document printing. All of these people were available anytime I had a request and were tremendously helpful. Jim Hunter, Pascal Audet and Brian Cousens of my examination committee also made contributions to this thesis.

Many fellow students at Carleton University and the GSC provided input and countless productive discussions. Specifically, Steve Crane, Kyle Harris, Becki Montsion, Brian Bancroft, Carolyn Dziawa, Jen Blanchard, Laura Olson and Erika Anderson each contributed significantly. Last but certainly not least, I'd like to thank my family and friends for their unwavering support and interest. 


\section{Table of contents}

Title page

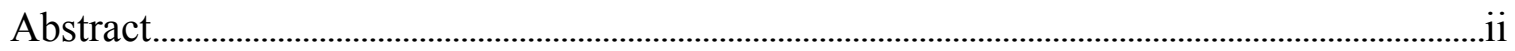

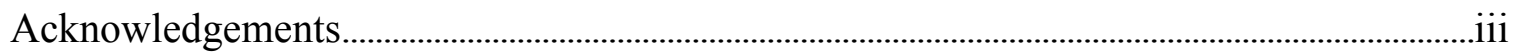

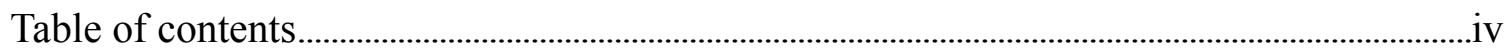

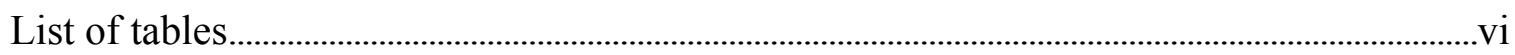

List of figures....................................................................................................................................vii

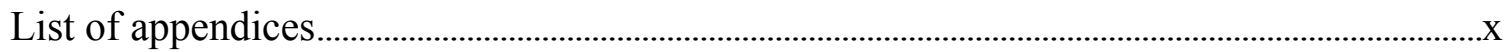

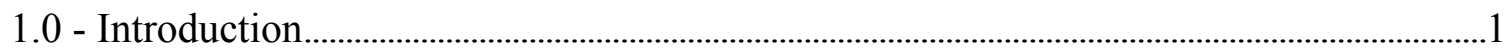

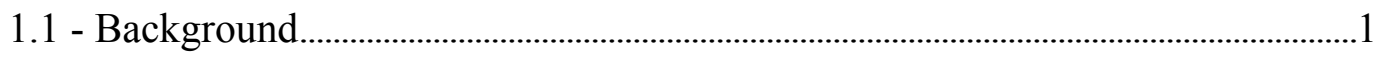

1.2 - Objectives and approach...................................................................................2

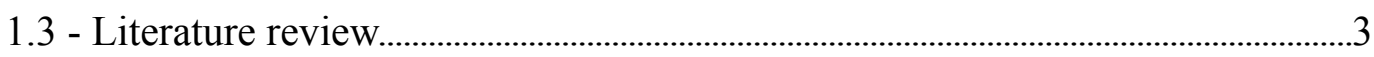

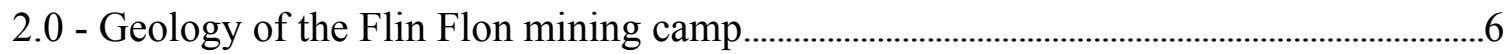

2.1 - TGI-3 Flin Flon.........................................................................................................

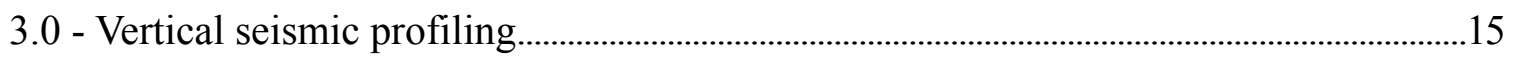

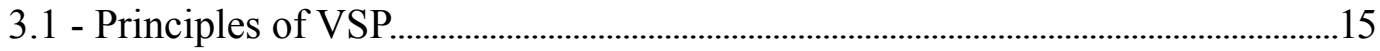

3.2 - Acquisition....................................................................................................

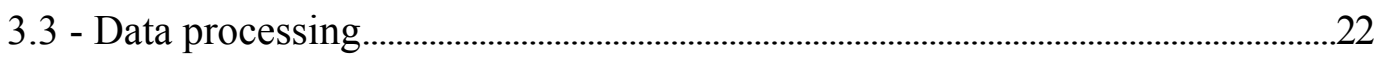

3.3.1 - Pre-processing ................................................................................................22

3.3.2 - Preliminary investigations using DSISoft..............................................23

3.3.3 - Data processing using Globe Claritas......................................................31 
3.4 - VSP observations.............................................................................................................52

4.0 - 3D finite difference modeling........................................................................................................63

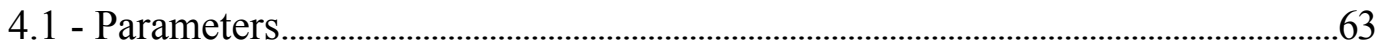

4.1.1 - Model assembly......................................................................................................63

4.1.2 - Pre-processing .................................................................................................

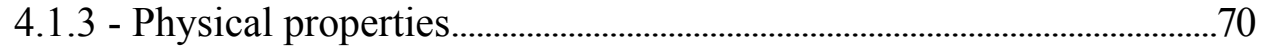

4.1.4 - 3D finite difference modeling using SOFI3D.........................................73

4.1.5 - Post-processing and outputs..........................................................................75

4.2 - 'Binary’ model observations.................................................................................................

4.3 - 'Ore/Missi' model observations.................................................................................105

4.4 - 'Lithological' model observations................................................................................107

4.5 - 'No Ore' model observations......................................................................................112

5.0 - Discussion and comparison of data sets..............................................................................115

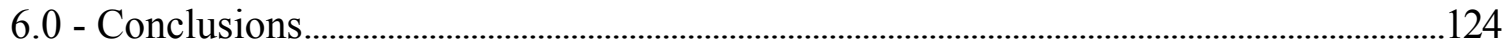

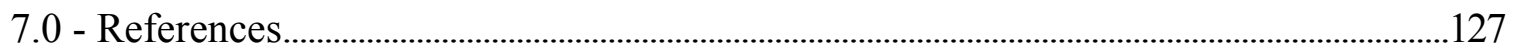




\section{List of tables}

Table 3.2.1 - Acquisition parameters..........................................................................................................20

Table 3.3.3.1 - Globe Claritas processing operations and parameters......................................................32

Table 3.4.1 - Summary of vertical component field VSP reflections..........................................................55

Table 3.4.2 - Summary of transverse component field VSP reflections...................................................58

Table 3.4.3 - Summary of radial component field VSP reflections......................................................61

Table 4.1.1.1 - Geological units represented in modeling voxets.............................................................65

Table 4.1.3.1 - Physical properties applied to voxets........................................................................

Table 4.1.4.1 - 3D finite difference modeling parameters......................................................................... 74

Table 4.2.1 - Summary of 'Binary’ model divergence reflections.............................................................86

Table 4.2.2 - Summary of 'Binary’ model rotation reflections....................................................................8

Table 4.2.3 - Summary of 'Binary’ model easting particle velocity reflections.......................................90

Table 4.2.4 - Summary of ‘Binary’ model northing particle velocity reflections....................................92

Table 4.2.5 - Summary of ‘Binary' model vertical particle velocity reflections........................................94

Table 4.2.6 - Summary of 'Binary (Callinan North)' model vertical particle velocity reflections.........96

Table 4.2.7 - Summary of 'Binary (Callinan East)' model vertical particle velocity reflections.............98

Table 4.2.8 - Summary of 'Binary (Callinan South)' model vertical particle velocity reflections........100

Table 4.2.9 - Summary of 'Binary (777)' model vertical particle velocity reflections...........................102

Table 4.3.1 - Summary of ‘Ore/Missi’ model vertical particle velocity reflections...............................107

Table 4.4.1 - Summary of 'Lithological' model vertical particle velocity reflections............................109

Table 4.5.1 - Summary of 'No ore' model vertical particle velocity reflections.......................................114 


\section{List of figures}

Figure 1.1.1 - Depiction of typical VSP survey geometry..........................................................................

Figure 2.1 - Flin Flon's location within the Trans-Hudson Orogen...........................................................6

Figure 2.2 - Geological map of the Flin Flon -Glennie Complex.............................................................

Figure 2.3 - Stratigraphic column of the Flin Flon-Callinan-777 mine area.............................................9

Figure 2.4 - Geological map of the Flin Flon study area.................................................................12

Figure 2.1.1 - The Flin Flon 3D Knowledge Cube..................................................................................... 14

Figure 3.1.1 - Cross section demonstrating the requirement for 3-component geophones......................16

Figure 3.1.2 - 3D path of borehole 4Q66W3 ..........................................................................................17

Figure 3.2.1 - Photo of VSP equipment at borehole 4Q66W3 .............................................................18

Figure 3.2.2 - Stacking chart for the first 8 shots of the 4Q66W3 VSP survey.........................................21

Figure 3.3.2.1 -AGC-scaled vertical component zero-offset dynamite field VSP data..........................24

Figure 3.3.2.2 - AGC-scaled horizontal component zero-offset dynamite field VSP data.....................26

Figure 3.3.2.3 - AGC-scaled vertical component zero-offset P-Vibroseis field VSP data.......................27

Figure 3.3.2.4 - AGC-scaled vertical component far-offset dynamite VSP data.....................................28

Figure 3.3.2.5 - Amplitude spectrum for VSP data shown in Figure 3.3.2.3 ............................................29

Figure 3.3.2.6 - Plan view depiction of far-offset VSP geometry for horizontal data rotation................31

Figure 3.3.3.1 - Raw vertical component zero-offset dynamite field VSP data..........................................34

Figure 3.3.3.2 - Field VSP data after spiking deconvolution.......................................................................36

Figure 3.3.3.3 - Amplitude spectrum of field VSP data after spiking deconvolution..............................37

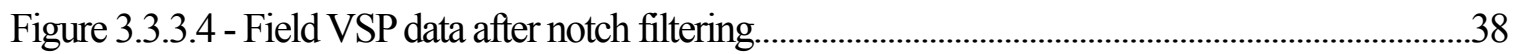


Figure 3.3.3.5 - Amplitude spectrum of field VSP data after notch filtering. .39

Figure 3.3.3.6 - Field VSP data after bandpass filtering....................................................................40

Figure 3.3.3.7 - Amplitude spectrum of field VSP data after bandpass filtering.......................................41

Figure 3.3.3.8 - $f-k$ spectrum of field VSP data after bandpass filtering ...................................................42

Figure 3.3.3.9 - Field VSP data after $f$ - $k$ filtering .............................................................................44

Figure 3.3.3.10 $-f-k$ spectrum of field VSP data after $f$ - $k$ filtering............................................................45

Figure 3.3.3.11 - Field VSP data after trace balancing.........................................................................46

Figure 3.3.3.12 - Field VSP data after FX deconvolution............................................................................48

Figure 3.3.3.13 - Final processed field VSP data...........................................................................49

Figure 3.3.3.14 - Amplitude spectrum of final processed field VSP data.................................................50

Figure 3.3.3.15 - $f-k$ spectrum of final processed field VSP data..............................................................51

Figure 3.4.1 - Annotated vertical component zero-offset dynamite field VSP data..................................54

Figure 3.4.2 - Annotated transverse component zero-offset dynamite field VSP data.............................57

Figure 3.4.3 - Annotated radial component zero-offset dynamite field VSP data....................................60

Figure 4.1.1.1 - Assembly of voxets for 3D finite difference modeling.....................................................66

Figure 4.1.1.2 - Ore zones of the 'Binary’ model....................................................................................67

Figure 4.1.1.3 - A new thrust fault built in gOcad....................................................................................68

Figure 4.1.5.1 - 'Lithological' model vertical particle velocity zero-offset VSP data..............................77

Figure 4.1.5.2 - 'Lithological' model 3D divergence wavefield visualization.........................................79

Figure 4.1.5.3 - 'Lithological' model 3D divergence wavefield visualization with acoustic

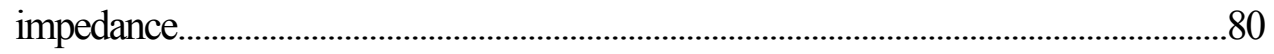

Figure 4.2.1 - 'Empty’ test model vertical particle velocity zero-offset VSP data......................................83 
Figure 4.2.2 - Annotated 'Binary’ model divergence zero-offset VSP data...............................................85

Figure 4.2.3 - Annotated 'Binary' model rotation zero-offset VSP data..................................................87

Figure 4.2.4 - Annotated 'Binary’ model easting particle velocity zero-offset VSP data.........................89

Figure 4.2.5 - Annotated 'Binary’ model northing particle velocity zero-offset VSP data.......................91

Figure 4.2.6 - Annotated 'Binary’ model vertical particle velocity zero-offset VSP data.........................93

Figure 4.2.7 - Annotated 'Binary (Callinan North)' model vertical particle velocity zero-offset VSP

data.

Figure 4.2.8 - Annotated 'Binary (Callinan East)' model vertical particle velocity zero-offset VSP

data

Figure 4.2.9 - Annotated 'Binary (Callinan South)' model vertical particle velocity zero-offset VSP

data. . .99

Figure 4.2.10 - Annotated 'Binary (777)' model vertical particle velocity zero-offset VSP data.........101

Figure 4.2.11 - 'Binary' model 3D divergence wavefield visualization with acoustic impedance......103

Figure 4.2.12 - 'Binary' model 3D rotation wavefield visualization with acoustic impedance...........104

Figure 4.3.1 - Annotated ‘Ore/Missi’ model vertical particle velocity zero-offset VSP data.................106

Figure 4.4.1 - Annotated 'Lithological' model vertical particle velocity zero-offset VSP data............108

Figure 4.4.2 - 'Lithological' model 3D divergence wavefield visualization with acoustic impedance. 110

Figure 4.4.3 - 'Lithological' model 3D rotation wavefield visualization with acoustic impedance....111

Figure 4.5.1 - Annotated 'No ore' model vertical particle velocity zero-offset VSP data. .113

Figure 5.1 - Comparing 'Binary', 'Binary (Callinan North)' and 'Binary (Callinan East)' data..........117

Figure 5.2 - Comparing 'Lithological', vertical and transverse component field data...........................122 


\section{List of appendices}

Appendix A - Processing operations and parameters for all field VSP data collected............................137

Appendix B - 3-component shot gathers for all field VSP data collected...............................................147

Appendix C - All synthetic VSP data from 3D finite difference modeling............................................171

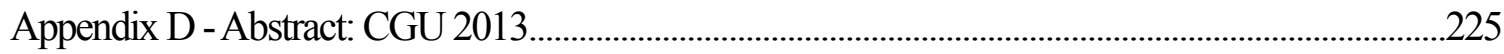

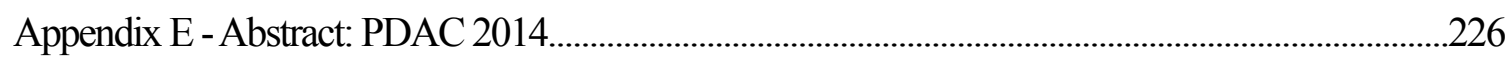

Appendix F - Abstract: AESRC 2014 ....................................................................................................22

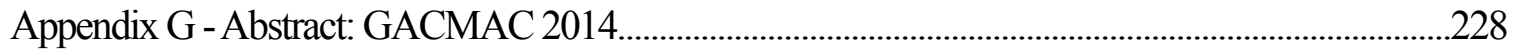

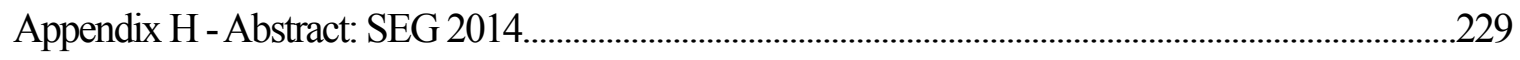




\section{0 - Introduction}

\section{1 - Background}

One of the main drivers of recent geophysical research in mineral exploration is the desire to investigate and define targets at depths beyond the limits of common airborne exploration methods. Seismic methods currently provide a means of achieving this goal. A vertical seismic profile (VSP) survey is a downhole seismic method by which geophones are deployed in a vertical or slightly deviated borehole and seismic sources are placed at various locations at the surface (Figure 1.1.1). Typically, VSP surveys are able to image a greater volume of rock around a borehole than downhole electromagnetic methods.

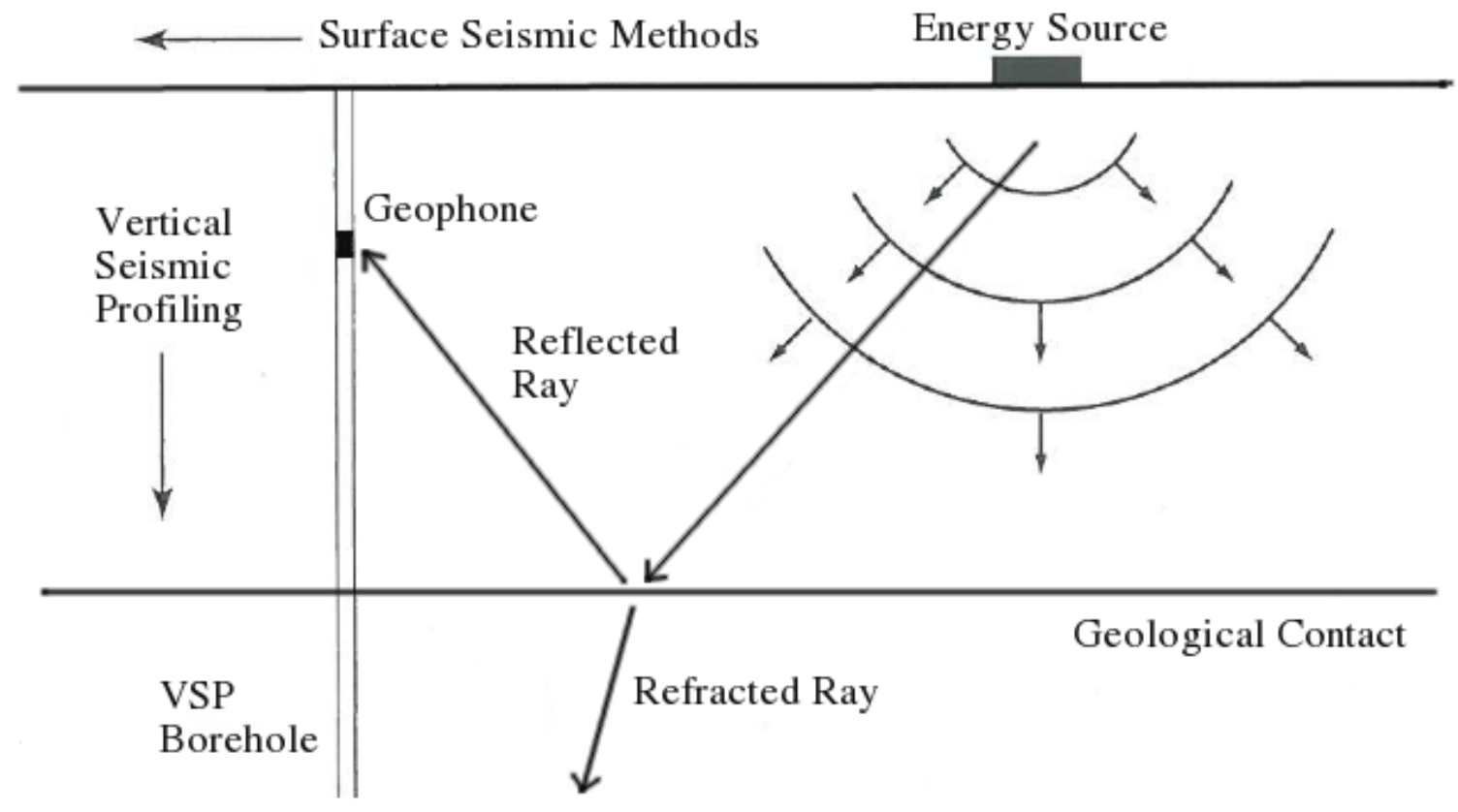

Figure 1.1.1 - Depiction of typical VSP survey geometry (modified after Hardage, 2000 and Hinds et al., 1996). 
In a VSP survey, geophones are located close to the subsurface region of interest. This offers the advantage of shorter seismic raypaths as compared to surface-based surveys. VSP surveying is commonly used in parallel with surface seismic methods in sedimentary basins for structural mapping and hydrocarbon reservoir characterization (Hardage, 2000). In contrast, VSP applications in crystalline settings have been limited due to the challenge of seismic imaging in environments with discontinuous reflectors.

\section{2 - Objectives and approach}

This study evaluates the potential of VSP surveying for mineral exploration. More specifically, the main objective is to process and model VSP data to detect and characterize the response of known volcanogenic massive sulphide (VMS) ore lenses. A second objective is to assess the performance of 3-component geophones in such surveys.

The approach chosen in this study is to compare a selection of field VSP data with synthetic analogues computed from geological models. First, field VSP data were acquired and processed to enhance as much of the upgoing wavefield as possible, potentially including a reflection signature of VMS ore lenses. A series of analogous 3D geological models were then built, from simple to more complex, in order to assess the contribution of different geological features to the observed seismic response. Through a 
comparison between the field and synthetic VSP shot gathers, reflections originating from the VMS ore lenses were identified and characterized.

The VMS mining camp in Flin Flon, Manitoba, Canada provides a suitable locality to test the proposed approach. For the Flin Flon camp, sufficient control on the geometry of the ore lenses and the host crystalline geology exists such that detailed 3D geological models have been previously built. Furthermore, several multi-offset VSP surveys have been conducted in deep boreholes in the camp. This study focuses on data from borehole 4Q66W3, chosen because its proximity and geometry relative to the known VMS lenses should allow a reflection signature of the VMS lenses to be captured in a VSP survey.

\section{3 - Literature review}

Downhole seismic techniques have been used as an exploration tool for almost a century. Interestingly, a 1917 patent by Reginald Fessenden describes the first use of a VSP survey geometry, which was intended to detect a subsurface ore body. The idea of deploying geophones in existing boreholes and using travel times from surface sources to delineate subsurface geological structure was further developed in McCollum and LaRue (1931). VSP surveys at that time were primarily focused on direct arrivals to determine wave propagation velocities near boreholes. It was not until the 1950s that seminal work by Jolly (1953), Riggs (1955) and Levin and Lynn (1958) demonstrated the importance of the seismic response following the direct arrivals (Hardage, 2000). 
VSP surveys, as with most seismic imaging methods, have seen the most use and development through structural applications in sedimentary environments by the oil and gas industry. Much of this was initiated by Soviet geophysicists from the 1950s through the 1970s, led by E.I. Gal'perin. Many books, workshops and papers were produced by this group showing the hydrocarbon applications of VSP (Gal'perin, 1974) and introducing the use of 3-component VSP records to determine the polarization of reflected seismic waves (Gal'perin, 1977). Kennett et al. (1980) provided an early discussion of the integration of VSP data with surface seismic data, as well as a comprehensive review of the processing and use of VSP data.

Electromagnetic, induced-polarization and gravity methods are typical methods employed for mineral exploration in crystalline environments but substantial effort has recently gone into developing seismic methods for this purpose. An important milestone in crystalline seismic imaging came with Green and Mair's study (1983), which demonstrated the imaging of complex fracture systems in a granitic body. Pretorius et al. (1989) followed this by successfully imaging ore-hosting structures in the Witwatersrand basin in South Africa and integrating the results with petrophysical data.

A Geophysical Developments volume entitled 'Hardrock Seismic Exploration' was released in 2003 by the Society of Exploration Geophysicists, documenting recent developments in acoustic property measurements (Salisbury et al., 2003), downhole seismic imaging (Perron et al., 2003) and 3D modeling (Bohlen et al., 2003) of metallic 
orebodies in crystalline environments. Considerable work has shown that practical imaging in such environments is not only possible with VSP surveying, but can be used to define geological structures and potentially lead to new discoveries (Miao et al., 1995, Eaton et al., 1996, Snyder et al., 2002 and Bellefleur et al., 2004).

The usefulness of creating synthetic seismic data to aid the interpretation of field data has long been known and synthetic VSP data have been compared to field VSP data since the early 1980s (e.g. Wyatt, 1981, Kelly et al., 1982 and Temme and Muller 1982). In the 1980s and 1990s, Computer-Aided Design methods were developed specifically for geological applications to visualize the subsurface (LaJaunie et al., 1997) and perform forward modeling (Hodgetts and Howell, 2000 and Malinowski et al., 2012). These tools have been continuously refined (e.g. deKemp, 2000, Caumon, 2004). This research project is a case study using many of these techniques, comparing field VSP data with 3D modeled synthetic analogues in a well-constrained VMS district to identify and characterize the response of known ore lenses. 


\section{0 - Geology of the Flin Flon mining camp}

The Flin Flon mining camp is located in northern Manitoba, Canada, within the Amisk collage of the 1.92-1.83 Ga Flin Flon-Glennie complex. The Amisk collage was accreted between the Archean Hearne, Sask and Superior cratons during the 1.84-1.80 Ga closure of the Manikewan Ocean and collisional stages of the Trans-Hudson orogeny. The TransHudson orogen was the largest Paleoproterozoic orogenic belt of Laurentia (Hoffman, 1988) and is host to many base metal sulphide occurrences.

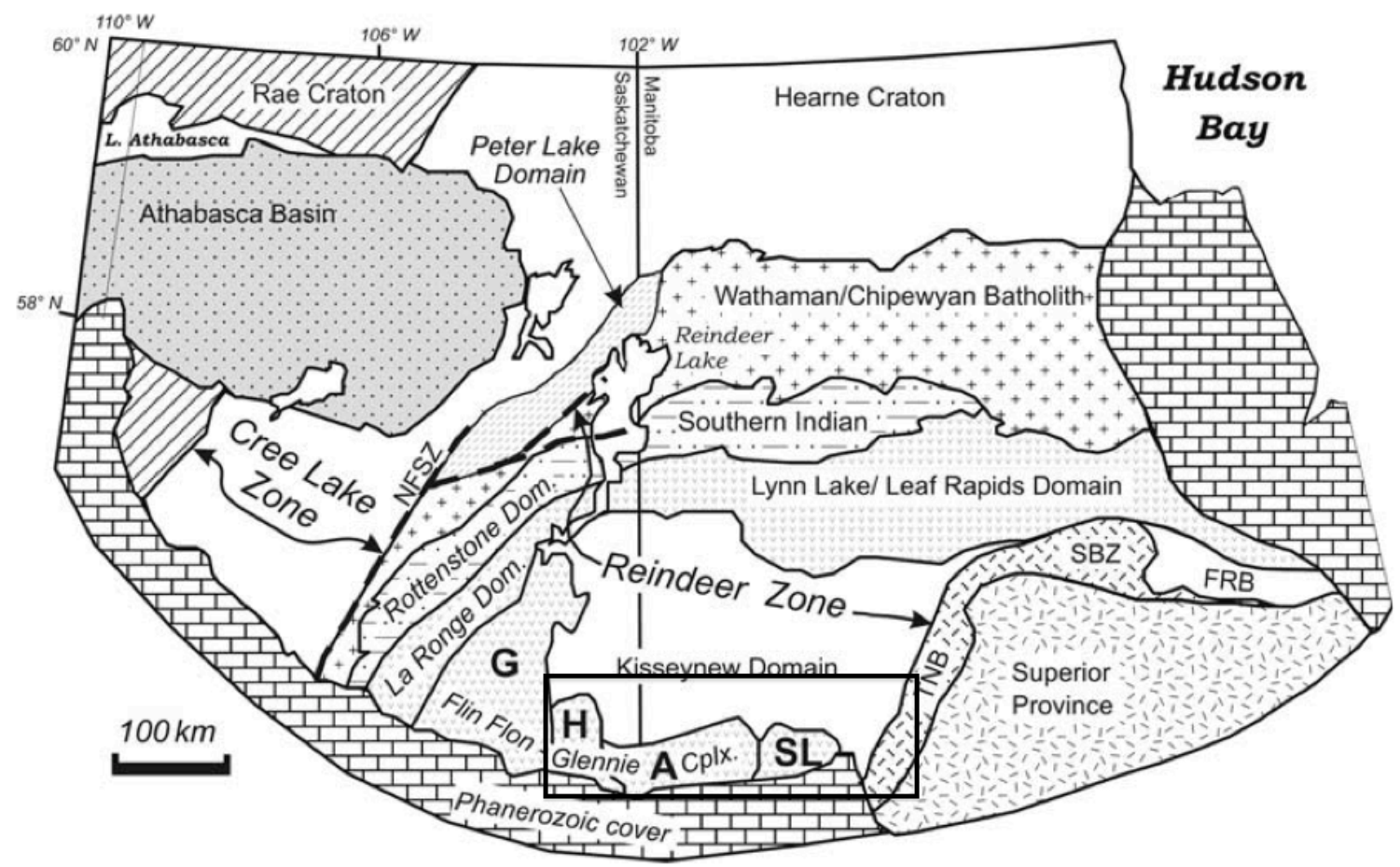

Figure 2.1 - Flin Flon's location within the Reindeer zone of the Trans-Hudson Orogen.

G: Glennie complex, H: Hanson Lake block. A: Amisk collage and SL: Snow Lake belt. The black box represents the area displayed in Figure 2.2 (modified after Corrigan et al., 2009). 
The Flin Flon-Glennie complex is one of several complexes within the THO, collectively referred to as the "Reindeer zone" (Lewry and Collerson, 1990), which were accreted along with the Sask craton between the much larger Hearne and Superior cratons (Figure 2.1). The Flin Flon-Glennie complex is made up of a group of volcanic arc, back-arc basin and ocean floor assemblages stitched by successor arc plutons and overlain by flysch and molasse basin sequences (Corrigan et al., 2009). The Amisk collage is structurally subdivided into fault-bounded blocks, overlain by the Missi group of fluvial and alluvial metasedimentary rocks (Figure 2.2), and characterized by sub-greenschist to greenschist metamorphic facies (Schetselaar et al., 2010).
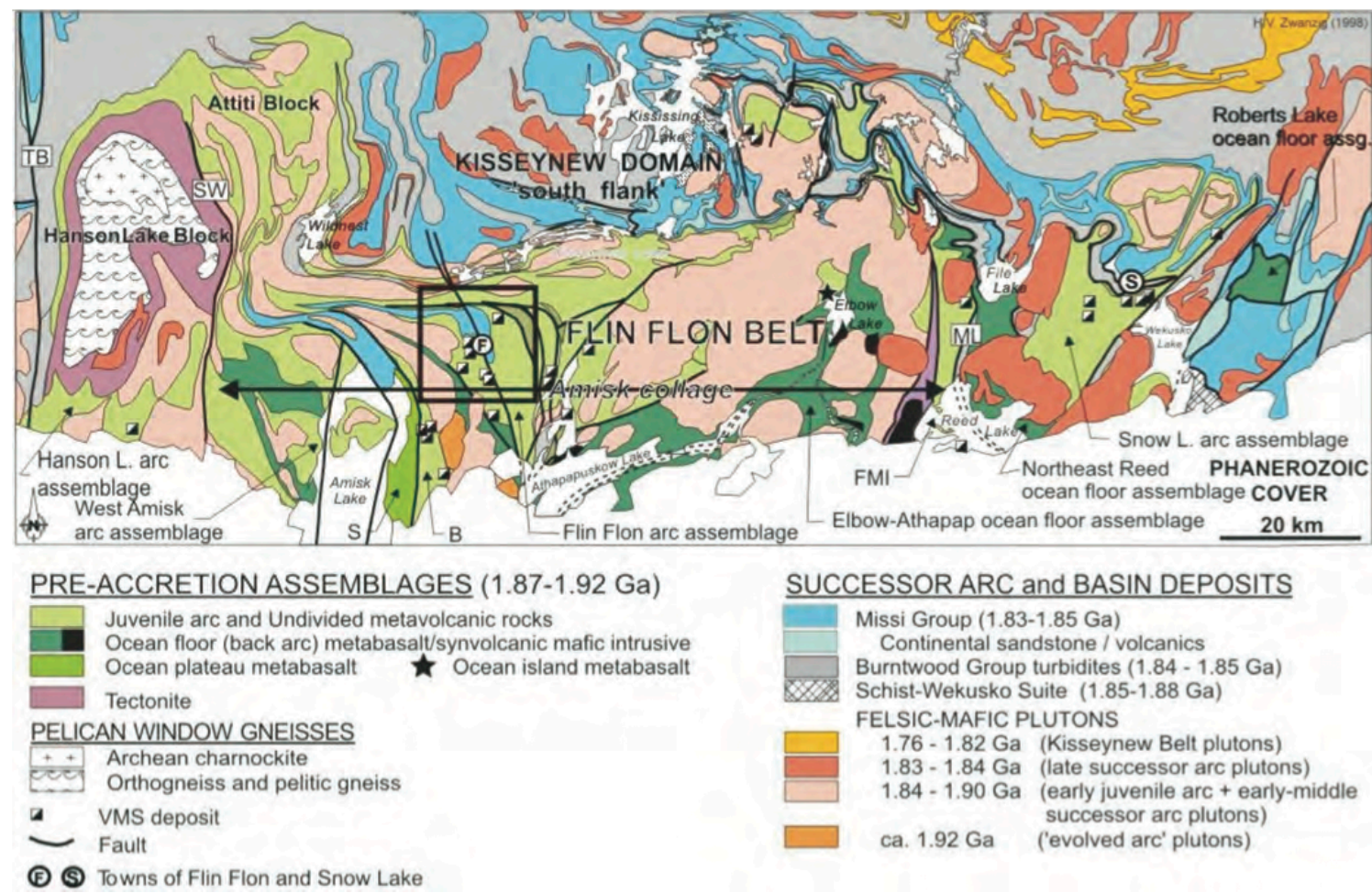

Figure 2.2 - Geology of the Flin Flon belt within the Flin Flon-Glennie Complex. The

black arrow shows the latitudinal extent of the Amisk collage and the black box

represents the area displayed in Figures 2.4 and 2.5 (modified after Galley et al., 2007). 
The stratigraphy is well-documented in the immediate vicinity of the Flin Flon main, Callinan and 777 VMS deposits (Figure 2.3) and is typical of a VMS-hosting submarine volcanic sequence. The footwall of the mine horizon is made up of the Blue Lagoon and Club members, each consisting mainly of mafic volcaniclastic rocks. The base of the Club member also contains a massive coherent rhyolite unit interpreted as a remnant of a rhyolite flow or dome (Devine, 2003; Gibson et al., 2005). Also present are rhyoliteclast-bearing mafic volcaniclastics, bedded mafic tuff and flows. Lying conformably above the Club member is the Blue Lagoon member, which includes pillow basalt flows intercalated between plagioclase-crystal-rich volcaniclastic rocks. Bedded tuff (i.e. millimeter - centimeter scale clasts) is present, but the member is dominantly a megabreccia (i.e. tens of meters scale clasts) and mafic in composition. Stratigraphic thickness of the unit is highly and abruptly variable, interpreted as flows and pyroclastic deposits infilling several synvolcanic fault-controlled sub-basins (Gibson et al., 2009). 


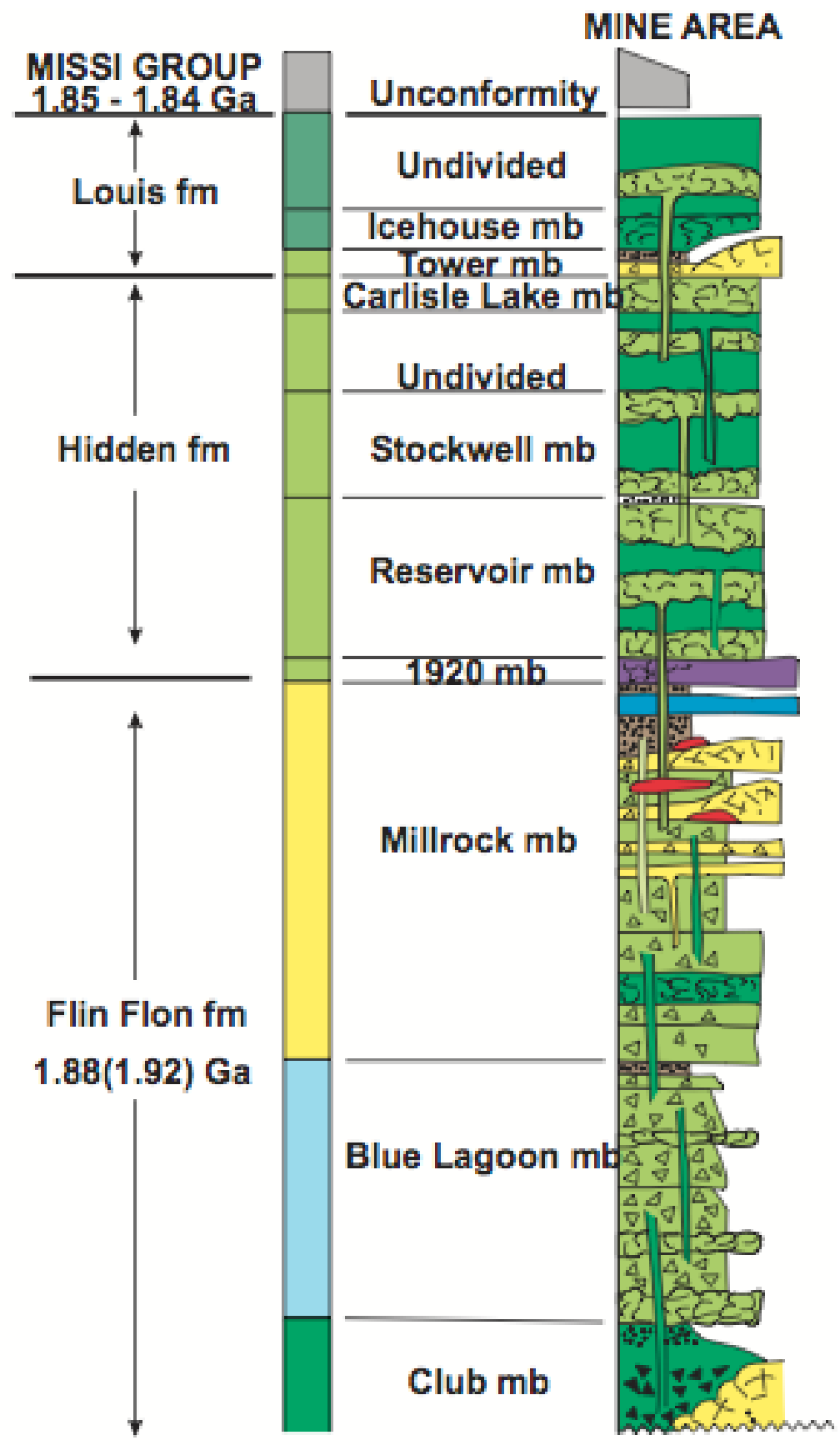

Figure 2.3 - Stratigraphic column of the Flin Flon-Callinan-777 mine area (Gibson et al., 2009). 
The Millrock member sits above the Blue Lagoon member and is host to the majority of known massive sulphide lenses in the camp. Again, much of the unit is composed of a similar mix of mafic megabreccia and basalt flows, but the upper Millrock member also includes several rhyolitic intervals. Rhyolite breccias with clasts up to 1 meter in size grade vertically and laterally into coherent massive rhyolite flows, interpreted as a dome / vent structure with associated flank breccias still present. Some of the mafic components are interpreted to represent submarine spatter and fire-fountain eruptions, in contrast to the pillowed flows seen below (Gibson et al., 2009). A laterally-continuous bedded tuff unit sits at the very top of the Millrock member and along with the underlying rhyolites represents the Flin Flon-Callinan-777 mine interval. Massive sulphide lenses of each deposit occur above, within and beside the rhyolites and underlie the bedded tuffs, indicating deposition in a hydrothermal system associated with the rhyolitic eruptions (Gibson et al., 2009). In some sections, eastward-dipping thrust repetitions are seen, which is consistent with regional structural trends. Again, lateral discontinuity of unit thicknesses indicates similar structural separation into sub-basins as seen in underlying units.

Immediately above the Millrock member sits the 1920 member of the Hidden formation, an Fe-Ti rich basalt / basaltic andesite flow. Above that, the Reservoir, Stockwell and Carlisle members of the Hidden formation are composed of massive mafic volcanic rocks with lesser pillowed flows and mafic volcaniclastic rocks. Much of the mafic volcanics include tuff fragments or are peperitic, indicating flow emplacement on or within a loose 
submarine surface. Conformably overlying the Hidden formation are the Tower member rhyolites and bedded tuffs of the Louis formation, representing a later, shorter interval of felsic volcanism similar to that of the Millrock member. The Icehouse and other undivided members of the Louis formation are very similar mafic flows and minor volcaniclastics to those of the Hidden formation.

The Flin Flon arc assemblage was overprinted by several intrusive suites, including the 1.87 - $1.85 \mathrm{Ga}$ Annabel and Channing granitoids, the $1.84 \mathrm{Ga}$ mafic to ultramafic Boundary intrusions and the 1.84 Ga bimodal Phantom intrusions (Stern et al., 1999). A major unconformity is locally represented by the contact between the arc volcanics and successor plutonics and the base of the Missi clastic metasedimentary basin which overlies much of the region. The Missi group is made up of foliated, weakly bedded arkose and greywacke with minor pebble interbeds and conglomerate (Syme and Bailes, 1993). 


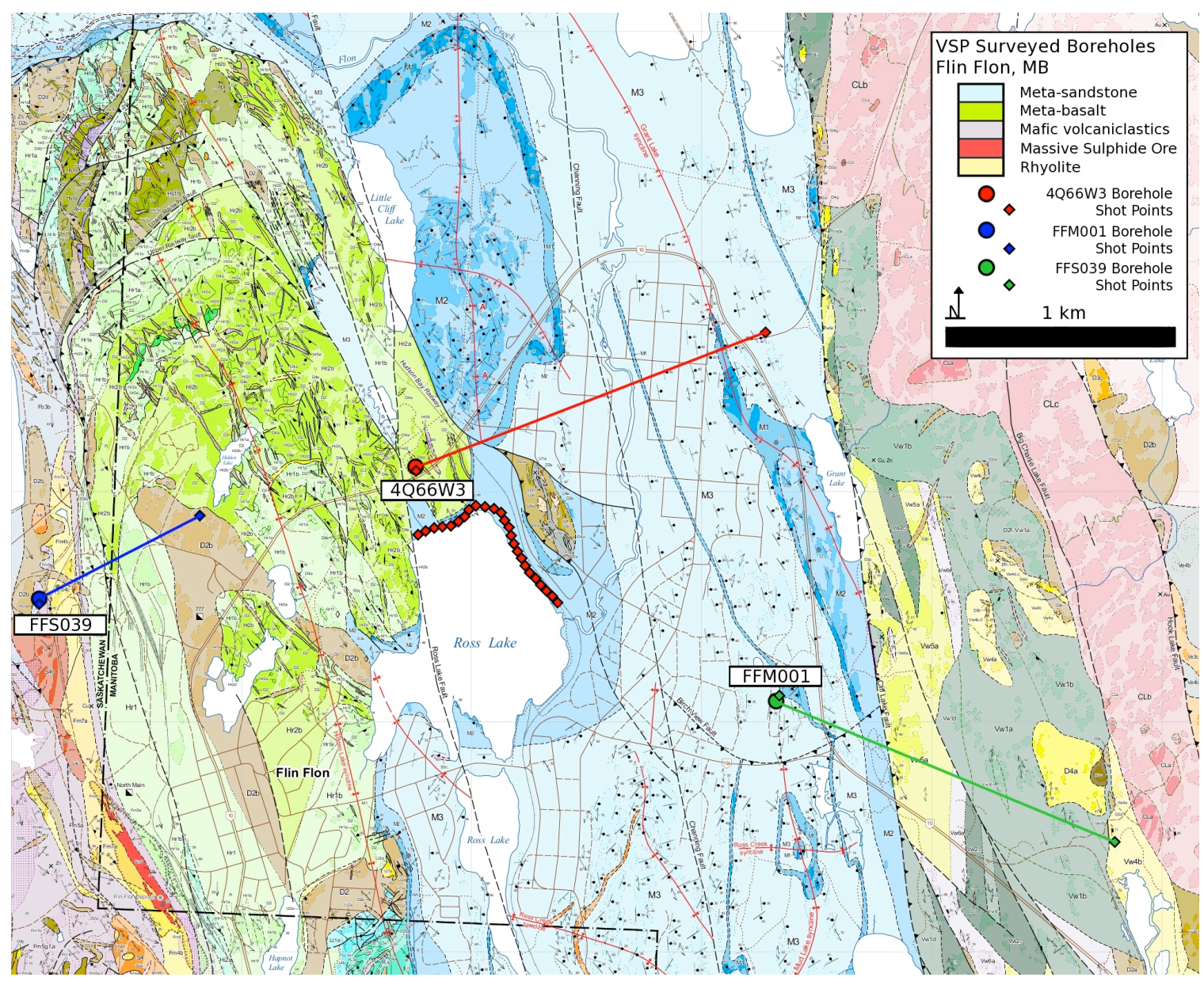

Figure 2.4 - Geological map of the town of Flin Flon with VSP-surveyed boreholes and source locations used in this study. The lower left corner of the map area is located at $313990 \mathrm{~m}$ [E] and $6071910 \mathrm{~m}$ [N], UTM, NAD83 Zone 14 (modified after Simard et al., 2010).

Seven ductile deformational events are recorded by rocks of the Flin Flon assemblage.

$\mathrm{D}_{1}$ and $\mathrm{D} 2$ were folding events resulting from the intraoceanic accretion of several volcanic arc terranes, best represented by the D1 Burley Lake syncline and D2 Hidden Lake syncline. Prominent eastward-dipping thrust stacks and west-verging folds were formed by the D3 fold-thrust system interpreted as representing the accretion of the Flin 
Flon and Glennie terranes. D4 thrusting then formed southward-dipping thrust stacks, possibly due to the collision of the complex with the Sask craton. D5 and D6 both formed regionally penetrative cleavages and $\mathrm{D} 7$ reactivated thrust faults, all related to large-scale intercratonic collisions in the terminal closure of the Manikewan ocean.

The Flin Flon belt is richly endowed with VMS deposits including the 85.5 Mt Flin FlonCallinan-777 Cu-Zn-Au-Ag ore system. Chalcopyrite, pyrrhotite and sphalerite-rich ore lenses are hosted in altered rhyolites of the Millrock member (Schetselaar et al., 2010). At least three thrust-stacked intervals of the Millrock member contain VMS ore lenses with varying grades, textures and compositions of ore. Lateral and vertical zonation of $\mathrm{Cu}$ and $\mathrm{Zn}$ rich minerals within individual lenses has been documented (Byron et al., 2010) and ore textures range from massive lenses to stockwork veins and disseminated sulfides in the altered rhyolites. Intense chlorite-carbonate and clay alteration haloes exist around the lenses, evidence of the extent of the hydrothermal system which produced and enriched the VMS ore (White et al., in press). Also, many lenses of the Callinan deposit were mined out prior to the field VSP survey and backfilled with crushed waste rock in either an unconsolidated or "cemented" form (White et al., 2012).

\section{1 - TGI-3 Flin Flon}

The Flin Flon mining camp has been extensively mapped, drilled, mined and targeted by geophysical surveys for decades, leading to a wealth of geoscience data, interpretations 
and 3D geological models. Recently, the area was a focal point for multidisciplinary research as part of the Targeted Geoscience Initiative-3 (TGI-3) program by the Geological Survey of Canada (GSC), Hudbay Minerals Inc. and several universities. The VSP data used in this study were an output of this project, as well as a 3D seismic volume, new geological maps and a 3D geological model. This $7.5 \mathrm{~km}$ (northing) by 5 km (easting) by $2 \mathrm{~km}$ (depth) model, known as the Flin Flon 3D Knowledge Cube, was assembled from a drill hole database, mine plans, 3D ore models, 2D seismic sections, a 3D seismic cube and geological maps provided by the GSC and Hudbay Minerals Inc (Figure 2.1.1, Schetselaar et al., 2010).

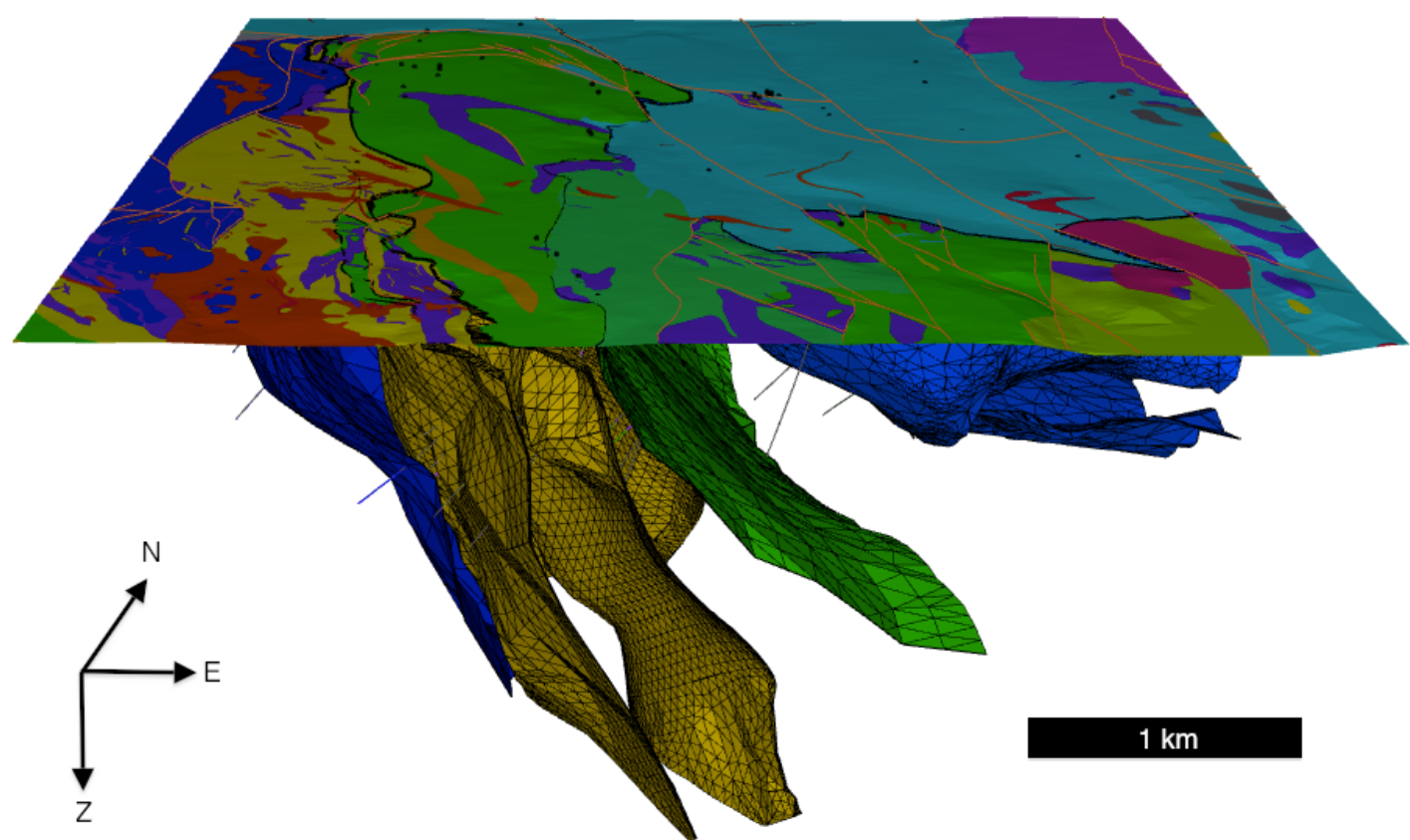

Figure 2.1.1 - Oblique view of the Flin Flon 3D Knowledge Cube, a 3D geological model built for the Flin Flon TGI-3 used as the basis for all models built in this study. Major colours correspond to geological units and rock types shown in Figures 2.3 and 2.4 (modified after Schetselaar et al., 2010). 


\section{0 - Vertical Seismic Profiling}

\section{1 - Principles of VSP}

Conventional zero-offset VSP survey geometry describes a vertical borehole and receiver array with sources placed as close to the borehole collar as possible and reflectors parallel to the surface. This geometry is ideal because the reflectors are perpendicular to the direct wave propagation and to the receiver array, and therefore the orientations of particle motion of the direct and reflected P- and S-waves are largely separated on the recorded vertical (P-wave) and horizontal (S-wave) components. By varying the distance and azimuth, or offset, of the source relative to the borehole containing the receiver array, the orientations of particle motion of waves and the volume of material imaged is affected. Increasing the offset of the source from the receiver array and the targeted reflectors also decreases the signal-to-noise ratio, which places a limitation on the survey extent. The survey geometry is extremely important for the processing and interpretation of VSP data.

In deviated boreholes the geophone components are sequentially rotated as the axis of the borehole changes direction. Also, in environments with dipping reflectors the orientations of particle motion of reflected P- and S-waves will be different than those of the associated direct waves. In these VSP surveys 3-component geophones are required to accurately capture the particle motion, as demonstrated in Figure 3.1.1. The use of 3- 
component geophones and at least one offset source may also be used to indicate the direction to and geometry of the targeted reflectors. Deviated boreholes were used for this survey (3D geometry of 4Q66W3 is shown in Figure 3.1.2).

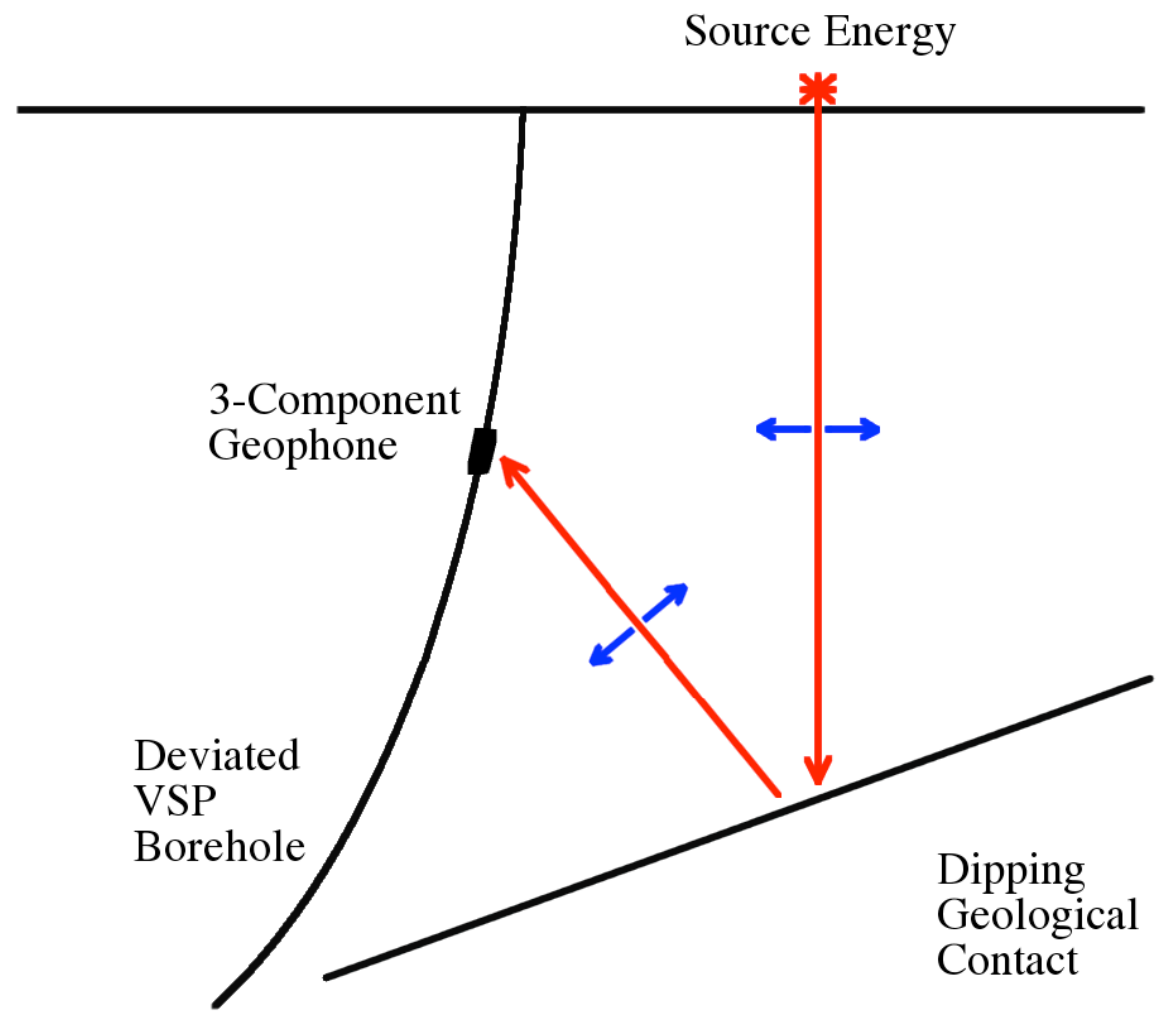

Figure 3.1.1 - Cross-section demonstrating the theoretical requirement for 3-component geophones in VSP surveys with dipping reflectors and deviated boreholes.. Red arrows illustrate the propagation direction and parallel particle motion of direct and reflected Pwaves and blue arrows illustrate the orientations of particle motion for direct and reflected S-waves. In this case, there is an almost $45^{\circ}$ difference in the propagation direction and orientations of particle motion between the direct and reflected waves due to the geometry of the reflector. This and the rotation of the geophone caused by the deviated borehole require a 3-component geophone to be used to accurately record the particle motion. 


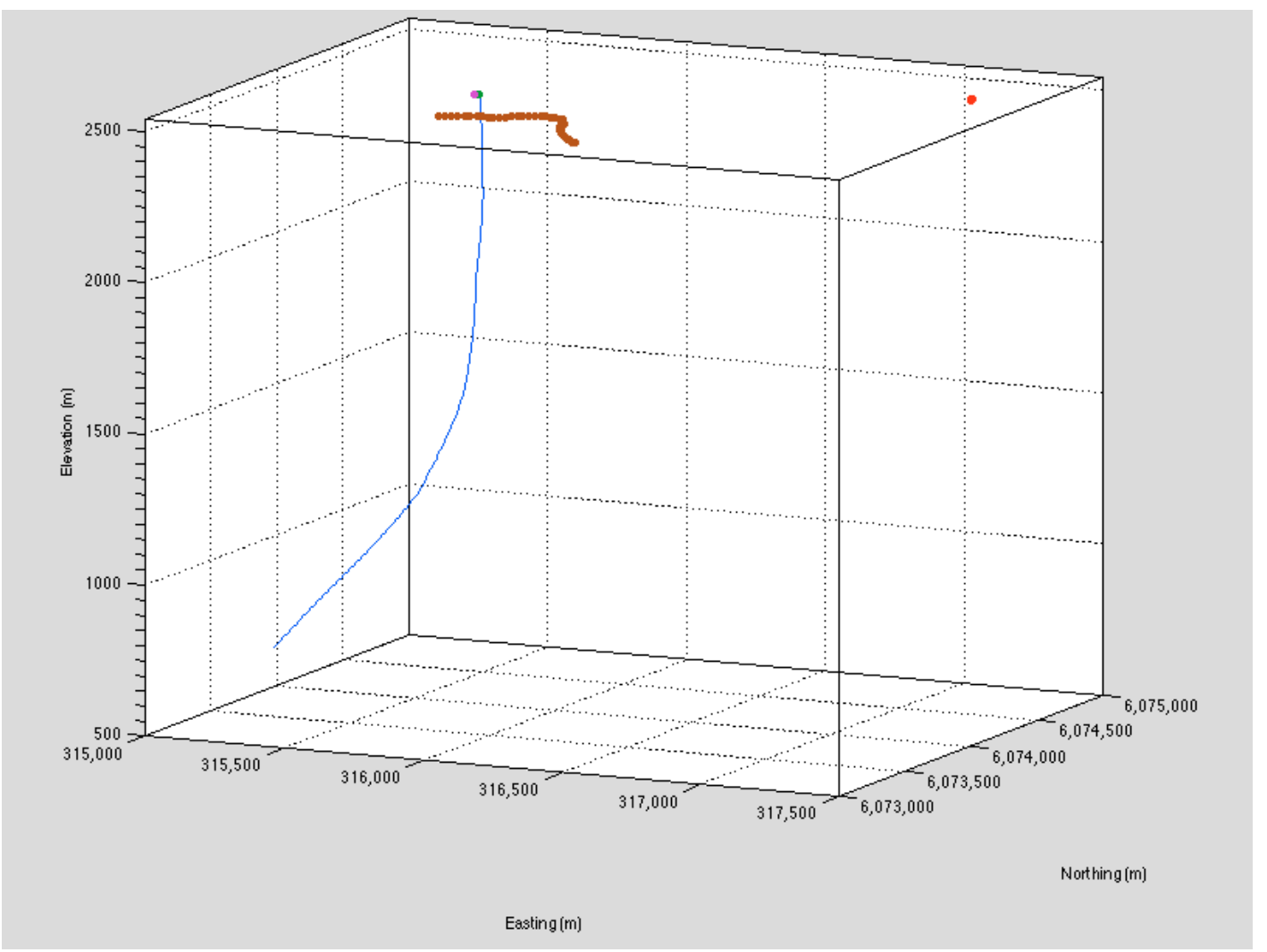

Figure 3.1.2 - 3D path of the deviated borehole 4Q66W3 used in the Flin Flon VSP

survey. The blue line represents the well while surface points represent the zero-offset (green and pink), far-offset (red) and walkaway (burgundy) source locations.

Coordinates are in UTM meters, NAD83 Zone 14.

The contrast in acoustic impedance between two geological units is the physical boundary which will reflect seismic waves. The proximity of the reflector to the borehole will also affect whether or not an upgoing reflection is captured by the receiver array as waves attenuate through dense geological media. Strong reflections far from a borehole and weaker reflections near the borehole may produce similar amplitudes in a VSP record, but the timing and apparent velocities of the two signals could be used to distinguish them. Apparent velocity is used to describe the measured velocity along the 
axis of the receiver array of a reflected wave originating at an off-hole reflector. Since the propagation direction of a reflected wave could be perpendicular to the receiver array, apparent velocities may be much faster than the velocity of the material through which the wave is propagating.

\section{2 - Acquisition}

Between October 12th and 18th, 2006, VSP data were acquired from 3 wells in Flin Flon: 4Q66W3, FFM001 and FFS039 (see Figure 2.4 for locations). After the equipment was received in Flin Flon, a similar procedure for each of the three wells was followed as described below:

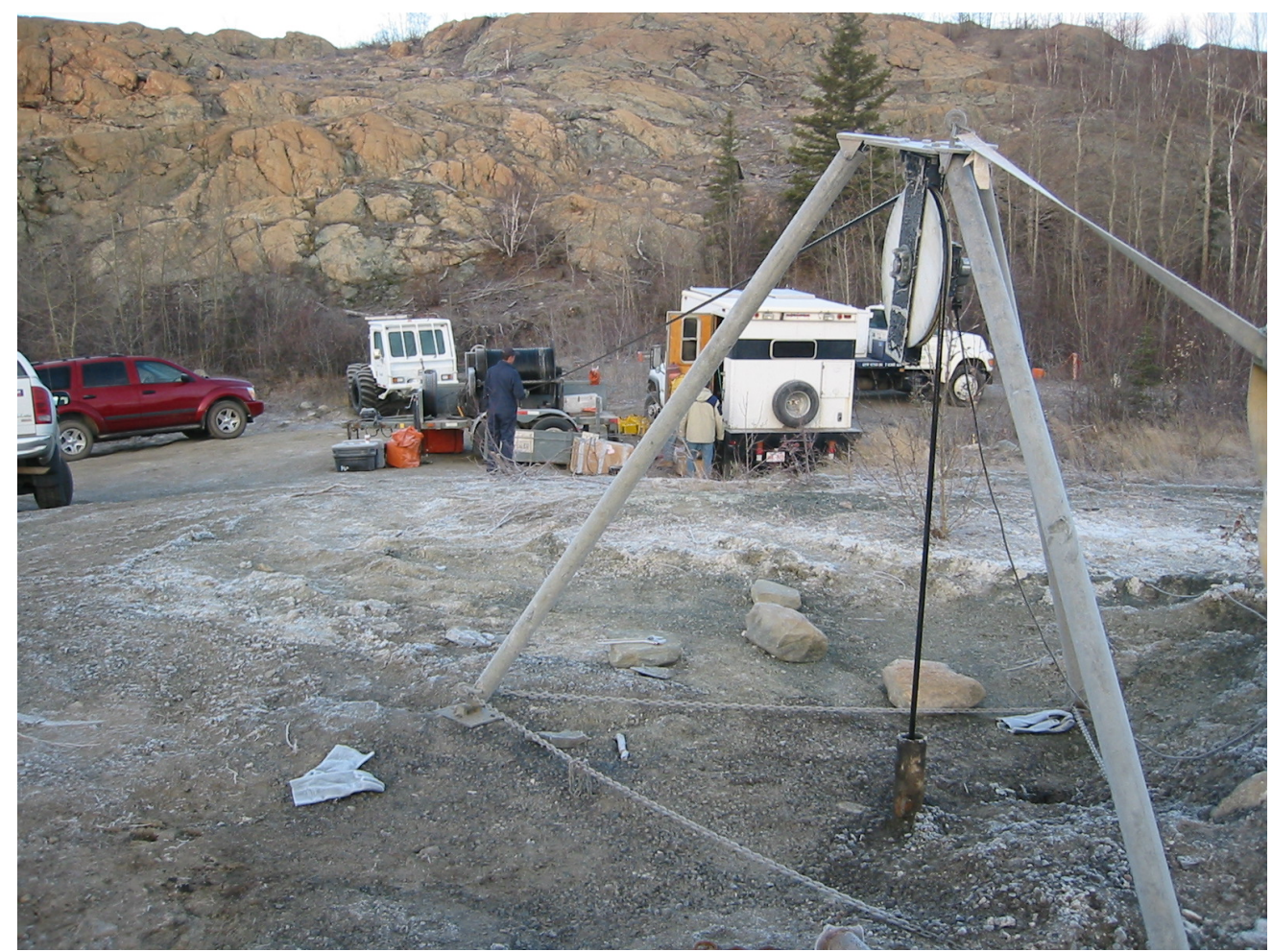

Figure 3.2.1 - VSP equipment assembled and deployed in borehole 4Q66W3 (photo by

G. Bellefleur). 
1) All equipment was transported to the well collar. This included the Vibrometric 8level 3-component geophone tool, control boxes, tripod assembly, winch and $2.2 \mathrm{~km}$ cable spool, a 240-channel Geometrics recording system and surface geophone array, 2 mini-Vibroseis sources (a truck-mounted $6000 \mathrm{lb}$ source for P waves and a buggymounted $12000 \mathrm{lb}$ source for $\mathrm{S}$ waves) and dynamite source gear (Table 3.2.1).

2) The survey equipment was assembled, tested and deployed. The geophone tool, triggers for the mini-Vibroseis and dynamite sources, control boxes and recording truck were connected. The geophone tool was initially lowered to the maximum depth and the clamping arms deployed to firmly couple the geophone tool against the well casing.

3) The geophone tool, sources and recording system were armed.

4) Each source was triggered and data was recorded. The source order was zero-offset dynamite, far-offset dynamite ( $3 \mathrm{~s}$ recording at $0.25 \mathrm{~ms}$ sample rate), $\mathrm{P}$-wave Vibroseis and S-wave Vibroseis, (8 s sweep and correlation, $12 \mathrm{~s}$ recording at $1 \mathrm{~ms}$ sample rate). Data was captured digitally as SEG-2 format dat files with each component of the tool and surface array separated (parameters of the survey acquisition are shown in Table 3.2.1). Dynamite shots were preloaded in $\sim 5 \mathrm{~m}$ deep shot holes drilled into bedrock prior to the survey by Cambrian Blasting. 
5) The geophone tool was unclamped and raised in alternating $5 \mathrm{~m}$ and $75 \mathrm{~m}$ increments between each set of shots (zero-offset dynamite, far-offset dynamite, P Vibroseis, S Vibroseis). Each level of the geophone tool is spaced $10 \mathrm{~m}$ apart achieving a single fold survey with a $5 \mathrm{~m}$ sample interval (stacking chart for 4Q66W3 shown in Figure 3.2.2).

Table 3.2.1 - Acquisition parameters for the Flin Flon VSP survey.

\begin{tabular}{|l|l|l|l|}
\hline Surveyor & \multicolumn{3}{|l|}{ Geological Survey of Canada (GSC) } \\
\hline Source types & Dynamite & P Vibroseis & S Vibroseis \\
\hline Source Contractor & Cambrian Blasting & University of Alberta & GSC \\
\hline Sources & $\begin{array}{l}\text { pentolite, } \\
\text { various-sized charges }\end{array}$ & $\begin{array}{l}\text { IVI Truck-mounted, } \\
6000 \mathrm{lb}\end{array}$ & $\begin{array}{l}\text { IVI Minivib, } \\
12000 \mathrm{lb}\end{array}$ \\
\hline Sweep Length (Vibroseis) & $8 \mathrm{~s}$ \\
\hline Sweep Frequency (Vibroseis) & $5-250 \mathrm{~Hz}$ \\
\hline Geophone Tool & Vibrometric R8-XYZ \\
\hline Levels & 8 \\
\hline Components & 3 Orthogonal \\
\hline Level Interval & $10 \mathrm{~m}$ \\
\hline Shot Stacking Pattern & $5 \mathrm{~m}, 75 \mathrm{~m}$ \\
\hline Sampling Interval & $5 \mathrm{~m}$ \\
\hline Recording Contractor & University of Alberta \\
\hline Recording Length & $3 \mathrm{~s} \mathrm{(Dynamite),} \mathrm{12} \mathrm{s}$ (Vibroseis) \\
\hline Sample Rate & 0.25 ms (Dynamite), $1 \mathrm{~ms}$ (Vibroseis) \\
\hline
\end{tabular}

\begin{tabular}{|l|c|c|c|}
\hline Borehole & 4Q66W3 & FFM001 & FFS039 \\
\hline Wireline Depth Interval & $161.5-1116.5 \mathrm{~m}$ & $330-1770 \mathrm{~m}$ & $230-1105 \mathrm{~m}$ \\
\hline Date & $12-$ Oct-06 & 16-Oct-06 & 18 -Oct-06 \\
\hline Zero-Offset Dynamite & $\checkmark$ & $\checkmark$ & $\checkmark$ \\
Zero-Offset P Vibroseis & $\checkmark$ & & \\
Zero-Offset S Vibroseis & $\checkmark$ & $\checkmark$ & $\checkmark$ \\
Far-Offset Dynamite & $\checkmark$ & & \\
Walkaway P Vibroseis & $\checkmark$ & & \\
\hline
\end{tabular}




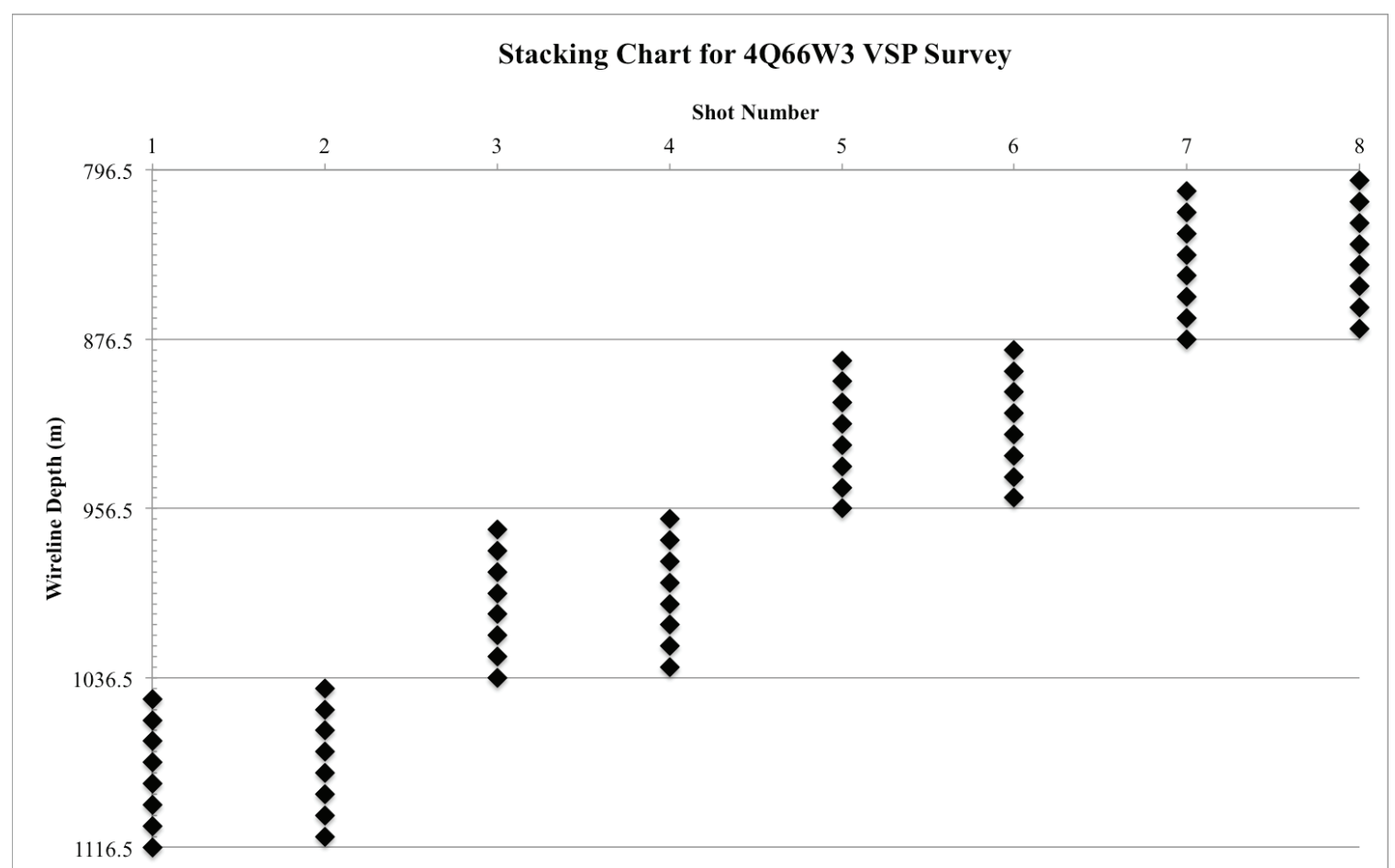

Figure 3.2.2 - Stacking chart for the first 8 shots of the 4Q66W3 VSP survey. The geophone tool with $10 \mathrm{~m}$ spacing was raised between each set of shots (zero-offset dynamite, far-offset dynamite, P Vibroseis, S Vibroseis) in alternating 5 and $75 \mathrm{~m}$ increments to acquire a single-fold survey with a $5 \mathrm{~m}$ sample interval.

An important consideration in the design of a seismic survey is the spatial and temporal resolution of the data. Based on the velocities, frequency range and raypaths between the source, reflectors and receivers, a minimum detectable reflector radius known as the Fresnel zone can be determined. Using the method described in Hardage, 2000 and the parameters of this VSP survey, a first-order Fresnel zone with a radius between 112 and $227 \mathrm{~m}$ is defined. For comparison, the targeted ore lenses in this study are between $\sim 100$ and $1200 \mathrm{~m}$ in length and $\sim 30-500 \mathrm{~m}$ in width. 


\section{3 - Data processing}

\subsection{1 - Pre-processing}

Once field collection of all VSP data in Flin Flon was completed, the raw SEG-2 data were pre-processed and sorted into shot gathers and rewritten to standard SEG-Y Rev 1.0 format. All data were written as integers in four-bit native floating point format using MATLAB. The reordering of traces was particularly challenging due to the number and locations of sources, the 3-component nature of the data and the necessity of interleaving traces to achieve a $5 \mathrm{~m}$ sample interval.

Zero-offset Vibroseis-source data were cross-correlated to produce impulse-equivalent data. An assessment of the walk-away Vibroseis data revealed very low signal-to-noise levels and the data were not considered further in this study (Schmitt, 2006). Additional information including source types and locations, sample rates, data ordering and receiver locations were stored in the trace headers in each SEG-Y file. All VSP data and accompanying documentation were then written to DVDs and shipped to the Earth Imaging and 3D Modeling group at the GSC for further work. 


\subsection{2 - Preliminary investigations using DSISoft}

Upon receipt of the data at the GSC, the zero-offset dynamite data was processed preliminarily for integration with the simultaneously-acquired 3D seismic volume, comparison with downhole geological and geophysical logs and to assess the results of source tests. This study focused on reflectors which intersect the borehole and could be traced laterally into the 3D seismic volume for interpretative purposes. In general, correlations between strong reflections and some of the primary lithological boundaries obtained from drill cores were found (Dieteker et al., 2007).

For this study, initial investigation and preliminary data processing was accomplished using the public domain DSISoft suite of VSP processing tools in MATLAB, developed by the Downhole Seismic Imaging Consortium and the GSC (Beaty, 2002). The software suite contains data conversion algorithms, signal processing modules, visualization, graphical analysis and image writing tools. Since the suite is written entirely as MATLAB scripts, all modules are customizable and new tools in the same style can easily be added by the user. During processing, the .mat files can be viewed as databases to monitor the effects of individual operations. In this way, DSISoft serves as an excellent platform to actively teach the fundamentals of VSP processing to a new user and a preliminary processing sequence using DSISoft was developed for the Flin Flon data. 
The first task was to assess the quality of each data set, identify trends or variability and to decide which data would be most useful for this study (Figures 3.3.2.1 shows an example of raw VSP data). All SEG-Y data were imported into DSISoft, separated into vertical and horizontal components and displayed graphically with automatic gain control (AGC) scaling as black wiggle traces with variable area. It should be noted that since borehole 4Q66W3 is deviated, the geophone components are not consistently vertical and horizontal.

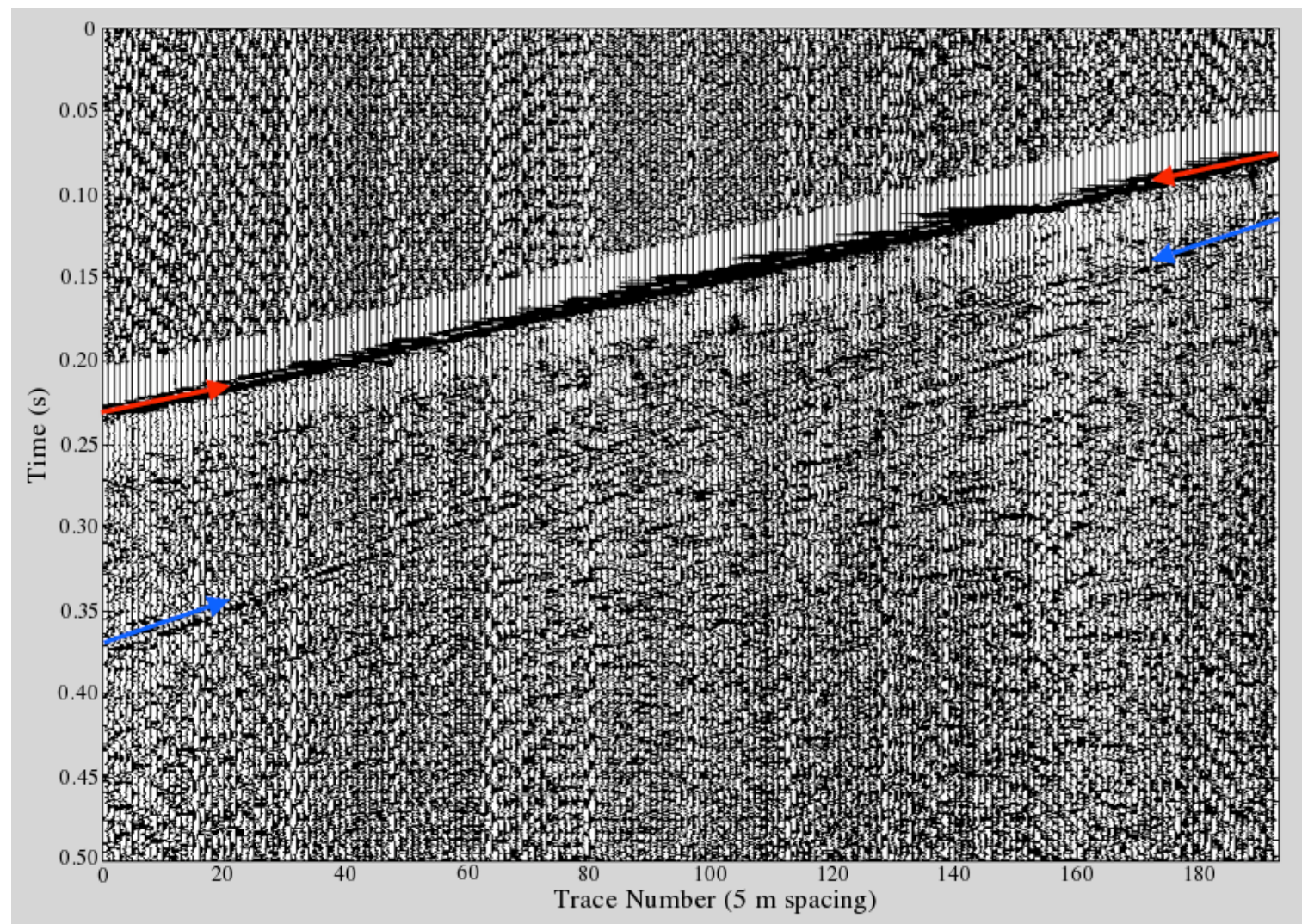

Figure 3.3.2.1 - AGC-scaled vertical component zero-offset dynamite data for 4Q66W3.

Downgoing P- and S-waves are clearly seen, annotated with red and blue arrows respectively. Note the low-amplitude window around the downgoing P-wave; an artifact of AGC scaling. 
Although there is strong overprinting of noise, the downgoing P-waves in the vertical component data and downgoing S-waves in the horizontal component data could be consistently identified (Figure 3.3.2.2). At first glance, both zero and far-offset dynamite data appeared to show more detail than any of the Vibroseis data sets (Figure 3.3.2.3). Any energy observed on traces before the first arrival of the downgoing P-wave must be noise since the source energy has not yet arrived at each geophone. As expected, the downgoing signal in the far-offset data set is significantly weaker but can still be observed (Figure 3.3.2.4). The strong electrical noise ( $60 \mathrm{~Hz}$ and several harmonics) occurs throughout each of the data sets and is clearly identified in amplitude spectra (Figure 3.3.2.5). Tube waves, recognized by their anomalously low velocity, were also investigated and not identified in any of these data sets. 


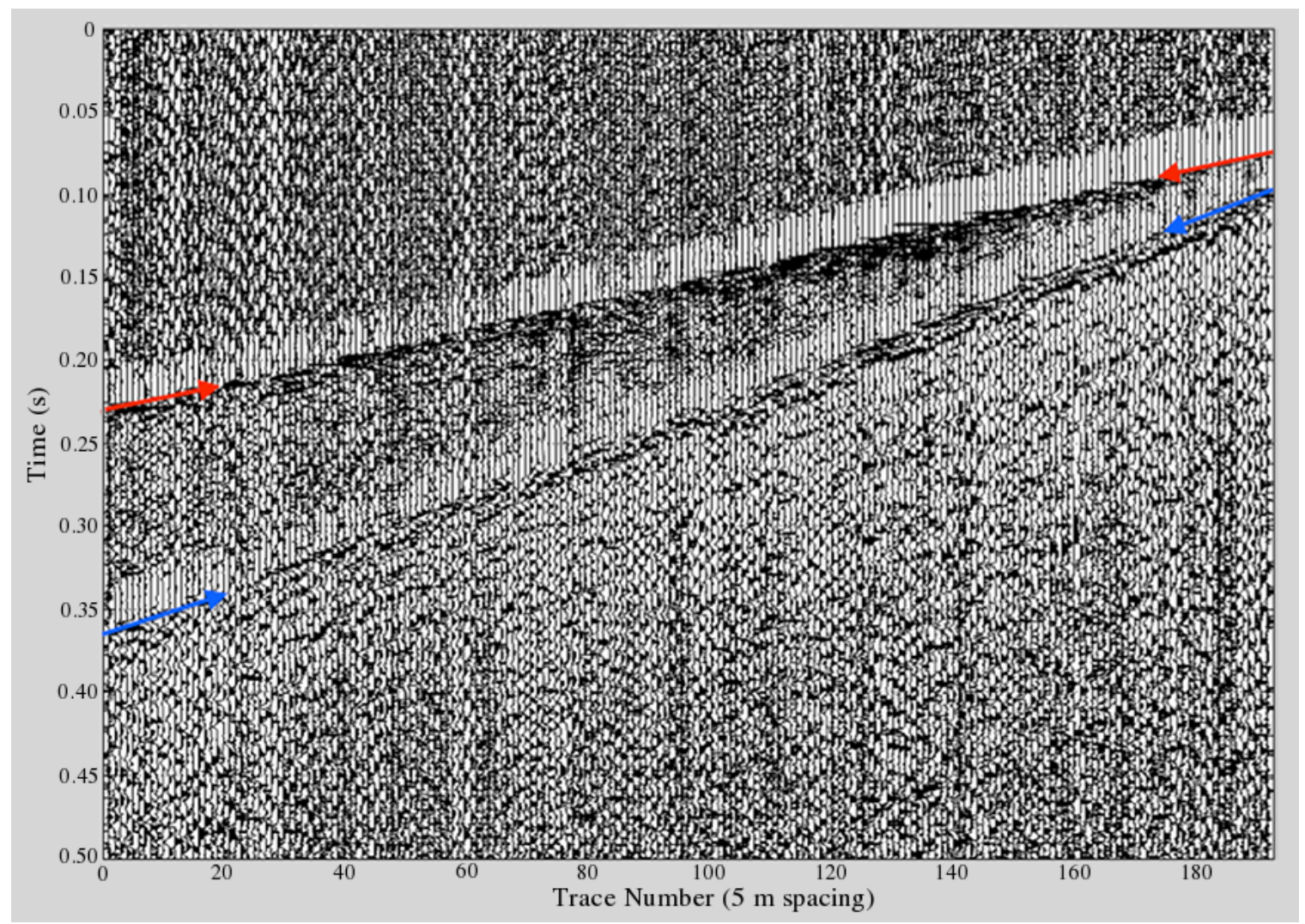

Figure 3.3.2.2 - AGC-scaled horizontal component zero-offset dynamite data for

4Q66W3. Downgoing P- and S-waves are clearly seen, annotated with red and blue arrows respectively. Note that the downgoing S-wave is stronger here than on the corresponding vertical component section (cf. Figure 3.3.2.1). 


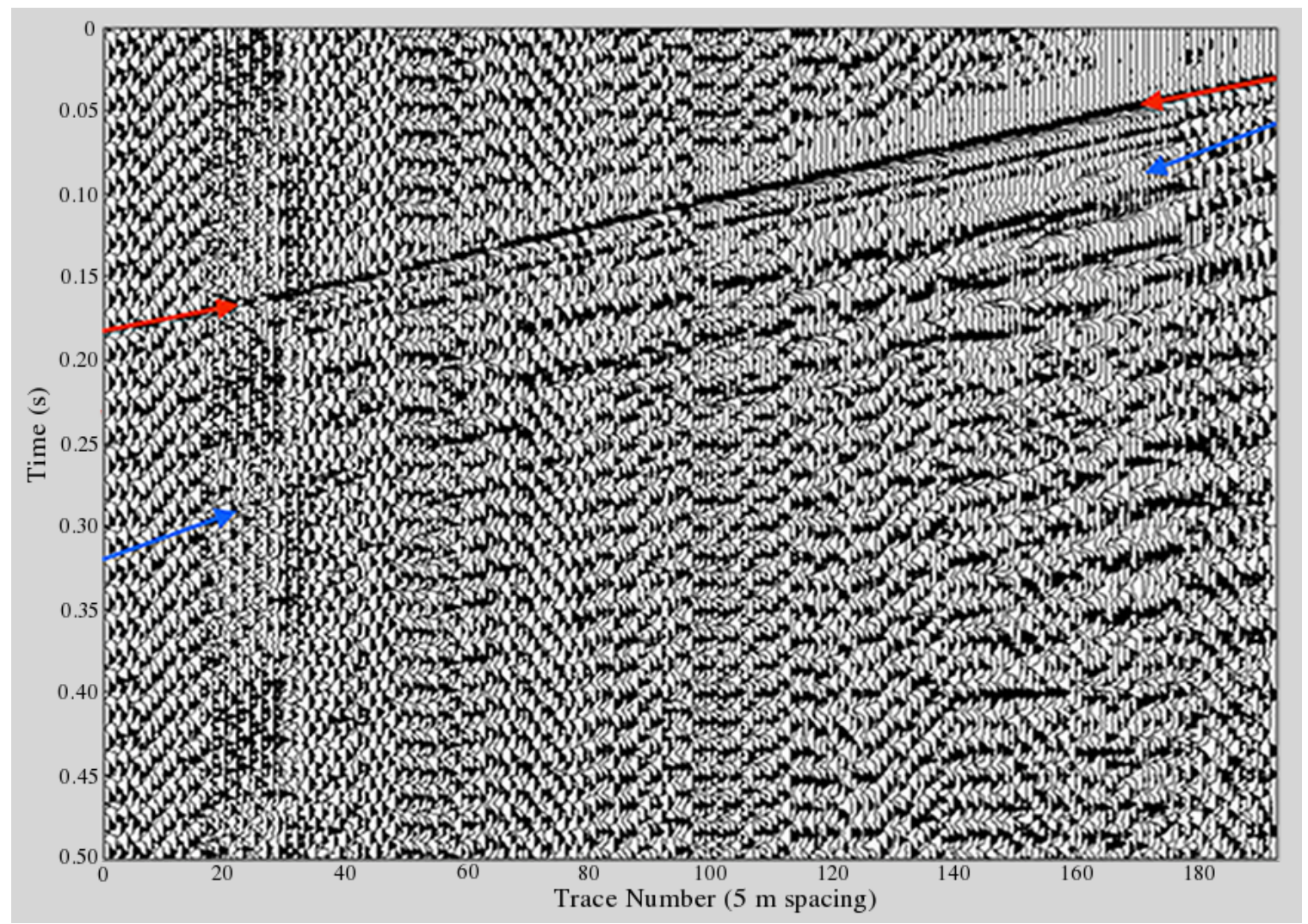

Figure 3.3.2.3 - AGC-scaled vertical component zero-offset P-Vibroseis data for

4Q66W3. The downgoing P-wave is clearly seen and the downgoing S-wave is faint but

identifiable, annotated with red and blue arrows respectively. The signal-to-noise levels are generally lower than for the corresponding dynamite data (cf. Figure 3.3.2.1). 


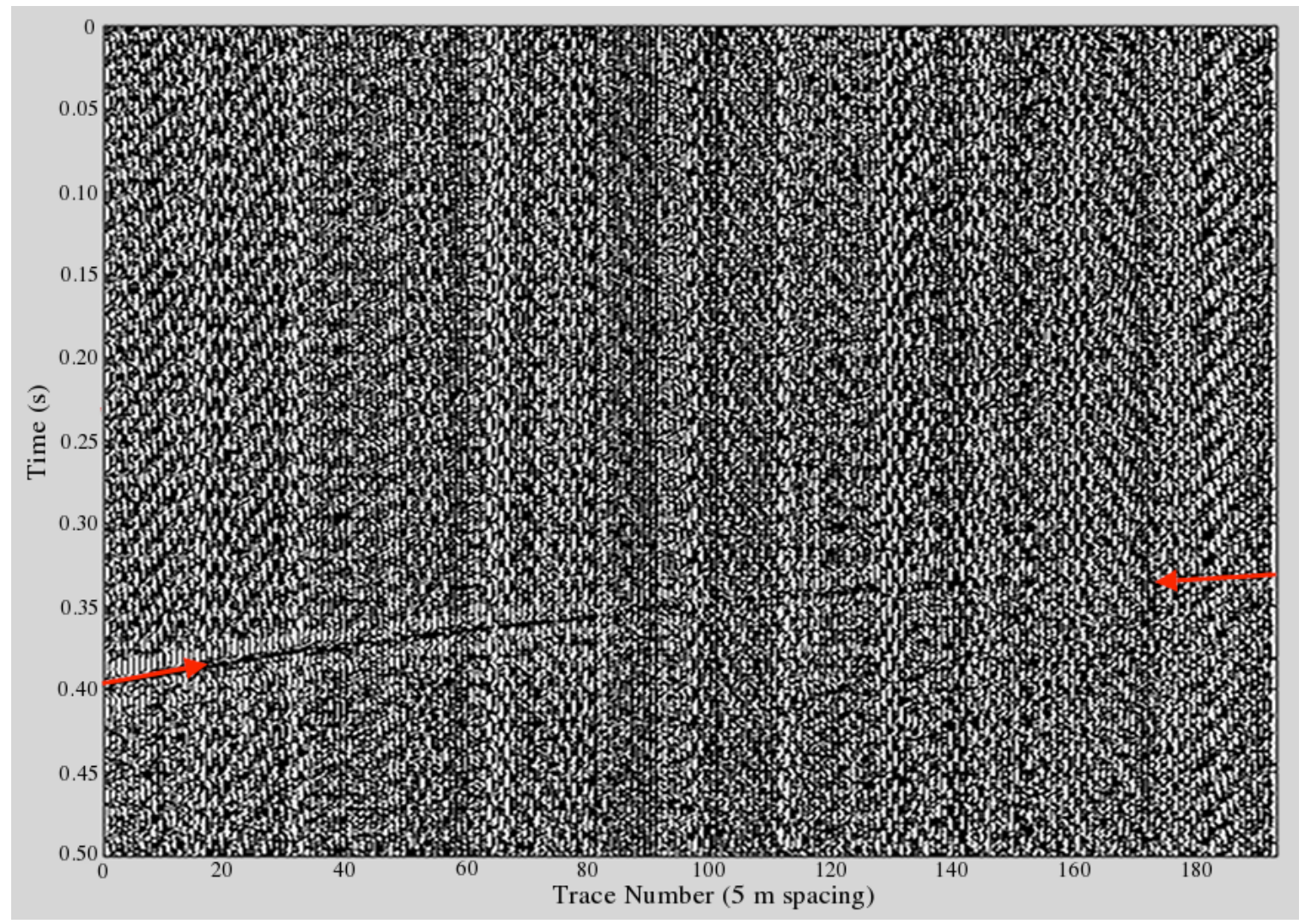

Figure 3.3.2.4 - AGC-scaled vertical component far-offset dynamite data for 4Q66W3.

The downgoing P-wave occurs much later than for the zero-offset data and is clearly seen, annotated with red arrows. 


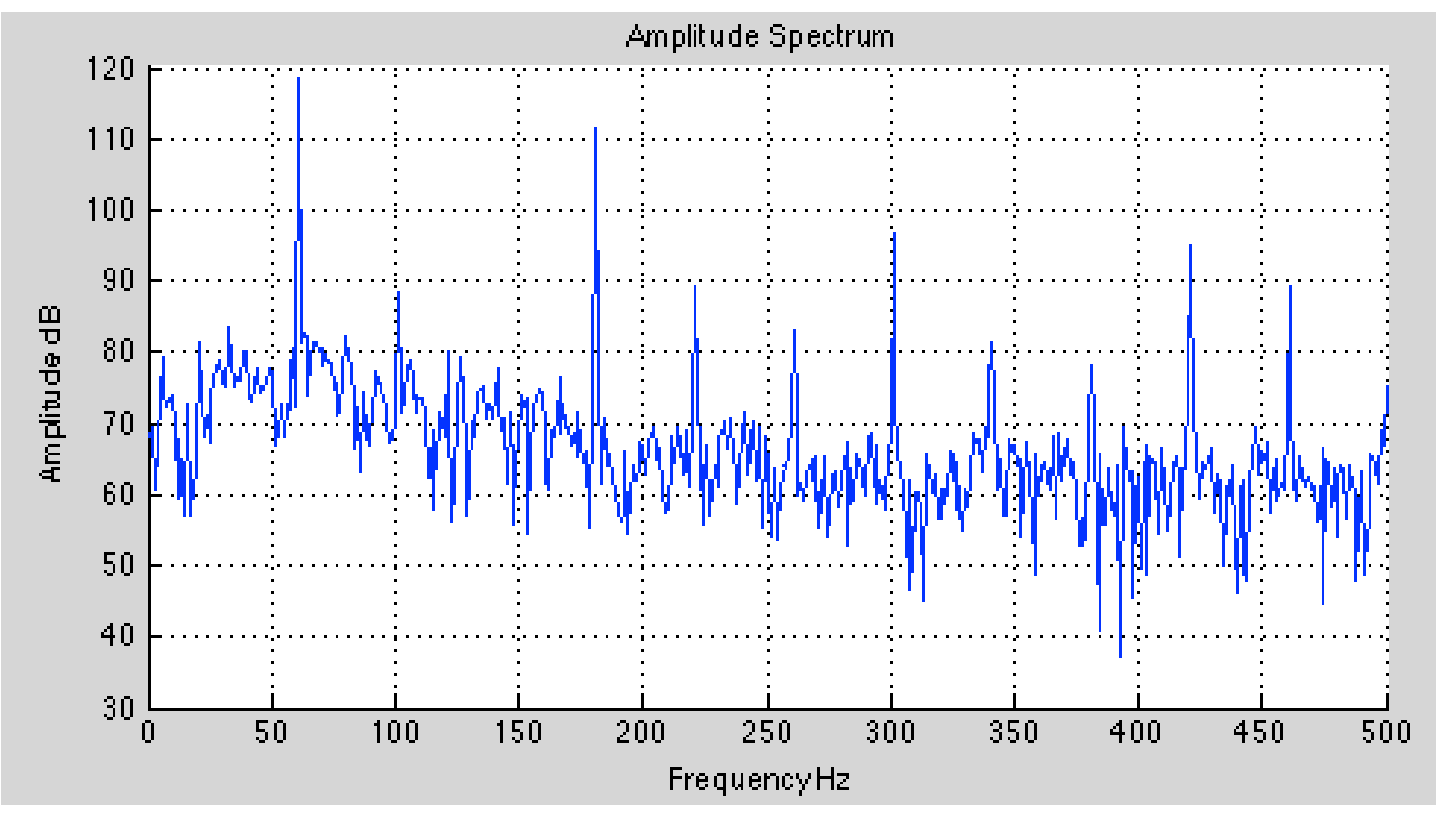

Figure 3.3.2.5 - Frequency vs. Amplitude for all traces of the vertical component P-wave

Vibroseis data shown in Figure 3.3.2.3. Strong peaks exist at $60 \mathrm{~Hz}$ and several associated harmonics representing electrical noise.

As one of the goals of this study is to identify and characterize reflections originating from the known ore lenses, some simple criteria were determined to establish the data sets to prioritize. VSP data from a borehole in close proximity to the known ore lenses would be most likely to contain reflections from those lenses. A higher signal-to-noise ratio in data sets after processing would also contribute to the identification of any ore lens reflections. With those criteria in mind, the 3-component zero-offset dynamite data from 4Q66W3 were chosen as the focus of this study.

The first necessary processing task was to mathematically rotate the horizontal component data into radial and transverse components (Figure 3.3.2.6). This was 
accomplished using DSISoft modules. Initially, due to rotation of the geophone tool within the borehole, unprocessed traces from the horizontal geophones are oriented randomly which isn't practical for interpretation. Also, this geophone tool is not equipped with a compass at each level, which would allow a much simpler operation to align the traces. Using the known azimuth of the far-offset dynamite source from the well collar and both raw horizontal components, the amplitudes of the picked downgoing P-waves for each trace can be mathematically maximized (i.e. into a radial horizontal component oriented toward the source) and minimized (i.e. into a transverse horizontal component oriented perpendicular to the radial) by DSISoft. The azimuths to complete this task are then recorded into respective trace headers and all sets of horizontal component data for that borehole are rotated into similar components. The effectiveness of this process was qualitatively determined to be successful by comparing the strength of the resultant downgoing P-waves for each component in the far-offset dynamite data. This terminology will be used to refer to the rotated horizontal components throughout this study, although the rotations don't correspond to the geometry of other source locations. 


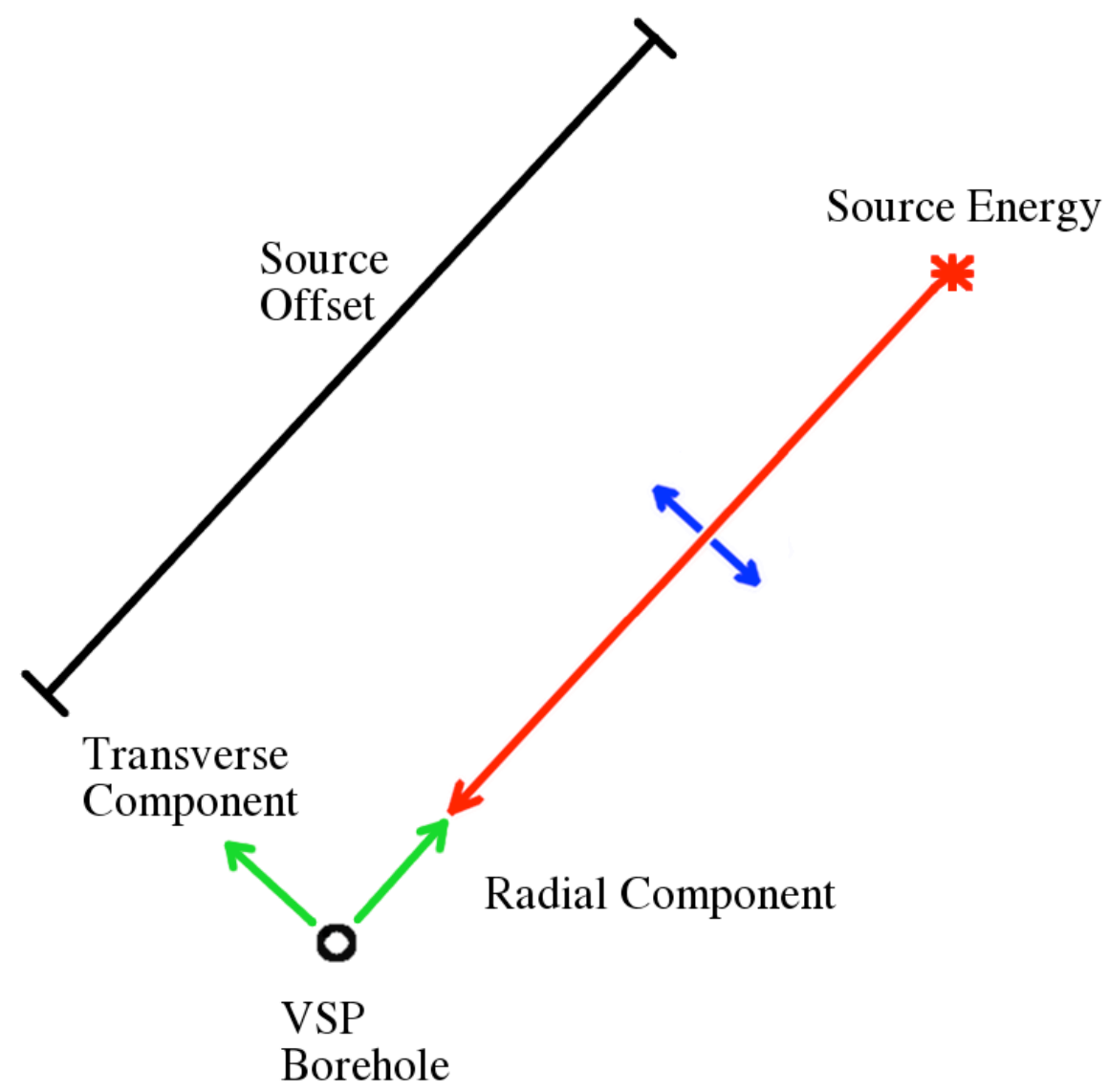

Figure 3.3.2.6 - Plan view demonstration of the far-offset VSP geometry used to mathematically rotate the horizontal geophone components into radial and transverse components. Red arrows illustrate the propagation direction of direct P-waves, blue arrows illustrate the orientations of particle motion for direct S-waves and green arrows illustrate the rotated horizontal components. The maximum amplitude of the direct Pwave would be associated with the radial component.

\subsection{3 - Data processing using Globe Claritas}

Following the rotation process, MATLAB data from DSISoft were exported to SEG-Y format for use with Globe Claritas processing software, which contains a greater number 
of processing tools. Globe Claritas uses multiple graphical user interfaces to import, manipulate in a customizable sequence of processing tools, display and export seismic data files. It runs on a linux cluster operated by the 3D Imaging and Earth Modeling group at the GSC. Each Globe Claritas shot gather was exported and displayed using Seismic Unix as greyscale variable density plots using an AGC with a $250 \mathrm{~ms}$ window. All data sets displayed in this section are the first 0.5 seconds of zero-offset dynamitesource vertical component data from 4Q66W3 unless otherwise noted in the figure captions. Table 3.3.3.1 contains a list of the Globe Claritas processing operations and associated parameters. A brief description of the processing flow with accompanying figures follows.

Table 3.3.3.1 - Globe Claritas processing operations and parameters used to process zerooffset dynamite source VSP data from 4Q66W3. A similar sequence of processing tools was applied to all VSP data from Flin Flon (detailed in Appendix A).

\begin{tabular}{|c|c|c|c|}
\hline $\begin{array}{l}\text { Borehole } \\
\text { Data Set } \\
\text { Component }\end{array}$ & $\begin{array}{l}\text { 4Q66W3 } \\
\text { Zero-offset dynamite } \\
\text { Vertical }\end{array}$ & & \\
\hline PROCESSING STEP & & TOOL & INPUT PARAMETERS \\
\hline \multicolumn{2}{|c|}{ Add first break pick times into trace header } & ADDPICK & Pick file, Trace header \\
\hline \multicolumn{2}{|c|}{ Spiking Wiener deconvolution } & DECONW & Flattened to P-wave picks, $100 \mathrm{~ms}$ operator, $0.1 \%$ pre-whitening \\
\hline \multicolumn{2}{|c|}{ Frequency-domain time and spatially varying filter } & FDFILT & Bandstop: $57-59 \mathrm{~Hz}$ tapered high cut, $61-63 \mathrm{~Hz}$ tapered low cut \\
\hline \multicolumn{2}{|c|}{ Frequency-domain time and spatially varying filter } & FDFILT & Bandstop: $175-179 \mathrm{~Hz}$ tapered high cut, $181-185 \mathrm{~Hz}$ tapered low cut \\
\hline \multicolumn{2}{|c|}{ Frequency-domain time and spatially varying filter } & FDFILT & Bandpass: $10-20 \mathrm{~Hz}$ tapered low cut, $270-300 \mathrm{~Hz}$ tapered high cut \\
\hline \multicolumn{2}{|c|}{ Generalized muting of $f-k$ spectra } & FKMUTE & Flattened to $\mathrm{P}$-wave picks, custom direct $\mathrm{P}$-wave mute file \\
\hline \multicolumn{2}{|c|}{ Generalized muting of $f-k$ spectra } & FKMUTE & Custom direct P-wave mute file \\
\hline \multicolumn{2}{|c|}{ Generalized muting of $f-k$ spectra } & FKMUTE & Custom aliased $\mathrm{P}$-wave mute file \\
\hline \multicolumn{2}{|c|}{ Generalized muting of $f-k$ spectra } & FKMUTE & Custom direct S-wave mute file \\
\hline \multicolumn{2}{|l|}{ Trace balance } & BALANCE & Full trace amplitude balance \\
\hline \multicolumn{2}{|c|}{ FX-domain complex weiner deconvolution } & FX DECON & 12-trace filter applied to 24 traces, $200 \mathrm{~ms}$ window \\
\hline \multicolumn{2}{|c|}{ Mutes to input trace header value } & ZEROMUTE & P-wave picks, $40 \mathrm{~ms}$ cosine taper \\
\hline \multicolumn{2}{|l|}{ Bulk static shift } & BULKSHIFT & $-50 \mathrm{~ms}$ \\
\hline \multicolumn{2}{|l|}{ Automatic gain control } & $\mathrm{AGC}$ & $250 \mathrm{~ms}$ Window \\
\hline
\end{tabular}


As noted before, the raw data is overprinted by strong noise levels, which requires the careful use of processing techniques to attenuate as much background noise as possible while preserving the upgoing wavefield. Careful monitoring of the data before and after each processing operation is essential to determine the effectiveness of each operation and its associated parameters. This is possible using graphical methods to display spectra of amplitude vs. frequency (referred to herein as the amplitude spectrum), frequency vs. wavenumber (referred to herein as the $f-k$ spectrum), or to display the traces as a shot gather. 


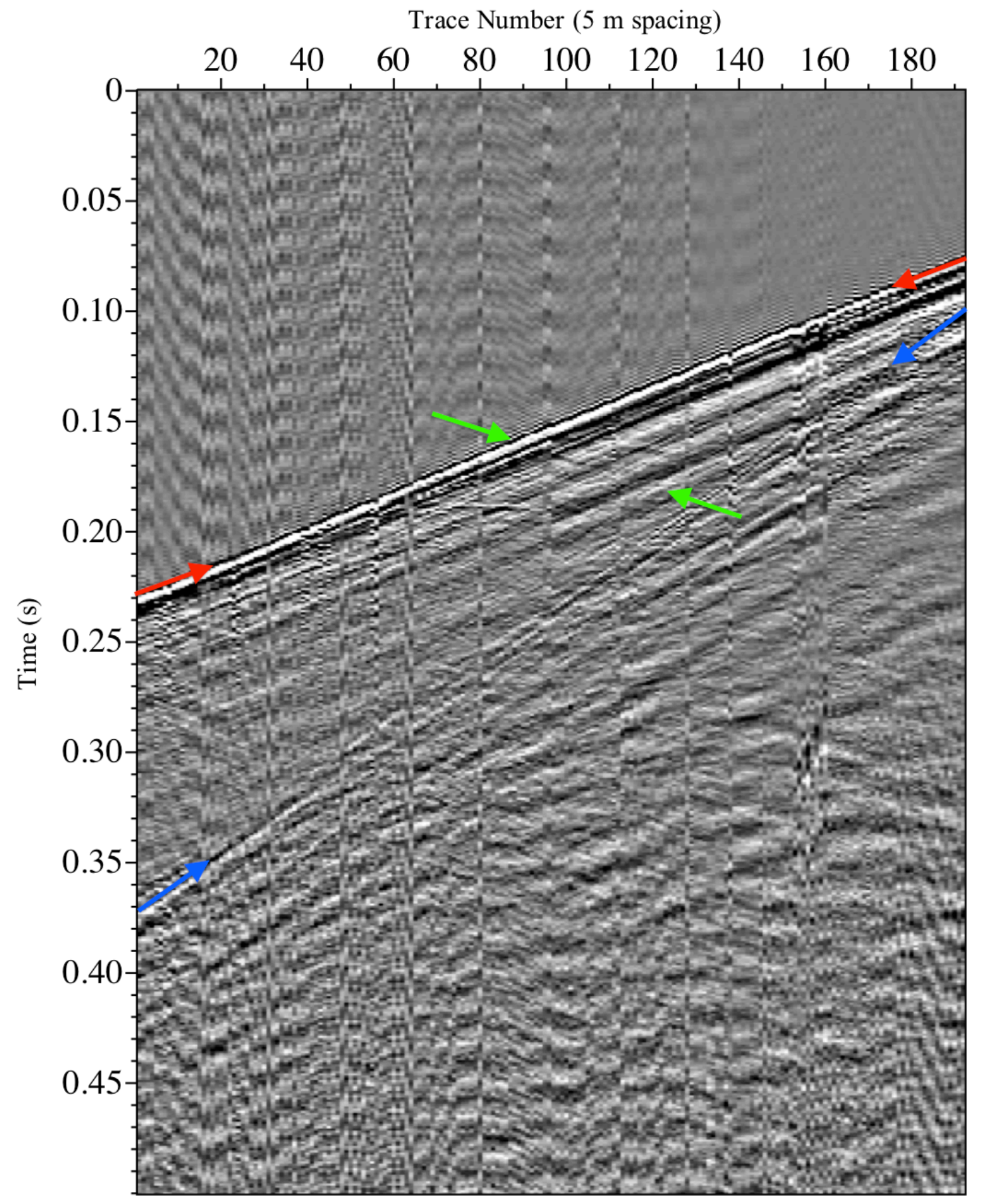

Figure 3.3.3.1 - Raw data as imported into Globe Claritas. The downgoing P- and S-

waves are the most obvious features, annotated with red and blue arrows respectively.

The downgoing P-wave velocity is $\sim 6000 \mathrm{~m} / \mathrm{s}$ and the downgoing S-wave velocity is $\sim$

$3500 \mathrm{~m} / \mathrm{s}$. Some reflections can also be seen and an example with a velocity of $\sim 6400$ 
$\mathrm{m} / \mathrm{s}$ is annotated with green arrows. It should be noted that the colours of arrows representing reflections are not consistent when comparing shot gathers.

Before processing, it is necessary to visually pick the first break of the downgoing Pwave for each trace and save them in trace headers to be used later in several of the processing steps. The first operation in the sequence is a spiking Wiener deconvolution. Each trace is shifted such that the first breaks appear to be traveling infinitely fast (i.e. they are flat horizontally). Spiking deconvolution autocorrelates each trace using an input lag of one sample to form a predicted output at that lag. The predicted output is then cross-correlated with the input trace in attempt to compress complex reverberatory waveforms into sharp spikes. The data is then shifted back to the original time (Figure 3.3.3.2). 


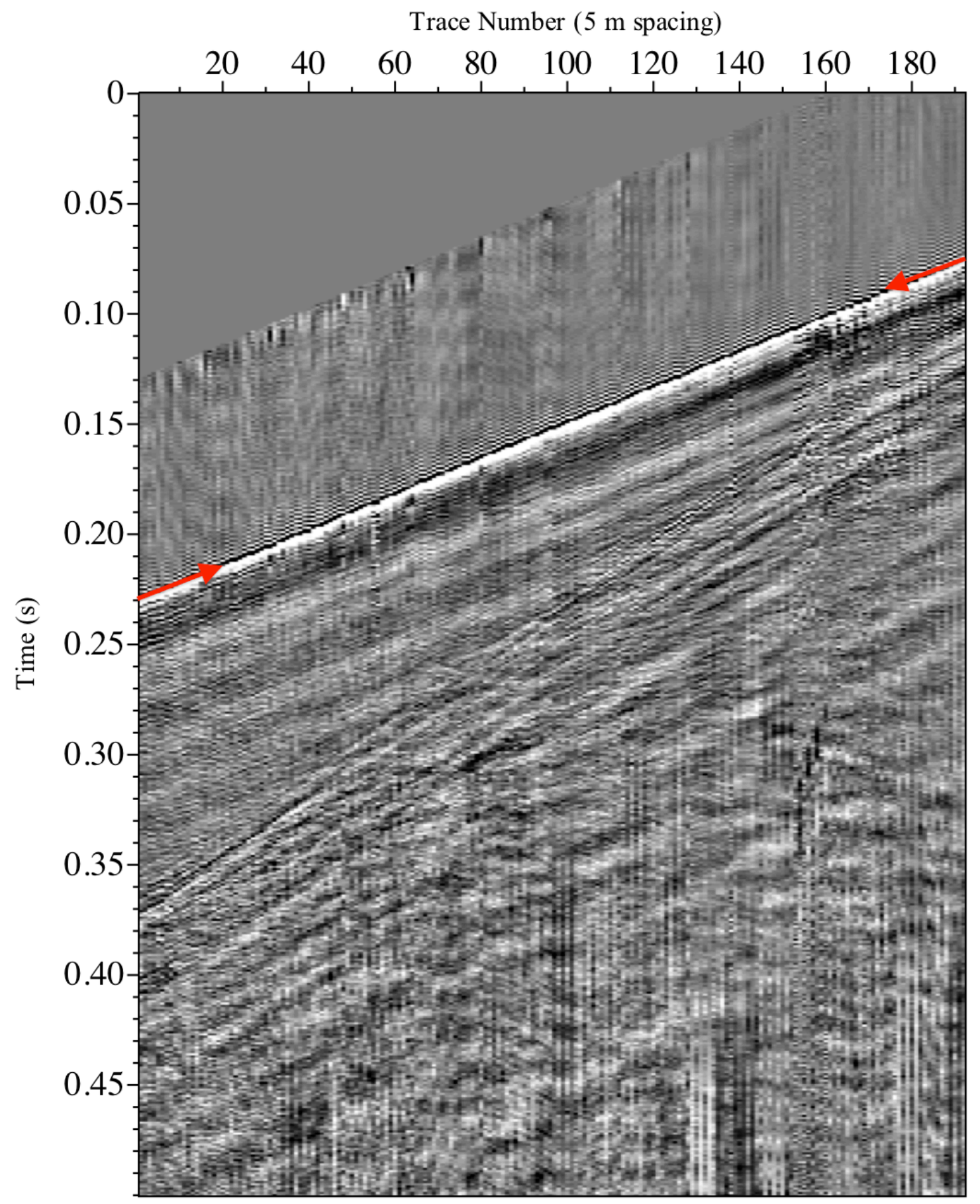

Figure 3.3.3.2 - Data after spiking deconvolution. The downgoing P-wave (red arrow)

has been compressed compared to the complex waveform seen in the raw data (cf. Figure $3.3 .3 .1)$ 


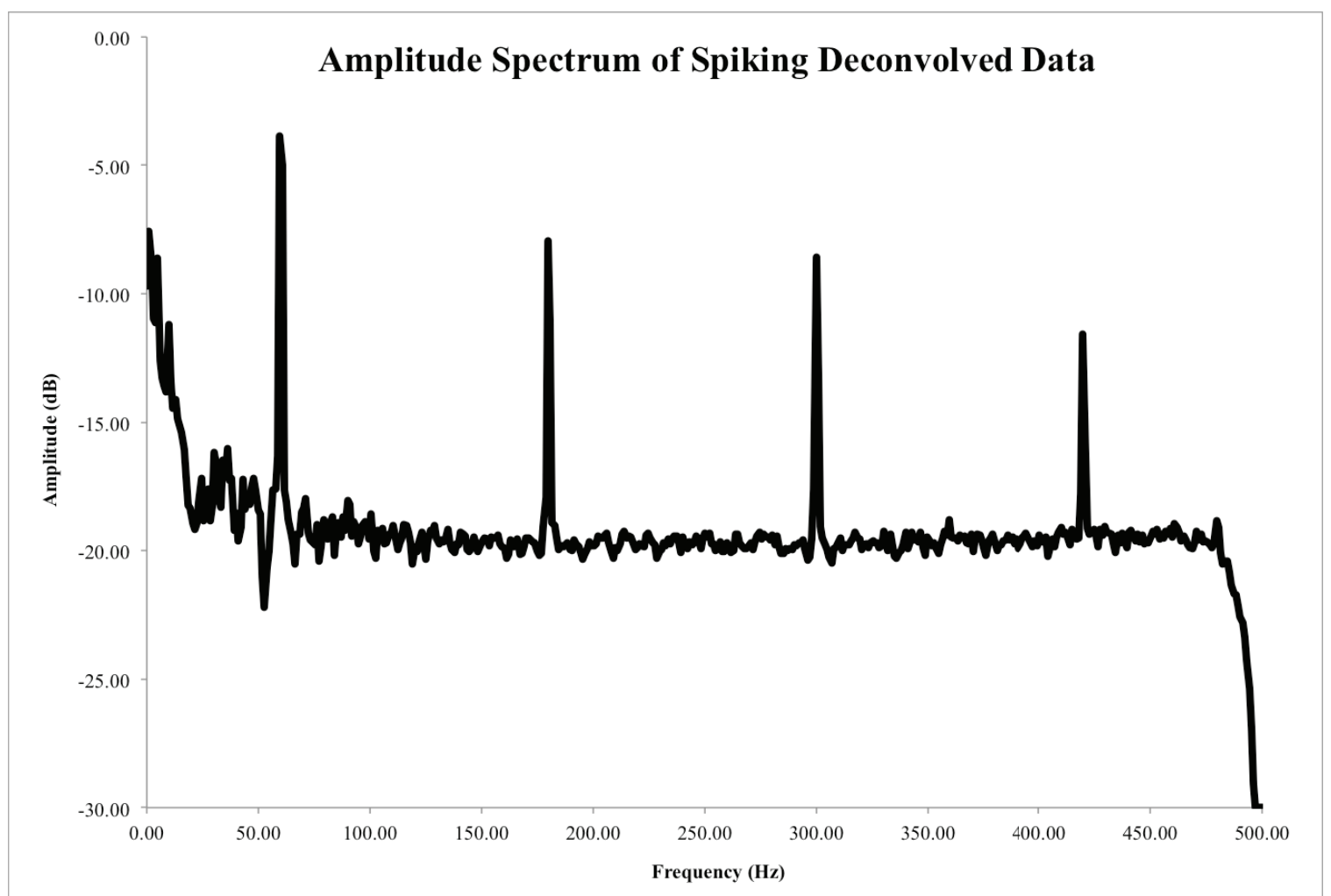

Figure 3.3.3.3 - Amplitude spectrum of data after spiking deconvolution. Peaks at 60, 180, 300 and $440 \mathrm{~Hz}$ representing electrical noise are seen.

The most obvious characteristics of the amplitude spectrum of the data after spiking deconvolution shown in Figure 3.3.3.3 are the strong narrow peaks centered around 60, 180,300 and $440 \mathrm{~Hz}$, representing strong electrical noise throughout the data.

Customized tapered notch filters were then applied to the 60 and $180 \mathrm{~Hz}$ peaks to reduce the amplitude of the electrical noise (Figure 3.3.3.4 and 3.3.3.5). The 300 and $440 \mathrm{~Hz}$ peaks were left to be removed by a bandpass filter, which attenuates frequencies outside a specified and tapered set of frequencies. The downgoing signal was found to be between 20 and $270 \mathrm{~Hz}$ so these values were chosen as the corner frequencies of the pass band (Figure 3.3.3.6). 


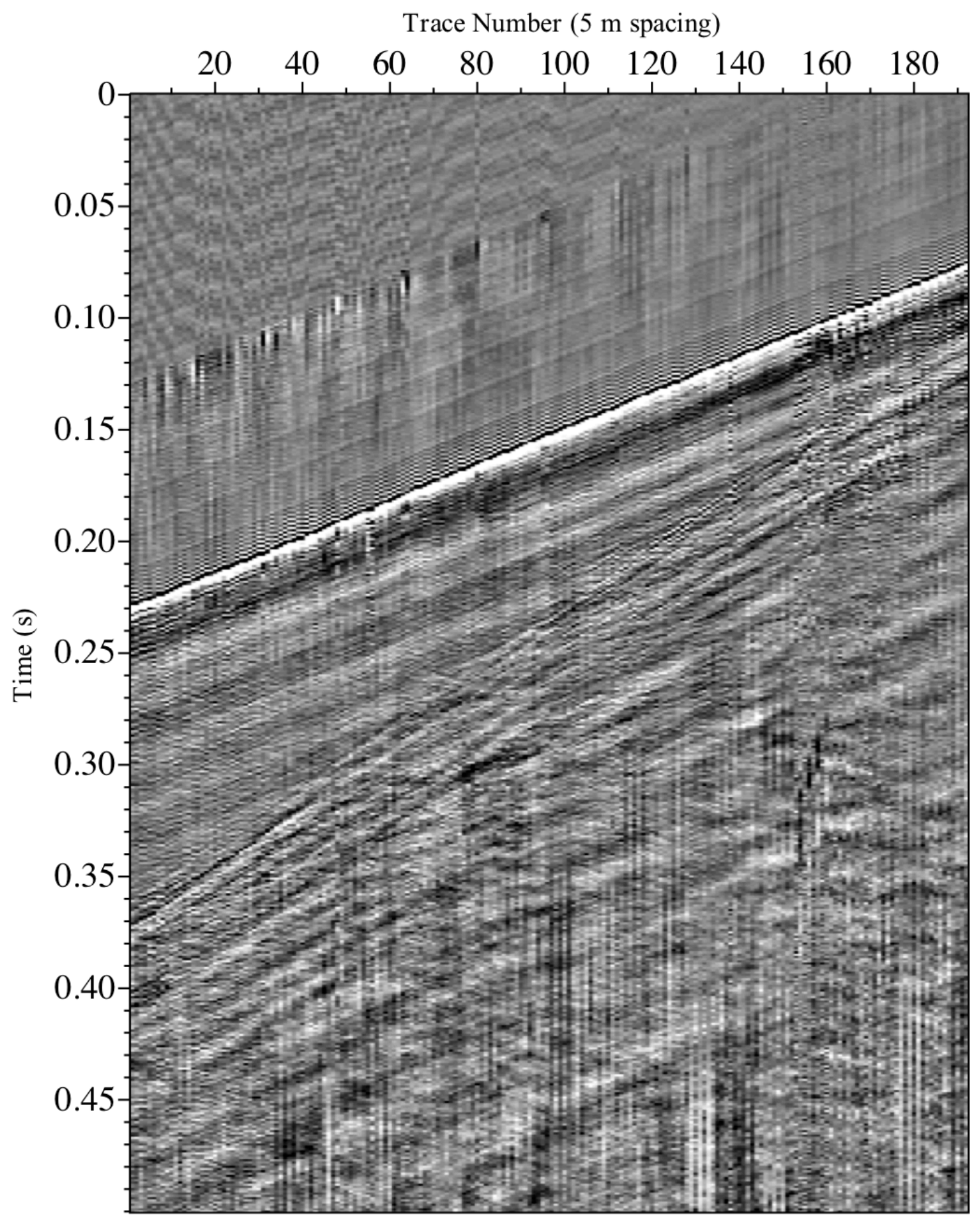

Figure 3.3.3.4 - Notch filtered data. 


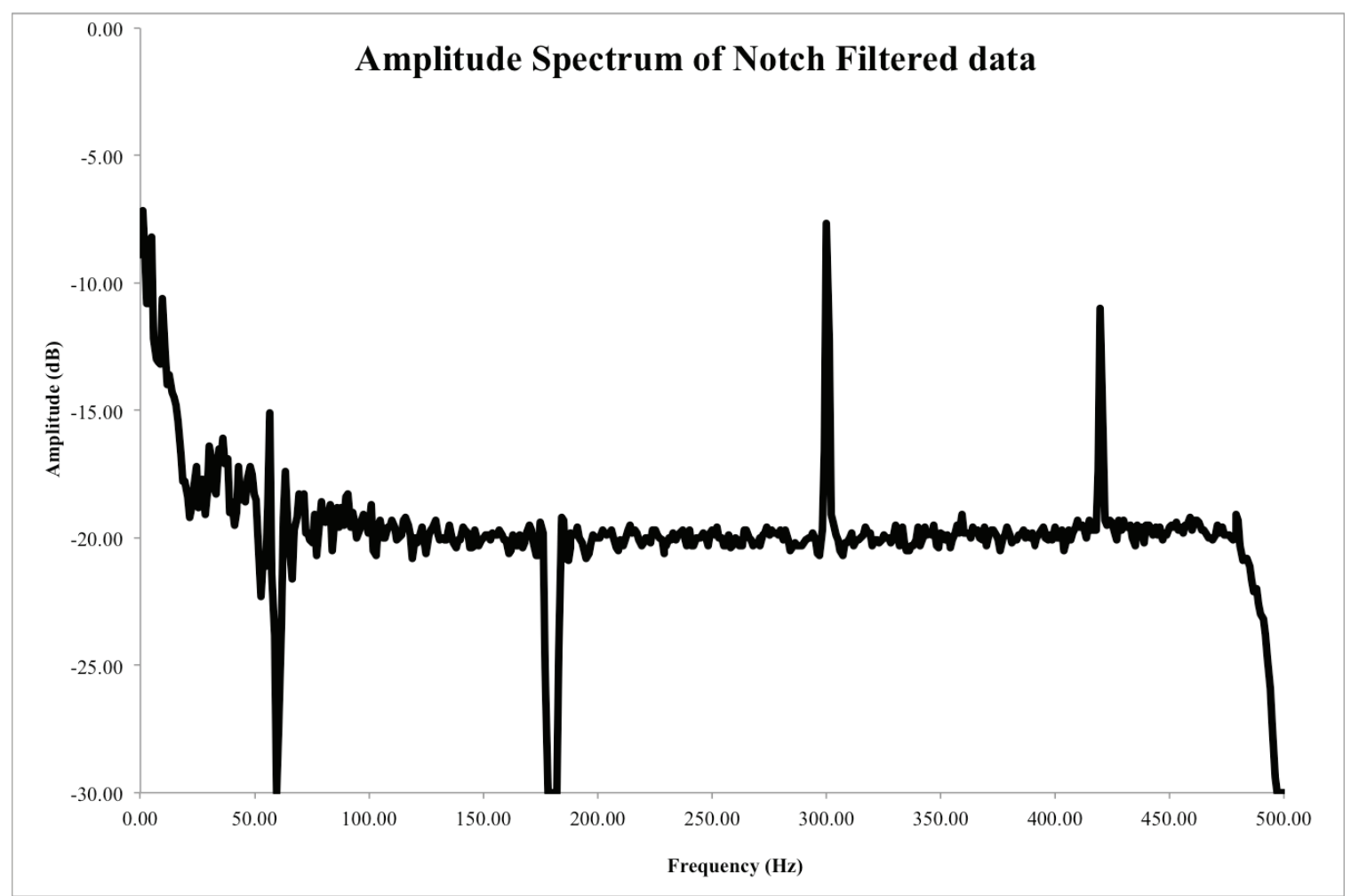

Figure 3.3.3.5 - Amplitude spectrum of notch filtered data. Previously-mentioned peaks

at 60 and $180 \mathrm{~Hz}$ have been reduced. Peaks at 300 and $440 \mathrm{~Hz}$ will be removed by the bandpass filter. 


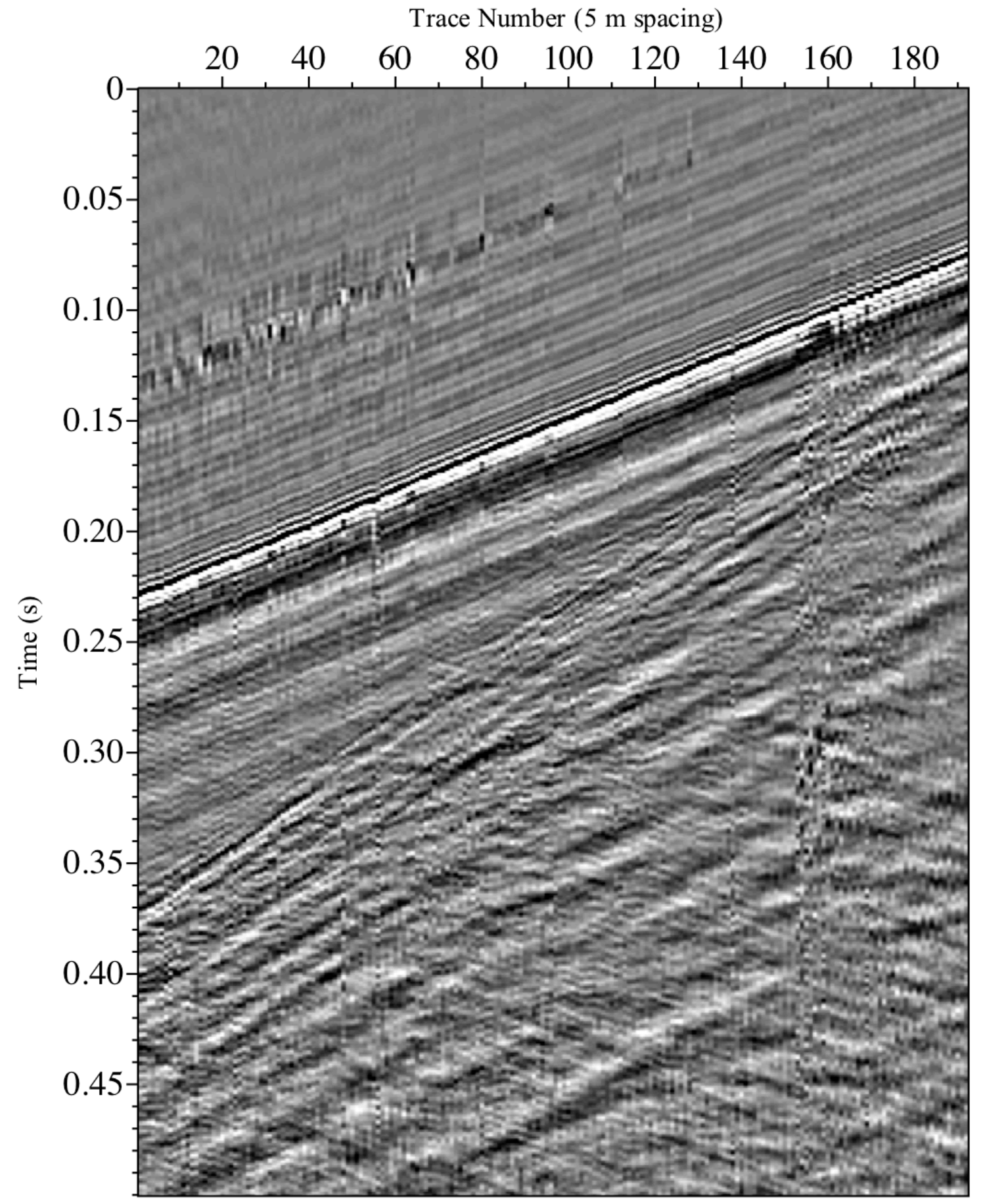

Figure 3.3.3.6 - Bandpass filtered data. The tapered low cut is between 10 and $20 \mathrm{~Hz}$ and the tapered high cut is between 270 and $300 \mathrm{~Hz}$. Considerably more detail after the downgoing waves can now be seen when compared to the previous figures. 


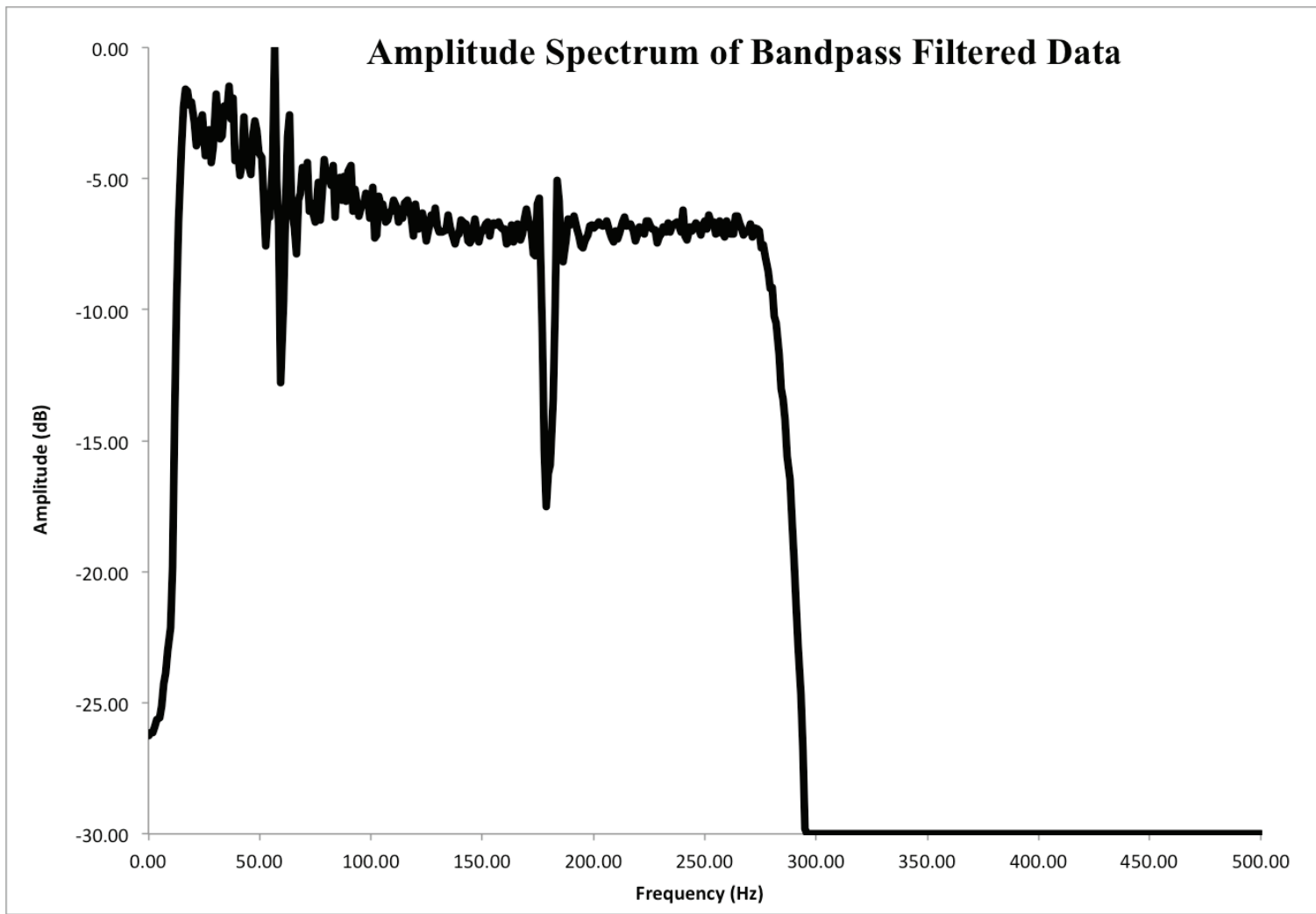

Figure 3.3.3.7 - Amplitude spectrum of bandpass filtered data. The effect of the bandpass filter is shown, with frequencies below $20 \mathrm{~Hz}$ and above $270 \mathrm{~Hz}$ drastically attenuated. 


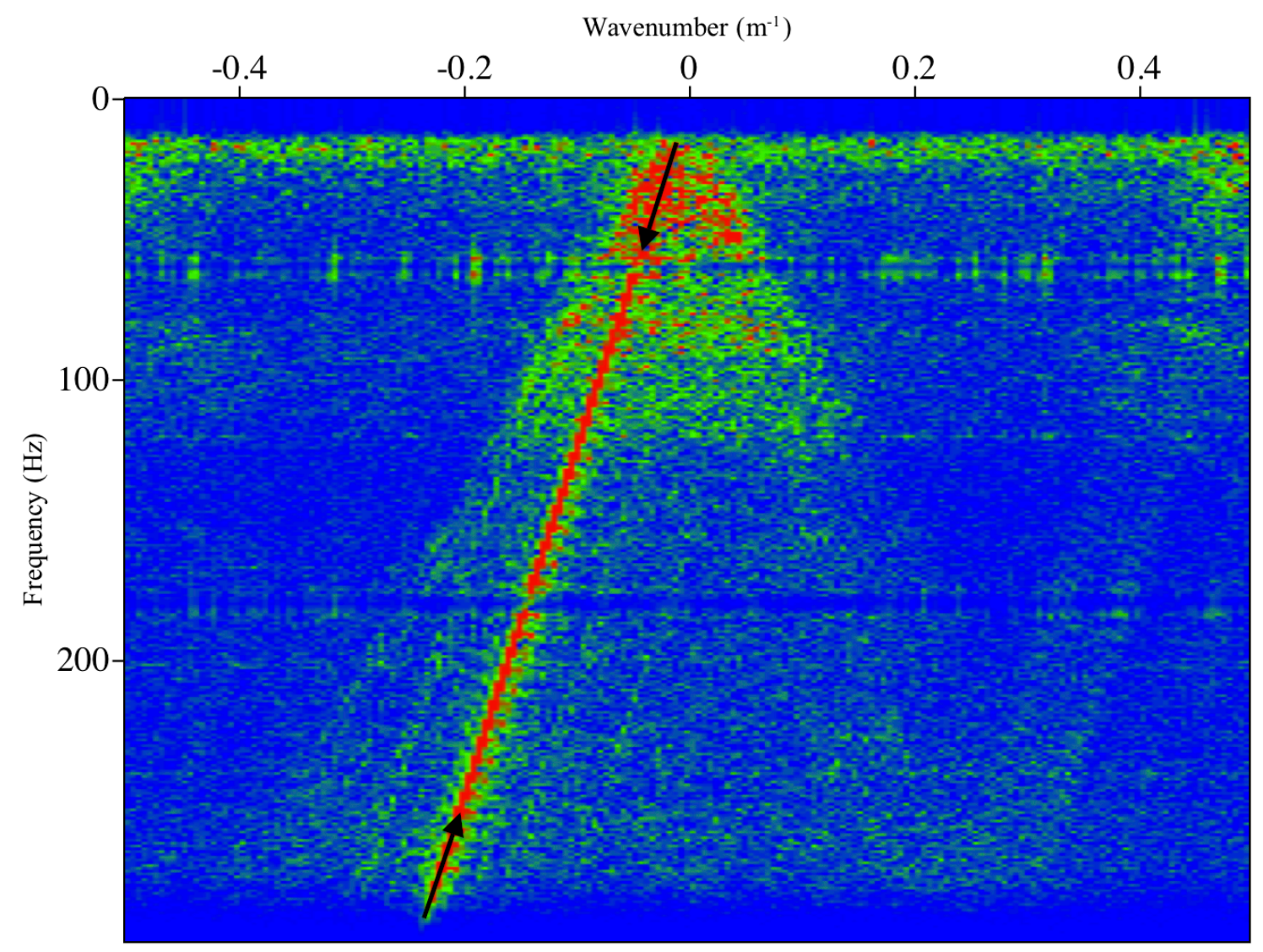

Figure 3.3.3.8 - $f-k$ spectrum of bandpass filtered data. Amplitude strength increases from blue-to-green-to-red. A very strong band of energy with negative slope can be seen which represents the downgoing P-wave (black arrows). Faint horizontal bands of leftover 60 and $180 \mathrm{~Hz}$ electrical noise can also be seen.

The next operation to be applied was $f-k$ filtering. First, a Fourier transform was used to convert data to the frequency vs. wavenumber domain and the spectra displayed. This spectrum is very helpful in separating downgoing and upgoing energy by displaying bands of energy with negative and positive slopes, respectively. A custom window was then selected around bands to be removed. In this case, three separate bands were identified to be removed, based on their apparent velocity. A very strong, negatively- 
sloping zone represents the downgoing P-wave and was the first to be removed (Figure 3.3.3.8). Then, a region with the same slope but shifted to a higher wavenumber was removed, corresponding to aliased downgoing P-wave energy. Lastly the downgoing shear waves were removed which are also negatively-sloping but not as steep in the $f-k$ domain because their apparent velocity is lower in the time domain. These steps were applied sequentially to visually monitor the efficiency of each filter and to not attenuate any of the upgoing wavefield (Figure 3.3.3.9 and 3.3.3.10). Following this, the traces were balanced horizontally by scaling each trace by a slowly-varying or constant value over time, effectively balancing the average amplitude of all traces (Figure 3.3.3.11). 


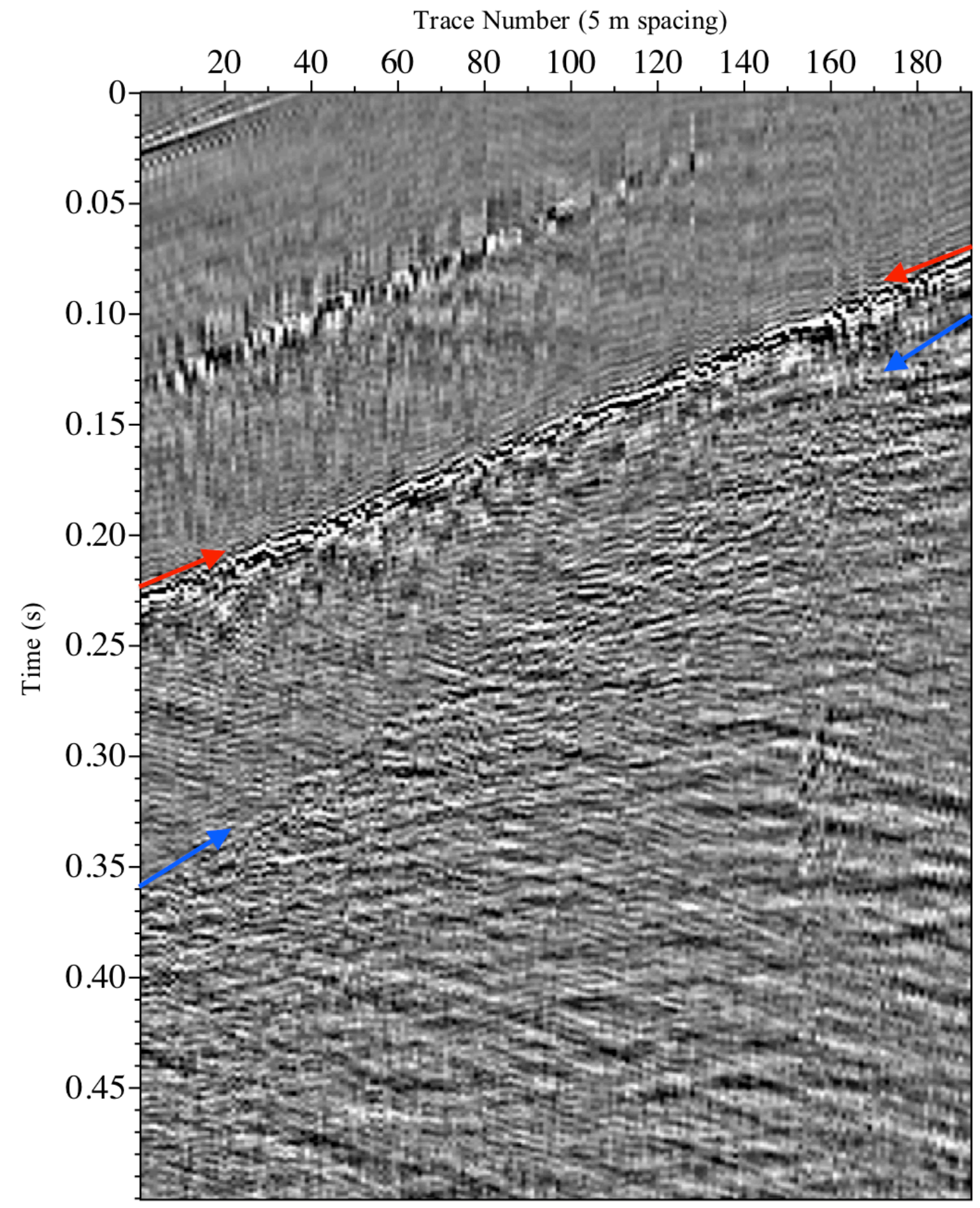

Figure 3.3.3.9 - $f$ - $k$ filtered data. The red and blue annotations for P- and S-waves are

again shown and a significant reduction in their strengths relative to other events can be seen. 


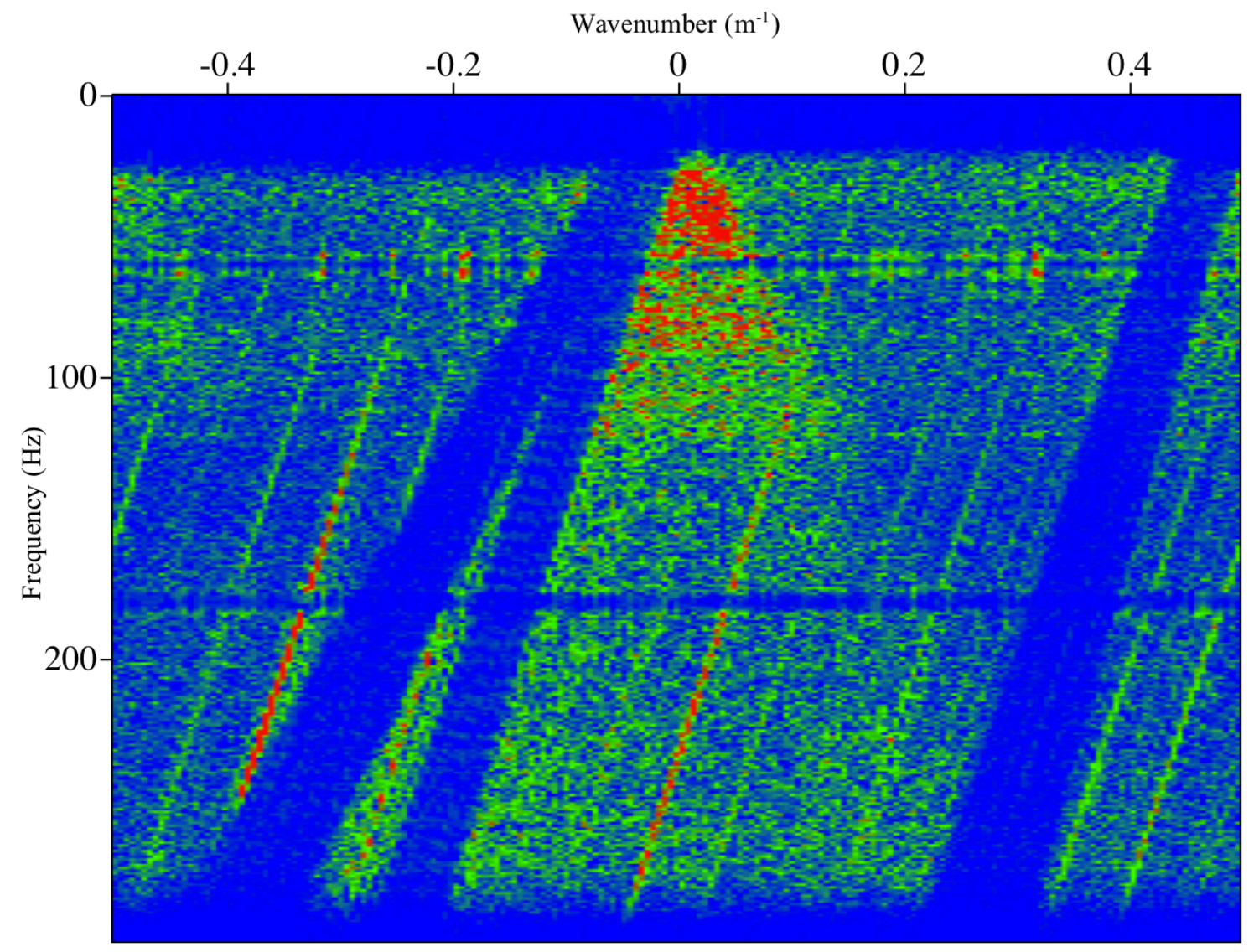

Figure 3.3.3.10 - $f-k$ spectrum of $f-k$ filtered data. 3 mute windows for the downgoing waves can be seen. Several more red-coloured bands of aliased P-wave energy can now be seen as well, which were ignored at this stage because their removal could unintentionally attenuate the upgoing wavefield. 


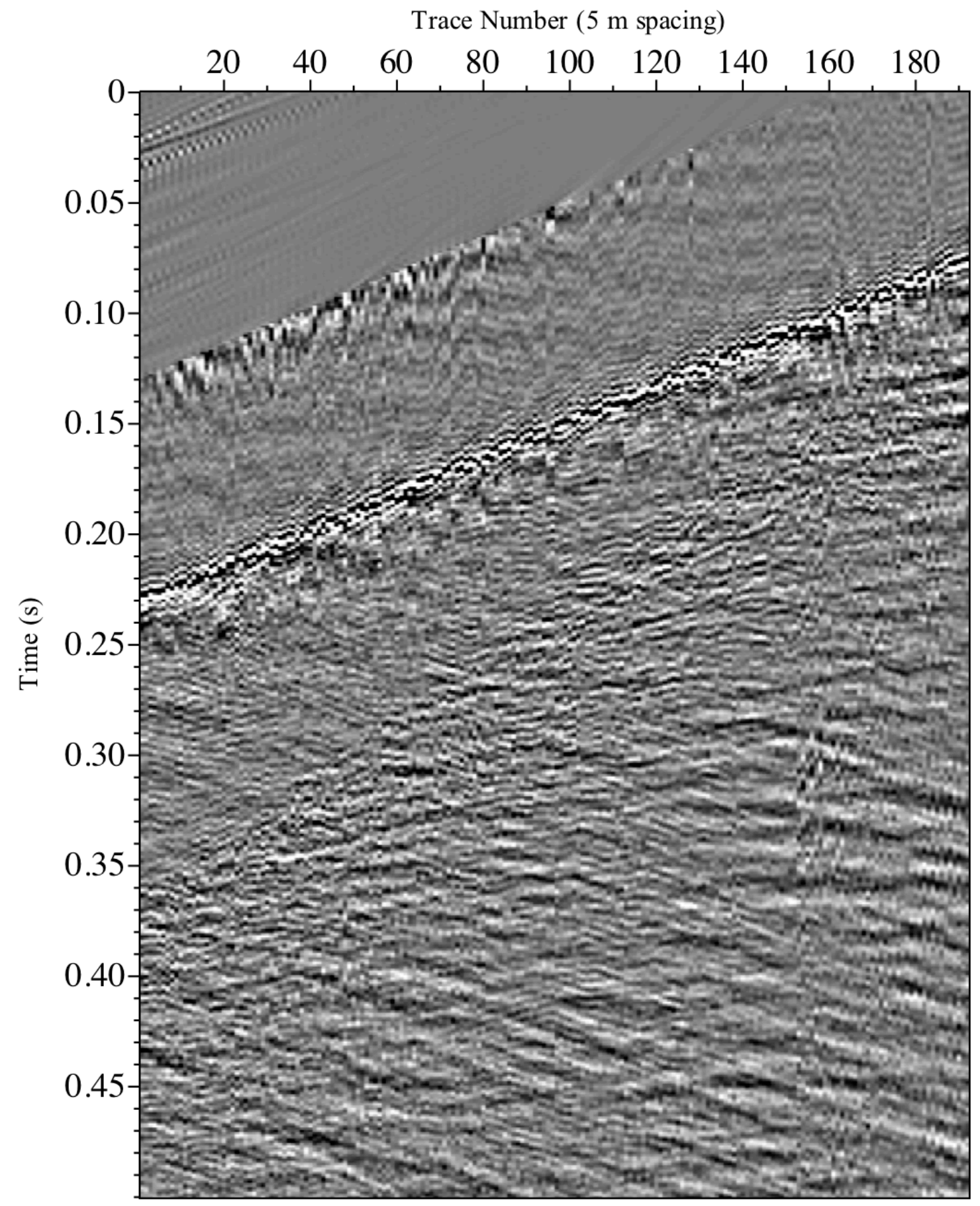

Figure 3.3.3.11 - Trace balanced data.

The final processing operation applied was FX deconvolution (Figure 3.3.3.12). Each trace is transformed into the frequency vs. geophone spacing $(f-x)$ domain and undergoes 
complex Weiner deconvolution within frequency windows. The filtered trace is then transformed back into time vs. geophone spacing $(t-x)$ space for display. FX deconvolution was used to attenuate random noise and leaves a shot gather which looks less synthetic than other methods. Next, a tapered mute was applied to all traces up to 40 ms after the first breaks to completely remove the downgoing P-waves. A final $50 \mathrm{~ms}$ static shift was applied to correct a known trigger delay affecting all of the dynamite data in this study.

Processing of the zero-offset dynamite-source vertical component data from 4Q66W3 has shown a noticeable enhancement of the upgoing wavefield when compared to the raw data. Several continuous reflections with varying apparent velocities can be seen throughout the data, which may originate from nearby contacts with known ore lenses (Figure 3.3.3.13). The amplitude spectrum (Figure 3.3.3.14) shows considerable reduction in the $60 \mathrm{~Hz}$ electrical noise and associated harmonics. The $f-k$ spectrum (Figure 3.3.3.15) shows two faint horizontal bands corresponding to leftover electrical noise but most of the bright colours representing the highest amplitudes have a positive wavenumber and represent the upgoing wavefield. The identified reflections are still quite complex with fluctuating amplitudes and highly variable apparent velocities. 


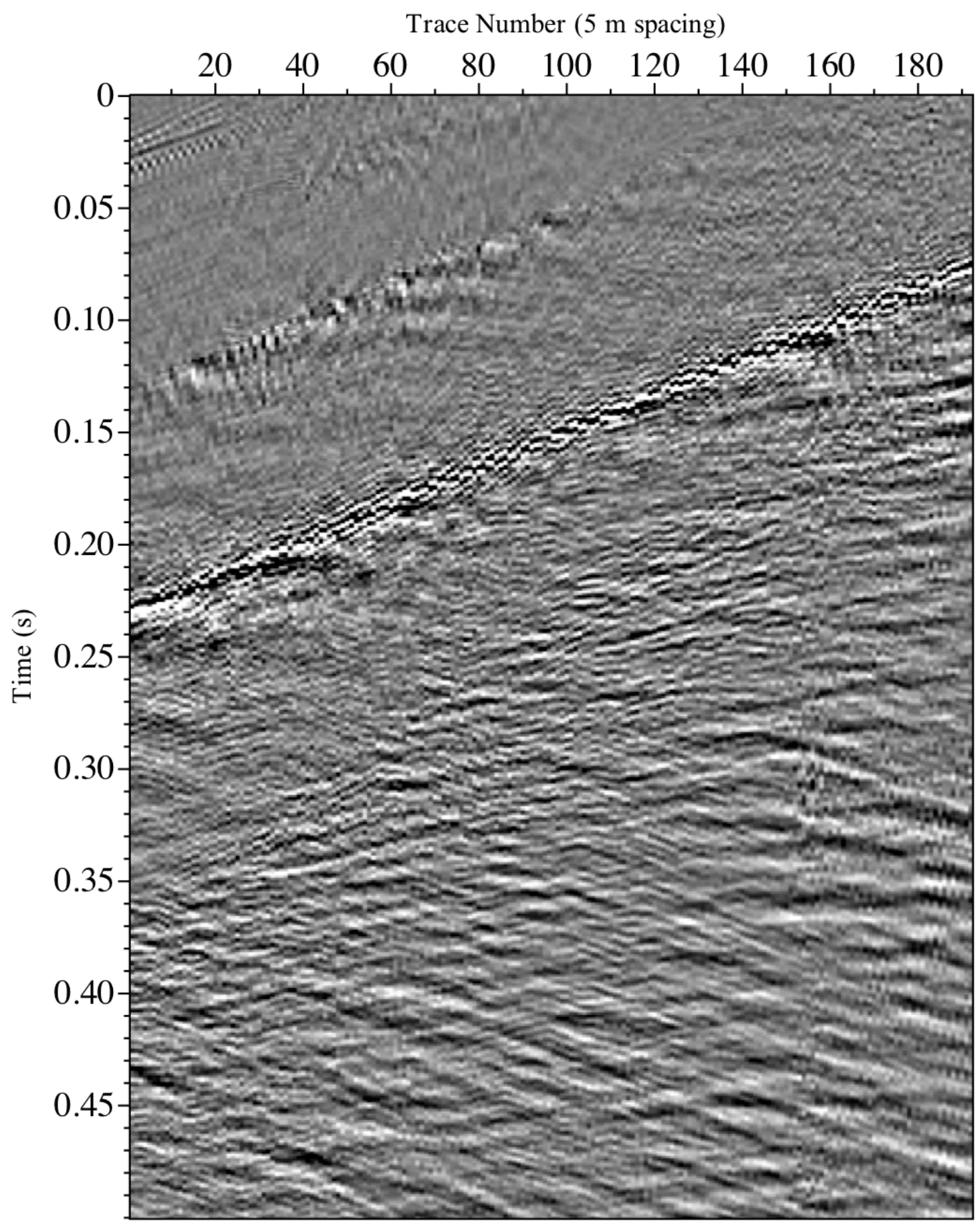

Figure 3.3.3.12 - FX deconvolution filtered data. 


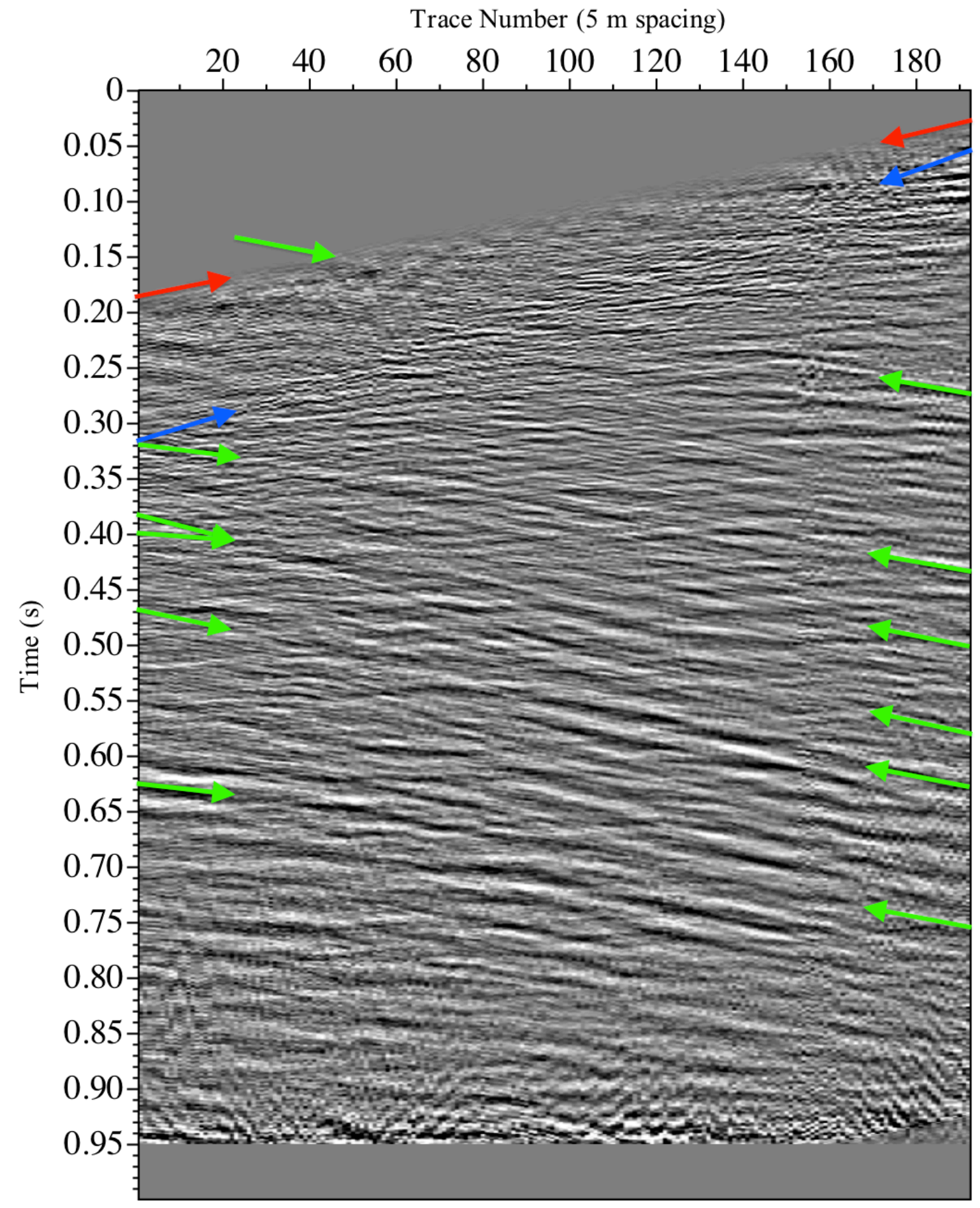

Figure 3.3.3.13 - Final processed data down to $1.0 \mathrm{~s}$. The downgoing P- and S-waves

have been almost completely removed and are annotated with red and blue arrows

respectively. Several continuous upgoing reflections can be seen down to $\sim 0.75 \mathrm{~ms}$

which are annotated with green arrows. 


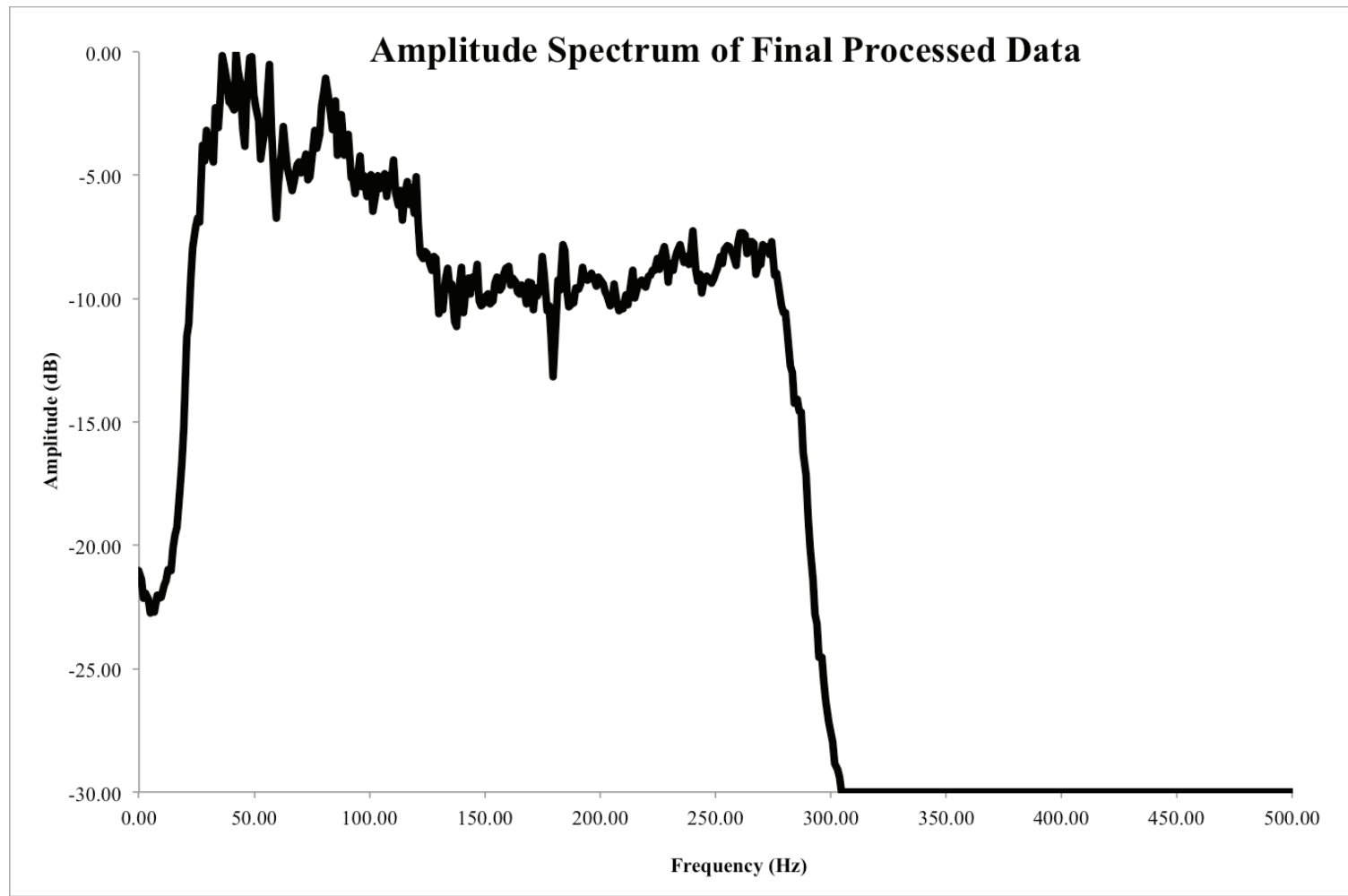

Figure 3.3.3.14 - Amplitude vs. frequency spectrum of the final processed data. 


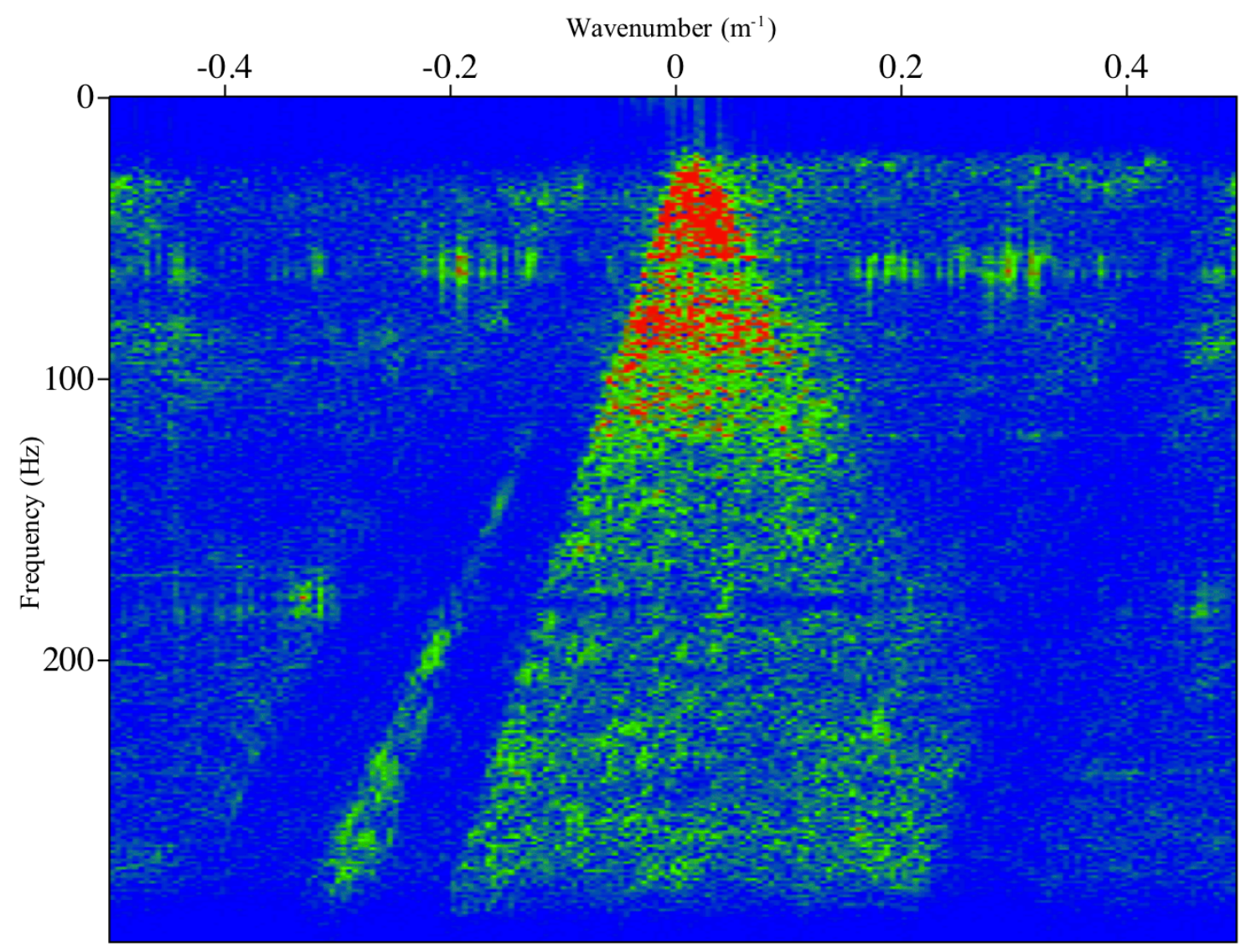

Figure 3.3.3.15 $-f-k$ spectrum of final processed data. Most of the red-coloured area now represents the upgoing wavefield. 


\section{4 - VSP Observations}

Of all the VSP data collected from 4Q66W3, the zero-offset dynamite source data was the highest in initial quality and showed the most improvement through processing. Images of all other data sets can be found in Appendix B. The 3-components of processed zerooffset dynamite data from 4Q66W3 are shown in Figure 3.4.1 (vertical component), Figure 3.4.2 (transverse component) and 3.4.3 (radial component). For each component, the shape, timing and apparent velocity of annotated reflections is documented (Tables 3.4.1, 3.4.2 and 3.4.3), allowing quick comparison with modeled results.

Since 4Q66W3 does not intersect any of the ore lenses, corresponding reflections will display an apparent velocity in the shot gathers that is controlled by the $3 \mathrm{D}$ geometry of the reflectors and the receiver array. This, along with the observed difference in velocity between downgoing P- and S-waves $(6150 \mathrm{~m} / \mathrm{s}$ and $3860 \mathrm{~m} / \mathrm{s}$, respectively) contributes to potential P-P, P-S, S-S or S-P reflections having several different slopes in the $t-x$ (timeoffset) domain. In general, reflections in the field VSP data are discontinuous and can be difficult to trace across the image. Some reflections in each image have been annotated with coloured arrows to aid the viewer, but these annotations are certainly only qualitative. Apparent velocities of all annotated direct and reflected waves have been measured in Globe Claritas using a linear ruler tool, offsets and times. Reflections originating near the borehole can be identified due to their intersection with the downgoing P-wave. The trace at which this intersection occurs identifies the depth of the 
reflector along the borehole. Reflections occurring after the downgoing P-wave reaches the end of the receiver array must originate either from underneath or some distance away from the borehole. 


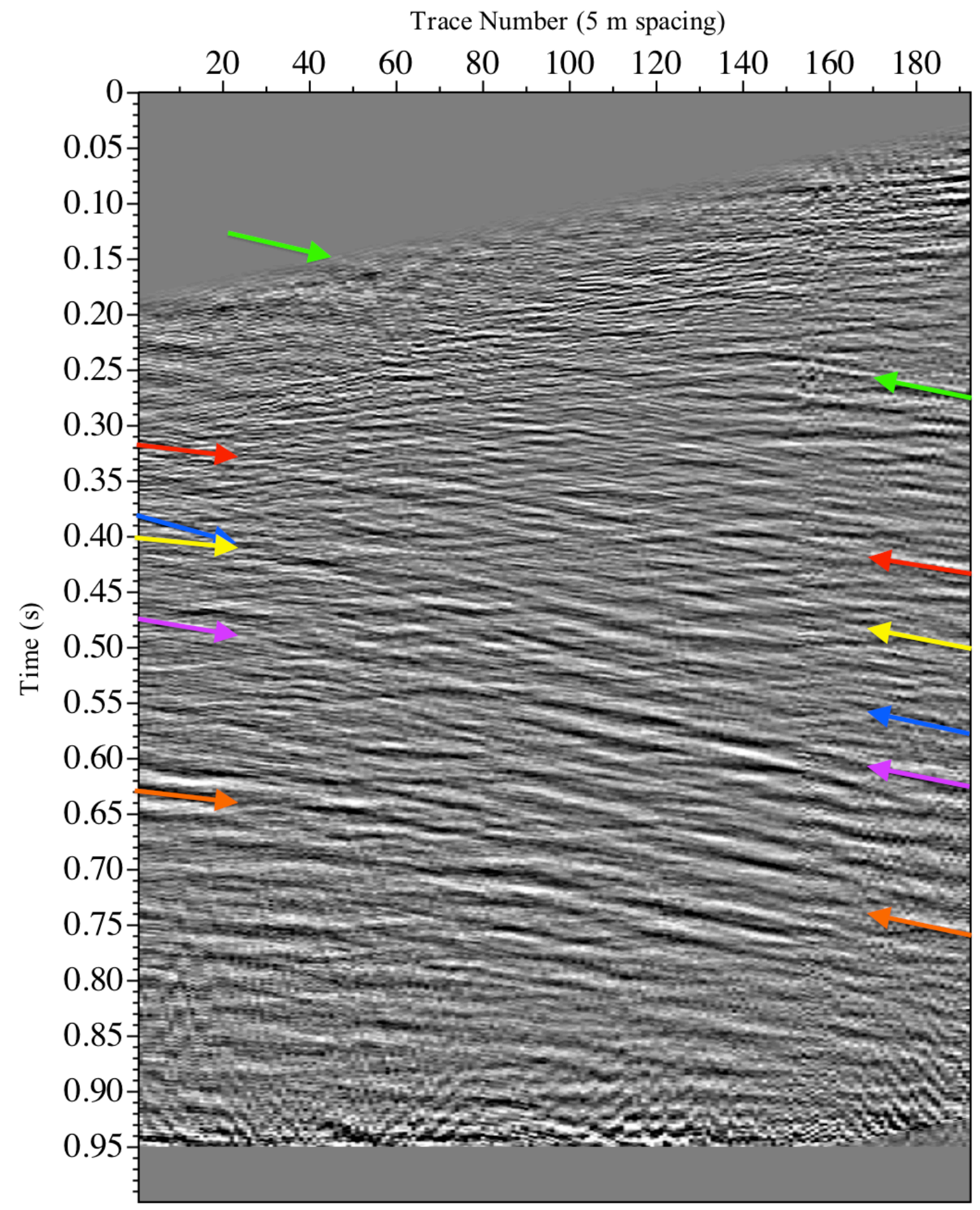

Figure 3.4.1 - Processed zero-offset dynamite source vertical component VSP data from 4Q66W3 with prominent reflections identified by coloured arrows. 
Table 3.4.1 - Summary of annotated events in the zero-offset dynamite source vertical component VSP shot gather shown in Figure 3.4.1.

\begin{tabular}{|c|c|c|c|c|c|c|c|}
\hline \begin{tabular}{|l|} 
Borehole \\
Data set \\
Component \\
Figure \\
\end{tabular} & \begin{tabular}{|l} 
4Q66W3 \\
Zero-offset dynami \\
Vertical \\
3.4 .1 \\
\end{tabular} & & & & & & \\
\hline Arrow Colour & Direct / Reflected & Alignment & Continuity & $\begin{array}{c}\text { Trace } 1 \\
\text { (s) }\end{array}$ & \begin{tabular}{|c|} 
Trace 192 \\
(s)
\end{tabular} & \begin{tabular}{|c|}
$\begin{array}{c}\text { Apparent velocity } \\
(\mathrm{m} / \mathrm{s})\end{array}$ \\
\end{tabular} & Notes \\
\hline Green & Reflection & linear & discontinuous & 0.108 & 0.268 & 6450 & intersects $886 \mathrm{~m}$ \\
\hline Red & Reflection & linear & semi-continuous & 0.325 & 0.430 & 8990 & \\
\hline Blue & Reflection & linear & discontinuous & 0.382 & 0.573 & 4930 & \\
\hline Yellow & Reflection & curved & discontinuous & 0.402 & 0.501 & 9560 & \\
\hline Purple & Reflection & linear & continuous & 0.473 & 0.625 & 6190 & \\
\hline Orange & Reflection & curved & continuous & 0.629 & 0.760 & 7230 & \\
\hline
\end{tabular}

The processed vertical component data set shows no direct P-wave energy, very little direct S-wave energy remaining and several semi-continuous upgoing reflections. A linear reflection can be seen intersecting the borehole (green arrows in Figure 3.4.1) with an apparent velocity of $6450 \mathrm{~m} / \mathrm{s}$ which intersects trace 45 at a depth of $886 \mathrm{~m}$ and reaches the uppermost geophone level at $0.268 \mathrm{~ms}$. This observation is consistent with a previously-identified event in the same data (White et al., in press). The strongest semicontinuous reflection observed in the data (red arrows in Figure 3.4.1) occurs between 0.325 and $0.430 \mathrm{~s}$, is linear and has an apparent velocity of $8990 \mathrm{~m} / \mathrm{s}$. The next event is more complex and appears to be a mixed record of at least two identifiable events: a discontinuous linear reflection (blue arrows in Figure 3.4.1) between 0.382 and $0.573 \mathrm{~s}$ with an apparent velocity of $4930 \mathrm{~m} / \mathrm{s}$ and a curved reflection (yellow arrows in Figure 3.4.1) between 0.402 and $0.501 \mathrm{~s}$ and an apparent velocity of $9560 \mathrm{~m} / \mathrm{s}$. The next reflection (purple arrows in Figure 3.4.1) is relatively continuous, linear, occurs between 0.473 and $0.625 \mathrm{~s}$ and has an apparent velocity of $6190 \mathrm{~m} / \mathrm{s}$. The last identified reflection (orange arrows in Figure 3.4.1) is curved and continuous between 0.629 and $0.760 \mathrm{~s}$ with 
an apparent velocity of $7230 \mathrm{~m} / \mathrm{s}$. This event also contains the strongest amplitudes in this data. There are several other weak or discontinuous reflections present in this data set. 


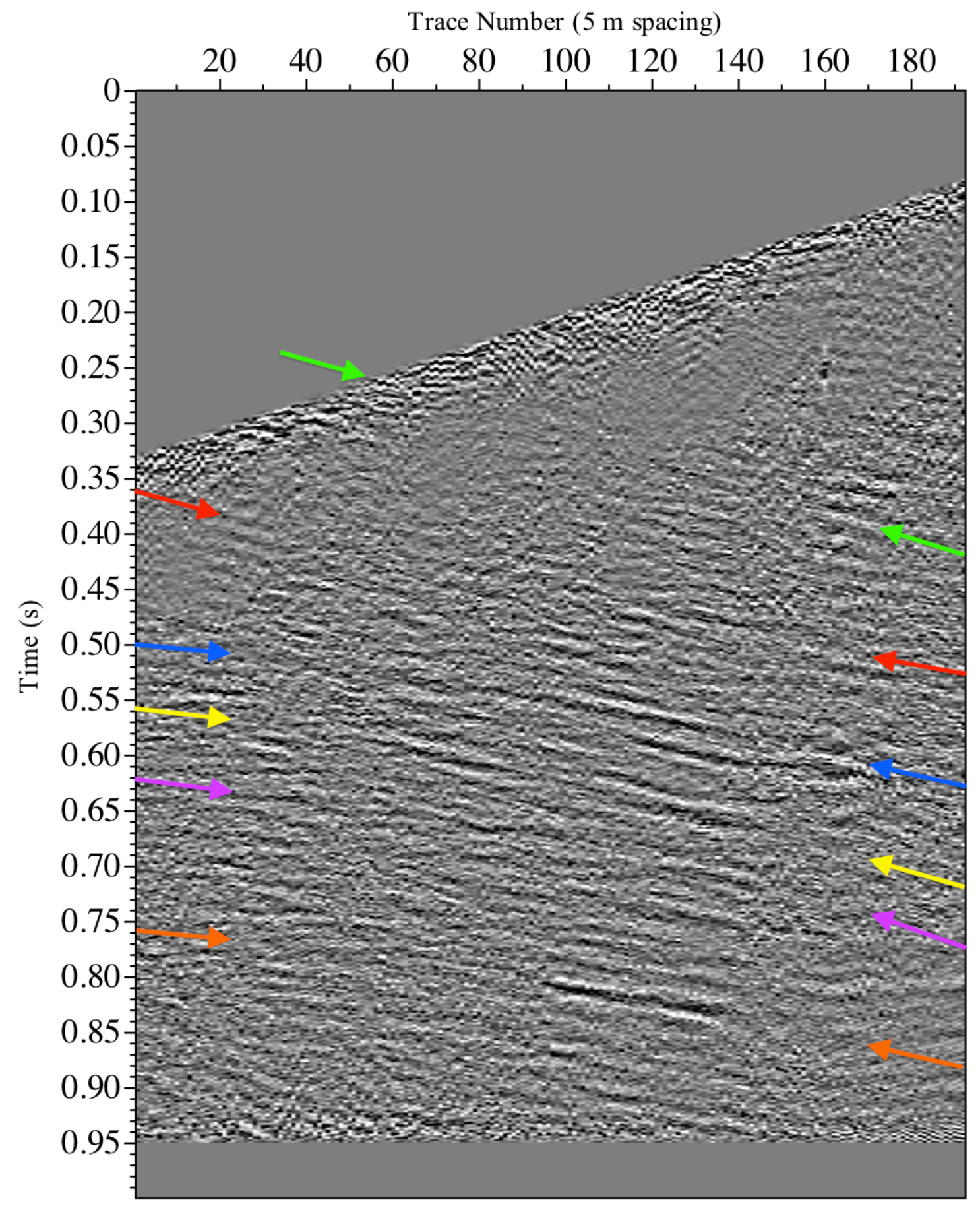

Figure 3.4.2 - Processed zero-offset dynamite source transverse component VSP data from 4Q66W3 with prominent reflections identified by coloured arrows. 
Table 3.4.2 - Summary of annotated events in the zero-offset dynamite source transverse component VSP shot gather shown in Figure 3.4.2.

\begin{tabular}{|c|c|c|c|c|c|c|c|}
\hline \begin{tabular}{|l} 
Borehole \\
Data set \\
Component \\
Figure \\
\end{tabular} & \begin{tabular}{|l} 
4Q66W3 \\
Zero-offset dynami \\
Transverse \\
3.4 .2 \\
\end{tabular} & & & & & & \\
\hline Arrow Colour & Direct / Reflected & Alignment & Continuity & $\begin{array}{c}\text { Trace } 1 \\
(\mathrm{~s})\end{array}$ & \begin{tabular}{|c|}
$\begin{array}{c}\text { Trace 192 } \\
\text { (s) }\end{array}$ \\
\end{tabular} & \begin{tabular}{|c|}
$\begin{array}{c}\text { Apparent velocity } \\
(\mathrm{m} / \mathrm{s})\end{array}$ \\
\end{tabular} & Notes \\
\hline Green & Reflection & linear & discontinuous & 0.197 & 0.417 & 4340 & intersects S-mute \\
\hline Red & Reflection & linear & semi-continuous & 0.367 & 0.530 & 5790 & \\
\hline Blue & Reflection & curved & continuous & 0.502 & 0.621 & 7870 & very strong \\
\hline Yellow & Reflection & curved & discontinuous & 0.559 & 0.701 & 6650 & \\
\hline Purple & Reflection & linear & semi-continuous & 0.624 & 0.767 & 6600 & \\
\hline Orange & Reflection & curved & discontinuous & 0.758 & 0.881 & 7670 & \\
\hline
\end{tabular}

The horizontal components of the same data set do not show as much reflectivity as the vertical component, but after processing some reflections were revealed. Since these events are less numerous, they may appear to stand out better in the shot gathers than those in the vertical component data. The transverse (with respect to the far-offset source) component data has been muted up to the direct S-waves because no reflections were observed above this horizon prior to muting. The first linear reflection (green arrows in Figure 3.4.2) occurs between 0.260 and $0.417 \mathrm{~s}$, intersects the mute window before the downgoing shear wave and has an apparent velocity of $4340 \mathrm{~m} / \mathrm{s}$. The next reflection (red arrows in Figure 3.4.2) is linear and semi-continuous, occurs between 0.367 and $0.530 \mathrm{~s}$ and has an apparent velocity of $5790 \mathrm{~m} / \mathrm{s}$. The next continuous event (blue arrows in Figure 3.4.2) is the strongest found in either of the horizontal components, is curved, occurs between 0.502 and $0.621 \mathrm{~s}$ and has an apparent velocity of $7870 \mathrm{~m} / \mathrm{s}$. The next curved discontinuous reflection (yellow arrows in Figure 3.4.2) occurs between 0.559 and $0.701 \mathrm{~s}$, and has an apparent velocity of $6650 \mathrm{~m} / \mathrm{s}$. The next linear semi-continuous reflection (purple arrows in Figure 3.4.2) occurs between 0.624 
and $0.767 \mathrm{~s}$ and has an apparent velocity of $6600 \mathrm{~m} / \mathrm{s}$. A final curved and discontinuous reflection (orange arrows in Figure 3.4.2) can be seen with an apparent velocity of 7670 $\mathrm{m} / \mathrm{s}$. Several other reflections can also be seen throughout the data set. As in the case of the vertical component data, reflections exhibit a wide range of apparent velocities. 


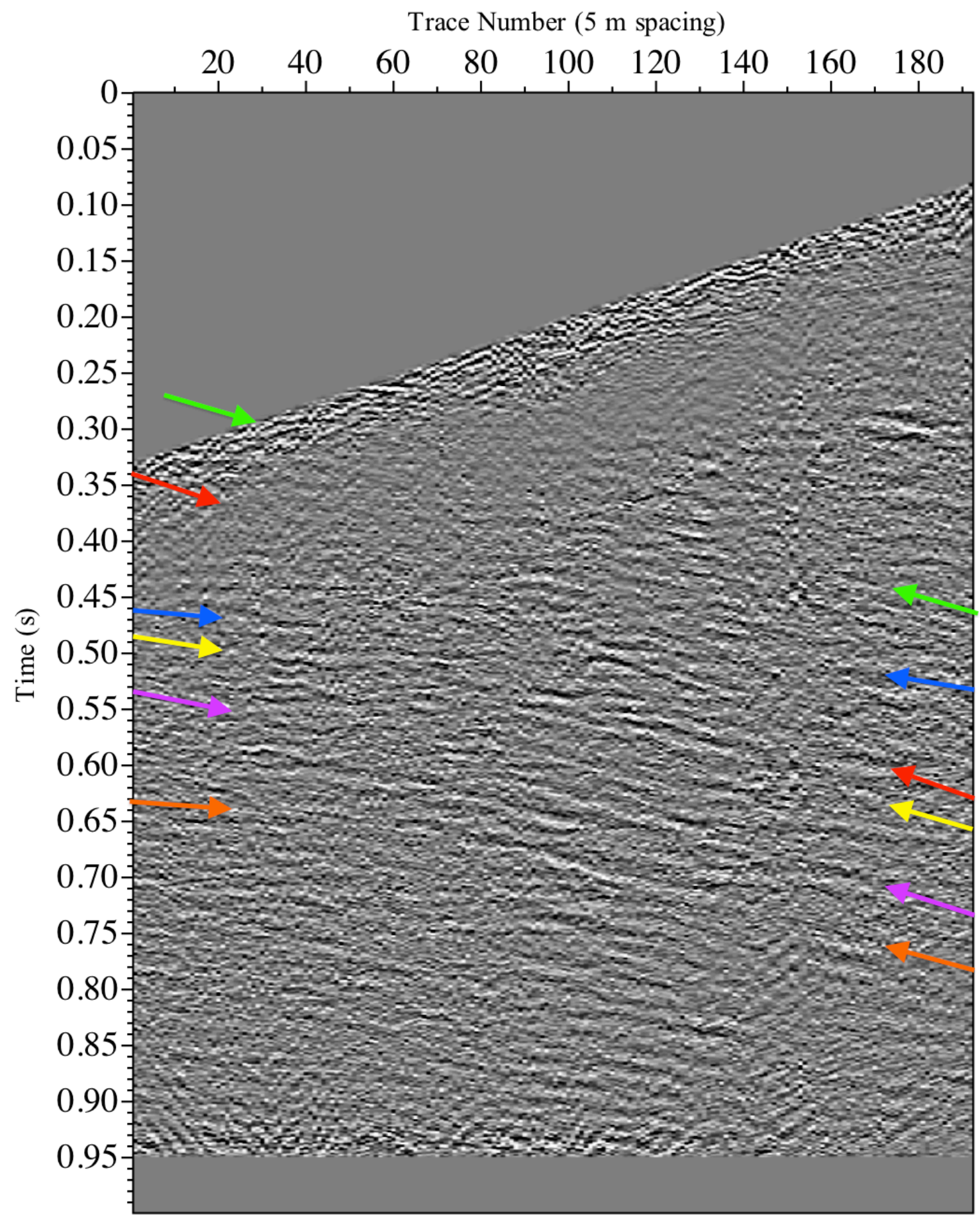

Figure 3.4.3 - Processed zero-offset dynamite source radial component VSP data from 4Q66W3 with prominent reflections identified by coloured arrows. 
Table - 3.4.3 - Summary of annotated events in the zero-offset dynamite source radial component VSP shot gather shown in Figure 3.4.3.

\begin{tabular}{|c|c|c|c|c|c|c|c|}
\hline \begin{tabular}{|l|} 
Borehole \\
Data set \\
Component \\
Figure \\
\end{tabular} & \begin{tabular}{|l} 
4Q66W3 \\
Zero-offset dynami \\
Radial \\
3.4 .3 \\
\end{tabular} & & & & & & \\
\hline Arrow Colour & Direct / Reflected & Alignment & Continuity & $\begin{array}{c}\text { Trace } 1 \\
\text { (s) }\end{array}$ & \begin{tabular}{|c|} 
Trace 192 \\
(s)
\end{tabular} & \begin{tabular}{|c|}
$\begin{array}{c}\text { Apparent velocity } \\
(\mathrm{m} / \mathrm{s})\end{array}$ \\
\end{tabular} & Notes \\
\hline Green & Reflection & linear & discontinuous & 0.254 & 0.462 & 4740 & \\
\hline Red & Reflection & linear & semi-continuous & 0.340 & 0.629 & 3250 & strong, slow \\
\hline Blue & Reflection & linear & discontinuous & 0.461 & 0.530 & 13720 & \\
\hline Yellow & Reflection & curved & semi-continuous & 0.485 & 0.656 & 5520 & \\
\hline Purple & Reflection & slightly curved & semi-continuous & 0.532 & 0.740 & 4520 & \\
\hline Orange & Reflection & curved & semi-continuous & 0.642 & 0.783 & 6650 & \\
\hline
\end{tabular}

Reflectivity in the radial (with respect to the far-offset source) component data is comparable to that of the transverse component data set. A similar window mute up to the direct S-wave was applied. The first identified reflection (green arrows in Figure 3.4.3) is discontinuous and linear, intersects the downgoing S-wave at $0.292 \mathrm{~s}$, reaches the uppermost geophone at $0.462 \mathrm{~s}$ and has an apparent velocity of $4740 \mathrm{~m} / \mathrm{s}$. The next reflection (red arrows in Figure 3.4.3) occurs between 0.340 and 0.629 s. It is linear, semi-continuous, moderately strong and is the slowest identified reflection in all of the field data, with an apparent velocity of $3250 \mathrm{~m} / \mathrm{s}$. Intersecting the previous event is a linear, discontinuous reflection (blue arrows in Figure 3.4.3) occurring between 0.461 and $0.530 \mathrm{~s}$, which has an apparent velocity of $13720 \mathrm{~m} / \mathrm{s}$. The next reflection (yellow arrows in Figure 3.4.3) is curved and semi-continuous, occurs between 0.485 and $0.656 \mathrm{~s}$, and has an apparent velocity of $5520 \mathrm{~m} / \mathrm{s}$. Shortly following this event is another slightly curved and semi-continuous reflection (purple arrows in Figure 3.4.3) occurring between 0.532 and $0.740 \mathrm{~s}$ with an apparent velocity of $4520 \mathrm{~m} / \mathrm{s}$. The final identified reflection in the radial component data (orange arrows in Figure 3.4.3) is curved and semi- 
continuous, occurs between 0.642 and $0.783 \mathrm{~s}$ and has an apparent velocity of $6650 \mathrm{~m} / \mathrm{s}$. As with the other components, several other events are noticeable but have not been annotated in Figure 3.4.3. 


\section{0 - 3D finite difference modeling}

\section{1 - Parameters}

\subsection{1 - Model assembly}

Computer simulations of the seismic response were conducted for detailed 3D geological models to allow comparison with the seismic events observed in the field VSP data, with a particular focus on reflections from the known ore lenses. The Flin Flon 3D Knowledge Cube (Figure 4.1.1.1a) was used as a starting point to build the models to be used in this study because it already contained the major geological units, large-scale structures and the known ore lenses of the Flin Flon Main, Callinan and 777 deposits. The Knowledge Cube is a collection of $3 \mathrm{D}$ vector and surface data representing geological maps, contacts, structures, borehole lithological logs, and 2D and 3D seismic data (Schetselaar et al., 2010). The approach toward model assembly for this study was to create several much smaller voxets (i.e. sets of voxels) representing specific rock types within the Knowledge Cube using gOcad software. The outer dimensions and voxel resolution were chosen based on the requirements of the modeling software and the limitations of the computer cluster to be used. A model built of smaller voxels is able to replicate higher frequency seismic waves but will have more data entries, causing longer simulation runtimes. A voxel resolution of $5 \mathrm{~m}$ x $5 \mathrm{~m}$ x $5 \mathrm{~m}$ allows frequencies of up to $180 \mathrm{~Hz}$ to be accurately simulated, thus modeling most of the downgoing and upgoing 
signal of the field VSP surveys (Figure 3.3.3.14). Also, as borehole 4Q66W3 is the focus of this study, the chosen voxet dimensions were centered around its borehole collar.

Four separate voxets were generated, each with different geological units represented to demonstrate the various reflection signatures of those units (Table 4.1.1.1). First and most simply, a 'Binary' voxet (Figure 4.1.1.1b), which contained all of the ore lenses in a background with physical properties of meta-basalt was built. This was used to simulate the timing and apparent velocities of ore lens reflections to the receiver array in 4Q66W3. A voxel resolution of $5.031 \mathrm{~m}$ (northing) x $5.037 \mathrm{~m}$ (easting) x $4.485 \mathrm{~m}$ (depth) was used with voxet outer dimensions of $2737 \mathrm{~m}$ (northing) by $1864 \mathrm{~m}$ (easting) by $1561 \mathrm{~m}$ (depth). This resulted in 70,045,440 voxels. Five versions of the 'Binary' voxet were used in simulations, one which considered all of the ore lenses together and one each considering only the Callinan North, Callinan East, Callinan South and 777 groups of ore lenses(Figure 4.1.1.2), respectively. 
Table 4.1.1.1 - Summary of the geological units and rock types represented in each of the four voxets used for modeling in this study.

\begin{tabular}{|l|l|cccc|}
\hline \multirow{2}{*}{ Geological Unit } & \multicolumn{1}{|c|}{ Rock Type } & \multicolumn{4}{c|}{ Voxet } \\
& & 'Binary' & 'Ore/Missi' 'Lithological' & 'No Ore' \\
\hline Callinan Deposit & massive sulphide ore & $\checkmark$ & $\checkmark$ & $\checkmark$ & \\
777 Deposit & massive sulphide ore & $\checkmark$ & $\checkmark$ & $\checkmark$ & $\checkmark$ \\
\hline Club Mb & meta-basalt & $\checkmark$ & $\checkmark$ & $\checkmark$ & $\checkmark$ \\
\hline Blue Lagoon Mb & meta-basalt & $\checkmark$ & $\checkmark$ & $\checkmark$ & $\checkmark$ \\
\hline Millrock Mb & meta-basalt & $\checkmark$ & $\checkmark$ & $\checkmark$ & $\checkmark$ \\
& coherent rhyolite & & & $\checkmark$ & $\checkmark$ \\
& argillite & & & $\checkmark$ & $\checkmark$ \\
\hline mafic fragmentals & & & $\checkmark$ & $\checkmark$ \\
\hline Hidden Fm & lapilli tuff & & & $\checkmark$ & $\checkmark$ \\
\hline Louis Fm & meta-basalt & $\checkmark$ & $\checkmark$ & $\checkmark$ & $\checkmark$ \\
\hline Missi Gp & meta-sandstone & & $\checkmark$ & $\checkmark$ & \\
\hline
\end{tabular}




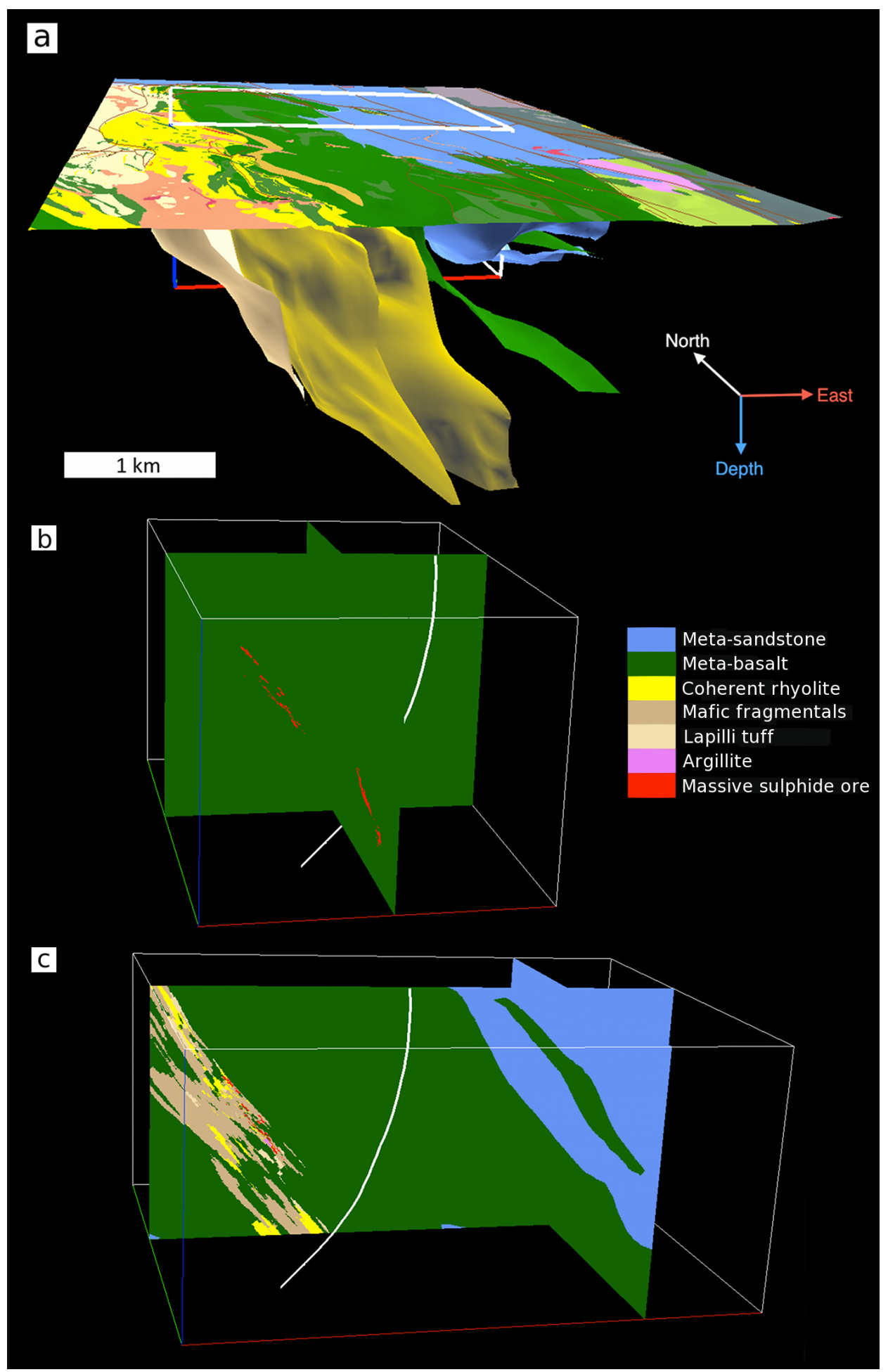

Figure 4.1.1.1 - Assembly of voxets for 3D finite difference modeling: a) lithological surface model from the Flin Flon 3D Knowledge Cube ('Lithological' voxet sub-volume is shown), b) 'Binary' voxet (4Q66W3 is shown in white), c) 'Lithological' voxet. 


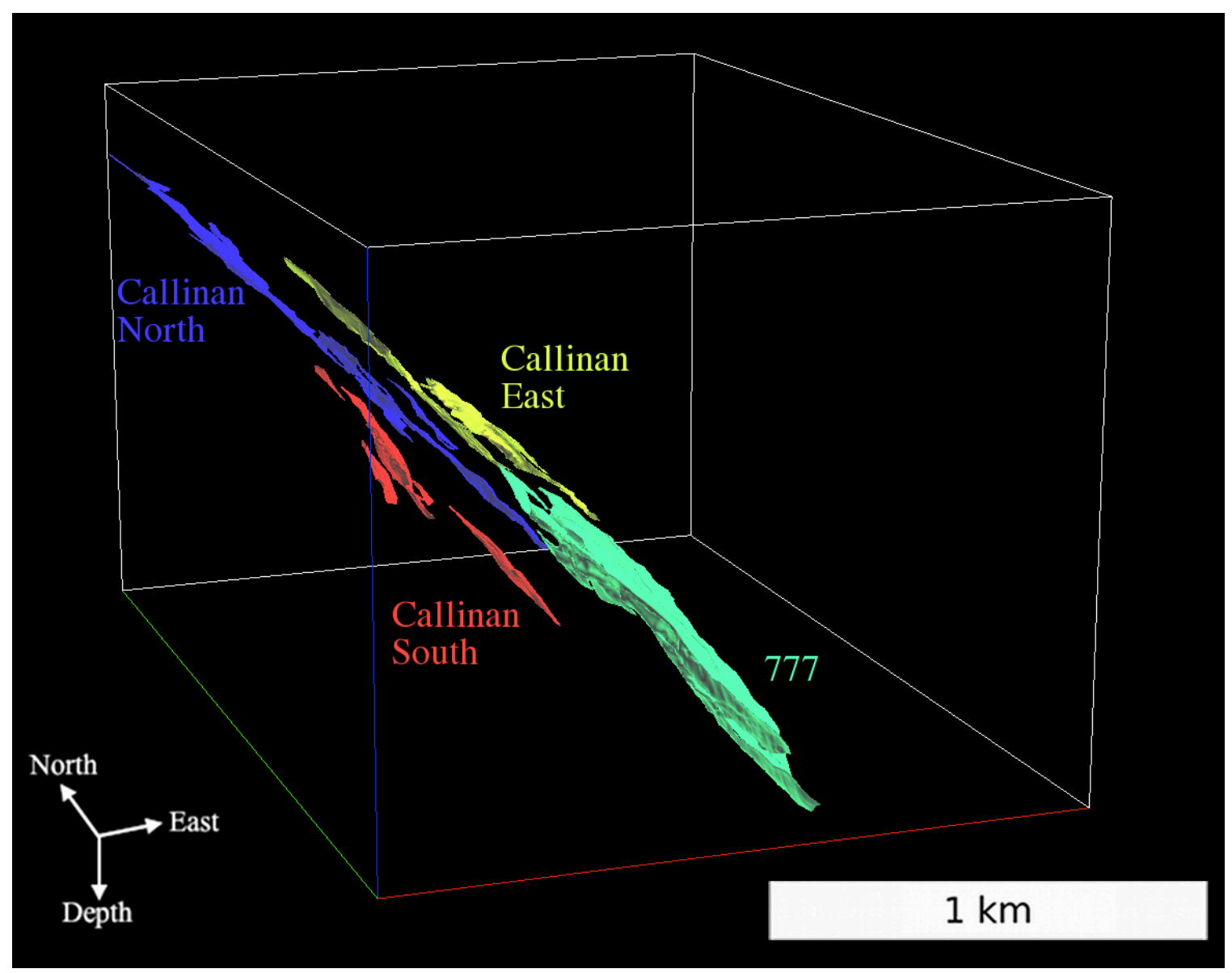

Figure 4.1.1.2 - Ore zones of the 'Binary' model, based on stratigraphic level. It should be noted that these zones are not the same as those identified by Hudbay Minerals Inc.

The next 'Ore/Missi' voxet built includes the contacts between the meta-basalt and the Missi metasedimentary basin, the nearest of which lies within $300 \mathrm{~m}$ from the collar of 4Q66W3, in order to identify any reflections originating from this contact. For this voxet the volume was extended $1410 \mathrm{~m}$ to the East to include the Ross Lake and Channing faults (D7) and a volcanic imbricate slice within the basin. This increased the voxet to $123,052,800$ voxels, which remained unchanged for the remaining two voxets.

Manipulation of many of the 3D surfaces defining the basin, faults and the volcanic imbricate slice using gOcad was required to isolate sub-volumes within the model 
defined by these features. In order to reconcile data from the existing surfaces, a borehole lithological log and two of the $2 \mathrm{D}$ seismic sections, a new thrust fault parallel to the Club Lake fault (D4) but between the Ross Lake and Channing faults was created (shown in Figure 4.1.1.3). The rock type of each voxel within the isolated sub-volumes could then be interpolated as either meta-sandstone or basalt, adding to the voxet already containing the ore lenses.

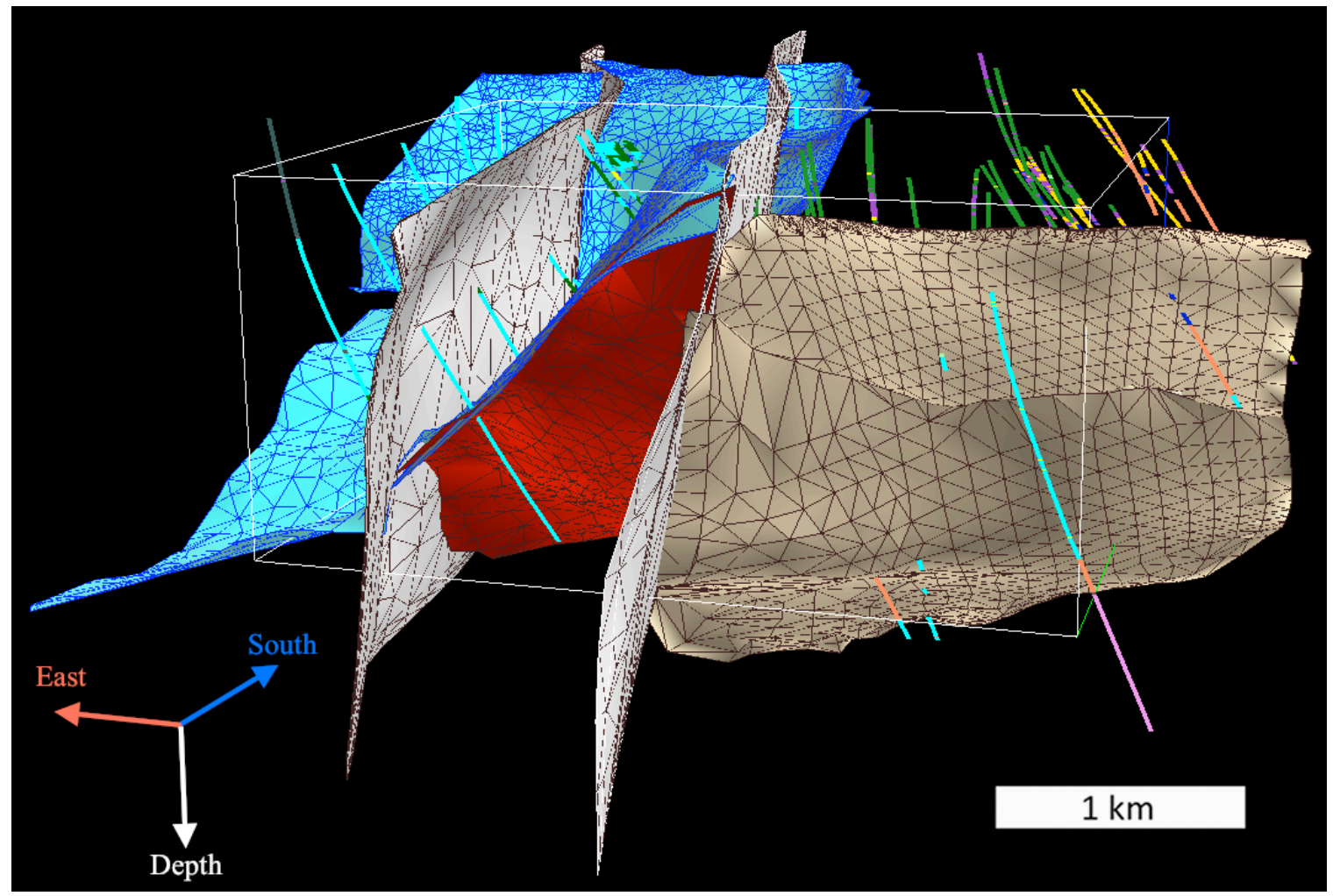

Figure 4.1.1.3 - A new thrust fault, shown in red, built in gOcad to reconcile geological

data. The Ross Lake and Channing faults are shown in grey, the Club Lake thrust fault is shown in beige and the base of the Missi basin is shown in blue. Downhole lithological logs are also displayed which were used to constrain the new thrust fault. 
The third 'Lithological' voxet (Figure 4.1.1.1c) added several other geological units contained within the 3D Knowledge Cube. Specifically, volcaniclastics, argillites and rhyolites of the Blue Lagoon and Millrock members comprising the ore horizons were included, which represents a more realistic geological scenario. Any reflections originating from these horizons would have very similar timings and apparent velocities to those of the corresponding ore lens reflections. Finally, a 'No Ore' voxet was created which contains all of the geological units in the 'Lithological' voxet but replaces all of the ore lenses found in the 'Binary' voxet with rhyolite. Simulations using this voxet should demonstrate the reflection signatures of only the host geological units. 


\subsection{2 - Pre-processing}

Each gOcad voxet was exported in SEGY format and pre-processed to prepare the model for simulations with the 3D finite difference modeling software. A series of operations needed to be performed to convert, resort, rearrange and add physical properties to the voxets, beginning with conversion from SEGY to Seismic Unix .su format. Then, the data were flipped about a horizontal axis and resorted such that traces are read in the required $3 \mathrm{D}$ geometric order (i.e. from height above voxet bottom, northing, easting to depth below voxet top, easting, northing). Then, the voxets were converted to an ASCII file to import to MATLAB and add physical properties to each voxel with custom scripts. Each of the voxets were divided into three physical property model files and exported in 4-bit native float binary format.

\subsection{3 - Physical properties}

For each of the 7 rock types included in the various models, density, P-wave velocity and S-wave velocity values needed to be specified for finite difference modeling (Table 4.1.3.1). These values for each rock type were kept constant for all of the models, and a consistent means of determining them had to be found. During and after the 2006 VSP survey, downhole geophysical logs were acquired by the GSC and DGI Geoscience Inc. in several of the boreholes around Flin Flon including 4Q66W3. Also, representative core samples from some boreholes were sent to the GSC's High Pressure Laboratory 
(HPL) at Dalhousie University in Halifax, Nova Scotia, Canada for laboratory testing.

Determining the most representative values to use required careful consideration and examination of all the available data.

Table 4.1.3.1 - Summary of physical rock properties applied to the voxets in MATLAB for 3D finite difference modeling. 'HPL Core' indicates measurements from core taken at the GSC's High Pressure Laboratory. Other downhole density and P-wave velocity measurements indicate which organization and borehole the measurement came from. All S-wave velocities were calculated using a $\mathrm{Vp} / \mathrm{Vs}$ ratio taken from the HPL core measurements.

\begin{tabular}{|l|c|l|c|l|c|c|c|c|}
\hline \multicolumn{1}{|c|}{ Rock Type } & $\begin{array}{c}\text { Density } \\
\left(\mathbf{k g} / \mathbf{m}^{3}\right)\end{array}$ & From & $\begin{array}{c}\text { P-Wave } \\
\text { Velocity } \\
(\mathbf{m} / \mathbf{s})\end{array}$ & From & $\begin{array}{c}\text { P-wave } \\
\text { Impedance } \\
\left(\mathbf{k g} / \mathbf{m}^{2} \mathbf{s}\right)\end{array}$ & $\begin{array}{c}\text { S-Wave } \\
\text { Velocity } \\
(\mathbf{m} / \mathbf{s})\end{array}$ & From & $\begin{array}{c}\text { S-wave } \\
\text { Impedance } \\
\left(\mathbf{k g} / \mathbf{m}^{2} \mathbf{s}\right)\end{array}$ \\
\hline coherent rhyolite & 2740 & HPL Core & 6008.9 & GSC 4Q66 & $1.65 \mathrm{E}+07$ & 3609.2 & Core $\mathrm{Vp} / \mathrm{Vs}$ & $9.89 \mathrm{E}+06$ \\
mafic fragmentals & 2824 & GSC 4Q66 & 6013.7 & GSC 4Q66 & $1.70 \mathrm{E}+07$ & 3612.1 & Core $\mathrm{Vp} / \mathrm{Vs}$ & $1.02 \mathrm{E}+07$ \\
lapilli tuff & 2865 & HPL Core & 6031.9 & GSC 4Q66 & $1.73 \mathrm{E}+07$ & 3623.1 & Core $\mathrm{Vp} / \mathrm{Vs}$ & $1.04 \mathrm{E}+07$ \\
argillite & 2830 & HPL Core & 6180.8 & GSC 4Q66 & $1.75 \mathrm{E}+07$ & 3712.5 & Core $\mathrm{Vp} / \mathrm{Vs}$ & $1.05 \mathrm{E}+07$ \\
massive sulphide ore & 4360 & HPL Core & 6117.9 & DGI T7X074 & $2.67 \mathrm{E}+07$ & 3674.7 & Core $\mathrm{Vp} / \mathrm{Vs}$ & $1.60 \mathrm{E}+07$ \\
meta-basalt & 2955 & HPL Core & 6058.3 & GSC 4Q66 & $1.79 \mathrm{E}+07$ & 3638.9 & Core $\mathrm{Vp} / \mathrm{Vs}$ & $1.08 \mathrm{E}+07$ \\
meta-sandstone & 2745 & HPL Core & 5696.5 & DGI 4Q83 & $1.56 \mathrm{E}+07$ & 3421.6 & Core $\mathrm{Vp} / \mathrm{Vs}$ & $9.39 \mathrm{E}+06$ \\
\hline
\end{tabular}

Direct laboratory measurement at atmospheric pressures of the core samples from 4Q66 and other boreholes in Flin Flon was decided to be the most accurate method to determine densities. The density value for mafic fragmentals was taken as the mean of the GSC downhole log of 4Q66.

One of the major assumptions for this study is that the ore lenses, as with all rock units, are homogeneous in physical properties. However, much of the Callinan deposit was mined out prior to the field VSP survey, while most of the 777 deposit remained intact. 
The mined out lenses were backfilled with a low acoustic impedance material, which is expected to produce as strong a reflectivity with the meta-basalt as the intact ore (White et al., 2012, Malinowski et al., 2012). Also, strong variability in the mineralogy, texture and therefore, acoustic properties of the intact ore zones is known (Tessier and O'Donnell, 2001). Neither of these factors would significantly effect the timing, shape or apparent velocity of observed reflections. Thus, the only differences expected in the field data would be a more complex response and an opposite polarity of reflected waves from the mined out lenses when compared to the modeled VSP data.

From the field VSP data from 4Q66W3, the average downgoing P-wave and S-wave velocities were measured to be $6150 \mathrm{~m} / \mathrm{s}$ and $3860 \mathrm{~m} / \mathrm{s}$, respectively, which provided an estimate of the values to expect for meta-basalt. It is noted that since 4Q66W3 is deviated these values should be slightly overestimated. P-wave velocities for all rock types were taken from averages of the GSC and DGI downhole logs of boreholes 4Q66, T7X074 and 4Q83. None of the data sets provided a consistent set of S-wave velocities, so an average ratio between the $\mathrm{P}$ - and S-wave velocities was found from the laboratory core measurements and applied. 


\subsection{4 - 3D finite difference modeling using SOFI3D}

The modeling software used in this study was SOFI3D, a 3D viscoelastic finitedifference seismic modeling program developed at the Karlsruhe Institute of Technology. SOFI3D offers the advantages of considering viscoelastic propagation effects (i.e. attenuation and dispersion), employing higher-order finite-difference operators, applying perfectly-matched layer boundary conditions at the edges of the numerical mesh and works in a message-passing interface (MPI) parallel environment (Bohlen, 2002). Using MPI, models are subdivided into evenly-sized volumes to be handled by designated processing elements (i.e groups of processors). Viscoelastic attenuation effects were not considered in this study because seismic quality factors were not measured for these rocks.

SOFI3D models seismic waves by approximating solutions of the wave equation using finite-difference operators along with particle velocities, stresses and Lamé parameters calculated at discrete cartesian coordinates and times (Bohlen, 2002). Increasing the order of the spatial finite-difference operator increases the number of weighted approximations between each spatial interval and therefore considers higher frequency waves but will also require more computations to take place. The order of the spatial finite-difference equation and the set of coefficients for each approximation are userdefined, based on available computational resources and the desired frequency range. 
Two important considerations must be made to ensure a stable simulation. The spatial sampling interval must be less than or equal to $1 / 2$ the minimum wavelength (known as the Nyquist criterion) and the temporal sampling interval must not exceed the time for a wave to travel between two adjacent grid points (known as the Courant-Freidrichs-Lewy criterion). These two criteria define the spatial and temporal sample intervals, the minimum allowable S-wave velocity, the maximum allowable P-wave velocity and the maximum wave frequency, which can be simulated (Bohlen, 2002). With all of the above considerations and the model volume in mind, 2nd-order temporal and 8th-order Holberg spatial finite-difference operators, a temporal sample period of $0.0002 \mathrm{~s}$ and a spatial resolution of $5 \mathrm{~m}$ were chosen for this study (Table 4.1.4.1). These parameters would simulate frequencies up to $180 \mathrm{~Hz}$ with the required maximum and minimum velocities $(6180.8 \mathrm{~m} / \mathrm{s}$ and $3421.6 \mathrm{~m} / \mathrm{s}$, respectively) for the rock types considered.

Table 4.1.4.1 - Parameters use for 3D finite difference modeling in this study.

\begin{tabular}{|c|c|c|c|c|}
\hline Software & \multicolumn{4}{|c|}{ SOFI3D finite difference } \\
\hline FD Coefficients & \multirow{3}{*}{\multicolumn{4}{|c|}{$\begin{array}{l}\text { Holberg, } 8 \text { th order spatial operator, } 2 \text { nd order temporal operator } \\
120 \text { processors, NRCAN 3D Imaging and Earth Modeling } \\
\text { Linux, mpich-1.2.7p1 }\end{array}$}} \\
\hline Computer Cluster & & & & \\
\hline Operating System, MPI interface & & & & \\
\hline Model & 'Binary' & 'Ore/Missi' & 'Lithological' & 'No Ore \\
\hline Dimensions in Voxels (East, North, Depth) & $370,544,348$ & \multicolumn{3}{|c|}{$650,544,348$} \\
\hline Voxel Resolution (East, North, Depth) & \multirow{2}{*}{\multicolumn{4}{|c|}{$\begin{array}{l}5.037 \mathrm{~m}, 5.031 \mathrm{~m}, 4.485 \mathrm{~m} \\
\text { 190. Particle Velocity (x,y z), Pressure, Curl. Divergence }\end{array}$}} \\
\hline Number of Receivers, Recorded Particle Motions & & & & \\
\hline Wavefield Propagation Time & \multicolumn{4}{|c|}{$1 \mathrm{~s}$} \\
\hline Timesteps, Sample Period & \multicolumn{4}{|c|}{$5000,0.0002 \mathrm{~s}$} \\
\hline Source Signal & \multirow{3}{*}{\multicolumn{4}{|c|}{$\begin{array}{l}\text { Ricker wavelet, point source, } 90 \mathrm{~Hz} \text { center frequency } \\
\text { Perfectly-matched layers, } 20 \text { voxels, } 8 \% \text { decay per voxel } \\
\text { VSP Shot-gathers 3D Wavefield snapshots, Logs }\end{array}$}} \\
\hline Absorbing Boundary & & & & \\
\hline Outputs & & & & \\
\hline
\end{tabular}

SOFI3D creates a local model grid based on the input parameters. Local coordinates had to be predetermined for the source and for the receiver array and input as separate ASCII 
files. The known geometry of borehole 4Q66W3 and the wireline depths of receivers allowed interpolation of receiver coordinates. These and the source location were then converted to UTM coordinates and translated to local grid coordinates. The wavefield source was defined as a point-source (i.e. explosive) Ricker wavelet with a $90 \mathrm{~Hz}$ center frequency to accommodate the $180 \mathrm{~Hz}$ frequency limit. Finally, boundary conditions must be applied to attenuate seismic waves at the modeled volume boundaries. SOFI3D uses a perfectly-matched layers method to exponentially decay plane wave solutions within the user-defined absorbing boundary frame (Komatitsch and Martin, 2007). This method was employed with a 20 voxel thick absorbing boundary and an 8 percent amplitude decay at each voxel, as recommended in Bohlen et al. (2011).

\subsection{5 - Post-processing and Outputs}

The two types of visual outputs created during a SOFI3D simulation are seismic shot gathers and 3D wavefield visualizations. Each receiver location is capable of computing divergence, rotation (i.e. curl), pressure and 3-component particle velocities at each time step of the simulation. Pressure data was not used in this study. Divergence and rotation were used to distinguish P- and S-waves, and particle velocities to simulate field VSP records. The shot gathers are saved in a user-defined seismic data format (including Seismic Unix .su, as used in this study) and 3D wavefield visualizations as binary files at each time step. 
Some minor post-processing using Seismic Unix was required for the shot gathers to be displayed and saved for comparison with other data. First, .su files from each processing element sub-volume containing receivers had to be concatenated and sorted. Then, a .su format conversion and trace header correction took place. Finally, the shot gathers were scaled, flipped about a vertical axis and either output to a monitor via X11 or exported as a postscript image (Figure 4.1.5.1). 


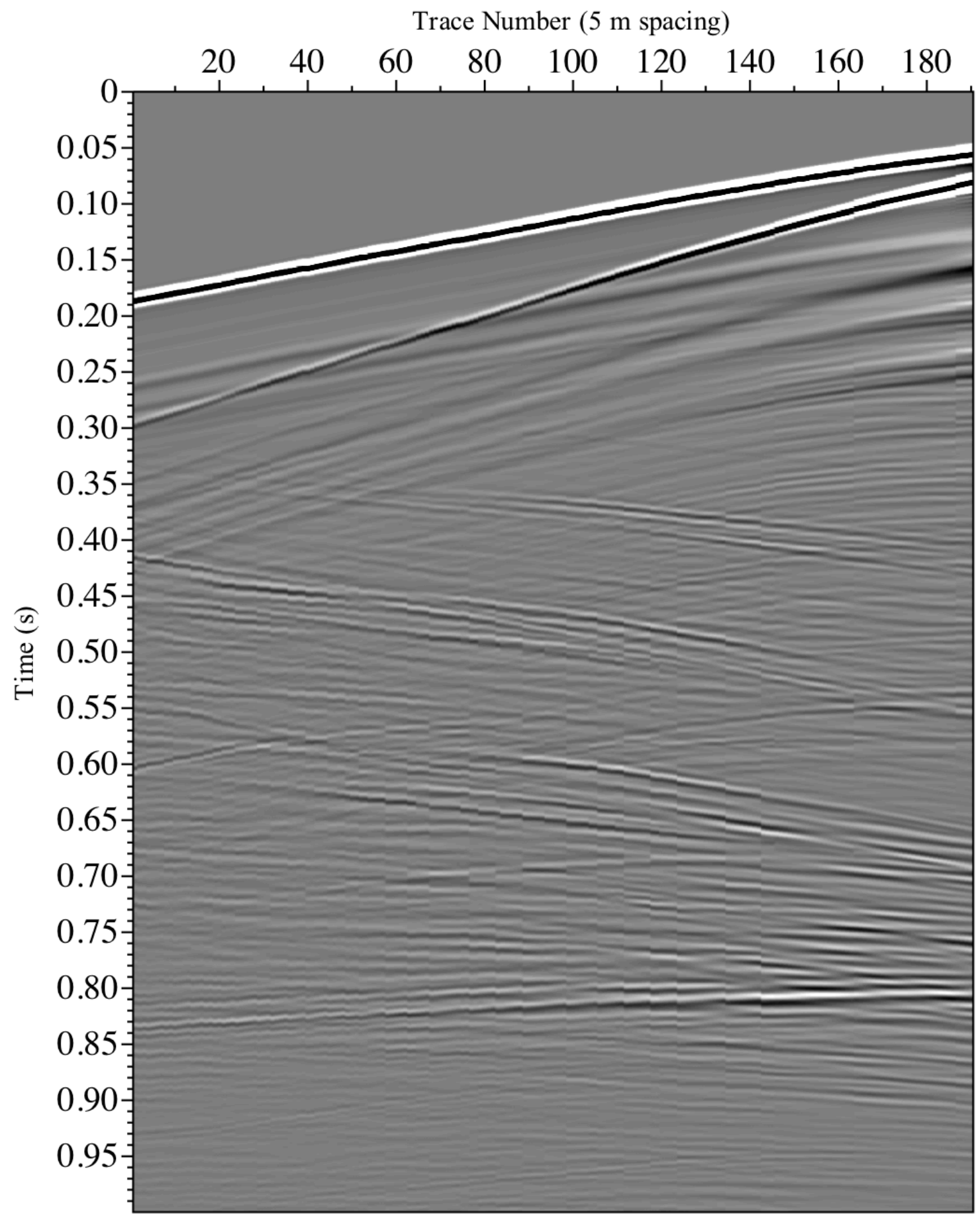

Figure 4.1.5.1 - Zero-offset vertical particle velocity synthetic VSP shot gather from borehole 4Q66W3 in the 'Lithological' model. 
The 3D wavefield visualizations also required post-processing. Only divergence and rotation data were recorded for these visualizations. First, the binary files for each time step needed to be merged into a single $4 \mathrm{D}$ binary file. These files were greater than 48 gigabytes, too large to continue processing, so a custom resampling script was written to decimate the model resolution by 2 in each spatial dimension. Then, another script was used to convert the resampled binary files into SILO format, so they could be imported and displayed using VisIt visualization software (Figure 4.1.5.2). This post-processing sequence was also applied to an acoustic impedance binary file, created by multiplying the input P-wave velocity and density binary files, on which the wavefield visualizations could be overlain for display (Figure 4.5.1.3). 


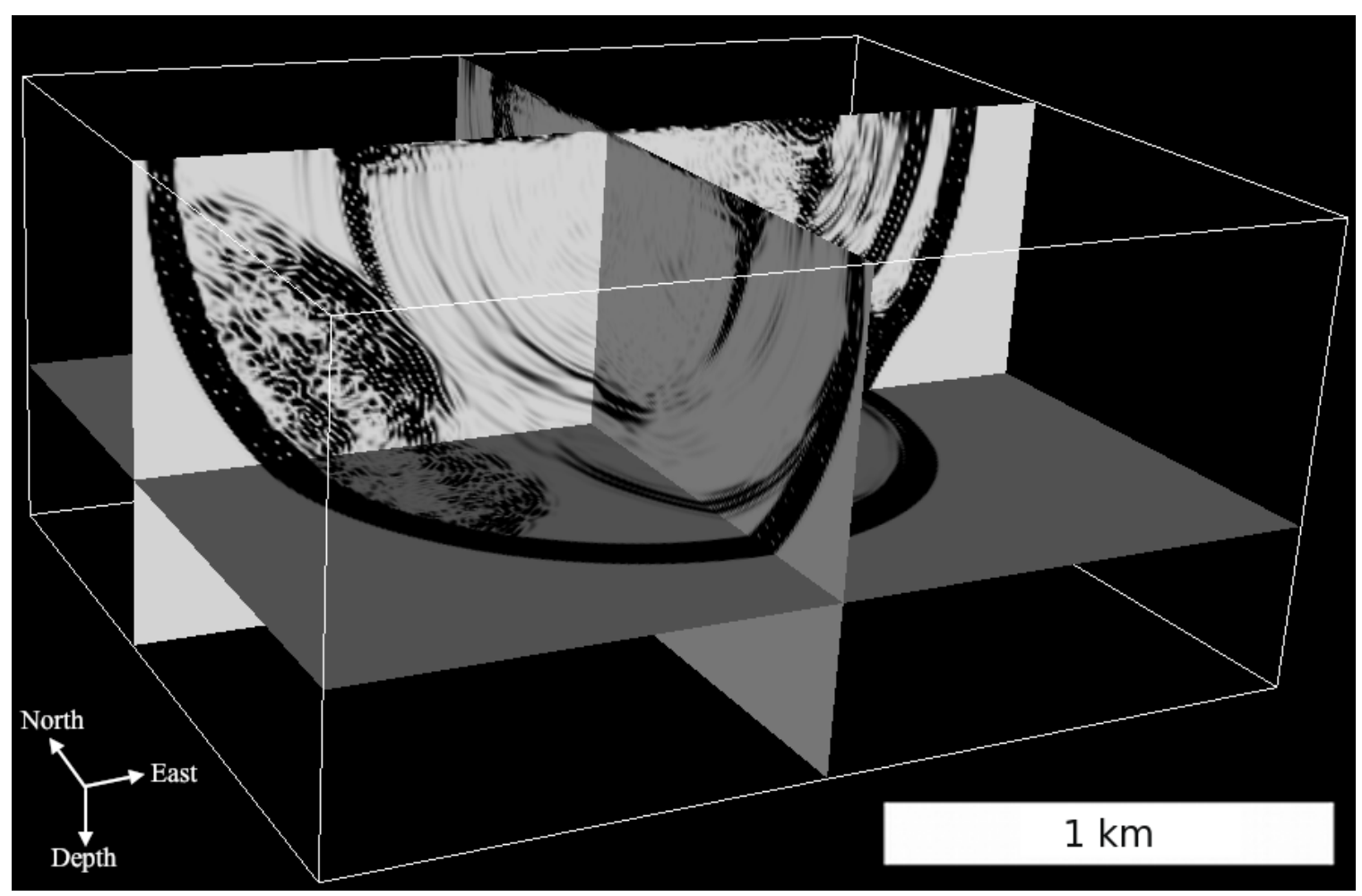

Figure 4.1.5.2 - 3D divergence wavefield visualization from the 'Lithological' model at

$0.260 \mathrm{~s}$. The source is at the intersection of the vertical planes and the surface. The location of the receiver array is approximated by the vertical intersection of the two vertical planes above the horizontal plane. 


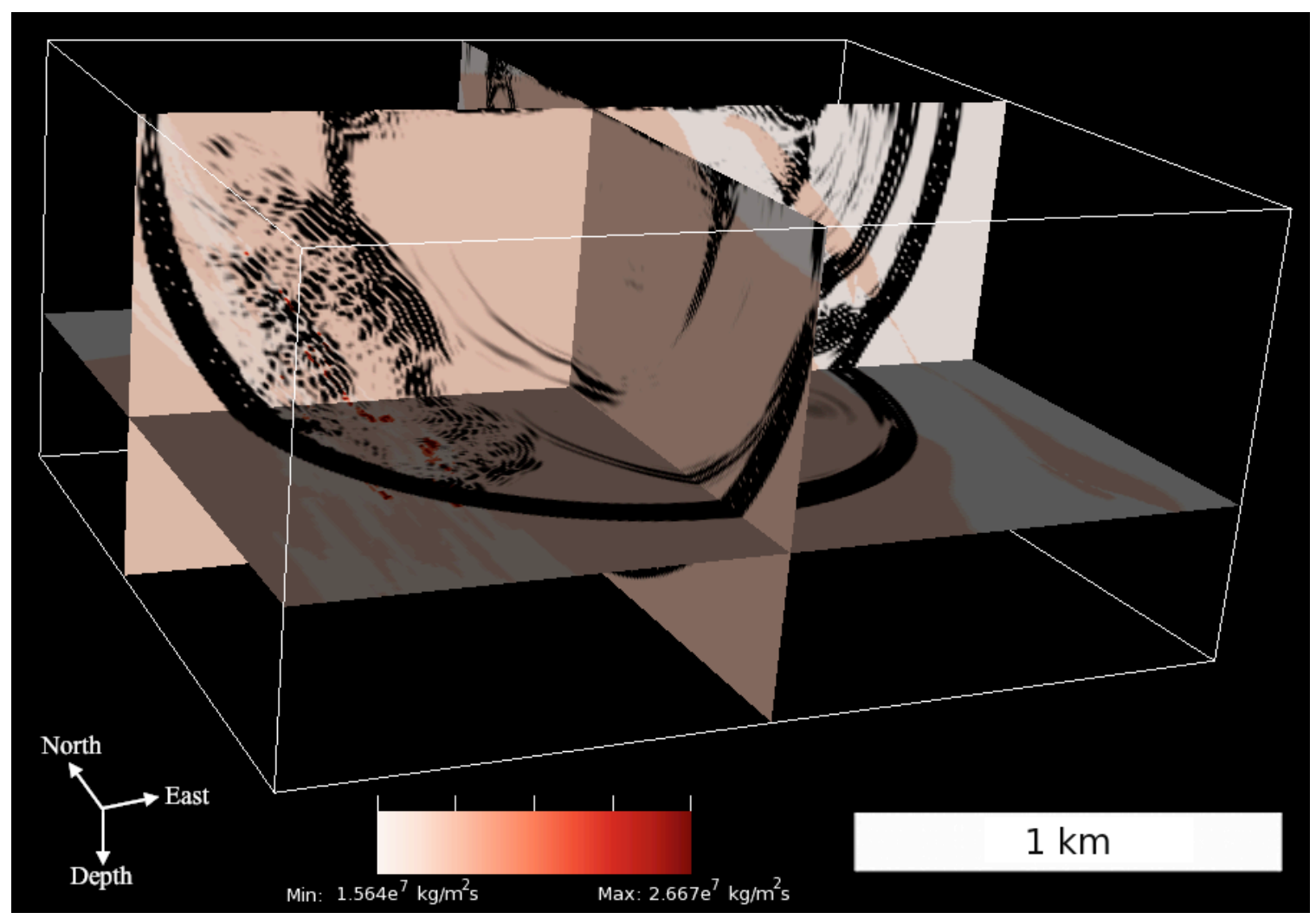

Figure 4.1.5.3 - 3D divergence wavefield visualization overlain on acoustic impedance

from the 'Lithological' model at $0.260 \mathrm{~s}$. Darker colours indicate voxels with higher acoustic impedance. 


\section{2 - 'Binary' model observations}

Shot gather and 3D wavefield visualization outputs from each of the VSP simulations in this study are displayed and discussed here. Each output records the modeled direct waves and reflections, which originate at boundaries with contrasting acoustic impedance within the model. Modeled shot gathers are directly comparable with the field shot gathers since the acquisition geometry and physical properties have been accurately replicated, demonstrated by the close match in timing and apparent velocity of the direct P- and S-waves. Because SOFI3D is able to calculate divergence, rotation and particle velocity components of the wavefield at each receiver, P-P, P-S, S-S or S-P reflections with any apparent velocity can be identified in the $t-x$ domain. Apparent velocities of all annotated direct and reflected waves have been measured linearly using the known offsets and times. In the modeled volume, 4Q66W3 does not intersect any changes in lithology so all observed reflections display an apparent velocity, controlled by the 3D geometry of the reflector and the receiver array.

An 'empty' model test was performed to detect any artifacts, which would be present in the rest of the modeling results. A very faint but identifiable artifact was seen in the resulting shot gathers (Figure 4.2.1), which will be dismissed in the results from all other models. In the 'Binary' model, the only reflectors are the ore lenses of the Callinan and 777 deposits. Divergence, rotation and 3-component particle velocity shot gathers are 
displayed and discussed below. These results should approximate the timing and apparent velocities of reflections from the ore lenses in the field VSP data. 


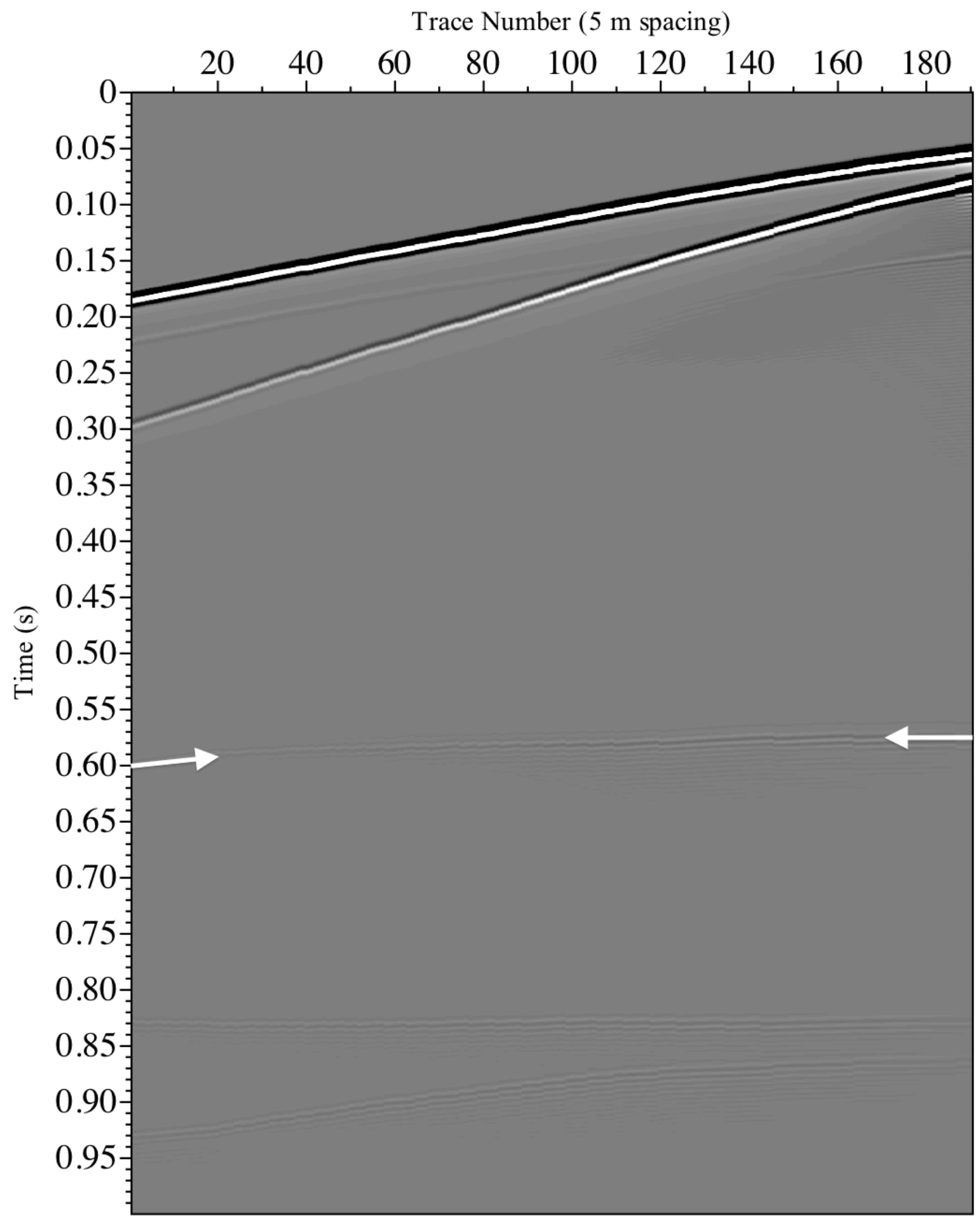

Figure 4.2.1 - Vertical particle velocity synthetic VSP shot gather from 4Q66W3 in the 'empty' test model. An example of weak but identifiable artifacts is annotated with white arrows. 
In the divergence data (Table 4.2.1 and Figure 4.2.2) the direct P-wave is much stronger than the direct S-wave and the converse is true in the rotation data (Table 4.2.2 and Figure 4.2.3). All of the reflections must be either P-P or S-P in the divergence data and either P-S or S-S in the rotation data, based on their respective particle motions. This is also shown by the apparent velocities of reflections in each, with most reflections in the divergence data being considerably faster than those in the rotation data.

In the divergence data set, the annotated reflections have relatively consistent apparent velocity (Table 4.2.1). The strongest reflections (green and yellow arrows in Figure 4.2.2) have similar travel times and occur early, between 0.327 and $0.423 \mathrm{~s}$. There are two particularly strong reflections (yellow and orange arrows in Figure 4.2.3) and the previously-mentioned image artifact is present (white arrows in Figure 4.2.3). 


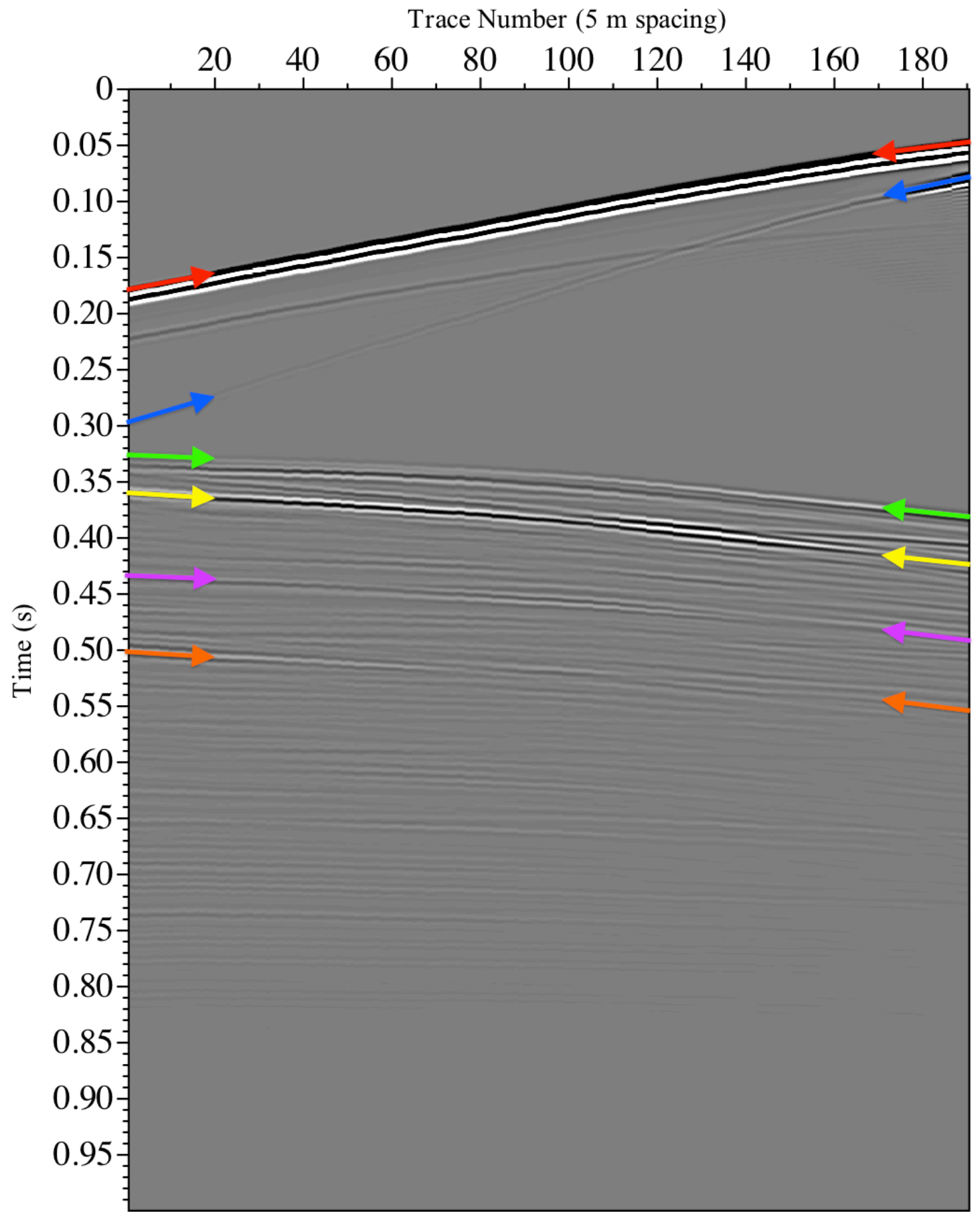

Figure 4.2.2 - Zero-offset divergence synthetic VSP shot-gather from 4Q66W3 in the 'Binary' model with prominent reflections identified by coloured arrows. 
Table 4.2.1 - Summary of annotated events in the divergence shot gather from the 'Binary' model shown in Figure 4.2.2.

\begin{tabular}{|l|l|l|l|c|c|c|c|}
\hline $\begin{array}{l}\text { Model } \\
\text { Data set } \\
\text { Figure }\end{array}$ & $\begin{array}{l}\text { 'Binary' } \\
\text { Divergence } \\
4.2 .2\end{array}$ \\
\hline Arrow Colour & Direct / Reflected & Alignment & Continuity & $\begin{array}{c}\text { Trace 1 } \\
(\mathbf{s})\end{array}$ & $\begin{array}{c}\text { Trace 190 } \\
(\mathbf{s})\end{array}$ & $\begin{array}{c}\text { Apparent velocity } \\
(\mathbf{m} / \mathbf{s})\end{array}$ & Notes \\
\hline Red & Direct P-wave & slightly curved & continuous & 0.180 & 0.042 & -6180 & very strong \\
\hline Blue & Direct S-wave & curved & continuous & 0.298 & 0.074 & -3800 & barely noticeable \\
\hline Green & Reflection & slightly curved & continuous & 0.327 & 0.381 & 15780 & \\
\hline Yellow & Reflection & curved & continuous & 0.350 & 0.423 & 11670 & \\
\hline Purple & Reflection & curved & continuous & 0.433 & 0.491 & 14690 & \\
\hline Orange & Reflection & slightly curved & mixed, $\geq 2$ & 0.501 & 0.555 & 15780 & \\
\hline
\end{tabular}




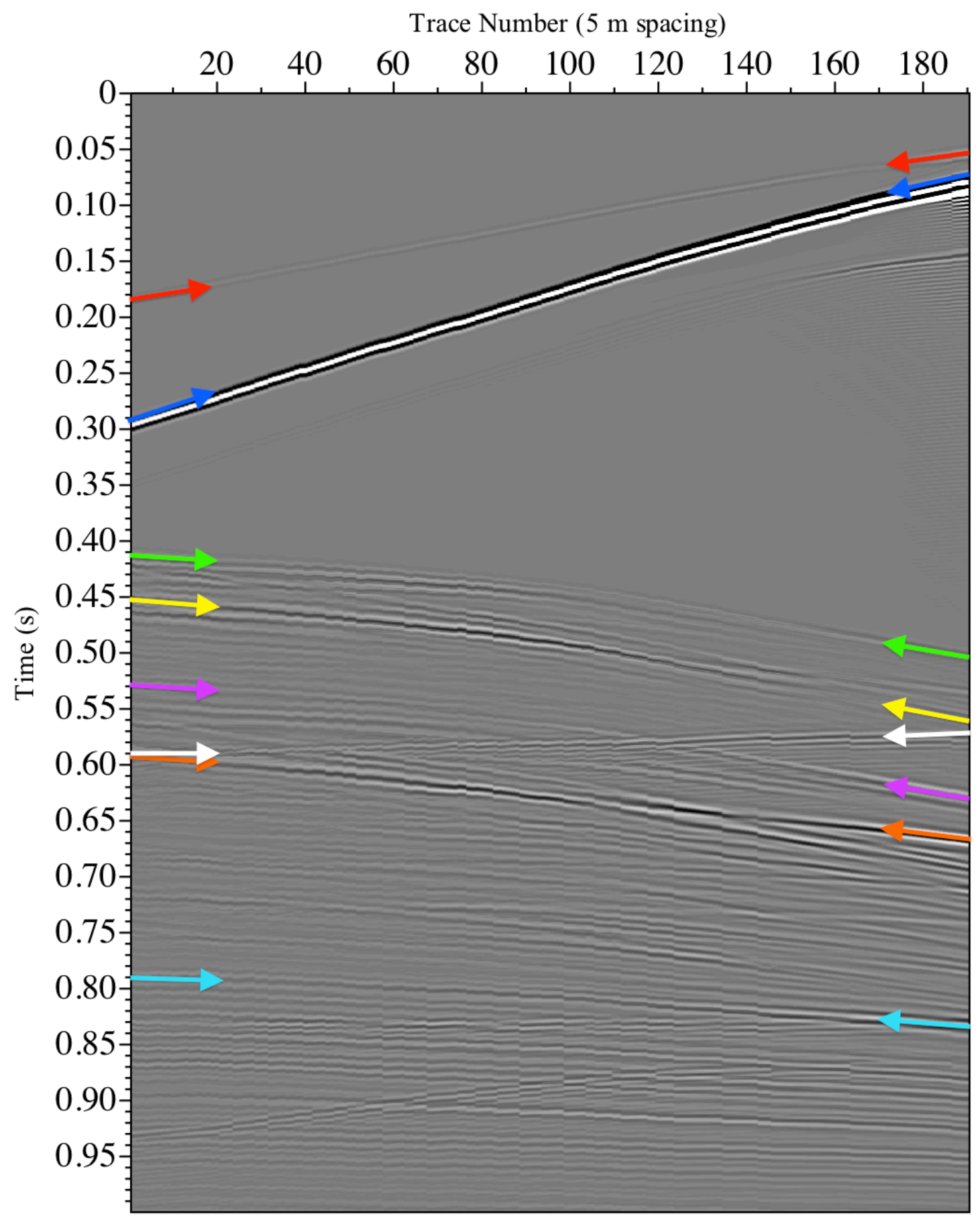

Figure 4.2.3 - Zero-offset rotation synthetic VSP shot-gather from 4Q66W3 in the

'Binary' model with prominent reflections identified by coloured arrows. 
Table 4.2.2 - Summary of annotated events in the rotation shot gather from the 'Binary' model shown in Figure 4.2.3.

\begin{tabular}{|c|c|c|c|c|c|c|c|}
\hline \begin{tabular}{|l} 
Model \\
Data set \\
Figure
\end{tabular} & $\begin{array}{l}\text { 'Binary' } \\
\text { Rotation } \\
4.2 .3\end{array}$ & & & & & & \\
\hline Arrow Colour & Direct / Reflected & Alignment & Continuity & $\begin{array}{c}\text { Trace } 1 \\
\text { (s) }\end{array}$ & $\begin{array}{l}\text { Trace } 190 \\
\text { (s) }\end{array}$ & $\begin{array}{c}\text { Apparent velocity } \\
(\mathrm{m} / \mathrm{s})\end{array}$ & Notes \\
\hline Red & Direct P-wave & slightly curved & continuous & 0.180 & 0.042 & -6180 & barely noticeable \\
\hline Blue & Direct S-wave & curved & continuous & 0.298 & 0.074 & -3800 & very strong \\
\hline Green & Reflection & curved & continuous & 0.411 & 0.508 & 8790 & \\
\hline Yellow & Reflection & curved & continuous & 0.451 & 0.562 & 7680 & \\
\hline Purple & Reflection & curved & continuous & 0.528 & 0.632 & 8190 & artifact interuption \\
\hline White & Artifact & linear & continuous & 0.598 & 0.568 & -28410 & \\
\hline Orange & Reflection & curved & mixed, 2 & 0.591 & 0.669 & 10930 & \\
\hline Light blue & Reflection & linear & continuous & 0.790 & 0.835 & 18940 & \\
\hline
\end{tabular}

All of the particle velocity shot gathers show a mix of events already seen in the divergence and rotational shot gathers, since particle velocity can record both P- and Swaves (i.e. P-P, P-S, S-S or S-P reflections are potentially captured).

In the easting particle velocity shot gather (Table 4.2.3 and Figure 4.2.4) the direct P- and S-waves appear mostly similar to the previous shot gathers (e.g. Figures 4.2.2 and 4.2.3) but a polarity reversal can be seen in both at trace 137 , not observed in any of the reflections. This effect is due to the deviated geometry of the borehole and slight offset of the source. Some of the events also appear to be mixed or overlapping reflections. The northing particle velocity shot gather (Table 4.2.4 and Figure 4.2.5) does not show the polarity reversal seen in the direct waves of the easting particle velocity shot gather. Several of the same reflections are seen in both of these horizontal components with varying apparent velocities. Several other weaker events can be seen throughout and later in both data sets. 


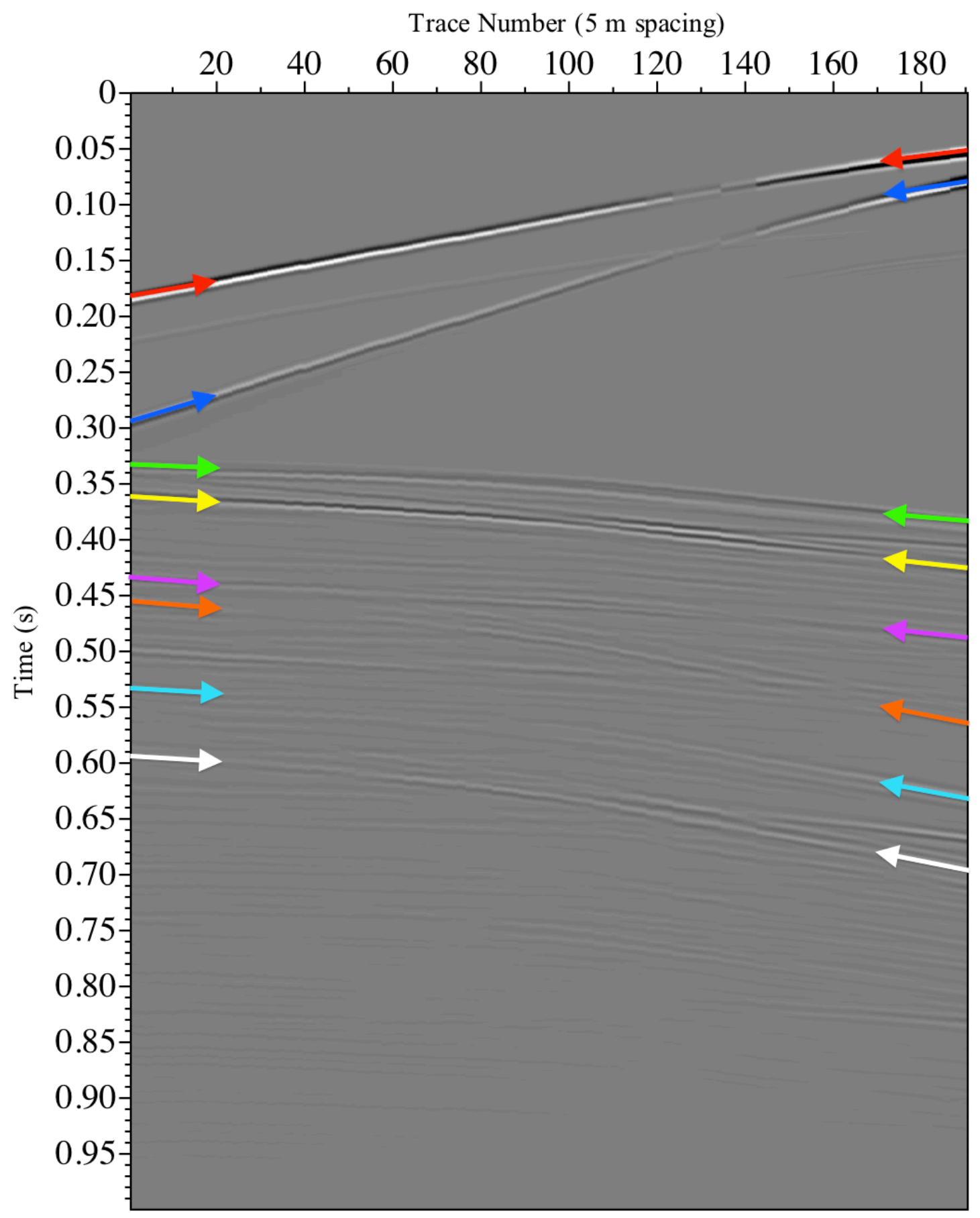

Figure 4.2.4 - Zero-offset easting particle velocity synthetic VSP shot-gather from 4Q66W3 in the 'Binary' model with prominent reflections identified by coloured arrows. 
Table 4.2.3 - Summary of annotated events in the easting particle velocity shot gather from the 'Binary' model shown in Figure 4.2.4.

\begin{tabular}{|l|l|l|l|c|c|c|l|}
\hline $\begin{array}{l}\text { Model } \\
\text { Data set } \\
\text { Figure }\end{array}$ & $\begin{array}{l}\text { 'Binary' } \\
\text { Easting particle velocity } \\
4.2 .4\end{array}$ \\
\hline Arrow Colour & Direct / Reflected & Alignment & Continuity & $\begin{array}{c}\text { Trace 1 } \\
(\mathbf{s})\end{array}$ & $\begin{array}{c}\text { Trace 190 } \\
(\mathbf{s})\end{array}$ & $\begin{array}{c}\text { Apparent velocity } \\
(\mathbf{m} / \mathbf{s})\end{array}$ & Notes \\
\hline Red & Direct P-wave & slightly curved & continuous & 0.180 & 0.042 & -6180 & Polarity reversal \\
\hline Blue & Direct S-wave & curved & continuous & 0.298 & 0.074 & -3800 & Polarity reversal \\
\hline Green & Reflection & slightly curved & continuous & 0.330 & 0.385 & 15490 & \\
\hline Yellow & Reflection & curved & continuous & 0.360 & 0.430 & 12170 & \\
\hline Purple & Reflection & curved & mixed, $\geq 2$ & 0.440 & 0.491 & 16710 & \\
\hline Orange & Reflection & curved & mixed, $\geq 2$ & 0.453 & 0.568 & 7410 & \\
\hline Light Blue & Reflection & curved & mixed, 2 & 0.530 & 0.633 & 8270 & \\
\hline White & Reflection & curved & continuous & 0.593 & 0.700 & 7960 & \\
\hline
\end{tabular}




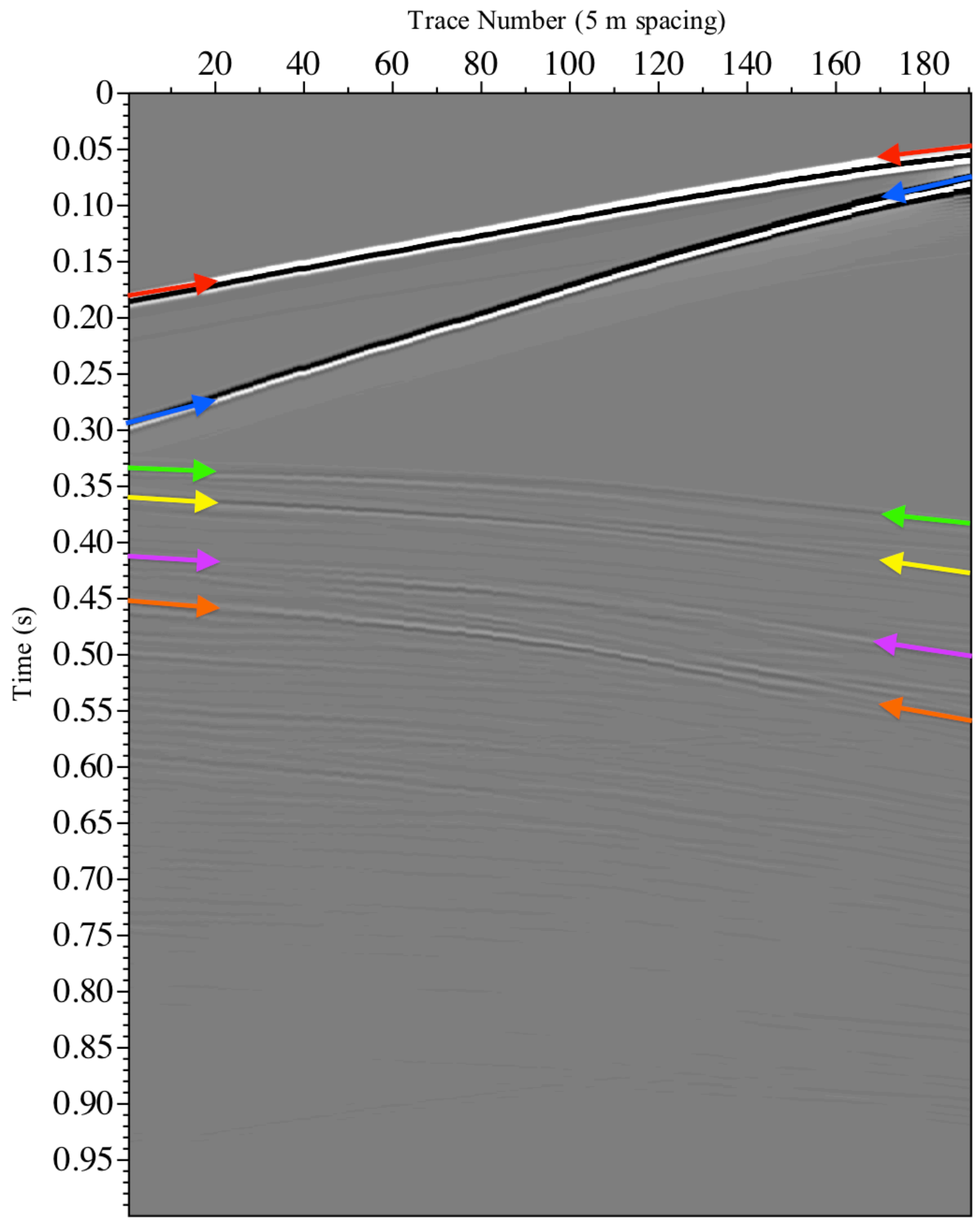

Figure 4.2.5 - Zero-offset northing particle velocity synthetic VSP shot-gather from 4Q66W3 in the 'Binary' model with prominent reflections identified by coloured arrows. 
Table 4.2.4 - Summary of annotated events in the northing particle velocity shot gather from the 'Binary' model shown in Figure 4.2.5.

\begin{tabular}{|c|c|c|c|c|c|c|c|}
\hline \begin{tabular}{|l} 
Model \\
Data set \\
Figure \\
\end{tabular} & $\begin{array}{l}\text { 'Binary' } \\
\text { Northing particle v } \\
4.2 .5\end{array}$ & elocity & & & & & \\
\hline Arrow Colour & Direct / Reflected & Alignment & Continuity & $\begin{array}{c}\text { Trace } 1 \\
\text { (s) }\end{array}$ & $\begin{array}{c}\text { Trace } 190 \\
\text { (s) }\end{array}$ & \begin{tabular}{|c|}
$\begin{array}{c}\text { Apparent velocity } \\
(\mathrm{m} / \mathrm{s})\end{array}$ \\
\end{tabular} & Notes \\
\hline Red & Direct P-wave & slightly curved & continuous & 0.180 & 0.042 & -6180 & \\
\hline Blue & Direct S-wave & curved & continuous & 0.298 & 0.074 & -3800 & \\
\hline Green & Reflection & slightly curved & mixed, 2 & 0.331 & 0.385 & 15780 & \\
\hline Yellow & Reflection & curved & continuous & 0.360 & 0.430 & 12170 & \\
\hline Purple & Reflection & curved & mixed, $\geq 2$ & 0.410 & 0.506 & 8880 & \\
\hline Orange & Reflection & curved & continuous & 0.451 & 0.560 & 7820 & \\
\hline
\end{tabular}

The vertical particle velocity shot gather from the 'Binary' model (Table 4.2.5 and Figure 4.2.6) shows a slightly stronger direct $\mathrm{P}$-wave than the direct $\mathrm{S}$-wave, as would be expected from the geometry of the shot and receiver array. The direct S-wave appears to lose amplitude as it travels deeper and no polarity reversals in the direct waves are observed. Qualitatively, reflections are the most numerous in this shot gather and many of the same reflections seen in other shot gathers are present with varying apparent velocities. The same imaging artifact is observed as are some mixed or overlapping reflections. There appears to be three distinct intervals of strong reflectivity in Figure 4.2.6, roughly between 0.330 and $0.430 \mathrm{~s}$ (green and yellow arrows), 0.410 and $0.560 \mathrm{~s}$ (purple and orange arrows) and 0.590 and $0.670 \mathrm{~s}$ (light blue arrows). 


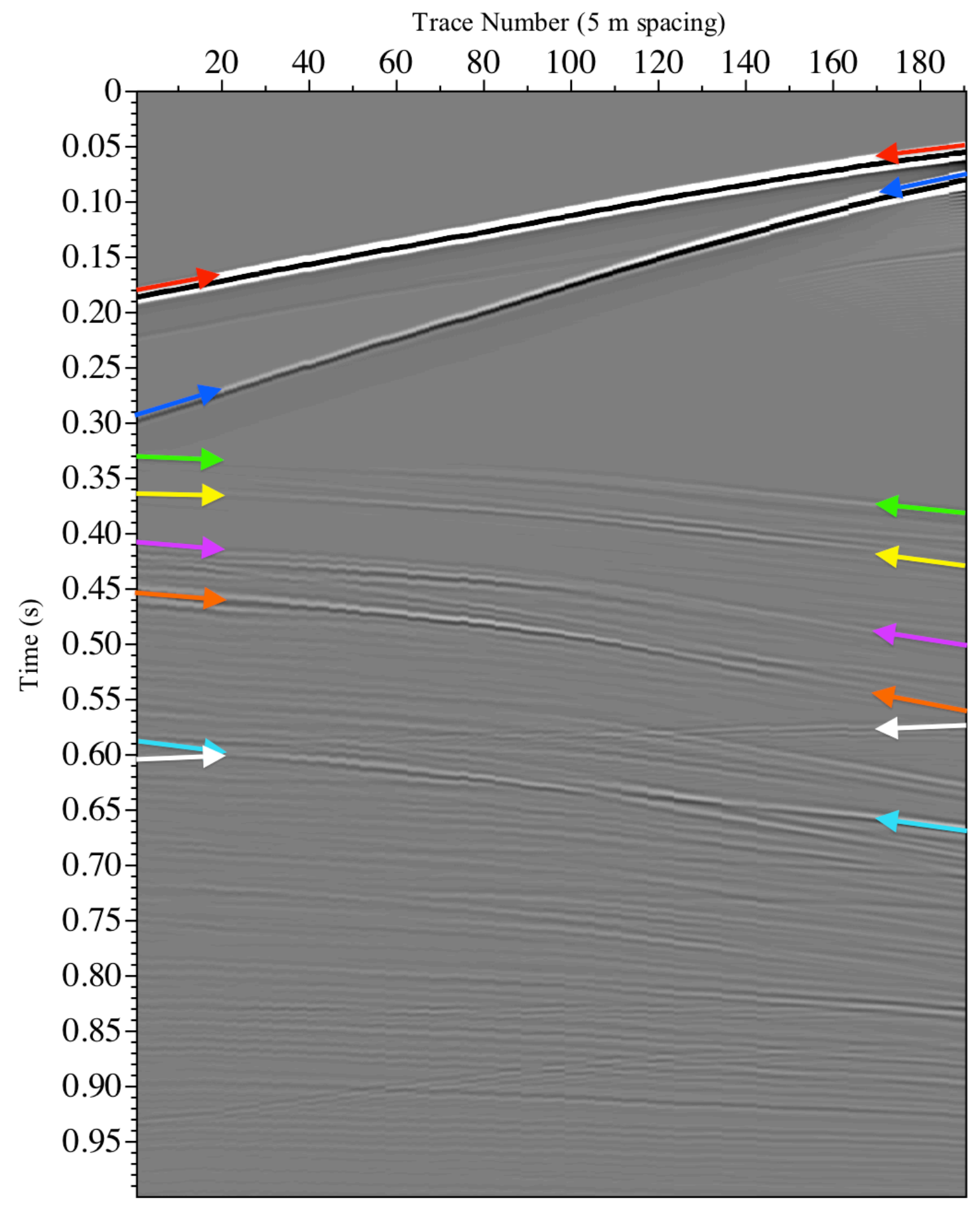

Figure 4.2.6 - Zero-offset vertical particle velocity synthetic VSP shot-gather from 4Q66W3 in the 'Binary' model with prominent reflections identified by coloured arrows. 
Table 4.2.5 - Summary of annotated events in the vertical particle velocity shot gather from the 'Binary' model shown in Figure 4.2.6.

\begin{tabular}{|c|c|c|c|c|c|c|c|}
\hline $\begin{array}{l}\text { Model } \\
\text { Data set } \\
\text { Figure }\end{array}$ & $\begin{array}{l}\text { 'Binary' } \\
\text { Vertical particle vel } \\
4.2 .6\end{array}$ & locity & & & & & \\
\hline Arrow Colour & Direct / Reflected & Alignment & Continuity & $\begin{array}{c}\text { Trace } 1 \\
\text { (s) }\end{array}$ & \begin{tabular}{|c|} 
Trace 190 \\
(s) \\
\end{tabular} & $\begin{array}{c}\text { Apparent velocity } \\
(\mathrm{m} / \mathrm{s})\end{array}$ & Notes \\
\hline Red & Direct P-wave & slightly curved & continuous & 0.180 & 0.042 & -6180 & \\
\hline Blue & Direct S-wave & curved & continuous & 0.298 & 0.074 & -3800 & weaker with depth \\
\hline Green & Reflection & curved & continuous & 0.328 & 0.384 & 15220 & \\
\hline Yellow & Reflection & curved & continuous & 0.364 & 0.430 & 12910 & \\
\hline Purple & Reflection & curved & continuous & 0.408 & 0.501 & 9160 & \\
\hline Orange & Reflection & curved & continuous & 0.452 & 0.563 & 7680 & \\
\hline Light blue & Reflection & curved & mixed, 2 & 0.585 & 0.672 & 9790 & \\
\hline White & Artifact & linear & continuous & 0.604 & 0.569 & -24350 & \\
\hline
\end{tabular}

Four modified versions of the 'Binary' model were then used in similar simulations, each including a single stratigraphic level of ore lenses. The results of these models should constrain the origin of some reflections within the 'Binary' model. For comparison, vertical particle velocity shot gathers for each of the models and tables summarizing their annotated reflections are displayed and discussed here. The direct P- and S- waves for all of these shot gathers are identical and the previously-mentioned image artifact is seen in each. The 'Binary (Callinan North)' shot gather shows three distinct intervals of relatively strong reflections as was seen in the 'Binary' model results. The shot gather from the 'Binary (Callinan East)' model also shows three similar intervals of strong reflections, but with slightly different timing and apparent velocities. In both shot gathers, the observed reflections are all continuous and most have relatively slow apparent velocities. Many weaker reflections are also seen throughout both of the shot gathers. 


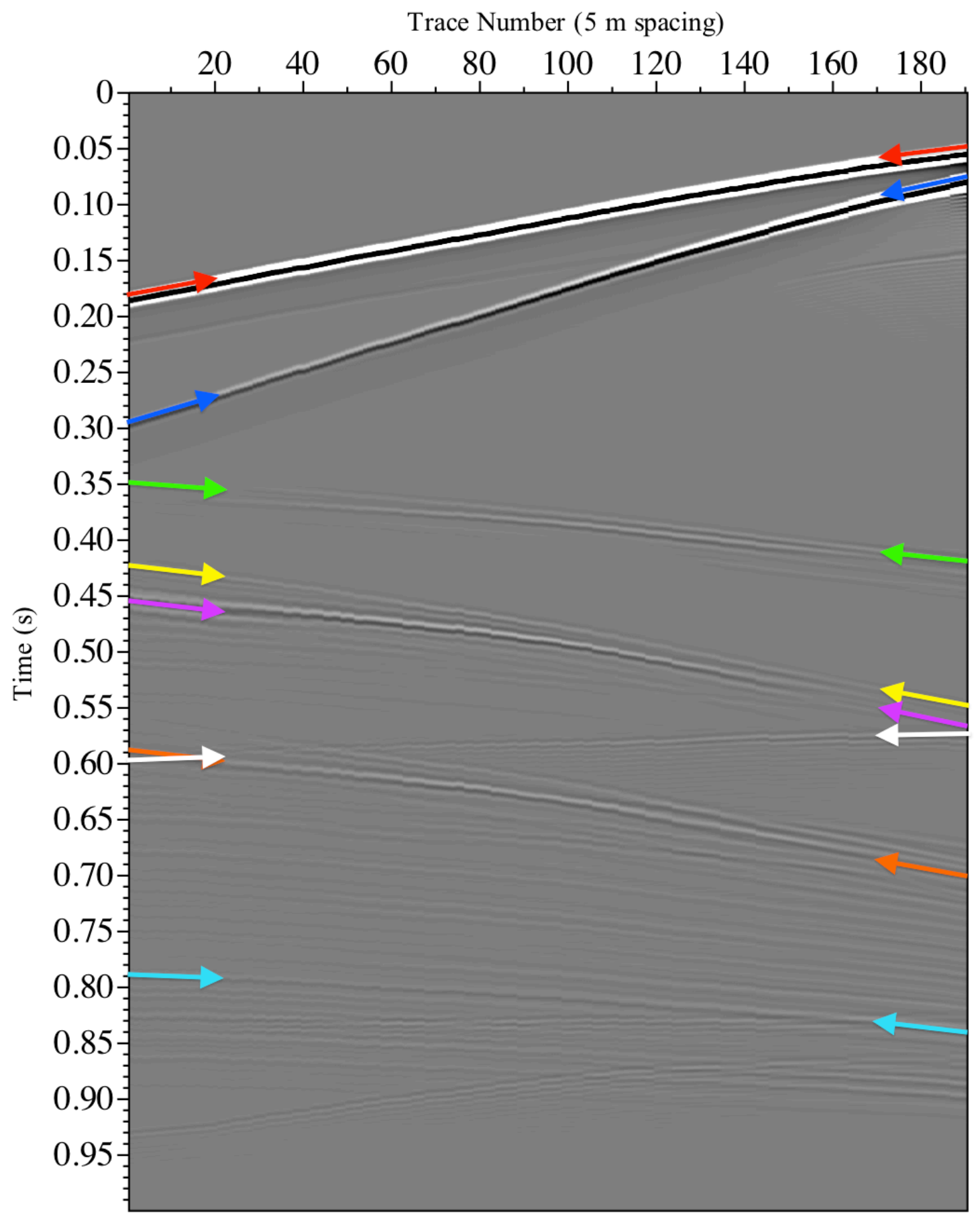

Figure 4.2.7 - Zero-offset vertical particle velocity synthetic VSP shot-gather from 4Q66W3 in the 'Binary (Callinan North)' model with prominent reflections identified by coloured arrows. 
Table 4.2.6 - Summary of annotated events in the vertical particle velocity shot gather from the 'Binary (Callinan North)' model shown in Figure 4.2.7.

\begin{tabular}{|l|l|l|l|c|c|c|c|}
\hline $\begin{array}{l}\text { Model } \\
\text { Data set } \\
\text { Figure }\end{array}$ & $\begin{array}{l}\text { 'Binary' Callinan North } \\
\text { Vertical particle velocity } \\
4.2 .7\end{array}$ \\
\hline Arrow Colour & Direct / Reflected & Alignment & Continuity & $\begin{array}{c}\text { Trace 1 } \\
(\mathbf{s})\end{array}$ & $\begin{array}{c}\text { Trace 190 } \\
(\mathbf{s})\end{array}$ & $\begin{array}{c}\text { Apparent velocity } \\
(\mathbf{m} / \mathbf{s})\end{array}$ & Notes \\
\hline Red & Direct P-wave & slightly curved & continuous & 0.180 & 0.042 & -6180 & \\
\hline Blue & Direct S-wave & curved & continuous & 0.298 & 0.074 & -3800 & weaker with depth \\
\hline Green & Reflection & slightly curved & continuous & 0.348 & 0.420 & 11840 & \\
\hline Yellow & Reflection & curved & continuous & 0.422 & 0.550 & 6660 & \\
\hline Purple & Reflection & curved & continuous & 0.454 & 0.569 & 7410 & \\
\hline Orange & Reflection & curved & continuous & 0.588 & 0.700 & 7610 & \\
\hline White & Artifact & linear & continuous & 0.600 & 0.569 & -27490 & \\
\hline Light blue & Reflection & curved & continuous & 0.588 & 0.700 & 7610 & \\
\hline
\end{tabular}




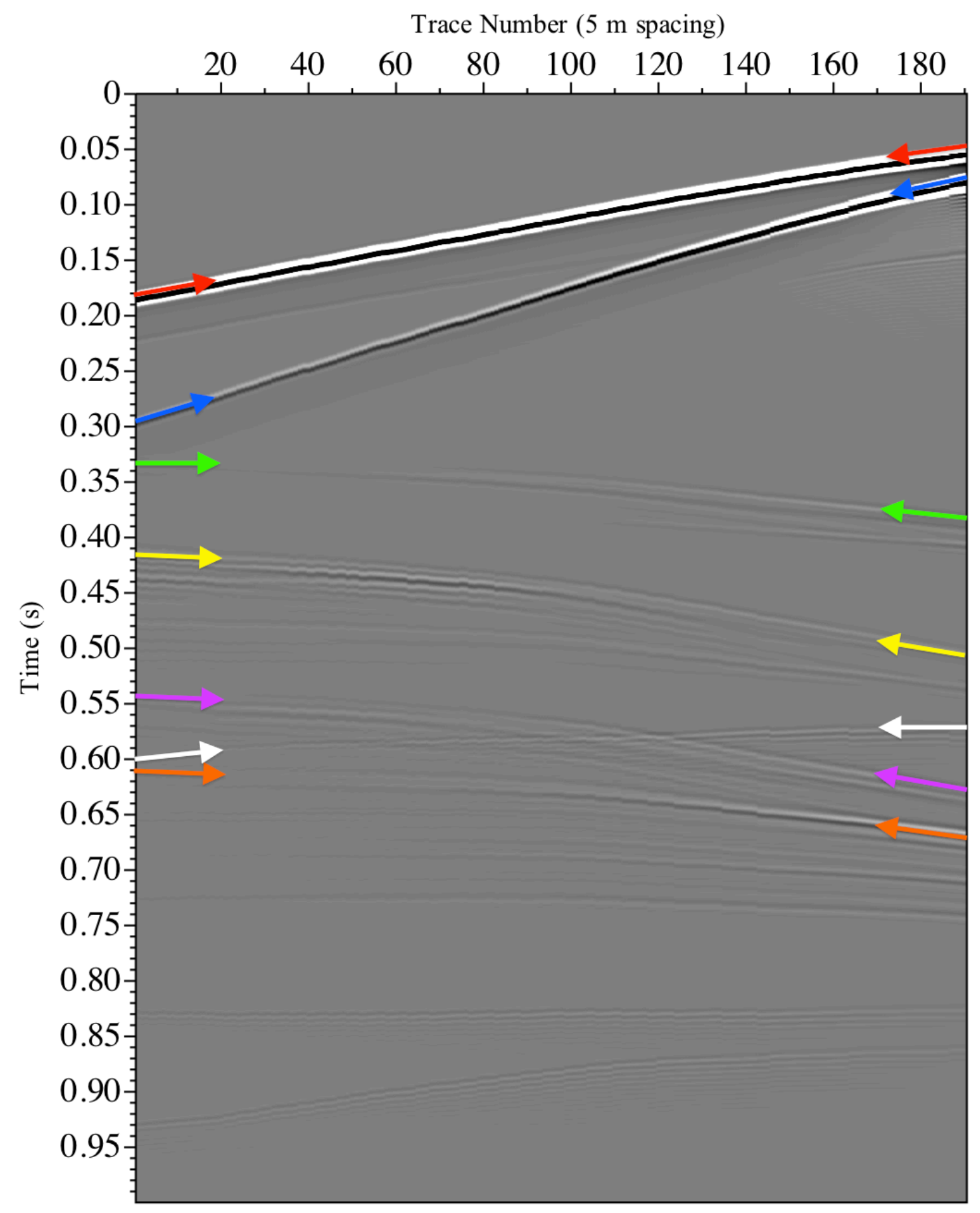

Figure 4.2.8 - Zero-offset vertical particle velocity synthetic VSP shot-gather from 4Q66W3 in the 'Binary (Callinan East)' model with prominent reflections identified by coloured arrows. 
Table 4.2.7 - Summary of annotated events in the vertical particle velocity shot gather from the 'Binary (Callinan East)' model shown in Figure 4.2.8.

\begin{tabular}{|c|c|c|c|c|c|c|c|}
\hline $\begin{array}{l}\text { Model } \\
\text { Data set } \\
\text { Figure }\end{array}$ & $\begin{array}{l}\text { 'Binary' Callinan Ea } \\
\text { Vertical particle vel } \\
4.2 .8\end{array}$ & $\begin{array}{l}\text { ast } \\
\text { locity }\end{array}$ & & & & & \\
\hline Arrow Colour & Direct / Reflected & Alignment & Continuity & $\begin{array}{c}\text { Trace } 1 \\
\text { (s) }\end{array}$ & $\begin{array}{c}\text { Trace } 190 \\
\text { (s) }\end{array}$ & $\begin{array}{c}\text { Apparent velocity } \\
(\mathrm{m} / \mathrm{s})\end{array}$ & Notes \\
\hline Red & Direct P-wave & slightly curved & continuous & 0.180 & 0.042 & -6180 & \\
\hline Blue & Direct S-wave & curved & continuous & 0.298 & 0.074 & -3800 & weaker with depth \\
\hline Green & Reflection & curved & continuous & 0.331 & 0.385 & 15780 & \\
\hline Yellow & Reflection & curved & continuous & 0.412 & 0.509 & 8790 & stronger deep \\
\hline \begin{tabular}{|l|} 
Purple \\
\end{tabular} & Reflection & curved & continuous & 0.542 & 0.628 & 9910 & artifact interuption \\
\hline White & Artifact & linear & continuous & 0.601 & 0.568 & -25820 & \\
\hline Orange & Reflection & curved & continuous & 0.608 & 0.672 & 13310 & stronger shallow \\
\hline
\end{tabular}

In general, reflections are much weaker in the 'Binary (Callinan South)' vertical particle velocity shot gather (Table 4.2.8 and Figure 4.2.9) than in the 'Binary (Callinan North)' or 'Binary (Callinan East) shot gathers. Again, the reflections are all continuous and have a wide range of apparent velocities. Previously-observed reflections occurring between approximately 0.330 and $0.430 \mathrm{~s}$ are not seen in this shot gather. Also, weak reflections are seen as late as $0.900 \mathrm{~s}$, later than either of the previous shot gathers. Reflectivity in the 'Binary (777)' shot gather (Table 4.2.9 and Figure 4.2.10) is even weaker than in the 'Binary (Callinan South)' shot gather, and no distinct reflections are annotated in Figure 4.2.10. There are weak reflections present throughout both of these shot gathers. 


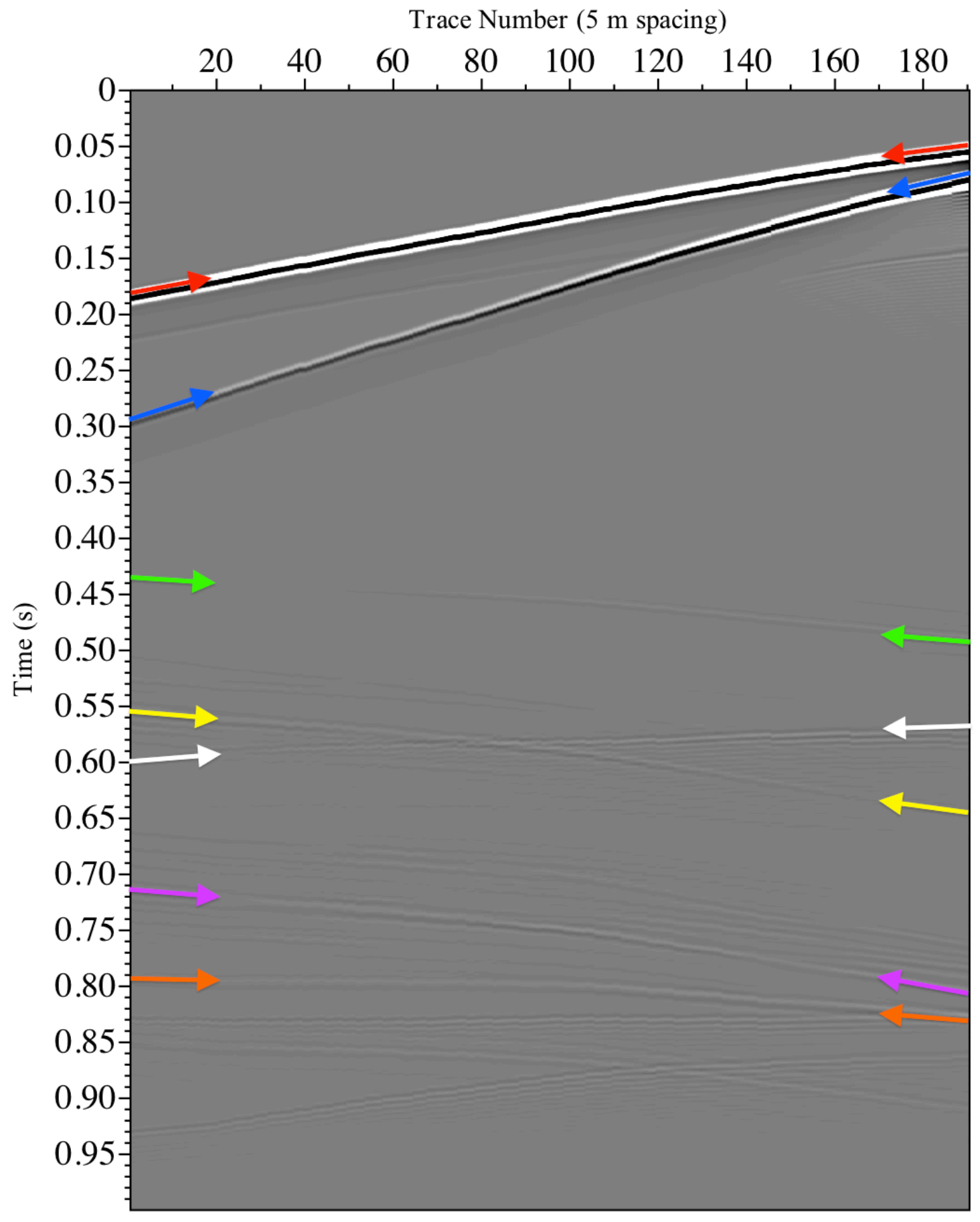

Figure 4.2.9 - Zero-offset vertical particle velocity synthetic VSP shot-gather from 4Q66W3 in the 'Binary (Callinan South)' model with prominent reflections identified by coloured arrows. 
Table 4.2.8 - Summary of annotated events in the vertical particle velocity shot gather from the 'Binary (Callinan South)' model shown in Figure 4.2.9.

\begin{tabular}{|c|c|c|c|c|c|c|c|}
\hline $\begin{array}{l}\text { Model } \\
\text { Data set } \\
\text { Figure } \\
\end{array}$ & $\begin{array}{l}\text { 'Binary' Callinan Sc } \\
\text { Vertical particle vel } \\
4.2 .9\end{array}$ & $\begin{array}{l}\text { outh } \\
\text { locity }\end{array}$ & & & & & \\
\hline Arrow Colour & Direct / Reflected & Alignment & Continuity & $\begin{array}{c}\text { Trace } 1 \\
(\mathrm{~s})\end{array}$ & \begin{tabular}{|c|} 
Trace 190 \\
(s) \\
\end{tabular} & \begin{tabular}{|c|}
$\begin{array}{c}\text { Apparent velocity } \\
(\mathrm{m} / \mathrm{s})\end{array}$ \\
\end{tabular} & Notes \\
\hline Red & Direct P-wave & slightly curved & continuous & 0.180 & 0.042 & -6180 & \\
\hline Blue & Direct S-wave & curved & continuous & 0.298 & 0.074 & -3800 & weaker with depth \\
\hline Green & Reflection & slightly curved & continuous & 0.435 & 0.495 & 14200 & very weak \\
\hline Yellow & Reflection & curved & continuous & 0.555 & 0.647 & 9260 & weak \\
\hline White & Artifact & linear & continuous & 0.600 & 0.568 & -26630 & \\
\hline Purple & Reflection & curved & continuous & 0.713 & 0.808 & 8970 & weak \\
\hline Orange & Reflection & slightly curved & continuous & 0.792 & 0.834 & 20290 & weak \\
\hline
\end{tabular}




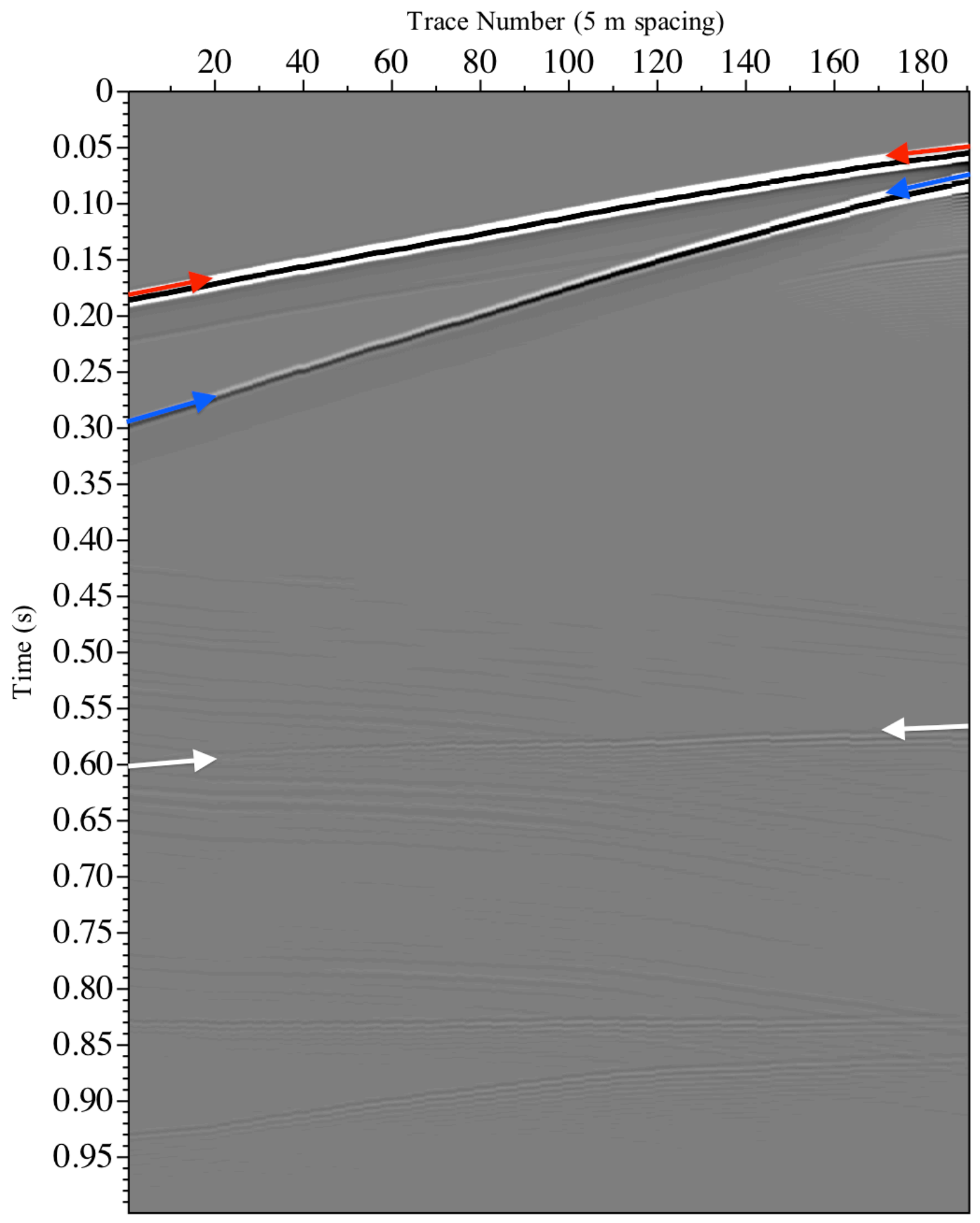

Figure 4.2.10 - Zero-offset vertical particle velocity synthetic VSP shot-gather from 4Q66W3 in the 'Binary (777)' model. 
Table 4.2.9 - Summary of annotated events in the vertical particle velocity shot gather from the 'Binary (777)’ model shown in Figure 4.2.9.

\begin{tabular}{|l|l|l|l|c|c|c|c|}
\hline $\begin{array}{l}\text { Model } \\
\text { Data set } \\
\text { Figure }\end{array}$ & $\begin{array}{l}\text { Vinary' } 777 \\
\text { Vertical particle velocity }\end{array}$ \\
\hline Arrow Colour & Direct / Reflected & Alignment & Continuity & $\begin{array}{c}\text { Trace 1 } \\
(\mathbf{s})\end{array}$ & $\begin{array}{c}\text { Trace 190 } \\
(\mathbf{s})\end{array}$ & $\begin{array}{c}\text { Apparent velocity } \\
(\mathbf{m} / \mathbf{s})\end{array}$ & Notes \\
\hline Red & Direct P-wave & slightly curved & continuous & 0.180 & 0.042 & -6180 & \\
\hline Blue & Direct S-wave & curved & continuous & 0.298 & 0.074 & -3800 & weaker with depth \\
\hline White & Artifact & linear & continuous & 0.601 & 0.562 & -21850 & \\
\hline
\end{tabular}

Divergence and rotation 3D wavefield visualizations of the 'Binary' model demonstrate the high contrast in acoustic impedance between the ore lenses and the basalt background. The contrasting strengths of the direct P- and S-waves in each case also show the effectiveness of these visualizations in differentiating these waves. It is also obvious that several distinct reflection wavefronts are produced, each originating from different groups of ore lenses. In the divergence 3D wavefield (Figure 4.2.11), all reflections seen at $0.260 \mathrm{~s}$ must be P-P since the direct shear wave has not yet arrived. For the same reason, all reflections seen in the rotation 3D wavefield (Figure 4.2.12) at $0.260 \mathrm{~s}$ must be P-S conversions. 


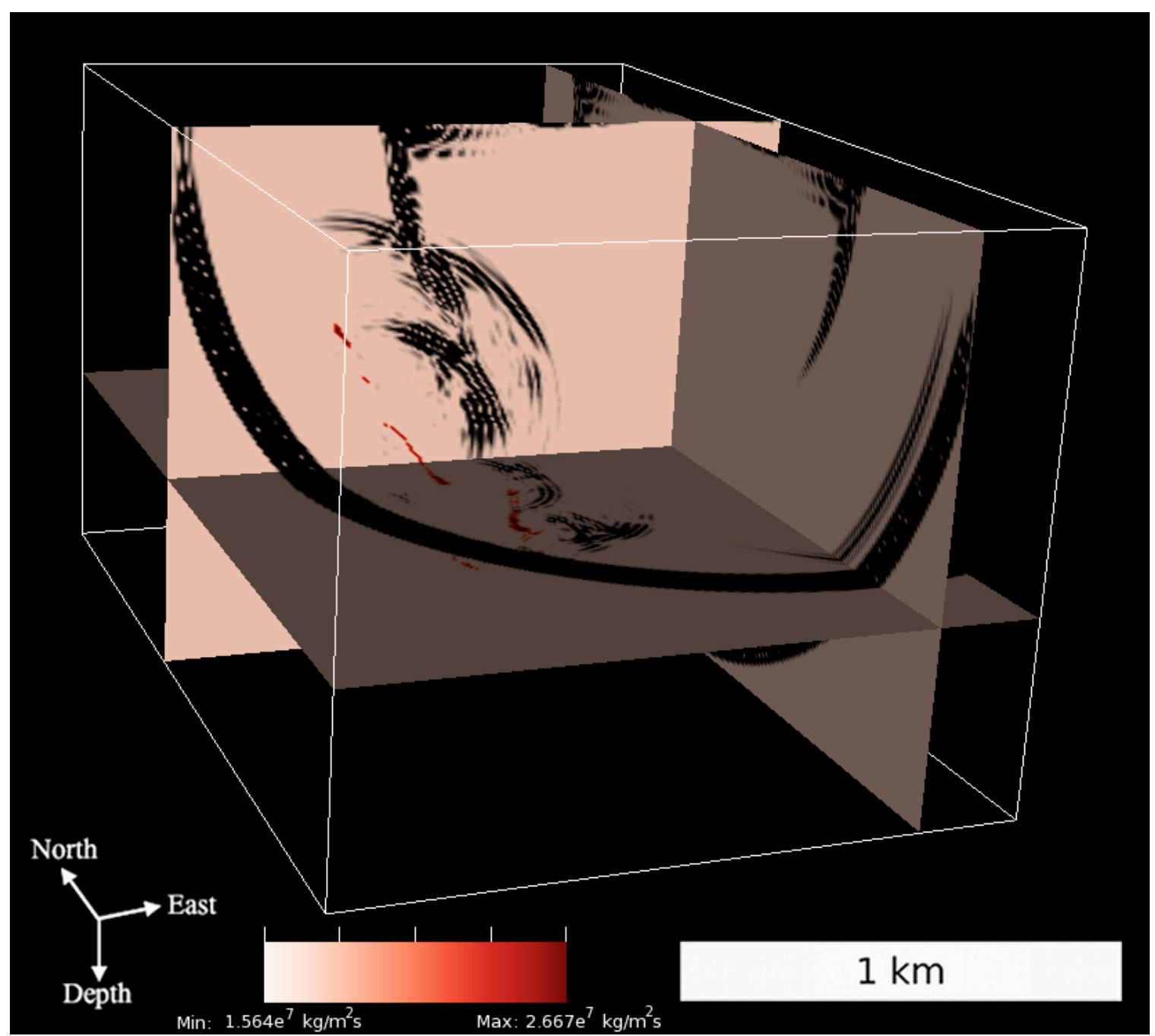

Figure 4.2.11 - 3D divergence wavefield visualization overlain on acoustic impedance

from the 'Binary' model at $0.260 \mathrm{~s}$. The location of the source is approximated by the intersection of the vertical planes and the surface. The location of the receiver array is approximated by the vertical intersection of the two vertical planes above the horizontal plane. Darker colours indicate voxels with higher acoustic impedance. 


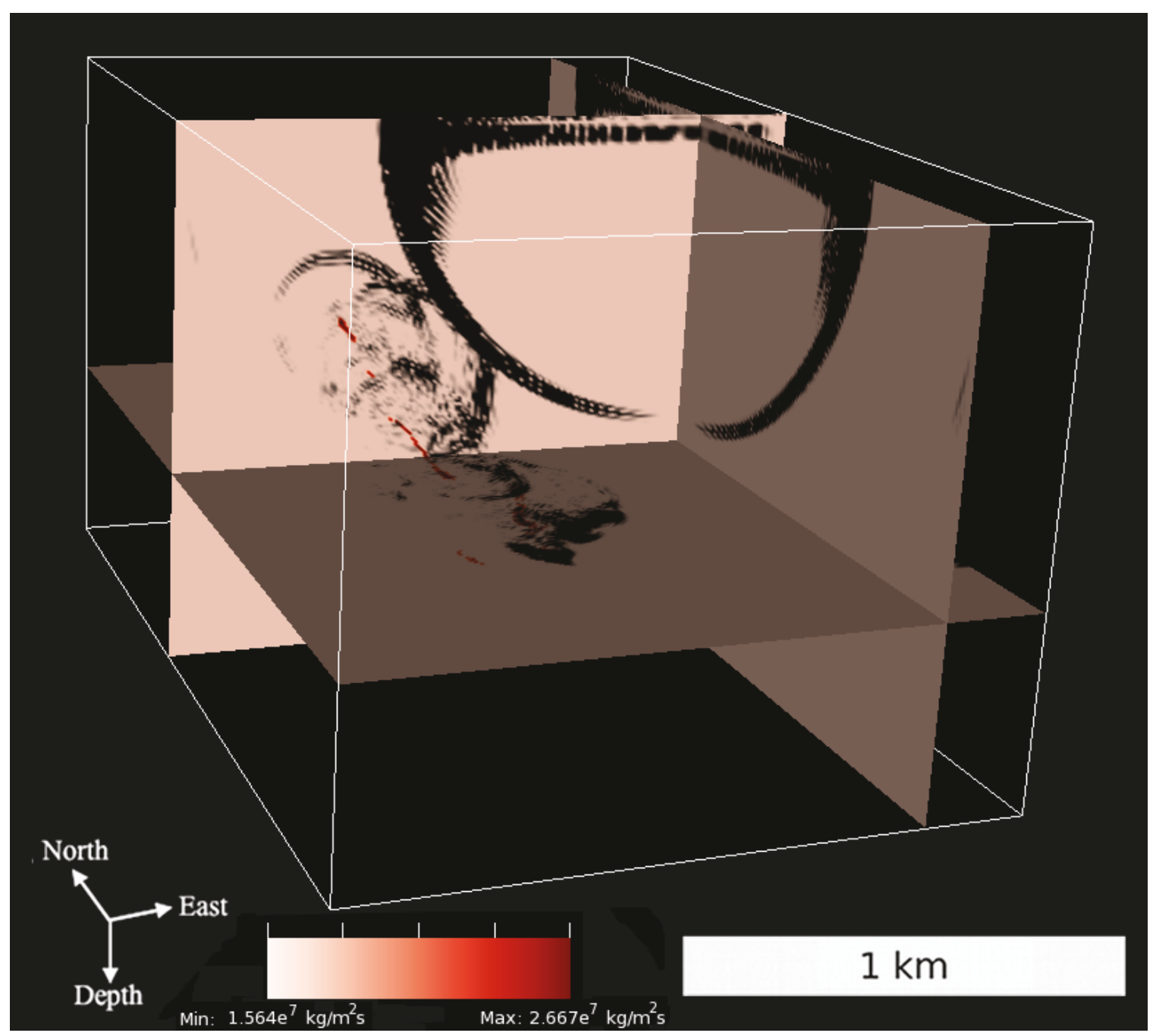

Figure 4.2.12 - 3D rotation wavefield visualization overlain on acoustic impedance from

the 'Binary' model at $0.260 \mathrm{~s}$. The location of the source is approximated by the

intersection of the vertical planes and the surface. The location of the receiver array is approximated by the vertical intersection of the two vertical planes above the horizontal plane. Darker colours indicate voxels with higher acoustic impedance. 


\section{3 - 'Ore/Missi' model observations}

For comparison, only vertical particle velocity shot gathers from each of the other models are shown and discussed here. The shot gather from the 'Ore/Missi' model is more complex than that of the 'Binary' model. Similar reflections to those from the 'Binary' model are observed but added to this are events related to the presence of the Missi metasedimentary basin. The most obvious new feature is a series of strong reverberating reflections traveling downwards through the receiver array shortly after the direct P- and S-waves. Two distinct groups are seen; one with a faster apparent velocity and one with a slower apparent velocity (green and yellow arrows in Figure 4.3.1, respectively). The three distinct intervals of reflections seen in the 'Binary' model shot gathers (green, yellow, purple, orange and light blue arrows in Figure 4.2.6) are present, but there appears to be some variation in the recorded waves as well as at least one new reflection (white arrows in Figure 4.3.1). Also, very late in the shot gather another new reflection (dark green arrows in Figure 4.3.1) is observed traveling downwards through the receiver array. 


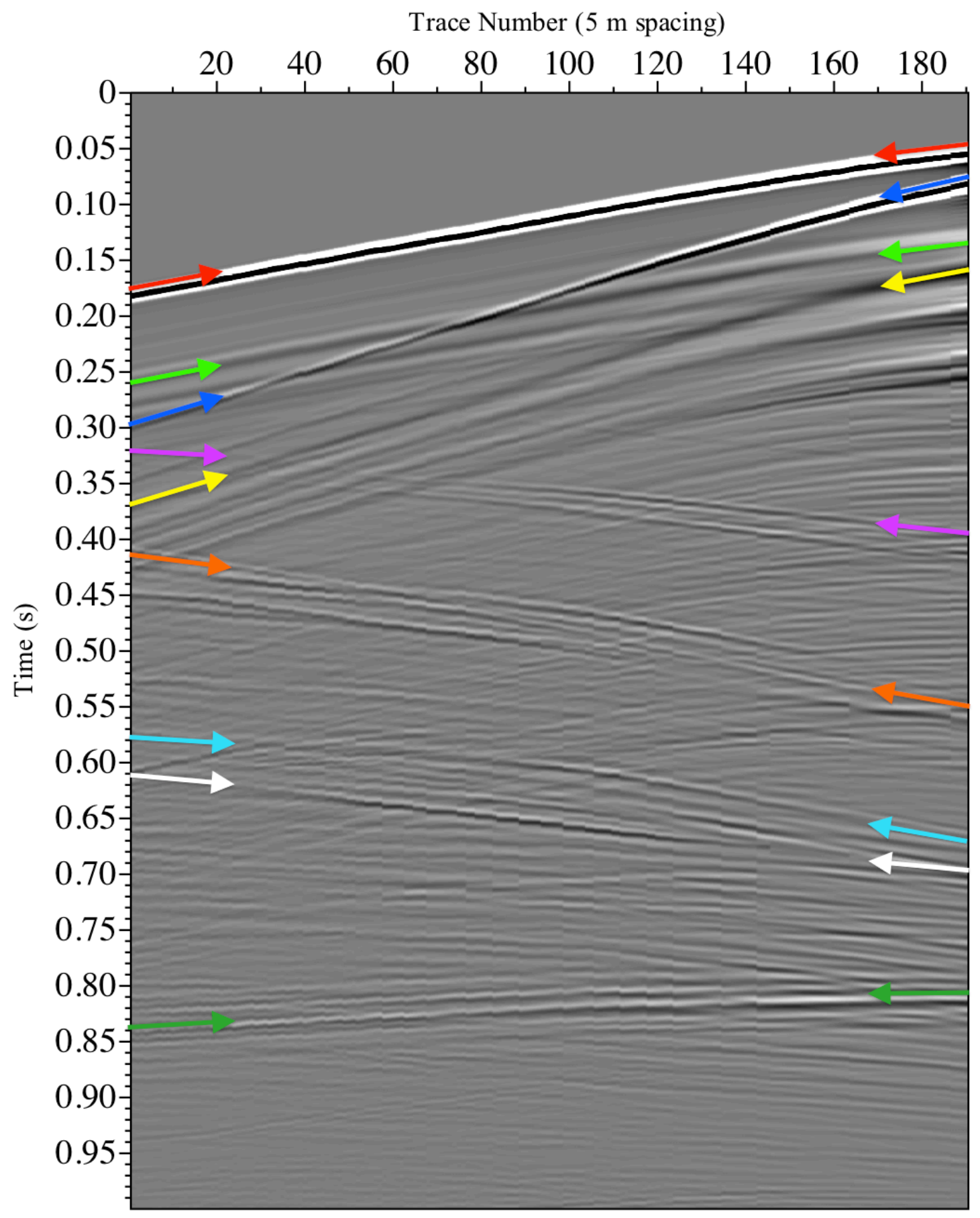

Figure 4.3.1 - Zero-offset vertical particle velocity synthetic VSP shot-gather from 4Q66W3 in the 'Ore/Missi' model with prominent reflections identified by coloured arrows. 
Table 4.3.1 - Summary of annotated events in the vertical particle velocity shot gather from the 'Ore/Missi' model shown in Figure 4.3.1.

\begin{tabular}{|c|c|c|c|c|c|c|c|}
\hline $\begin{array}{l}\text { Model } \\
\text { Data set } \\
\text { Figure }\end{array}$ & $\begin{array}{l}\text { 'Ore / Missi' } \\
\text { Vertical particle ve } \\
4.3 .1\end{array}$ & locity & & & & & \\
\hline Arrow Colour & Direct / Reflected & Alignment & Continuity & $\begin{array}{c}\text { Trace } 1 \\
\text { (s) }\end{array}$ & \begin{tabular}{|c|} 
Trace 190 \\
(s) \\
\end{tabular} & $\begin{array}{c}\text { Apparent velocity } \\
(\mathrm{m} / \mathrm{s})\end{array}$ & Notes \\
\hline Red & Direct P-wave & slightly curved & continuous & 0.180 & 0.042 & -6180 & \\
\hline Blue & Direct S-wave & curved & continuous & 0.298 & 0.074 & -3800 & weaker with depth \\
\hline Green & Reflection & slightly curved & continuous & 0.260 & 0.137 & -6930 & several peaks \\
\hline Purple & Reflection & slightly curved & continuous & 0.320 & 0.397 & 11070 & \\
\hline Yellow & Reflection & curved & continuous & 0.370 & 0.158 & -4020 & several peaks \\
\hline Orange & Reflection & curved & mixed,$\geq 2$ & 0.412 & 0.550 & 6180 & \\
\hline Light blue & Reflection & curved & continuous & 0.577 & 0.672 & 8970 & \\
\hline White & Reflection & linear & continuous & 0.611 & 0.698 & 9790 & \\
\hline Dark green & Reflection & slightly curved & continuous & 0.839 & 0.803 & -23670 & several peaks \\
\hline
\end{tabular}

\section{4 - 'Lithological' model observations}

The vertical particle velocity shot gather from the 'Lithological' model appears very similar to that from the 'Ore/Missi' model. The same reverberating reflections following the direct P- and S-waves can be seen (green and yellow arrows in Figure 4.4.1). The three reflection intervals and the late downward-traveling wave are also seen again in this shot gather. In fact, there appears to be only very slight differences in these shot gathers, which is surprising considering the volume of voxels with different physical properties in these two models. 


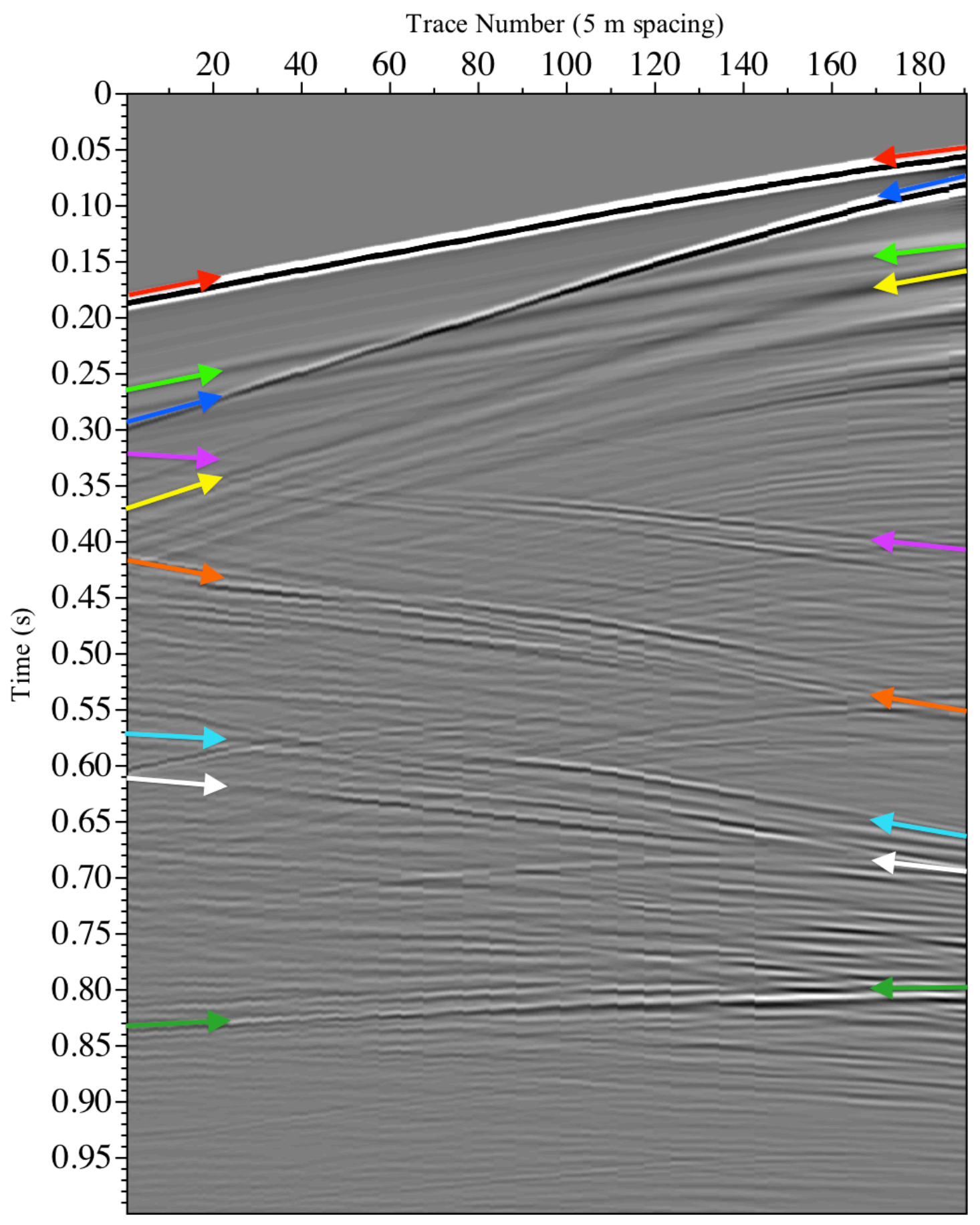

Figure 4.4.1 - Zero-offset vertical particle velocity synthetic VSP shot-gather from 4Q66W3 in the 'Lithological' model with prominent reflections identified by coloured arrows. 
Table 4.4.1 - Summary of annotated events in the vertical particle velocity shot gather from the 'Lithological' model shown in Figure 4.4.1.

\begin{tabular}{|c|c|c|c|c|c|c|c|}
\hline $\begin{array}{l}\text { Model } \\
\text { Data set } \\
\text { Figure }\end{array}$ & $\begin{array}{l}\text { 'Lithological' } \\
\text { Vertical particle vel } \\
4.4 .1\end{array}$ & locity & & & & & \\
\hline Arrow Colour & Direct / Reflected & Alignment & Continuity & $\begin{array}{c}\text { Trace } 1 \\
\text { (s) }\end{array}$ & \begin{tabular}{|c|} 
Trace 190 \\
(s) \\
\end{tabular} & $\begin{array}{c}\text { Apparent velocity } \\
(\mathrm{m} / \mathrm{s})\end{array}$ & Notes \\
\hline Red & Direct P-wave & slightly curved & continuous & 0.180 & 0.042 & -6180 & \\
\hline Blue & Direct S-wave & curved & continuous & 0.298 & 0.074 & -3800 & weaker with depth \\
\hline Green & Reflection & slightly curved & continuous & 0.262 & 0.132 & -6560 & several peaks \\
\hline Purple & Reflection & slightly curved & continuous & 0.321 & 0.410 & 9570 & \\
\hline Yellow & Reflection & curved & continuous & 0.370 & 0.155 & -3960 & several peaks \\
\hline Orange & Reflection & curved & mixed, $\geq 2$ & 0.418 & 0.556 & 6180 & \\
\hline Light blue & Reflection & curved & continuous & 0.570 & 0.661 & 9360 & \\
\hline White & Reflection & linear & continuous & 0.610 & 0.696 & 9910 & \\
\hline Dark green & Reflection & slightly curved & continuous & 0.835 & 0.799 & -23670 & several peaks \\
\hline
\end{tabular}

The 3D wavefield visualizations for the 'Lithological' model are very helpful to determine the origin of reflections within the modeled volume. At $0.260 \mathrm{~s}$ we can see P$\mathrm{P}$ and P-S reflections traveling back toward the receiver array in the divergence (Figure 4.4.2) and rotation (Figure 4.4.3) wavefield visualizations, respectively. These reflections appear considerably more complex than those seen previously in the 'Binary' model at the same time step, but they still are centered about the location of ore lenses. We can also see new downgoing reflections approaching the intersection of the three planes from above in Figure 4.4.2 and 4.4.3. It is also worth noting that the major contrasts in acoustic impedance in the 'Lithological' model are the Missi metasedimentary basin and the ore lenses. Contrasts in acoustic impedance of the ore-hosting horizon are less noticeable. 


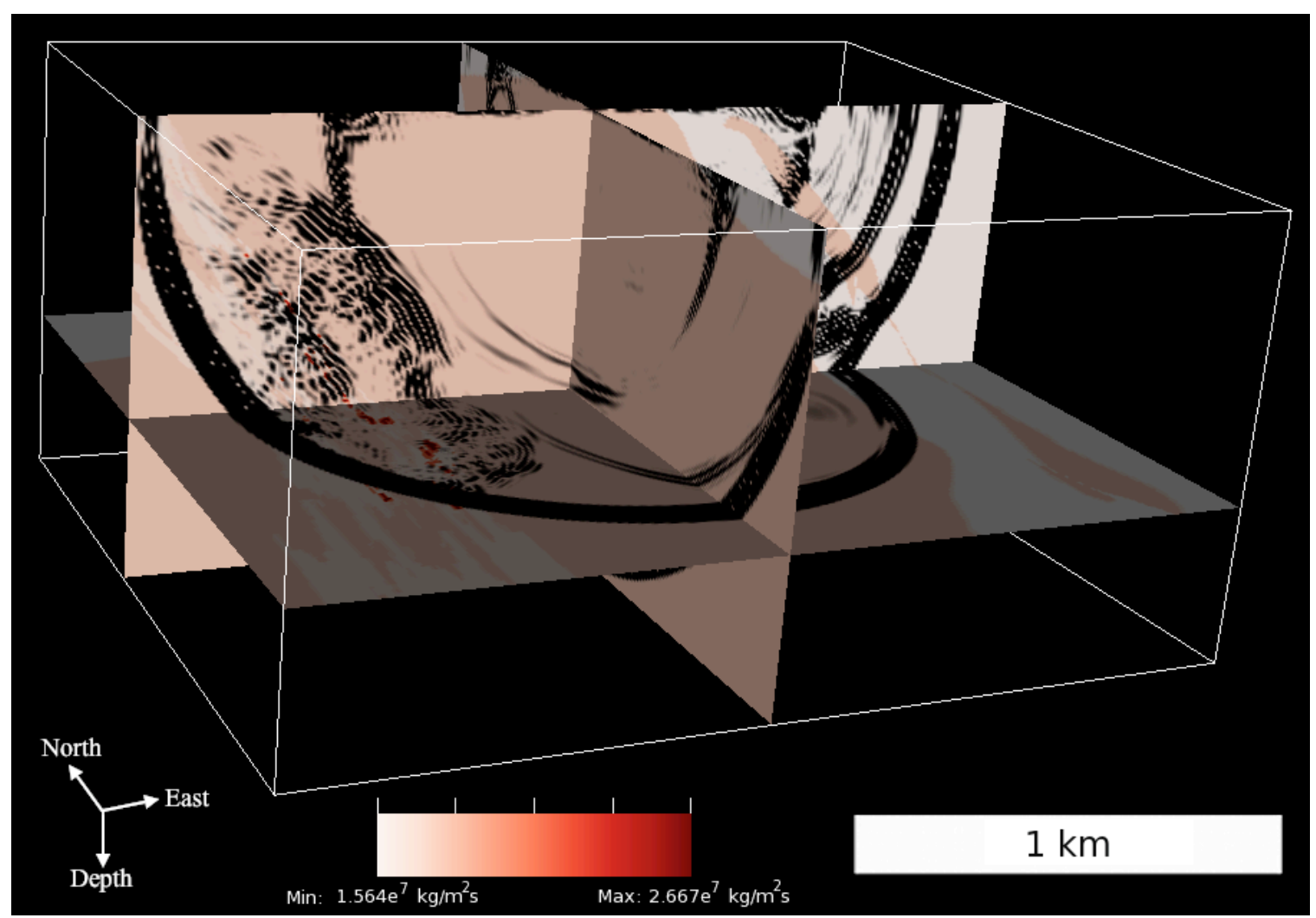

Figure 4.4.2 - 3D divergence wavefield visualization overlain on acoustic impedance

from the 'Lithological' model at $0.260 \mathrm{~s}$. The location of the source is approximated by the intersection of the vertical planes and the surface. The location of the receiver array is approximated by the vertical intersection of the two vertical planes above the horizontal plane. Darker colours indicate voxels with higher acoustic impedance. 


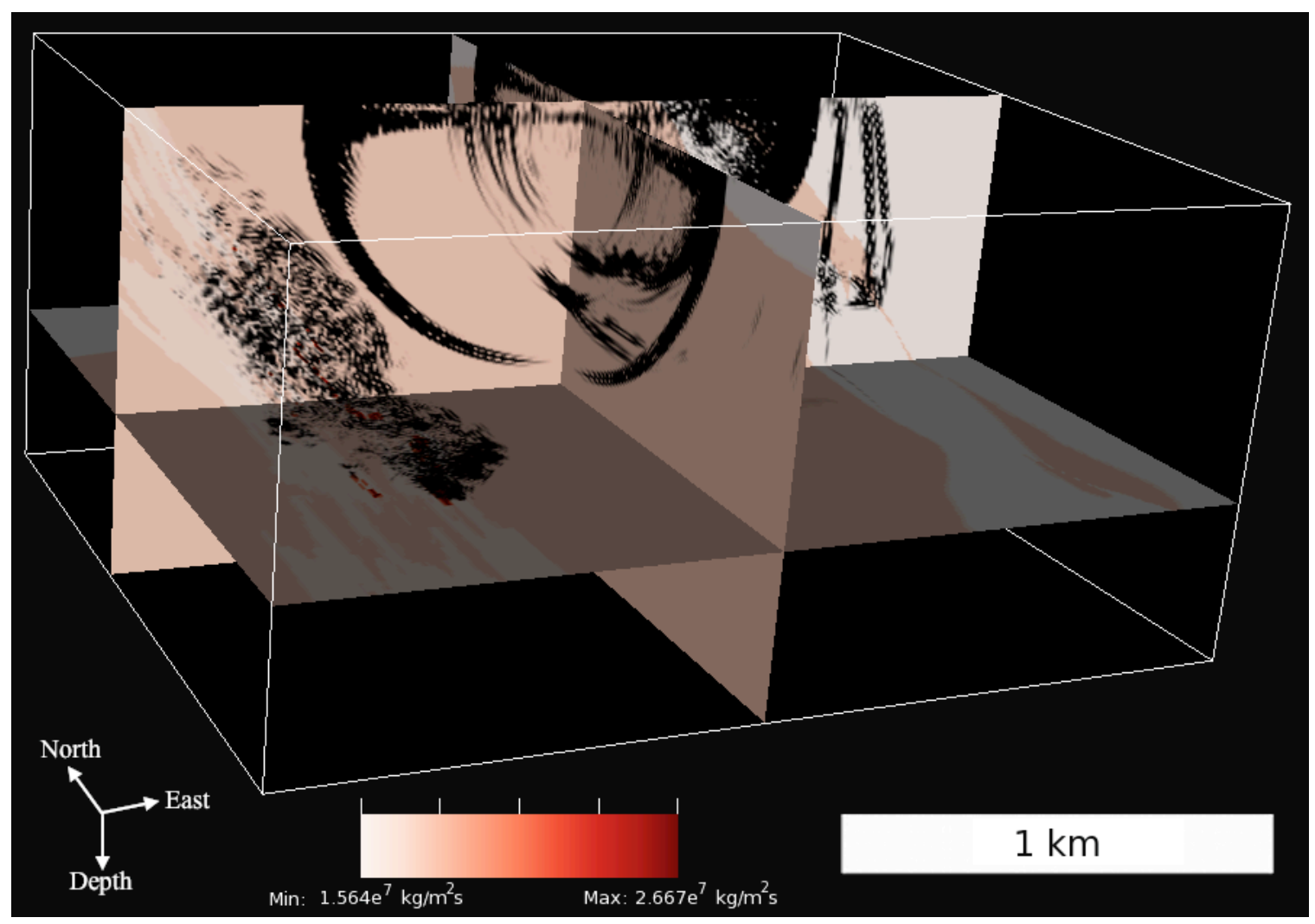

Figure 4.4.3 - 3D rotation wavefield visualization overlain on acoustic impedance from

the 'Lithological' model at $0.260 \mathrm{~s}$. The location of the source is approximated by the intersection of the vertical planes and the surface. The location of the receiver array is approximated by the vertical intersection of the two vertical planes above the horizontal plane. Darker colours indicate voxels with higher acoustic impedance. 


\section{5 - 'No Ore' model observations}

The vertical particle velocity shot gather from the final 'No Ore' model again shows the same downward-traveling reflections seen in the 'Ore/Missi' and 'Lithological' models. Also, three distinct reflection intervals with similar timing to those seen previously can be seen again, but only a single continuous and approximately linear event represents each. Interestingly, the late downward-traveling reflection seen in the 'Ore/Missi' and 'Lithological' models is not found in this shot gather. Some late reflections can also be seen, including one slightly curved and continuous reflection (white arrows in Figure 4.5.1) with an almost infinite apparent velocity. 


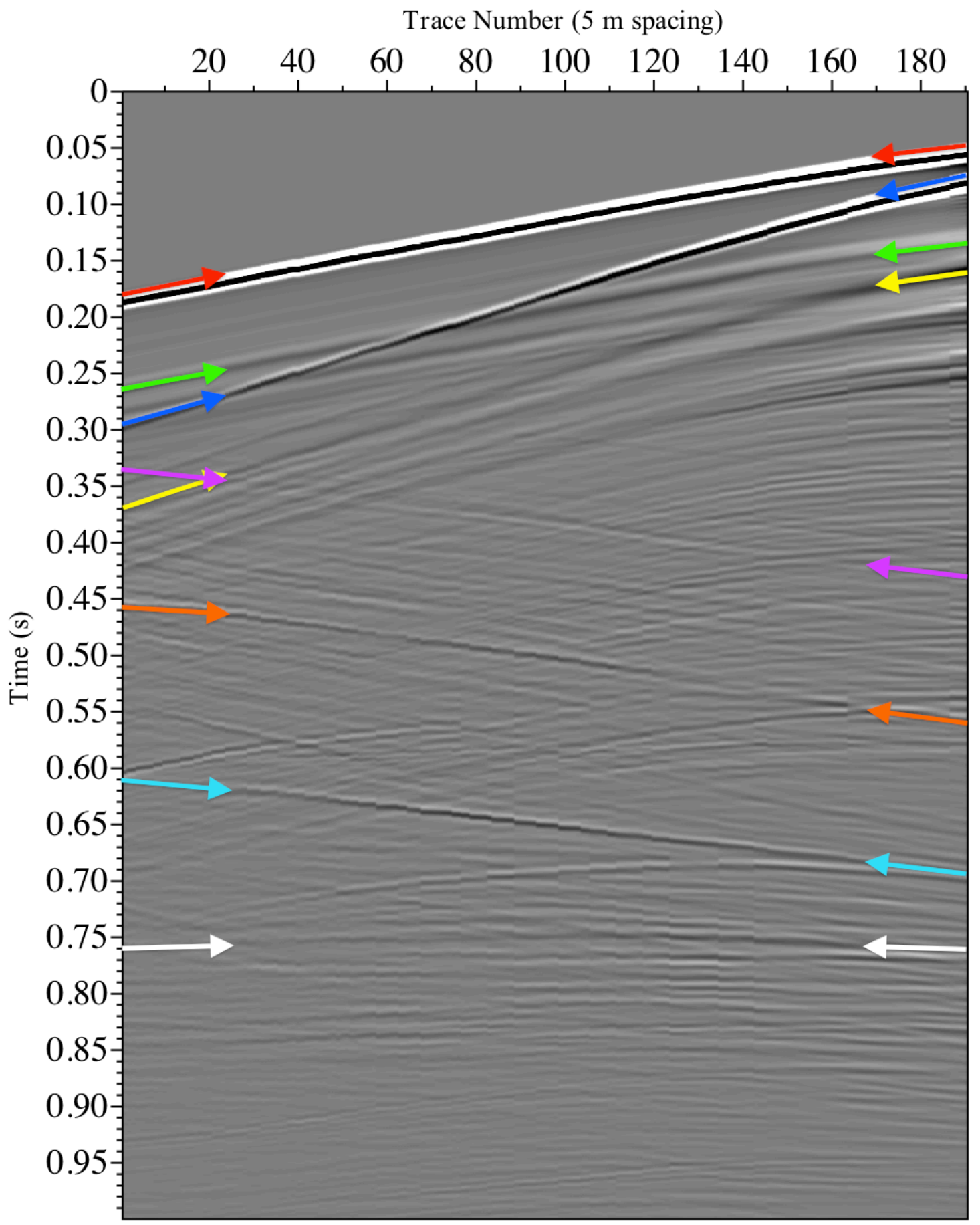

Figure 4.5.1 - Zero-offset vertical particle velocity synthetic VSP shot-gather from 4Q66W3 in the 'No Ore' model with prominent reflections identified by coloured arrows. 
Table 4.5.1 - Summary of annotated events in the vertical particle velocity shot gather from the 'No Ore' model shown in Figure 4.4.1.

\begin{tabular}{|c|c|c|c|c|c|c|c|}
\hline \begin{tabular}{|l} 
Model \\
Data set \\
Figure \\
\end{tabular} & $\begin{array}{l}\text { 'No Ore' } \\
\text { Vertical particle vel } \\
4.5 .1\end{array}$ & locity & & & & & \\
\hline Arrow Colour & Direct / Reflected & Alignment & Continuity & $\begin{array}{l}\text { Trace } 1 \\
\text { (s) }\end{array}$ & $\begin{array}{c}\text { Trace } 190 \\
\text { (s) }\end{array}$ & $\begin{array}{c}\text { Apparent velocity } \\
(\mathrm{m} / \mathrm{s})\end{array}$ & Notes \\
\hline Red & Direct P-wave & slightly curved & continuous & 0.180 & 0.042 & -6180 & \\
\hline Blue & Direct S-wave & curved & continuous & 0.298 & 0.074 & -3800 & weaker with depth \\
\hline Green & Reflection & curved & continuous & 0.262 & 0.132 & -6560 & several peaks \\
\hline Purple & Reflection & linear & continuous & 0.335 & 0.430 & 8970 & \\
\hline Yellow & Reflection & curved & continuous & 0.370 & 0.158 & -4020 & several peaks \\
\hline Orange & Reflection & linear & continuous & 0.459 & 0.560 & 8440 & \\
\hline Light blue & Reflection & linear & continuous & 0.610 & 0.697 & 9790 & \\
\hline White & Reflection & slightly curved & continuous & 0.758 & 0.763 & 170430 & \\
\hline
\end{tabular}




\section{0 - Discussion and comparison of data sets}

By comparing the shot gathers and 3D wavefield visualizations from the multiple 'Binary' model versions, the origins of some reflections can be determined. Events in the horizontal component data are typically weaker than in the vertical component data so for simplicity, only the vertical component particle velocity shot gathers will be compared here. In the data from the full 'Binary' model, three distinct intervals of reflections which originate at the ore lens contacts are displayed. The first reflection (green arrows in Figure 4.2.6) matches the timing and apparent velocity of the first reflection (green arrows in Figure 4.2.8) in the 'Binary (Callinan East)' data, and therefore originates at those contacts. The next reflection in the 'Binary' data (yellow arrows in Figure 4.2.6) matches the timing and apparent velocity of the first reflection (green arrows in Figure 4.2.7) in the 'Binary (Callinan North)' data, narrowing its point of origin. These first two reflections are relatively weak and are interpreted to be P-P reflections, due to their timing and apparent velocity.

The next traceable reflection (purple arrows in Figure 4.2.6) in the 'Binary' data matches another reflection (yellow arrows in Figure 4.2.8) in the 'Binary (Callinan East)' data. Another reflection (orange arrows in Figure 4.2.6) in the 'Binary' shot gather matches one (purple arrows in Figure 4.2.7) of the 'Binary (Callinan North)' data. These two are interpreted to be P-S converted waves due to their presence in the rotation shot gather (Figure 4.2.3), their timing and apparent velocity, are the strongest recorded events 
observed in the 'Binary' model and are therefore the most likely to be observed in the field data with much lower signal-to-noise ratios. The final traceable reflection (light blue arrows in Figure 4.2.6) in the 'Binary' model is interpreted as a mix of two reflections; one (orange arrows in Figure 4.2.7) from the 'Binary (Callinan North)' model and one (orange arrows in Figure 4.2.8) from the 'Binary (Callinan East)' model. These waves are interpreted to be S-S reflections, based on their presence in the rotation shot gather (Figure 4.2.3), their timing and their apparent velocity. Figure 5.1 displays the annotated events in the 'Binary', 'Binary (Callinan North)' and 'Binary (Callinan East)' data sets. Very little reflectivity is seen in data from the 'Binary (Callinan South)' or the 'Binary (777)' models. This is attributed to their position and dip not providing a large enough surface perpendicular to the source and receivers to produce a strong reflection. 


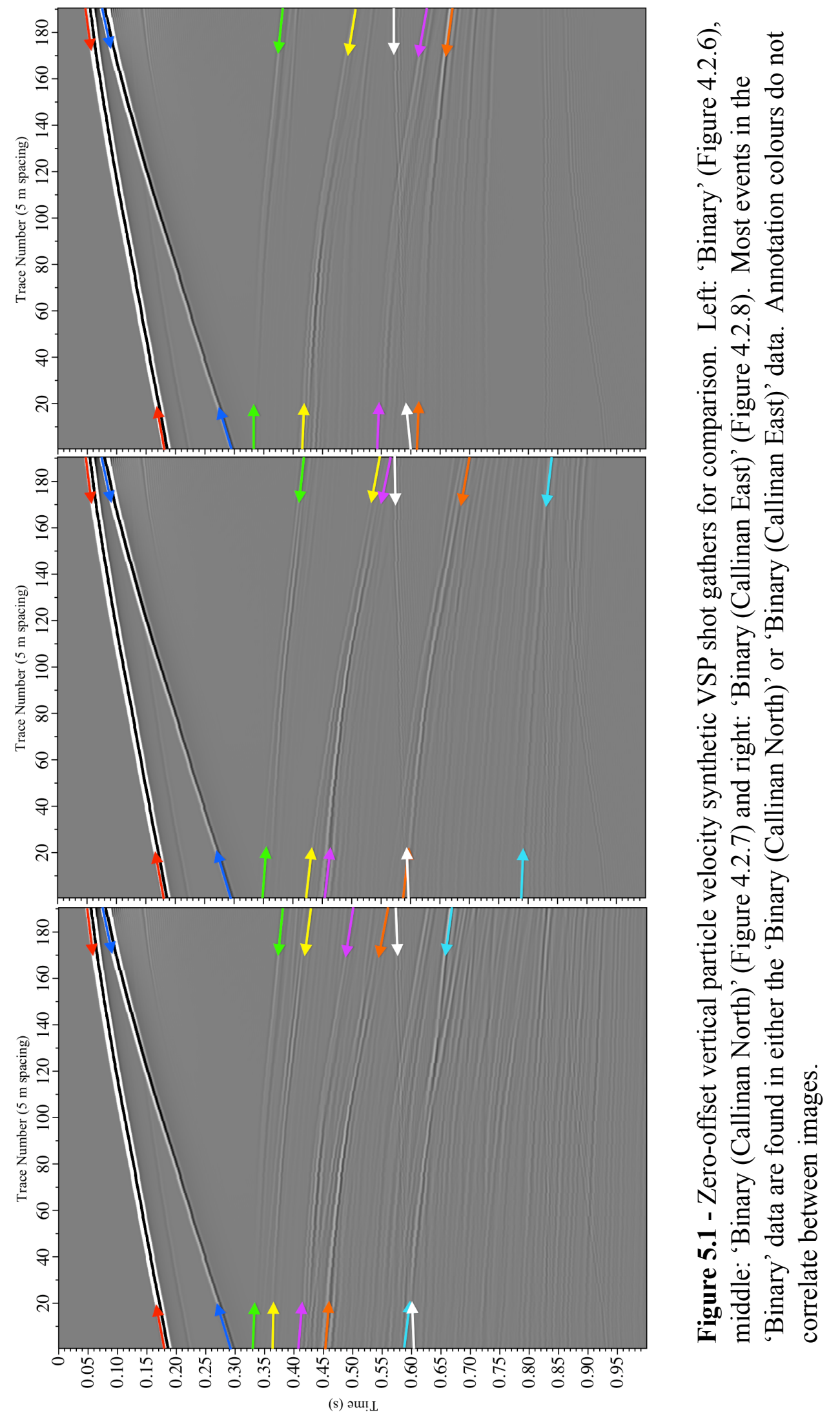


Comparison of the reflections in the 'Binary' and the 'Ore/Missi' shot gathers highlights the effect of the Missi metasedimentary basin in the shot gathers. Reverberatory downgoing waves following the direct P- and S- waves (green and yellow arrows in Figure 4.3.1) in the 'Ore/Missi' model are notably absent in the 'Binary' model. These waves travel downward through the receiver array and are interpreted to be the result of surface waves reflecting downwards from the contact between the Hidden formation basalt, which hosts the source, and the Missi metasedimentary basin. Reflections from the 'Binary' model between 0.30 and $0.60 \mathrm{~s}$ appear very similar, but another new event (white arrows in Figure 4.3.1) is now found. This event is an upward reflection from the Club Lake thrust fault to the North of the source location, which also separates the Missi group from the meta-basalt. The final annotated reflection in the 'Ore/Missi' data (dark green arrows in Figure 4.3.1) is not seen in the 'Binary' data so must be due to the basin's presence.

In the 'Lithological' model data, there appear to be several new weak events mixed with the reflections observed between 0.30 and $0.60 \mathrm{~s}$ in the 'Binary' and 'Ore/Missi' models. No new major reflections are seen, which indicates that all of the strongest reflections in the modeled data originate at the ore lenses and the Missi basin. This interpretation agrees with the fact that these contacts have the largest contrasts of acoustic impedance in the model, a result of the input physical properties. 
In the 'No Ore' model data, there is a considerable reduction in the number of reflections seen between 0.30 and $0.60 \mathrm{~s}$. A single weak reflection (purple arrows in Figure 4.5.1) approximately coincides with a group of previously-observed reflections (near the purple arrows in Figure 4.4.1). This event likely represents the ore-hosting contact between rhyolite and meta-basalt of the Callinan East and Callinan North lenses, which produced similar reflections. Similarly, a somewhat stronger single reflection (orange arrows in Figure 4.5.1) has similar timing and apparent velocity to another previously-mentioned group of reflections (near the orange arrows in Figure 4.4.1), perhaps representing a mode-converted P-S wave from the same horizon. The third relatively strong reflection (light blue arrows in Figure 4.5.1) from the Club Lake thrust fault which was seen in the 'Ore/Missi' model is seen again. Surprisingly, the last event found in the 'Ore/Missi' model (dark green arrows in Figure 4.3.1) is not found in the 'No Ore' model data. This means that this reflection must be related to ore lens reflections. A potential explanation would be upward-traveling ore lens reflections (i.e. orange and light blue arrows in Figure 4.3.1) are reflected back downward through the receiver array again from the contact with the Missi basin.

With all of the results of these models considered, reflections originating at ore lenses have been found to occur as intervals of mixed P- and S-waves, the strongest of which originate from the Callinan East and Callinan North lens groups, between 0.30 and $0.60 \mathrm{~s}$. These reflections were seen in many shot gathers from each of the models containing ore (e.g. Figure 4.2.6, 4.3.1 and 4.4.1). This result gives a strong indication that the geometry 
of the field VSP survey and the acoustic impedance contrasts in the surveyed volume will potentially produce an ore lens signature in the data. With these modeling observations and interpretations in mind, we can now attempt to correlate some reflections between the modeled and field VSP data.

The data from the field VSP survey is considerably more difficult to interpret due to high noise levels and discontinuous reflections. The first annotated reflection in the vertical component shot gather (green arrows in Figure 3.4.1), which intersects the borehole, is consistent with one observed in a previous study (White et al., in press.) and is thought to originate at an $\sim 20 \mathrm{~m}$ thick rhyolite interval present at this depth. The next annotated event in the vertical component data (red arrows in Figure 3.4.1) is not annotated in shot gathers from either of the horizontal components. The timing and apparent velocity of this event closely matches an event present in many of the models (green arrows in Figure 4.2.6 and purple arrows in Figure 4.4.1) which is interpreted in those models to originate from either the Callinan East or Callinan North ore lenses. Between 0.382 and $0.573 \mathrm{~s}$ in the vertical component shot gather another two events (blue and yellow arrows in Figure 3.4.1) are similar in continuity, timing and apparent velocity to reflections found in several of the models (purple and orange arrows in Figure 4.2.6 and orange arrows in Figure 4.4.1). In the models, this event is also interpreted to be produced at contacts between the host rock and the Callinan East or Callinan North ore lenses. Short intervals of reflectivity are found in similar areas of the horizontal component field shot gathers, but no comparable reflections have been annotated. Another reflection is found 
in all three components of the field VSP data (purple arrows in Figure 3.4.1, blue arrows in Figure 3.4.2 and yellow arrows in Figure 3.4.3). This event doesn't match any of the annotated reflections from the models. A final event, seen in all three components of the field data (orange arrows in Figure 3.4.1, purple arrows in Figure 3.4.2 and orange arrows in Figure 3.4.3) is matched to another event in the modeled data (light blue arrows in Figure 4.2.6 and light blue and white arrows in Figure 4.4.1). In the models, these reflections originate at the Callinan North and Callinan East groups of ore lenses and potentially from the Club Lake thrust fault. Figure 5.2 displays the vertical particle velocity data from the 'Lithological' model and the zero-offset vertical and transverse component field data for comparison. 

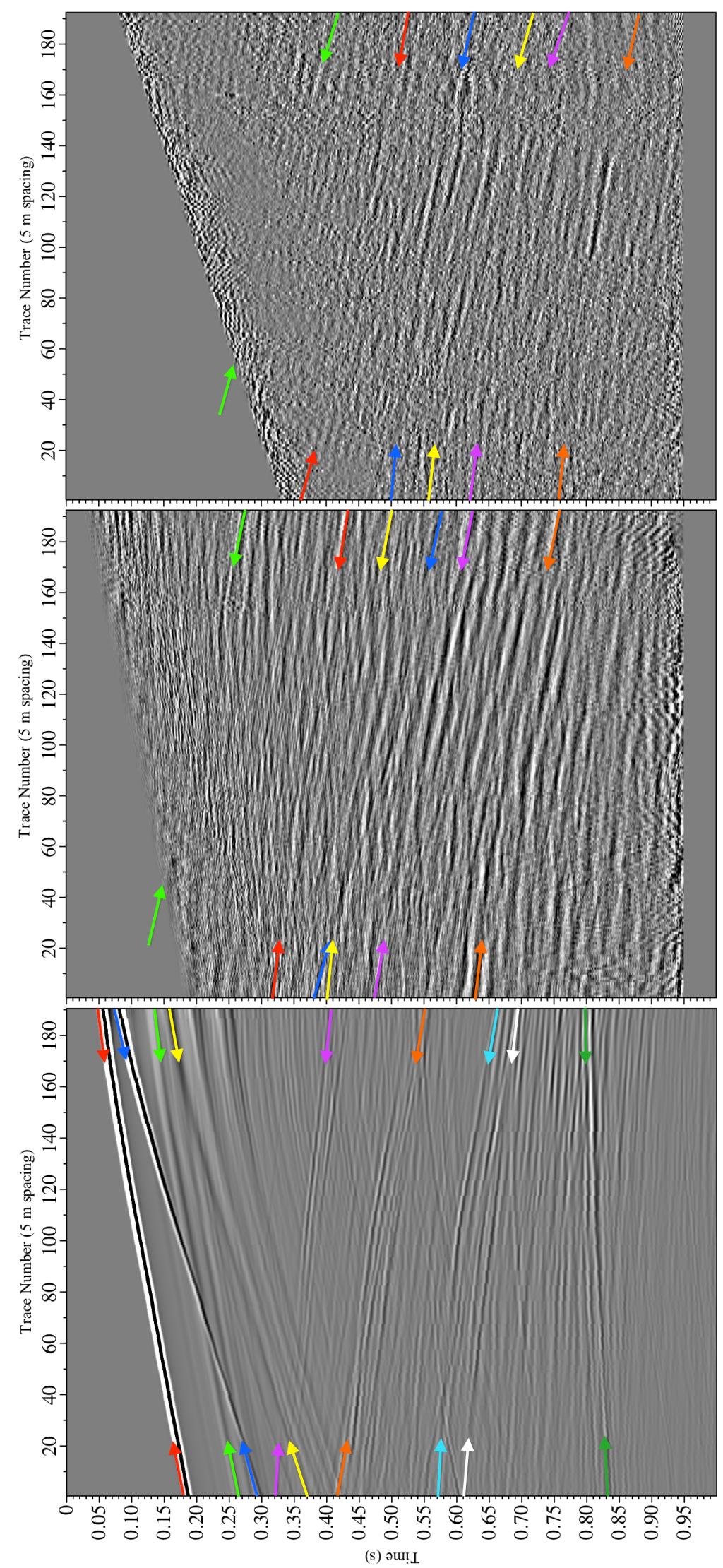

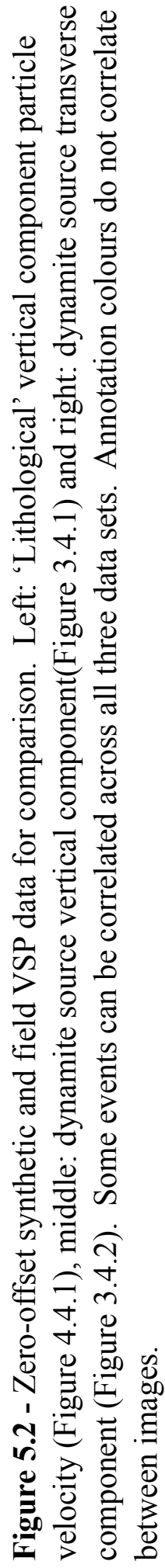


It is interesting to note that the strongest effects of the Missi basin in the modeled data are not seen in the field data. The early downward-traveling reflections (green and yellow arrows in Figure 4.4.1) may have been inadvertently removed during data processing, since they would have appeared similar to the direct P- and S-waves in the shot gathers and $f-k$ spectra. A much lower signal-to-noise ratio and the relatively weaker strength of the ore lens reflections may be responsible for the missing late downgoing reflection, seen in some modeled data (dark green arrows in Figure 4.4.1). 


\section{0 - Conclusions}

Seismic data from crystalline and active mining environments are difficult to interpret due to complex 3D geology and potential background noise. In this project, acquisition and processing of the 3-component zero-offset dynamite field VSP data from borehole 4Q66W3 in the Flin Flon mining camp has revealed a complex pattern of reflectivity. Analogous shot gathers from 3D finite difference modeling were found to be directly comparable with the field shot gathers by observing the timing and velocity of the direct waves. The comparisons of several models with increasing geological complexity and 3D wavefield visualizations allowed the determination of the origins of some reflections.

Some reflections originating from the up to $\sim 1200 \mathrm{~m}$ long VMS lenses in the modeled data were similar in shape, timing and apparent velocity to observed events in the field VSP data. These reflections were discontinuous to semi-continuous or mixed and had very high apparent velocities due to their offset points of origin. Most of these reflections also appear to be either scattered (P-P) or mode-converted (P-S) from the direct P-wave, based on their relative timing and apparent velocities. All interpreted ore lens reflections in the field VSP data are believed to originate at the Callinan North or Callinan East groups of ore lenses. It should be noted that these lenses were mostly mined out at the time of this survey, but their geometry is preserved and the backfilled material would provide a similar contrast in acoustic impedance with the host meta-basalt to that of the ore. The modeled ore lenses have an acoustic impedance approximately 1.5 times greater 
than any of the host geological units. The Callinan South and 777 lens groups were either hidden by other groups or dip away from the source and receiver array, not allowing the receivers to detect a strong reflection signal. Some other reflections in the modeled data, including the strongest events originating from the Missi metasedimentary basin, were not observed in the field VSP data, perhaps due to data-processing or variability in physical properties of some rock units.

In this study, the vertical component field and vertical particle velocity modeled data was clearer and more useful than the horizontal component field and particle velocity modeled data. However, the horizontal component field data sets have also proven to contain useful data including reflections originating at the ore lenses. From the modeled data, it appears that reflections are generally weaker in the horizontal components than in the vertical, but mode-converted reflections from the ore lenses are more likely to be captured in them than from other geological contacts. Weaker reflections in any data set will be more susceptible to overprinting by noise. Perhaps in data with a higher signalto-noise ratio there could be more valuable observations made in the horizontal component shot gathers, particularly in VSP surveys in crystalline environments with complex geology and deviated boreholes. Also, the Vibroseis source data was seen to have much lower signal-to-noise ratios, which may be due to the low source energy provided by relatively small vibrators. 
VSP surveys are not yet widely-used in mineral exploration applications. This study shows that the method has the potential to image VMS lenses in a greenstone host assemblage. The environmental challenges of cultural and electrical noise from an active mine, low source signal strength for the Vibroseis sources and poor coupling of the geophone tool led to a low overall signal-to-noise ratio. In this case, the VSP response from the ore lenses is not as prominent as initially anticipated. However, reflections originating from some of the VMS lenses were interpreted and described in each component of the zero-offset dynamite source VSP data. Also, 4Q66W3 passes $\sim 500 \mathrm{~m}$ from the nearest known ore lens, giving an idea of the effective range of this technique in this environment. With the significant investment required for drilling deep targets, it is important to extract as much geological information from boreholes as possible. VSP can provide a means to image dense bodies in the subsurface, even in challenging seismic environments. 


\subsection{References}

Beaty, K.S., Perron, G., Kay, I. and Adam, E. (2002) DSISoft - A MATLAB VSP data processing package. Computers \& Geosciences 28, pp 501-511.

Bellefleur, G., Müller, C., Snyder, D. and Matthews, L. (2004) Downhole seismic imaging of a massive sulfide orebody with mode-converted waves, Halfmile Lake, New Brunswick, Canada. Geophysics 69(2), pp 318-329.

Bohlen, T. (2002) Parallel 3-D viscoelastic finite difference seismic modelling. Computers \& Geosciences 28, pp 887-899.

Bohlen, T., Müller, C. and Milkereit, B. (2003) Elastic seismic-wave scattering from massive sulfide orebodies: on the role of composition and shape. In: Eaton, D., Milkereit, B. and Salisbury, M.H., Ed., Hardrock seismic exploration, SEG, Tulsa OK, pp 70-89.

Bohlen, T., De Nil, D., Köhn, D. and Jetschny, S. (2011) SOFI3D - seismic modeling with finite differences $3 D$ - acoustic and viscoelastic version: users guide. Department of Physics, Geophysical Institute. Karlsruhe Institute of Technology, Karlsruhe. 
Byron, J., Schetselaar, E., Gibson, H., Pehrsson, S., Lafrance, B., Devine, C. and Ames, D. (2010) Metal zoning within the Flin Flon-Callinan-777 ore system: a summary of preliminary results. Saskatchewan Open House 2010. Saskatchewan Geological Survey.

Caumon, G., Lepage, F., Sword, C.H. and Mallet, J.L. (2004) Building and editing a sealed geological model. Mathematical Geology 36(4), pp 405-424.

Corrigan, D., Pehrsson, S., Wodicka, N. and de Kemp, E. (2009) The Paleoproterozoic Trans-Hudson Orogen: a prototype of modern accretionary processes. In: Murphy, J.B., Keppie, J.D. and Hynes, A.J., Ed., Ancient orogens and modern analogues, Geological Society, London, Special Publications 327, pp 457-479.

de Kemp, E. (2000) 3-D visualisation of structural field data: examples from the Archean Coapatina Formation, Abitibi greenstone belt, Québec, Canada. Computers \& Geosciences 26, pp 509-530.

Devine, C. (2003) Origin and Emplacement of volcanogenic massive sulphide-hosting Paleoproterozoic volcaniclastic and effusive rocks within the Flin Flon subsidence structure, Manitoba and Saskatchewan, Canada. Laurentian University, Sudbury, 279 pp 
Dieteker, B. and White, D.J. (2007) Flin Flon, Manitoba vertical seismic profile (VSP) survey 2006/2007: first processing of VSP near offset dynamite data. Internal Report: Seismology and Electromagnetism Section, Geological Survey of Canada, Ottawa, $216 \mathrm{pp}$.

Eaton, D., Guest, S., Milkereit, B., Bleeker, W., Crick, D., Schmitt, D. and Salisbury, M. (1996) Seismic imaging of massive sulfide deposits: Part III. Borehole seismic imaging of near-vertical structures. Economic Geology 91, pp 835-840.

Fessenden, R.A. (1917) Method and apparatus for locating ore-bodies. United States Patent $\# 1,240,328$.

Galley, A.G., Syme, E.C. and Bailes, A.H. (2007) Metallogeny of the Paleoproterozoic Flin Flon belt, Manitoba and Saskatchewan. In: Goodfellow, W.D., Ed., mineral deposits of canada: a synthesis of major deposit types, district metallogeny, the evolution of geological provinces and exploration methods. Geological Association of Canada, Mineral Deposits division, Special Publication 5, pp 509-531.

Gal'perin, E.I. (1974) Vertical seismic profiling. In: White, J.E., Ed., Society of Exploration Geophysicists Special Publication 12, Tulsa OK, 270 pp. 
Gal'perin, E.I. (1977) Polyarizatsionnyi metod seismicheskikh issledovanii (Polarization method of seismic prospecting). Nedra Press, Moscow, 280 pp.

Gibson, H.L., DeWolfe, Y.M., Bailey, K., Devine, C., Lafrance, B., Gilmore, K., Simms, D. and Bailes, A.H. (2005) The Flin Flon caldera: ore localization within a Paleoproterozoic synvolcanic subsidence structure defined through mapping. Manitoba Industry, Economic Development and Mines, Manitoba Mining and Minerals Convention 2005, Winnipeg, pp 49.

Gibson, H.L., Lafrance, B., DeWolfe, M., Devine, C., Gilmore, K., Pehrsson, S. and Simard R-L. (2009) Volcanic reconstruction and post depositional modification of a cauldron subsidence structure within the Flin Flon VMS district. In: Program with Abstracts, Prospectors and Developers Association of Canada, Annual Meeting, Toronto.

Green, A.G. and Mair, J.A. (1983) Subhorizontal fractures in a granitic pluton: their detection and implications for radioactive waste disposal. Geophysics 48(11), pp 1428-1449.

Hardage, B.A. (2000) Vertical seismic profiling: principles. Elsevier Science, Oxford, $552 \mathrm{pp}$. 
Hinds, R.C., Anderson, N.L. and Kuzmiski, R.D. (1996) VSP interpretive processing: theory and practice. Society of Exploration Geophysicists Open File Publications 3, Tulsa OK, 205 pp.

Hodgetts, D. and Howell, J.A. (2000) Synthetic seismic modelling of a large-scale geological cross-section from the Book Cliffs, Utah, USA. Petroleum Geoscience 6, pp 221-229.

Hoffman, P. (1988) United plates of America, the birth of a craton: early Proterozoic assembly and growth of Laurentia. Annual Review of Earth and Planetary Sciences 16, pp 543-603.

Jolly, R.N. (1953) Deep-hole geophone study in Garvin County, Oklahoma. Geophysics 18, pp 662-670.

Kelly, K.R., Alford, R.M. and Whitmore, N.D. (1982) Modeling - the forward method. In: Jain, K.C. and Figueiredo, R.J.P., Ed., Concepts and Techniques in Oil and Gas Exploration, Society of Exploration Geophysicists, Tulsa OK, 289 pp.

Kennett, P., Ireson, R.L. and Conn, P.J. (1980) Vertical seismic profiles - their applications in exploration geophysics. Geophysical Prospecting 28, pp 676-699. 
Komatitsch, D. and Martin, R. (2007) An unsplit convolutional perfectly matched layer technique improved at grazing incidence for the viscoelastic wave equation. Geophysics 72(5), pp 155-157.

LaJaunie, C., Courrioux, G. and Manuel, L. (1997) Foliation fields and 3D cartography in geology: principles of a method based on potential interpolation. Mathematical Geology 29(4), pp 571-584.

Levin, F.K. and Lynn, R.D. (1958) Deep hole geophone studies. Geophysics 23, pp 639-664.

Lewry, J.F. and Collerson, K.D. (1990) The Trans-Hudson Orogen: extent, subdivisions and problems. In: Lewry, J.F. and Stauffer, M.R., Ed., The early Proterozoic Trans-Hudson Orogen of North America, Geological Association of Canada Special Paper 37, pp 114.

Malinowski, M., Schetselaar, E. and White, D.J. (2012) 3D seismic imaging of volcanogenic massive sulfide deposits in the Flin Flon mining camp, Canada: part 2 - forward modeling. Geophysics 77(5), pp WC81-WC93. 
McCollum, B. and LaRue, W.W. (1931) Utilization of existing wells in seismograph work. Early Geophysical Papers 1, pp 119-127 (Also Bulletin of the American Association of Petroleum Geologists 15, pp 1409-1417).

Miao, X-G., Moon, W.M. and Milkereit, B. (1995) A multioffset, three-component VSP study in the Sudbury Basin, Geophysics 60(2), pp 341-353.

Perron, G., Eaton, D.W., Elliot, B. and Schmitt, D. (2003) Application of downhole seismic imaging to map near-vertical structures: Normétal (Abitibi greenstone belt), Québec. In: Easton, D.W., Milkereit, B. and Salisbury, M.H., Ed., Hardrock seismic exploration, SEG, Tulsa OK, pp 194-206.

Pretorius, C.C., Jamison, A.A. and Irons, C. (1989) Seismic exploration in the Witwatersrand Basin, Republic of South Africa. Proceedings Exploration 87, third decennial international conference on geophysics and geochemical exploration for minerals and groundwater, Ontario Geological Survey, Special Publication 3, pp 241-253.

Riggs, E.D. (1955) Seismic wave types in a borehole. Geophysics 20, pp 53-67. 
Salisbury, M.H., Harvey, C.W. and Matthews, L. (2003) The acoustic properties of ores and host rocks in hardrock terranes. In: Eaton, D.W., Milkereit, B. and Salisbury, M.H., Ed., Hardrock seismic exploration, SEG, Tulsa OK, pp 9-19.

Schetselaar, E., Pehrsson, S., Devine, C., Currie, M., White, D.J. and Malinowski, M. (2010) The Flin Flon 3D Knowledge Cube. Geological Survey of Canada Open File 6313,35 pp.

Schmitt, D.R. (2006) Seismic acquisition in support of hard rock mine exploration, Flin Flon, Manitoba: a collaboration between the experimental geophysics group at the U of Alberta and the Geological Survey of Canada. Internal Report: Institute for Geophysical Research, University of Alberta, Edmonton, 8 pp.

Simard, R-L., MacLachlan, K., Gibson, H.L., DeWolfe, Y.M., Devine, C., Kremer, P.D., Lafrance, B., Ames, D.E., Syme, E.C., Bailes, A.H., Bailey, K., Price, D., Pehrsson, S., Cole, E., Lewis, D. and Galley, A.G. (2010) Geology of the Flin Flon area, Manitoba and Saskatchewan (part of NTS 63K12, 13). Manitoba Innovation, Energy and Mines, Manitoba Geological Survey Map 2010-1 and Saskatchewan Ministry of Energy and Resources, Geoscience Map 2010-2, 1 colour map, scale 1:10 000. 
Snyder, D., Perron, G., Pflug, K. and Stevens, K. (2002) New insights into the structure of the Sudbury Igneous Complex from downhole seismic studies. Canadian Journal of Earth Sciences 39, pp 943-951.

Stern, R.A., Machado, N., Syme, E.C., Lucas, S.B. and David, J. (1999) Chronology of crustal growth and recycling in the Paleoproterozoic Amisk collage (Flin Flon belt), Trans-Hudson Orogen, Canada. Canadian Journal of Earth Sciences 36, pp 1807-1827.

Syme, E.C. and Bailes, A.H. (1993) Stratigraphic and tectonic setting of Early Proterozoic volcanogenic massive sulphide deposits, Flin Flon, Manitoba. Economic Geology 88(3), pp 566-589.

Temme, P. and Müller, G. (1982) Numerical simulation of vertical seismic profiling. Journal of Geophysics 50, pp 177-188.

Tessier, A.C. and O’Donnell, J.J. (2001) Callinan / 777 deposit: compilation and target generation project. Internal Report: Hudson Bay Mining and Smelting Co, A.C. Tessier Consulting Geologist, Kingston, 154 pp. 
White, D.J., Secord, D. and Malinowski, M. (2012) 3D seismic imaging of volcanogenic massive sulfide deposits in the Flin Flon mining camp, Canada: Part 1 - seismic results. Geophysics 77(5), pp WC47-WC58.

White, D.J., Mwenifumbo, J. and Salisbury, M.H. (in press) Seismic properties of rocks from the Flin Flon VMS camp. Submitted to Economic Geology, 36 pp.

Wyatt, K.D. (1980) Synthetic vertical seismic profile. Geophysics 46(6), pp 880-891. 


\title{
Appendix A - Processing operations and parameters for all field VSP data collected
}

\section{Appendix A.1 - Globe Claritas processing operations and parameters used to processes}

\author{
zero-offset dynamite source VSP data from 4Q66W3.
}

\begin{tabular}{|c|c|c|c|}
\hline $\begin{array}{l}\text { Borehole } \\
\text { Data Set } \\
\text { Component }\end{array}$ & $\begin{array}{l}\text { 4Q66W3 } \\
\text { Zero-offset dynamite } \\
\text { Vertical }\end{array}$ & & \\
\hline PROCESSING STEP & & TOOL & INPUT PARAMETERS \\
\hline \multicolumn{2}{|c|}{ Add first break pick times into trace header } & ADDPICK & Pick file, Trace header \\
\hline Spiking Wiener decon & olution & DECONW & Flattened to P-wave picks, $100 \mathrm{~ms}$ operator, $0.1 \%$ pre-whitening \\
\hline \multirow{2}{*}{\multicolumn{2}{|c|}{$\begin{array}{l}\text { Frequency-domain time and spatially varying filter } \\
\text { Frequency-domain time and spatially varying filter }\end{array}$}} & FDFILT & Bandstop: $57-59 \mathrm{~Hz}$ tapered high cut, $61-63 \mathrm{~Hz}$ tapered low cut \\
\hline & & FDFILT & Bandstop: $175-179 \mathrm{~Hz}$ tapered high cut, $181-185 \mathrm{~Hz}$ tapered low cut \\
\hline \multirow{2}{*}{\multicolumn{2}{|c|}{$\begin{array}{l}\text { Frequency-domain time and spatially varying filter } \\
\text { Generalized muting of } f-k \text { spectra }\end{array}$}} & FDFILT & Bandpass: $10-20 \mathrm{~Hz}$ tapered low cut, $270-300 \mathrm{~Hz}$ tapered high cut \\
\hline & & FKMUTE & Flattened to P-wave picks, custom direct $\mathrm{P}$-wave mute file \\
\hline \multicolumn{2}{|c|}{ Generalized muting of $f-k$ spectra } & FKMUTE & Custom direct $\mathrm{P}$-wave mute file \\
\hline \multicolumn{2}{|c|}{ Generalized muting of $f-k$ spectra } & FKMUTE & Custom aliased P-wave mute file \\
\hline \multicolumn{2}{|c|}{ Generalized muting of $f-k$ spectra } & FKMUTE & Custom direct S-wave mute file \\
\hline \multicolumn{2}{|l|}{ Trace balance } & BALANCE & Full trace amplitude balance \\
\hline \multicolumn{2}{|c|}{ FX-domain complex weiner deconvolution } & FX DECON & 12-trace filter applied to 24 traces, $200 \mathrm{~ms}$ window \\
\hline \multicolumn{2}{|c|}{ Mutes to input trace header value } & ZEROMUTE & P-wave picks, $40 \mathrm{~ms}$ cosine taper \\
\hline \multicolumn{2}{|l|}{ Bulk static shift } & BULKSHIFT & $-50 \mathrm{~ms}$ \\
\hline \multicolumn{2}{|l|}{ Automatic gain control } & AGC & $250 \mathrm{~ms}$ Window \\
\hline
\end{tabular}

\begin{tabular}{|c|c|c|c|}
\hline $\begin{array}{l}\text { Borehole } \\
\text { Data Set } \\
\text { Component }\end{array}$ & $\begin{array}{l}\text { 4Q66W3 } \\
\text { Zero-offset dynamite } \\
\text { Transverse }\end{array}$ & & \\
\hline PROCESSING STEP & & TOOL & INPUT PARAMETERS \\
\hline \multirow{7}{*}{\multicolumn{2}{|c|}{$\begin{array}{l}\text { Add first break pick times into trace header } \\
\text { Automatic gain control } \\
\text { Generalized muting of seismic data } \\
\text { Frequency-domain time and spatially varying filter } \\
\text { Frequency-domain time and spatially varying filter } \\
\text { Frequency-domain time and spatially varying filter } \\
\text { Generalized muting of } f \text { - } k \text { spectra }\end{array}$}} & ADDPICK & Pick file, Trace header \\
\hline & & AGC & $250 \mathrm{~ms}$ Window \\
\hline & & MUTE & S-wave picks, $12 \mathrm{~ms}$ cosine taper \\
\hline & & FDFILT & Bandstop: $57-59 \mathrm{~Hz}$ tapered high cut, $61-63 \mathrm{~Hz}$ tapered low cut \\
\hline & & FDFILT & Bandstop: $175-179 \mathrm{~Hz}$ tapered high cut, $181-185 \mathrm{~Hz}$ tapered low cut \\
\hline & & FDFILT & Bandpass: $10-20 \mathrm{~Hz}$ tapered low cut, $270-300 \mathrm{~Hz}$ tapered high cut \\
\hline & & FKMUTE & Custom direct S-wave mute file \\
\hline \multicolumn{2}{|l|}{ Trace balance } & BALANCE & Full trace amplitude balance \\
\hline \multicolumn{2}{|c|}{ FX-domain complex weiner deconvolution } & FX DECON & 12-trace filter applied to 24 traces, $200 \mathrm{~ms}$ window \\
\hline \multicolumn{2}{|c|}{ Generalized muting of seismic data } & MUTE & S-wave picks, $12 \mathrm{~ms}$ cosine taper \\
\hline \multicolumn{2}{|l|}{ Bulk static shift } & BULKSHIFT & $-50 \mathrm{~ms}$ \\
\hline \multicolumn{2}{|l|}{ Automatic gain control } & AGC & $250 \mathrm{~ms}$ Window \\
\hline
\end{tabular}

\begin{tabular}{|c|c|c|c|}
\hline $\begin{array}{l}\text { Borehole } \\
\text { Data Set } \\
\text { Component } \\
\end{array}$ & \multicolumn{3}{|l|}{$\begin{array}{l}\text { 4Q66W3 } \\
\text { Zero-offset dynamite } \\
\text { Radial }\end{array}$} \\
\hline PROCESSING STEP & & TOOL & INPUT PARAMETERS \\
\hline \multicolumn{2}{|c|}{ Add first break pick times into trace header } & ADDPICK & Pick file, Trace header \\
\hline \multicolumn{2}{|l|}{ Automatic gain control } & AGC & $250 \mathrm{~ms}$ Window \\
\hline \multicolumn{2}{|c|}{ Generalized muting of seismic data } & MUTE & S-wave picks, $12 \mathrm{~ms}$ cosine taper \\
\hline \multicolumn{2}{|c|}{ Frequency-domain time and spatially varying filter } & FDFILT & Bandstop: $57-59 \mathrm{~Hz}$ tapered high cut, $61-63 \mathrm{~Hz}$ tapered low cut \\
\hline \multicolumn{2}{|c|}{ Frequency-domain time and spatially varying filter } & FDFILT & Bandstop: $175-179 \mathrm{~Hz}$ tapered high cut, $181-185 \mathrm{~Hz}$ tapered low cut \\
\hline \multirow{2}{*}{\multicolumn{2}{|c|}{$\begin{array}{l}\text { Frequency-domain time and spatially varying filter } \\
\text { Generalized muting of } f-k \text { spectra }\end{array}$}} & FDFILT & Bandpass: $10-20 \mathrm{~Hz}$ tapered low cut, $270-300 \mathrm{~Hz}$ tapered high cut \\
\hline & & FKMUTE & Custom direct $\mathrm{S}$-wave mute file \\
\hline \multicolumn{2}{|c|}{ Trace balance } & BALANCE & Full trace amplitude balance \\
\hline \multicolumn{2}{|c|}{ FX-domain complex weiner deconvolution } & FX DECON & 12-trace filter applied to 24 traces, $200 \mathrm{~ms}$ window \\
\hline \multicolumn{2}{|c|}{ Generalized muting of seismic data } & MUTE & S-wave picks, $12 \mathrm{~ms}$ cosine taper \\
\hline \multicolumn{2}{|l|}{ Bulk static shift } & BULKSHIFT & $-50 \mathrm{~ms}$ \\
\hline \multicolumn{2}{|l|}{ Automatic gain control } & AGC & $250 \mathrm{~ms}$ Window \\
\hline
\end{tabular}


Appendix A.2 - Globe Claritas processing operations and parameters used to processes

\section{far-offset dynamite source VSP data from 4Q66W3.}

\begin{tabular}{|c|c|c|c|}
\hline \begin{tabular}{|l|} 
Borehole \\
Data Set \\
Component \\
\end{tabular} & \begin{tabular}{|l|} 
4Q66W3 \\
Far-offset dynamite \\
Vertical
\end{tabular} & & \\
\hline PROCESSING STEP & & TOOL & INPUT PARAMETERS \\
\hline \multirow{6}{*}{\multicolumn{2}{|c|}{$\begin{array}{l}\text { Add first break pick times into trace header } \\
\text { Spiking Wiener deconvolution } \\
\text { Frequency-domain time and spatially varying filter } \\
\text { Frequency-domain time and spatially varying filter } \\
\text { Frequency-domain time and spatially varying filter }\end{array}$}} & ADDPICK & Pick file, Trace header \\
\hline & & DECONW & Flattened to P-wave picks, $100 \mathrm{~ms}$ operator, $0.1 \%$ pre-whitening \\
\hline & & FDFILT & Bandstop: $57-59 \mathrm{~Hz}$ tapered high cut, $61-63 \mathrm{~Hz}$ tapered low cut \\
\hline & & FDFILT & Bandstop: $175-179 \mathrm{~Hz}$ tapered high cut, $181-185 \mathrm{~Hz}$ tapered low cut \\
\hline & & FDFILT & Bandpass: $10-20 \mathrm{~Hz}$ tapered low cut, $270-300 \mathrm{~Hz}$ tapered high cut \\
\hline & & FKMUTE & Flattened to P-wave picks, custom direct P-wave mute file \\
\hline \multicolumn{2}{|c|}{$\begin{array}{l}\text { Generalized muting of } f-k \text { spectra } \\
\text { Generalized muting of } f-k \text { spectra }\end{array}$} & FKMUTE & Custom direct $\mathrm{P}$-wave mute file \\
\hline \multirow{2}{*}{\multicolumn{2}{|c|}{ Generalized muting of $f-k$ spectra }} & FKMUTE & Custom aliased P-wave mute file \\
\hline & & FKMUTE & Custom direct S-wave mute file \\
\hline \multicolumn{2}{|l|}{ Trace balance } & BALANCE & Full trace amplitude balance \\
\hline \multicolumn{2}{|c|}{ Mutes to input trace header value } & ZEROMUTE & P-wave picks, $40 \mathrm{~ms}$ cosine taper \\
\hline \multirow{2}{*}{\multicolumn{2}{|c|}{$\begin{array}{l}\text { Bulk static shift } \\
\text { Automatic gain control }\end{array}$}} & BULKSHIFT & $-50 \mathrm{~ms}$ \\
\hline & & AGC & $250 \mathrm{~ms}$ Window \\
\hline
\end{tabular}

\begin{tabular}{|c|c|c|c|}
\hline $\begin{array}{l}\text { Borehole } \\
\text { Data Set } \\
\text { Component }\end{array}$ & $\begin{array}{l}\text { 4Q66W3 } \\
\text { Far-offset dynamite } \\
\text { Transverse }\end{array}$ & & \\
\hline PROCESSING STEP & & TOOL & INPUT PARAMETERS \\
\hline \multirow{5}{*}{\multicolumn{2}{|c|}{$\begin{array}{l}\text { Add first break pick times into trace header } \\
\text { Automatic gain control } \\
\text { Mutes to input trace header value } \\
\text { Frequency-domain time and spatially varying filter } \\
\text { Frequency-domain time and spatially varying filter }\end{array}$}} & ADDPICK & Pick file, Trace header \\
\hline & & AGC & $250 \mathrm{~ms}$ Window \\
\hline & & ZEROMUTE & P-wave picks, $40 \mathrm{~ms}$ cosine taper \\
\hline & & FDFILT & Bandstop: $57-59 \mathrm{~Hz}$ tapered high cut, $61-63 \mathrm{~Hz}$ tapered low cut \\
\hline & & FDFILT & Bandstop: $175-179 \mathrm{~Hz}$ tapered high cut, $181-185 \mathrm{~Hz}$ tapered low cut \\
\hline \multirow{2}{*}{\multicolumn{2}{|c|}{$\begin{array}{l}\text { Frequency-domain time and spatially varying filter } \\
\text { Zero-phase frequency-domain spectral equalisation }\end{array}$}} & FDFILT & Bandpass: $10-20 \mathrm{~Hz}$ tapered low cut, $270-300 \mathrm{~Hz}$ tapered high cut \\
\hline & & SPEQ & $30 \mathrm{~Hz}$ frequency-domain AGC \\
\hline \multicolumn{2}{|c|}{ Generalized muting of $f-k$ spectra } & FKMUTE & Custom direct S-wave mute file \\
\hline \multicolumn{2}{|c|}{ Mutes to input trace header value } & ZEROMUTE & $\mathrm{P}$-wave picks, $40 \mathrm{~ms}$ cosine taper \\
\hline \multicolumn{2}{|l|}{ Trace balance } & BALANCE & Full trace amplitude balance \\
\hline \multicolumn{2}{|l|}{ Bulk static shift } & BULKSHIFT & $-50 \mathrm{~ms}$ \\
\hline \multicolumn{2}{|l|}{ Automatic gain control } & AGC & $250 \mathrm{~ms}$ Window \\
\hline
\end{tabular}

\begin{tabular}{|c|c|c|c|}
\hline $\begin{array}{l}\text { Borehole } \\
\text { Data Set } \\
\text { Component }\end{array}$ & \multicolumn{3}{|l|}{$\begin{array}{l}\text { 4Q66W3 } \\
\text { Far-offset dynamite } \\
\text { Radial }\end{array}$} \\
\hline PROCESSING STEP & & TOOL & INPUT PARAMETERS \\
\hline \multirow{3}{*}{\multicolumn{2}{|c|}{$\begin{array}{l}\text { Add first break pick times into trace header } \\
\text { Automatic gain control } \\
\text { Mutes to input trace header value }\end{array}$}} & ADDPICK & Pick file, Trace header \\
\hline & & AGC & $250 \mathrm{~ms}$ Window \\
\hline & & ZEROMUTE & P-wave picks, $40 \mathrm{~ms}$ cosine taper \\
\hline \multirow{2}{*}{\multicolumn{2}{|c|}{$\begin{array}{l}\text { Frequency-domain time and spatially varying filter } \\
\text { Frequency-domain time and spatially varying filter }\end{array}$}} & FDFILT & Bandstop: $57-59 \mathrm{~Hz}$ tapered high cut, $61-63 \mathrm{~Hz}$ tapered low cut \\
\hline & & FDFILT & Bandstop: $175-179 \mathrm{~Hz}$ tapered high cut, $181-185 \mathrm{~Hz}$ tapered low cut \\
\hline \multirow{2}{*}{\multicolumn{2}{|c|}{$\begin{array}{l}\text { Frequency-domain time and spatially varying filter } \\
\text { Zero-phase frequency-domain spectral equalisation }\end{array}$}} & FDFILT & Bandpass: $10-20 \mathrm{~Hz}$ tapered low cut, $270-300 \mathrm{~Hz}$ tapered high cut \\
\hline & & SPEQ & $30 \mathrm{~Hz}$ frequency-domain $\mathrm{AGC}$ \\
\hline \multirow{2}{*}{\multicolumn{2}{|c|}{$\begin{array}{l}\text { Generalized muting of } f-k \text { spectra } \\
\text { Mutes to input trace header value }\end{array}$}} & FKMUTE & Custom direct S-wave mute file \\
\hline & & ZEROMUTE & P-wave picks, $40 \mathrm{~ms}$ cosine taper \\
\hline \multicolumn{2}{|l|}{ Trace balance } & BALANCE & Full trace amplitude balance \\
\hline \multicolumn{2}{|l|}{ Bulk static shift } & BULKSHIFT & $-50 \mathrm{~ms}$ \\
\hline \multicolumn{2}{|l|}{ Automatic gain control } & AGC & $250 \mathrm{~ms}$ Window \\
\hline
\end{tabular}


Appendix A.3 - Globe Claritas processing operations and parameters used to processes

\section{zero-offset P Vibroseis source VSP data from 4Q66W3.}

\begin{tabular}{|c|c|c|c|}
\hline \begin{tabular}{|l} 
Borehole \\
Data Set \\
Component
\end{tabular} & \begin{tabular}{|l} 
4Q66W3 \\
Zero-offset P Vibroseis \\
Vertical
\end{tabular} & & \\
\hline PROCESSING STEP & & TOOL & INPUT PARAMETERS \\
\hline \multirow{5}{*}{\multicolumn{2}{|c|}{$\begin{array}{l}\text { Add first break pick times into trace header } \\
\text { Spiking Wiener deconvolution } \\
\text { Frequency-domain time and spatially varying filter } \\
\text { Frequency-domain time and spatially varying filter } \\
\text { Frequency-domain time and spatially varying filter }\end{array}$}} & ADDPICK & Pick file, Trace header \\
\hline & & DECONW & Flattened to $\mathrm{P}$-wave picks, $100 \mathrm{~ms}$ operator, $0.1 \%$ pre-whitening \\
\hline & & FDFILT & Bandstop: $57-59 \mathrm{~Hz}$ tapered high cut, $61-63 \mathrm{~Hz}$ tapered low cut \\
\hline & & FDFILT & Bandstop: $175-179 \mathrm{~Hz}$ tapered high cut, $181-185 \mathrm{~Hz}$ tapered low cut \\
\hline & & FDFILT & Bandpass: $15-25 \mathrm{~Hz}$ tapered low cut, $250-270 \mathrm{~Hz}$ tapered high cut \\
\hline \multirow{2}{*}{\multicolumn{2}{|c|}{$\begin{array}{l}\text { Zero-phase frequency-domain spectral equalisation } \\
\text { Generalized muting of } f-k \text { spectra }\end{array}$}} & SPEQ & $30 \mathrm{~Hz}$ frequency-domain AGC \\
\hline & & FKMUTE & Flattened to P-wave picks, custom direct P-wave mute file \\
\hline \multicolumn{2}{|c|}{ Generalized muting of $f-k$ spectra } & FKMUTE & Custom direct $\mathrm{P}$-wave mute file \\
\hline \multicolumn{2}{|c|}{ Generalized muting of $f-k$ spectra } & FKMUTE & Custom aliased P-wave mute file \\
\hline \multirow{2}{*}{\multicolumn{2}{|c|}{$\begin{array}{l}\text { Generalized muting of } f-k \text { spectra } \\
\text { FX-domain complex weiner deconvolution }\end{array}$}} & FKMUTE & Custom direct S-wave mute file \\
\hline & & FX DECON & 12-trace filter applied to 24 traces, $200 \mathrm{~ms}$ window \\
\hline \multicolumn{2}{|c|}{$\begin{array}{l}\text { Mutes to input trace header value } \\
\text { Automatic gain control }\end{array}$} & ZEROMUTE & P-wave picks, $40 \mathrm{~ms}$ cosine taper \\
\hline
\end{tabular}

\begin{tabular}{|l|l|}
\hline $\begin{array}{l}\text { Borehole } \\
\text { Data Set } \\
\text { Component }\end{array}$ & $\begin{array}{l}\text { 4Q66W3 } \\
\text { Zero-offset P Vibroseis } \\
\text { Transverse }\end{array}$ \\
\hline PROCESSING STEP & \\
\hline
\end{tabular}

\begin{tabular}{|l|l|}
\hline PROCESSING STEP & \\
\hline Add first break pick times into trace header
\end{tabular}

Add first break pick times into trace header

Automatic gain control

Generalized muting of seismic data

TOOL

INPUT PARAMETERS

Frequency-domain time and spatially varying filter FDFILT

Frequency-domain time and spatially varying filter FDFILT

Frequency-domain time and spatially varying filter FDFILT

Zero-phase frequency-domain spectral equalisation SPEQ

Generalized muting of $\boldsymbol{f}-\boldsymbol{k}$ spectra

Trace balance

FKMUTE

Generalized muting of seismic data

BALANCE

Automatic gain control

\begin{tabular}{|l|l|}
\hline Borehole & $4 \mathrm{Q} 66 \mathrm{~W} 3$ \\
Data Set & Zero-offset P Vibroseis \\
Component & Radial \\
\hline PROCESSING STEP & \\
\hline
\end{tabular}

MUTE

AGC

Pick file, Trace header

$250 \mathrm{~ms}$ Window

$\mathrm{S}$-wave picks, $12 \mathrm{~ms}$ cosine taper

Bandstop: $57-59 \mathrm{~Hz}$ tapered high cut, $61-63 \mathrm{~Hz}$ tapered low cut

Bandstop: $175-179 \mathrm{~Hz}$ tapered high cut, 181-185 Hz tapered low cut

Bandpass: $10-20 \mathrm{~Hz}$ tapered low cut, $270-300 \mathrm{~Hz}$ tapered high cut

$30 \mathrm{~Hz}$ frequency-domain AGC

Custom direct S-wave mute file

Full trace amplitude balance

$\mathrm{S}$-wave picks, $12 \mathrm{~ms}$ cosine taper

$250 \mathrm{~ms}$ Window

\begin{tabular}{|l|l|}
\hline PROCESSING STEP & \\
\hline Add first break pick times into trace header
\end{tabular}

Automatic gain control

Generalized muting of seismic data

TOOL

ADDPICK

AGC

MUTE

Frequency-domain time and spatially varying filter FDFILT

Frequency-domain time and spatially varying filter FDFILT

Frequency-domain time and spatially varying filter FDFILT

Zero-phase frequency-domain spectral equalisation SPEQ

Generalized muting of $\boldsymbol{f}-\boldsymbol{k}$ spectra

Trace balance

Generalized muting of seismic data

Automatic gain control

INPUT PARAMETERS

\section{Pick file, Trace header}

$250 \mathrm{~ms}$ Window

S-wave picks, $12 \mathrm{~ms}$ cosine taper

Bandstop: $57-59 \mathrm{~Hz}$ tapered high cut, $61-63 \mathrm{~Hz}$ tapered low cut

Bandstop: $175-179 \mathrm{~Hz}$ tapered high cut, $181-185 \mathrm{~Hz}$ tapered low cut Bandpass: 10-20 Hz tapered low cut, 270-300 Hz tapered high cut

$30 \mathrm{~Hz}$ frequency-domain AGC

Custom direct S-wave mute file

BALANCE Full trace amplitude balance

MUTE S-wave picks, $12 \mathrm{~ms}$ cosine taper

$250 \mathrm{~ms}$ Window 
Appendix A.4 - Globe Claritas processing operations and parameters used to processes

\section{zero-offset S Vibroseis source VSP data from 4Q66W3.}

\begin{tabular}{|c|c|c|c|}
\hline \begin{tabular}{|l|} 
Borehole \\
Data Set \\
Component \\
\end{tabular} & $\begin{array}{l}\text { 4Q66W3 } \\
\text { Zero-offset S Vibroseis } \\
\text { Vertical }\end{array}$ & & \\
\hline PROCESSING STEP & & TOOL & INPUT PARAMETERS \\
\hline Add first break pick ti & nes into trace header & ADDPICK & Pick file, Trace header \\
\hline Spiking Wiener decon & olution & DECONW & Flattened to P-wave picks, $100 \mathrm{~ms}$ operator, $0.1 \%$ pre-whitening \\
\hline Frequency-domain tim & e and spatially varying filter & FDFILT & Bandstop: $57-59 \mathrm{~Hz}$ tapered high cut, $61-63 \mathrm{~Hz}$ tapered low cut \\
\hline Frequency-domain tim & e and spatially varying filter & FDFILT & Bandstop: $175-179 \mathrm{~Hz}$ tapered high cut, $181-185 \mathrm{~Hz}$ tapered low cut \\
\hline Frequency-domain tim & e and spatially varying filter & FDFILT & Bandpass: $15-25 \mathrm{~Hz}$ tapered low cut, $250-270 \mathrm{~Hz}$ tapered high cut \\
\hline Zero-phase frequency & domain spectral equalisation & SPEQ & $30 \mathrm{~Hz}$ frequency-domain AGC \\
\hline Generalized muting of & $f-k$ spectra & FKMUTE & Flattened to $\mathrm{P}$-wave picks, custom direct P-wave mute file \\
\hline Generalized muting of & $f-k$ spectra & FKMUTE & Custom direct $\mathrm{P}$-wave mute file \\
\hline Generalized muting 0 & $f-k$ spectra & FKMUTE & Custom aliased P-wave mute file \\
\hline Generalized muting of & $f-k$ spectra & FKMUTE & Custom direct S-wave mute file \\
\hline FX-domain complex w & einer deconvolution & FX DECON & 12-trace filter applied to 24 traces, $200 \mathrm{~ms}$ window \\
\hline Mutes to input trace $h$ & ader value & ZEROMUTE & P-wave picks, $40 \mathrm{~ms}$ cosine taper \\
\hline Automatic gain contro & & AGC & $250 \mathrm{~ms}$ Window \\
\hline Borehole & 4Q66W3 & & \\
\hline Data Set & Zero-offset & & \\
\hline Component & Transverse & & \\
\hline PROCESSING STEP & & TOOL & INPUT PARAMETERS \\
\hline Add first break pick ti & nes into trace header & ADDPICK & Pick file, Trace header \\
\hline Automatic gain contro & & AGC & $250 \mathrm{~ms}$ Window \\
\hline Generalized muting of & seismic data & MUTE & S-wave picks, $12 \mathrm{~ms}$ cosine taper \\
\hline Frequency-domain tim & and spatially varying filter & FDFILT & Bandstop: $57-59 \mathrm{~Hz}$ tapered high cut, $61-63 \mathrm{~Hz}$ tapered low cut \\
\hline Frequency-domain tim & and spatially varying filter & FDFILT & Bandstop: $175-179 \mathrm{~Hz}$ tapered high cut, $181-185 \mathrm{~Hz}$ tapered low cut \\
\hline Frequency-domain tim & and spatially varying filter & FDFILT & Bandpass: $10-20 \mathrm{~Hz}$ tapered low cut, $270-300 \mathrm{~Hz}$ tapered high cut \\
\hline Zero-phase frequency- & lomain spectral equalisation & SPEQ & $30 \mathrm{~Hz}$ frequency-domain $\mathrm{AGC}$ \\
\hline Generalized muting of & - $k$ spectra & FKMUTE & Custom direct S-wave mute file \\
\hline Trace balance & & BALANCE & Full trace amplitude balance \\
\hline Generalized muting of & ismic data & MUTE & S-wave picks, $12 \mathrm{~ms}$ cosine taper \\
\hline Automatic gain contro & & AGC & $250 \mathrm{~ms}$ Window \\
\hline Borehole & 4Q66W3 & & \\
\hline Dat & & & \\
\hline Component & Radial & & \\
\hline PROCESSING STEP & & TOOL & INPUT PARAMETERS \\
\hline Add first break pick ti & nes into trace header & ADDPICK & Pick file, Trace header \\
\hline Automatic gain contro & & AGC & $250 \mathrm{~ms}$ Window \\
\hline Generalized muting of & seismic data & MUTE & S-wave picks, $12 \mathrm{~ms}$ cosine taper \\
\hline Frequency-domain tim & and spatially varying filter & FDFILT & Bandstop: $57-59 \mathrm{~Hz}$ tapered high cut, $61-63 \mathrm{~Hz}$ tapered low cut \\
\hline Frequency-domain tim & and spatially varying filter & FDFILT & Bandstop: $175-179 \mathrm{~Hz}$ tapered high cut, $181-185 \mathrm{~Hz}$ tapered low cut \\
\hline Frequency-domain tim & and spatially varying filter & FDFILT & Bandpass: $10-20 \mathrm{~Hz}$ tapered low cut, $270-300 \mathrm{~Hz}$ tapered high cut \\
\hline Zero-phase frequency- & lomain spectral equalisation & SPEQ & $30 \mathrm{~Hz}$ frequency-domain $\mathrm{AGC}$ \\
\hline Generalized muting of & $f-k$ spectra & FKMUTE & Custom direct S-wave mute file \\
\hline Trace balance & & BALANCE & Full trace amplitude balance \\
\hline Generalized muting of & seismic data & MUTE & S-wave picks, $12 \mathrm{~ms}$ cosine taper \\
\hline Automatic gain contro & & AGC & $250 \mathrm{~ms}$ Window \\
\hline
\end{tabular}


Appendix A.5 - Globe Claritas processing operations and parameters used to processes

\section{zero-offset dynamite source VSP data from FFM001.}

\begin{tabular}{|c|c|c|c|}
\hline $\begin{array}{l}\text { Borehole } \\
\text { Data Set } \\
\text { Component } \\
\end{array}$ & $\begin{array}{l}\text { FFM001 } \\
\text { Zero-offset dynamite } \\
\text { Vertical }\end{array}$ & & \\
\hline PROCESSING STEP & & TOOL & INPUT PARAMETERS \\
\hline & ADDPICK & Pick file, Trace header \\
\hline \multicolumn{2}{|c|}{ Spiking Wiener deconvolution } & DECONW & Flattened to P-wave picks, $100 \mathrm{~ms}$ operator, $0.1 \%$ pre-whitening \\
\hline & ZEROMUTE & P-wave picks, $40 \mathrm{~ms}$ cosine taper \\
\hline \multicolumn{2}{|c|}{ Frequency-domain time and spatially varying filter } & FDFILT & Bandstop: $57-59 \mathrm{~Hz}$ tapered high cut, $61-63 \mathrm{~Hz}$ tapered low cut \\
\hline \multicolumn{2}{|c|}{ Frequency-domain time and spatially varying filter } & FDFILT & Bandstop: $97-99 \mathrm{~Hz}$ tapered high cut, $101-103 \mathrm{~Hz}$ tapered low cut \\
\hline \multicolumn{2}{|c|}{ Frequency-domain time and spatially varying filter } & FDFILT & Bandstop: $117-119 \mathrm{~Hz}$ tapered high cut, $121-123 \mathrm{~Hz}$ tapered low cut \\
\hline \multicolumn{2}{|c|}{ Frequency-domain time and spatially varying filter } & FDFILT & Bandstop: $137-139 \mathrm{~Hz}$ tapered high cut, $141-143 \mathrm{~Hz}$ tapered low cut \\
\hline \multicolumn{2}{|c|}{ Frequency-domain time and spatially varying filter } & FDFILT & Bandstop: $175-179 \mathrm{~Hz}$ tapered high cut, $181-185 \mathrm{~Hz}$ tapered low cut \\
\hline \multicolumn{2}{|c|}{ Frequency-domain time and spatially varying filter } & FDFILT & Bandstop: $217-219 \mathrm{~Hz}$ tapered high cut, $221-223 \mathrm{~Hz}$ tapered low cut \\
\hline \multicolumn{2}{|c|}{ Frequency-domain time and spatially varying filter } & FDFILT & Bandstop: $237-239 \mathrm{~Hz}$ tapered high cut, $241-243 \mathrm{~Hz}$ tapered low cut \\
\hline \multicolumn{2}{|c|}{ Frequency-domain time and spatially varying filter } & FDFILT & Bandstop: $257-259 \mathrm{~Hz}$ tapered high cut, $261-263 \mathrm{~Hz}$ tapered low cut \\
\hline \multicolumn{2}{|c|}{ Frequency-domain time and spatially varying filter } & FDFILT & Bandpass: $10-20 \mathrm{~Hz}$ tapered low cut, $270-300 \mathrm{~Hz}$ tapered high cut \\
\hline \multicolumn{2}{|c|}{ Generalized muting of $f-k$ spectra } & FKMUTE & Flattened to P-wave picks, custom direct P-wave mute file \\
\hline \multicolumn{2}{|c|}{ Generalized muting of $f-k$ spectra } & FKMUTE & Custom direct P-wave mute file \\
\hline \multicolumn{2}{|c|}{ Generalized muting of $f-k$ spectra } & FKMUTE & Custom aliased P-wave mute file \\
\hline \multicolumn{2}{|c|}{ Generalized muting of $f-k$ spectra } & FKMUTE & Custom direct S-wave mute file \\
\hline \multicolumn{2}{|l|}{ Trace balance } & BALANCE & Full trace amplitude balance \\
\hline \multicolumn{2}{|c|}{ Mutes to input trace header value } & ZEROMUTE & P-wave picks, $40 \mathrm{~ms}$ cosine taper \\
\hline \multicolumn{2}{|l|}{ Bulk static shift } & BULKSHIFT & $-50 \mathrm{~ms}$ \\
\hline \multicolumn{2}{|l|}{ Automatic gain control } & $\mathrm{AGC}$ & $250 \mathrm{~ms}$ Window \\
\hline
\end{tabular}

\begin{tabular}{|c|c|c|c|}
\hline $\begin{array}{l}\text { Borehole } \\
\text { Data Set } \\
\text { Component }\end{array}$ & $\begin{array}{l}\text { FFM001 } \\
\text { Zero-offset dynamite } \\
\text { Transverse }\end{array}$ & & \\
\hline PROCESSING STEP & & TOOL & INPUT PARAMETERS \\
\hline \multicolumn{2}{|c|}{ Add first break pick times into trace header } & ADDPICK & Pick file, Trace header \\
\hline \multicolumn{2}{|c|}{ Automatic gain control } & AGC & $250 \mathrm{~ms}$ Window \\
\hline \multicolumn{2}{|c|}{ Generalized muting of seismic data } & MUTE & S-wave picks, $12 \mathrm{~ms}$ cosine taper \\
\hline \multicolumn{2}{|c|}{ Frequency-domain time and spatially varying filter } & FDFILT & Bandstop: $57-59 \mathrm{~Hz}$ tapered high cut, $61-63 \mathrm{~Hz}$ tapered low cut \\
\hline \multicolumn{2}{|c|}{ Frequency-domain time and spatially varying filter } & FDFILT & Bandstop: $97-99 \mathrm{~Hz}$ tapered high cut, $101-103 \mathrm{~Hz}$ tapered low cut \\
\hline \multicolumn{2}{|c|}{ Frequency-domain time and spatially varying filter } & FDFILT & Bandstop: $117-119 \mathrm{~Hz}$ tapered high cut, $121-123 \mathrm{~Hz}$ tapered low cut \\
\hline \multicolumn{2}{|c|}{ Frequency-domain time and spatially varying filter } & FDFILT & Bandstop: $137-139 \mathrm{~Hz}$ tapered high cut, $141-143 \mathrm{~Hz}$ tapered low cut \\
\hline \multicolumn{2}{|c|}{ Frequency-domain time and spatially varying filter } & FDFILT & Bandstop: $175-179 \mathrm{~Hz}$ tapered high cut, $181-185 \mathrm{~Hz}$ tapered low cut \\
\hline \multicolumn{2}{|c|}{ Frequency-domain time and spatially varying filter } & FDFILT & Bandstop: $217-219 \mathrm{~Hz}$ tapered high cut, $221-223 \mathrm{~Hz}$ tapered low cut \\
\hline \multicolumn{2}{|c|}{ Frequency-domain time and spatially varying filter } & FDFILT & Bandstop: $237-239 \mathrm{~Hz}$ tapered high cut, $241-243 \mathrm{~Hz}$ tapered low cut \\
\hline \multicolumn{2}{|c|}{ Frequency-domain time and spatially varying filter } & FDFILT & Bandstop: $257-259 \mathrm{~Hz}$ tapered high cut, $261-263 \mathrm{~Hz}$ tapered low cut \\
\hline \multicolumn{2}{|c|}{ Frequency-domain time and spatially varying filter } & FDFILT & Bandpass: $10-20 \mathrm{~Hz}$ tapered low cut, $270-300 \mathrm{~Hz}$ tapered high cut \\
\hline \multirow{2}{*}{\multicolumn{2}{|c|}{ Zero-phase frequency-domain spectral equalisation }} & SPEQ & $30 \mathrm{~Hz}$ frequency-domain $\mathrm{AGC}$ \\
\hline & & FKMUTE & Custom direct S-wave mute file \\
\hline \multicolumn{2}{|c|}{ Generalized muting of seismic data } & MUTE & S-wave picks, $12 \mathrm{~ms}$ cosine taper \\
\hline \multicolumn{2}{|l|}{ Bulk static shift } & BULKSHIFT & $-50 \mathrm{~ms}$ \\
\hline \multicolumn{2}{|l|}{ Automatic gain control } & AGC & $250 \mathrm{~ms}$ Window \\
\hline
\end{tabular}




\begin{tabular}{|c|c|c|c|}
\hline $\begin{array}{l}\text { Borehole } \\
\text { Data Set } \\
\text { Component } \\
\end{array}$ & $\begin{array}{l}\text { FFM001 } \\
\text { Zero-offset dynamite } \\
\text { Radial }\end{array}$ & & \\
\hline PROCESSING STEP & & TOOL & INPUT PARAMETERS \\
\hline \multicolumn{2}{|c|}{ Add first break pick times into trace header } & ADDPICK & Pick file, Trace header \\
\hline \multicolumn{2}{|c|}{ Automatic gain control } & AGC & $250 \mathrm{~ms}$ Window \\
\hline & MUTE & S-wave picks, $12 \mathrm{~ms}$ cosine taper \\
\hline \multicolumn{2}{|c|}{ Frequency-domain time and spatially varying filter } & FDFILT & Bandstop: $57-59 \mathrm{~Hz}$ tapered high cut, $61-63 \mathrm{~Hz}$ tapered low cut \\
\hline \multicolumn{2}{|c|}{ Frequency-domain time and spatially varying filter } & FDFILT & Bandstop: $97-99 \mathrm{~Hz}$ tapered high cut, $101-103 \mathrm{~Hz}$ tapered low cut \\
\hline \multicolumn{2}{|c|}{ Frequency-domain time and spatially varying filter } & FDFILT & Bandstop: $117-119 \mathrm{~Hz}$ tapered high cut, $121-123 \mathrm{~Hz}$ tapered low cut \\
\hline \multicolumn{2}{|c|}{ Frequency-domain time and spatially varying filter } & FDFILT & Bandstop: $137-139 \mathrm{~Hz}$ tapered high cut, $141-143 \mathrm{~Hz}$ tapered low cut \\
\hline \multicolumn{2}{|c|}{ Frequency-domain time and spatially varying filter } & FDFILT & Bandstop: $175-179 \mathrm{~Hz}$ tapered high cut, $181-185 \mathrm{~Hz}$ tapered low cut \\
\hline \multicolumn{2}{|c|}{ Frequency-domain time and spatially varying filter } & FDFILT & Bandstop: $217-219 \mathrm{~Hz}$ tapered high cut, $221-223 \mathrm{~Hz}$ tapered low cut \\
\hline \multicolumn{2}{|c|}{ Frequency-domain time and spatially varying filter } & FDFILT & Bandstop: $237-239 \mathrm{~Hz}$ tapered high cut, $241-243 \mathrm{~Hz}$ tapered low cut \\
\hline \multicolumn{2}{|c|}{ Frequency-domain time and spatially varying filter } & FDFILT & Bandstop: $257-259 \mathrm{~Hz}$ tapered high cut, $261-263 \mathrm{~Hz}$ tapered low cut \\
\hline \multirow{2}{*}{\multicolumn{2}{|c|}{$\begin{array}{l}\text { Frequency-domain time and spatially varying filter } \\
\text { Zero-phase frequency-domain spectral equalisation }\end{array}$}} & FDFILT & Bandpass: $10-20 \mathrm{~Hz}$ tapered low cut, $270-300 \mathrm{~Hz}$ tapered high cut \\
\hline & & SPEQ & $30 \mathrm{~Hz}$ frequency-domain $\mathrm{AGC}$ \\
\hline \multicolumn{2}{|c|}{ Generalized muting of $f-k$ spectra } & FKMUTE & Custom direct S-wave mute file \\
\hline \multicolumn{2}{|c|}{ Generalized muting of seismic data } & MUTE & S-wave picks, $12 \mathrm{~ms}$ cosine taper \\
\hline \multicolumn{2}{|l|}{ Bulk static shift } & BULKSHIFT & $-50 \mathrm{~ms}$ \\
\hline \multicolumn{2}{|l|}{ Automatic gain control } & AGC & $250 \mathrm{~ms}$ Window \\
\hline
\end{tabular}


Appendix A.6 - Globe Claritas processing operations and parameters used to processes

\section{far-offset dynamite source VSP data from FFM001.}

\begin{tabular}{|c|c|c|c|}
\hline $\begin{array}{l}\text { Borehole } \\
\text { Data Set } \\
\text { Component }\end{array}$ & \multicolumn{3}{|l|}{$\begin{array}{l}\text { FFM001 } \\
\text { Far-offset dynamite } \\
\text { Vertical }\end{array}$} \\
\hline PROCESSING STEP & & TOOL & INPUT PARAMETERS \\
\hline \multicolumn{2}{|c|}{$\begin{array}{l}\text { Add first break pick times into trace header } \\
\text { Spiking Wiener deconvolution }\end{array}$} & ADDPICK & Pick file, Trace header \\
\hline Spiking Wiener decon & olution & DECONW & Flattened to P-wave picks, $100 \mathrm{~ms}$ operator, $0.1 \%$ pre-whitening \\
\hline \multicolumn{2}{|c|}{ Frequency-domain time and spatially varying filter } & FDFILT & Bandstop: $57-59 \mathrm{~Hz}$ tapered high cut, $61-63 \mathrm{~Hz}$ tapered low cut \\
\hline \multicolumn{2}{|c|}{ Frequency-domain time and spatially varying filter } & FDFILT & Bandstop: $97-99 \mathrm{~Hz}$ tapered high cut, $101-103 \mathrm{~Hz}$ tapered low cut \\
\hline \multicolumn{2}{|c|}{ Frequency-domain time and spatially varying filter } & FDFILT & Bandstop: $117-119 \mathrm{~Hz}$ tapered high cut, $121-123 \mathrm{~Hz}$ tapered low cut \\
\hline \multicolumn{2}{|c|}{ Frequency-domain time and spatially varying filter } & FDFILT & Bandstop: $137-139 \mathrm{~Hz}$ tapered high cut, $141-143 \mathrm{~Hz}$ tapered low cut \\
\hline \multicolumn{2}{|c|}{ Frequency-domain time and spatially varying filter } & FDFILT & Bandstop: $175-179 \mathrm{~Hz}$ tapered high cut, $181-185 \mathrm{~Hz}$ tapered low cut \\
\hline \multicolumn{2}{|c|}{ Frequency-domain time and spatially varying filter } & FDFILT & Bandstop: $217-219 \mathrm{~Hz}$ tapered high cut, $221-223 \mathrm{~Hz}$ tapered low cut \\
\hline \multicolumn{2}{|c|}{ Frequency-domain time and spatially varying filter } & FDFILT & Bandstop: $237-239 \mathrm{~Hz}$ tapered high cut, $241-243 \mathrm{~Hz}$ tapered low cut \\
\hline \multicolumn{2}{|c|}{ Frequency-domain time and spatially varying filter } & FDFILT & Bandstop: $257-259 \mathrm{~Hz}$ tapered high cut, $261-263 \mathrm{~Hz}$ tapered low cut \\
\hline \multicolumn{2}{|c|}{ Frequency-domain time and spatially varying filter } & FDFILT & Bandpass: $10-20 \mathrm{~Hz}$ tapered low cut, $270-300 \mathrm{~Hz}$ tapered high cut \\
\hline \multicolumn{2}{|c|}{ Zero-phase frequency-domain spectral equalisation } & SPEQ & $30 \mathrm{~Hz}$ frequency-domain AGC \\
\hline \multicolumn{2}{|c|}{ Generalized muting of $f-k$ spectra } & FKMUTE & Flattened to P-wave picks, custom direct P-wave mute file \\
\hline \multicolumn{2}{|c|}{ Generalized muting of $f-k$ spectra } & FKMUTE & Custom direct $\mathrm{P}$-wave mute file \\
\hline \multicolumn{2}{|c|}{ Generalized muting of $f-k$ spectra } & FKMUTE & Custom aliased P-wave mute file \\
\hline \multicolumn{2}{|c|}{ Generalized muting of $f-k$ spectra } & FKMUTE & Custom direct S-wave mute file \\
\hline \multicolumn{2}{|c|}{ Mutes to input trace header value } & ZEROMUTE & P-wave picks, $40 \mathrm{~ms}$ cosine taper \\
\hline \multicolumn{2}{|l|}{ Bulk static shift } & BULKSHIFT & $-50 \mathrm{~ms}$ \\
\hline \multicolumn{2}{|l|}{ Automatic gain control } & AGC & $250 \mathrm{~ms}$ Window \\
\hline
\end{tabular}

\begin{tabular}{|c|c|c|c|}
\hline \begin{tabular}{|l} 
Borehole \\
Data Set \\
Component
\end{tabular} & \multicolumn{3}{|l|}{$\begin{array}{l}\text { FFM001 } \\
\text { Far-offset dynamite } \\
\text { Transverse }\end{array}$} \\
\hline PROCESSING STEP & & TOOL & INPUT PARAMETERS \\
\hline \multicolumn{2}{|c|}{ Add first break pick times into trace header } & ADDPICK & Pick file, Trace header \\
\hline \multicolumn{2}{|l|}{ Automatic gain control } & AGC & $250 \mathrm{~ms}$ Window \\
\hline \multicolumn{2}{|c|}{ Mutes to input trace header value } & ZEROMUTE & P-wave picks, $40 \mathrm{~ms}$ cosine taper \\
\hline \multicolumn{2}{|c|}{ Frequency-domain time and spatially varying filter } & FDFILT & Bandstop: $57-59 \mathrm{~Hz}$ tapered high cut, $61-63 \mathrm{~Hz}$ tapered low cut \\
\hline \multicolumn{2}{|c|}{ Frequency-domain time and spatially varying filter } & FDFILT & Bandstop: $97-99 \mathrm{~Hz}$ tapered high cut, $101-103 \mathrm{~Hz}$ tapered low cut \\
\hline \multicolumn{2}{|c|}{ Frequency-domain time and spatially varying filter } & FDFILT & Bandstop: $117-119 \mathrm{~Hz}$ tapered high cut, $121-123 \mathrm{~Hz}$ tapered low cut \\
\hline \multicolumn{2}{|c|}{ Frequency-domain time and spatially varying filter } & FDFILT & Bandstop: $137-139 \mathrm{~Hz}$ tapered high cut, $141-143 \mathrm{~Hz}$ tapered low cut \\
\hline \multicolumn{2}{|c|}{ Frequency-domain time and spatially varying filter } & FDFILT & Bandstop: $175-179 \mathrm{~Hz}$ tapered high cut, $181-185 \mathrm{~Hz}$ tapered low cut \\
\hline \multicolumn{2}{|c|}{ Frequency-domain time and spatially varying filter } & FDFILT & Bandstop: $217-219 \mathrm{~Hz}$ tapered high cut, $221-223 \mathrm{~Hz}$ tapered low cut \\
\hline \multicolumn{2}{|c|}{ Frequency-domain time and spatially varying filter } & FDFILT & Bandstop: $237-239 \mathrm{~Hz}$ tapered high cut, $241-243 \mathrm{~Hz}$ tapered low cut \\
\hline \multicolumn{2}{|c|}{ Frequency-domain time and spatially varying filter } & FDFILT & Bandstop: $257-259 \mathrm{~Hz}$ tapered high cut, $261-263 \mathrm{~Hz}$ tapered low cut \\
\hline \multirow{2}{*}{\multicolumn{2}{|c|}{$\begin{array}{l}\text { Frequency-domain time and spatially varying filter } \\
\text { Zero-phase frequency-domain spectral equalisation }\end{array}$}} & FDFILT & Bandpass: $10-20 \mathrm{~Hz}$ tapered low cut, $270-300 \mathrm{~Hz}$ tapered high cut \\
\hline & & SPEQ & $30 \mathrm{~Hz}$ frequency-domain $\mathrm{AGC}$ \\
\hline \multicolumn{2}{|c|}{ Generalized muting of $f-k$ spectra } & FKMUTE & Custom direct S-wave mute file \\
\hline \multicolumn{2}{|c|}{ Mutes to input trace header value } & ZEROMUTE & P-wave picks, $40 \mathrm{~ms}$ cosine taper \\
\hline \multicolumn{2}{|l|}{ Bulk static shift } & BULKSHIFT & $-50 \mathrm{~ms}$ \\
\hline \multicolumn{2}{|l|}{ Automatic gain control } & AGC & $250 \mathrm{~ms}$ Window \\
\hline
\end{tabular}




\begin{tabular}{|c|c|c|c|}
\hline $\begin{array}{l}\text { Borehole } \\
\text { Data Set } \\
\text { Component }\end{array}$ & $\begin{array}{l}\text { FFM001 } \\
\text { Far-offset dynamite } \\
\text { Radial }\end{array}$ & & \\
\hline PROCESSING STEP & & TOOL & INPUT PARAMETERS \\
\hline \multirow{3}{*}{\multicolumn{2}{|c|}{$\begin{array}{l}\text { Add first break pick times into trace header } \\
\text { Automatic gain control } \\
\text { Mutes to input trace header value }\end{array}$}} & ADDPICK & Pick file, Trace header \\
\hline & & AGC & $250 \mathrm{~ms}$ Window \\
\hline & & ZEROMUTE & P-wave picks, $40 \mathrm{~ms}$ cosine taper \\
\hline \multicolumn{2}{|c|}{ Frequency-domain time and spatially varying filter } & FDFILT & Bandstop: $57-59 \mathrm{~Hz}$ tapered high cut, $61-63 \mathrm{~Hz}$ tapered low cut \\
\hline \multicolumn{2}{|c|}{ Frequency-domain time and spatially varying filter } & FDFILT & Bandstop: $97-99 \mathrm{~Hz}$ tapered high cut, $101-103 \mathrm{~Hz}$ tapered low cut \\
\hline \multicolumn{2}{|c|}{ Frequency-domain time and spatially varying filter } & FDFILT & Bandstop: $117-119 \mathrm{~Hz}$ tapered high cut, $121-123 \mathrm{~Hz}$ tapered low cut \\
\hline \multicolumn{2}{|c|}{ Frequency-domain time and spatially varying filter } & FDFILT & Bandstop: $137-139 \mathrm{~Hz}$ tapered high cut, $141-143 \mathrm{~Hz}$ tapered low cut \\
\hline \multicolumn{2}{|c|}{ Frequency-domain time and spatially varying filter } & FDFILT & Bandstop: $175-179 \mathrm{~Hz}$ tapered high cut, $181-185 \mathrm{~Hz}$ tapered low cut \\
\hline \multicolumn{2}{|c|}{ Frequency-domain time and spatially varying filter } & FDFILT & Bandstop: $217-219 \mathrm{~Hz}$ tapered high cut, $221-223 \mathrm{~Hz}$ tapered low cut \\
\hline \multicolumn{2}{|c|}{ Frequency-domain time and spatially varying filter } & FDFILT & Bandstop: $237-239 \mathrm{~Hz}$ tapered high cut, $241-243 \mathrm{~Hz}$ tapered low cut \\
\hline \multicolumn{2}{|c|}{ Frequency-domain time and spatially varying filter } & FDFILT & Bandstop: $257-259 \mathrm{~Hz}$ tapered high cut, $261-263 \mathrm{~Hz}$ tapered low cut \\
\hline \multirow{2}{*}{\multicolumn{2}{|c|}{$\begin{array}{l}\text { Frequency-domain time and spatially varying filter } \\
\text { Zero-phase frequency-domain spectral equalisation }\end{array}$}} & FDFILT & Bandpass: $10-20 \mathrm{~Hz}$ tapered low cut, $270-300 \mathrm{~Hz}$ tapered high cut \\
\hline & & SPEQ & $30 \mathrm{~Hz}$ frequency-domain AGC \\
\hline \multirow{2}{*}{\multicolumn{2}{|c|}{$\begin{array}{l}\text { Generalized muting of } f-k \text { spectra } \\
\text { Mutes to input trace header value }\end{array}$}} & FKMUTE & Custom direct S-wave mute file \\
\hline & & ZEROMUTE & P-wave picks, $40 \mathrm{~ms}$ cosine taper \\
\hline \multicolumn{2}{|l|}{ Bulk static shift } & BULKSHIFT & $-50 \mathrm{~ms}$ \\
\hline \multicolumn{2}{|l|}{ Automatic gain control } & AGC & $250 \mathrm{~ms}$ Window \\
\hline
\end{tabular}


Appendix A.7 - Globe Claritas processing operations and parameters used to processes

zero-offset dynamite source VSP data from FFS039.

\begin{tabular}{|c|c|c|c|}
\hline $\begin{array}{l}\text { Borehole } \\
\text { Data Set } \\
\text { Component }\end{array}$ & $\begin{array}{l}\text { FFS039 } \\
\text { Zero-offset dynamite } \\
\text { Vertical }\end{array}$ & & \\
\hline PROCESSING STEP & & TOOL & INPUT PARAMETERS \\
\hline \multirow{3}{*}{\multicolumn{2}{|c|}{$\begin{array}{l}\text { Add first break pick times into trace header } \\
\text { Spiking Wiener deconvolution } \\
\text { Mutes to input trace header value }\end{array}$}} & ADDPICK & Pick file, Trace header \\
\hline & & DECONW & Flattened to P-wave picks, $100 \mathrm{~ms}$ operator, $0.1 \%$ pre-whitening \\
\hline & & ZEROMUTE & P-wave picks, $40 \mathrm{~ms}$ cosine taper \\
\hline \multicolumn{2}{|c|}{ Frequency-domain time and spatially varying filter } & FDFILT & Bandstop: $57-59 \mathrm{~Hz}$ tapered high cut, $61-63 \mathrm{~Hz}$ tapered low cut \\
\hline \multicolumn{2}{|c|}{ Frequency-domain time and spatially varying filter } & FDFILT & Bandstop: $117-119 \mathrm{~Hz}$ tapered high cut, $121-123 \mathrm{~Hz}$ tapered low cut \\
\hline \multicolumn{2}{|c|}{ Frequency-domain time and spatially varying filter } & FDFILT & Bandstop: $175-179 \mathrm{~Hz}$ tapered high cut, $181-185 \mathrm{~Hz}$ tapered low cut \\
\hline \multicolumn{2}{|c|}{ Frequency-domain time and spatially varying filter } & FDFILT & Bandstop: $237-239 \mathrm{~Hz}$ tapered high cut, $241-243 \mathrm{~Hz}$ tapered low cut \\
\hline \multicolumn{2}{|c|}{ Frequency-domain time and spatially varying filter } & FDFILT & Bandpass: $10-20 \mathrm{~Hz}$ tapered low cut, $270-300 \mathrm{~Hz}$ tapered high cut \\
\hline \multicolumn{2}{|c|}{ Zero-phase frequency-domain spectral equalisation } & SPEQ & $30 \mathrm{~Hz}$ frequency-domain $\mathrm{AGC}$ \\
\hline \multicolumn{2}{|c|}{ Generalized muting of $f-k$ spectra } & FKMUTE & Flattened to P-wave picks, custom direct P-wave mute file \\
\hline \multicolumn{2}{|c|}{ Generalized muting of $f-k$ spectra } & FKMUTE & Custom direct P-wave mute file \\
\hline \multicolumn{2}{|c|}{ Generalized muting of $f-k$ spectra } & FKMUTE & Custom aliased $\mathrm{P}$-wave mute file \\
\hline \multicolumn{2}{|c|}{ Generalized muting of $f-k$ spectra } & FKMUTE & Custom direct S-wave mute file \\
\hline \multicolumn{2}{|l|}{ Trace balance } & BALANCE & Full trace amplitude balance \\
\hline \multicolumn{2}{|c|}{ Mutes to input trace header value } & ZEROMUTE & P-wave picks, $40 \mathrm{~ms}$ cosine taper \\
\hline \multicolumn{2}{|l|}{ Bulk static shift } & BULKSHIFT & $-50 \mathrm{~ms}$ \\
\hline \multicolumn{2}{|l|}{ Automatic gain control } & AGC & $250 \mathrm{~ms}$ Window \\
\hline
\end{tabular}

\begin{tabular}{|c|c|c|c|}
\hline $\begin{array}{l}\text { Borehole } \\
\text { Data Set } \\
\text { Component }\end{array}$ & $\begin{array}{l}\text { FFS039 } \\
\text { Zero-offset dynamite } \\
\text { Transverse }\end{array}$ & & \\
\hline PROCESSING STEP & & TOOL & INPUT PARAMETERS \\
\hline \multicolumn{2}{|c|}{$\begin{array}{l}\text { Add first break pick times into trace header } \\
\text { Automatic gain control } \\
\text { Generalized muting of seismic data } \\
\text { Frequency-domain time and spatially varying filter } \\
\text { Frequency-domain time and spatially varying filter } \\
\text { Frequency-domain time and spatially varying filter } \\
\text { Zero-phase frequency-domain spectral equalisation }\end{array}$} & $\begin{array}{l}\text { ADDPICK } \\
\text { AGC } \\
\text { MUTE } \\
\text { FDFILT } \\
\text { FDFILT } \\
\text { FDFILT } \\
\text { SPEQ } \\
\text { FKMUTE } \\
\text { MUTE } \\
\text { BULKSHIFT } \\
\text { AGC }\end{array}$ & $\begin{array}{l}\text { Pick file, Trace header } \\
250 \mathrm{~ms} \text { Window } \\
\text { S-wave picks, } 12 \mathrm{~ms} \text { cosine taper } \\
\text { Bandstop: } 57-59 \mathrm{~Hz} \text { tapered high cut, } 61-63 \mathrm{~Hz} \text { tapered low cut } \\
\text { Bandstop: } 175-179 \mathrm{~Hz} \text { tapered high cut, } 181-185 \mathrm{~Hz} \text { tapered low cut } \\
\text { Bandpass: } 10-20 \mathrm{~Hz} \text { tapered low cut, } 270-300 \mathrm{~Hz} \text { tapered high cut } \\
30 \mathrm{~Hz} \text { frequency-domain AGC } \\
\text { Custom direct S-wave mute file } \\
\text { S-wave picks, } 12 \mathrm{~ms} \text { cosine taper } \\
-50 \mathrm{~ms} \\
250 \mathrm{~ms} \text { Window }\end{array}$ \\
\hline $\begin{array}{l}\text { Borehole } \\
\text { Data Set } \\
\text { Component }\end{array}$ & $\begin{array}{l}\text { FFS039 } \\
\text { Zero-offset dynamite } \\
\text { Radial }\end{array}$ & & \\
\hline PROCESSING STEP & & TOOL & INPUT PARAMETERS \\
\hline $\begin{array}{l}\text { Add first break pick tir } \\
\text { Automatic gain control } \\
\text { Generalized muting of } \\
\text { Frequency-domain tim } \\
\text { Frequency-domain tim } \\
\text { Frequency-domain tim } \\
\text { Zero-phase frequency- } \\
\text { Generalized muting of } \\
\text { Generalized muting of } \\
\text { Bulk static shift } \\
\text { Automatic gain control }\end{array}$ & $\begin{array}{l}\text { nes into trace header } \\
\text { seismic data } \\
\text { e and spatially varying filter } \\
\text { e and spatially varying filter } \\
\text { e and spatially varying filter } \\
\text { domain spectral equalisation } \\
f-k \text { spectra } \\
\text { seismic data }\end{array}$ & $\begin{array}{l}\text { ADDPICK } \\
\text { AGC } \\
\text { MUTE } \\
\text { FDFILT } \\
\text { FDFILT } \\
\text { FDFILT } \\
\text { SPEQ } \\
\text { FKMUTE } \\
\text { MUTE } \\
\text { BULKSHIFT } \\
\text { AGC }\end{array}$ & $\begin{array}{l}\text { Pick file, Trace header } \\
250 \mathrm{~ms} \text { Window } \\
\text { S-wave picks, } 12 \mathrm{~ms} \text { cosine taper } \\
\text { Bandstop: } 57-59 \mathrm{~Hz} \text { tapered high cut, } 61-63 \mathrm{~Hz} \text { tapered low cut } \\
\text { Bandstop: } 175-179 \mathrm{~Hz} \text { tapered high cut, } 181-185 \mathrm{~Hz} \text { tapered low cut } \\
\text { Bandpass: } 10-20 \mathrm{~Hz} \text { tapered low cut, } 270-300 \mathrm{~Hz} \text { tapered high cut } \\
30 \mathrm{~Hz} \text { frequency-domain AGC } \\
\text { Custom direct S-wave mute file } \\
\text { S-wave picks, } 12 \mathrm{~ms} \text { cosine taper } \\
-50 \mathrm{~ms} \\
250 \mathrm{~ms} \text { Window }\end{array}$ \\
\hline
\end{tabular}


Appendix A.8 - Globe Claritas processing operations and parameters used to processes

\section{far-offset dynamite source VSP data from FFS039.}

\begin{tabular}{|c|c|c|c|}
\hline \begin{tabular}{|l|} 
Borehole \\
Data Set \\
Component \\
\end{tabular} & \begin{tabular}{|l|} 
FFS039 \\
Far-offset dynamite \\
Vertical
\end{tabular} & & \\
\hline PROCESSING STEP & & TOOL & INPUT PARAMETERS \\
\hline \multicolumn{2}{|c|}{ Add first break pick times into trace header } & ADDPICK & Pick file, Trace header \\
\hline \multirow{6}{*}{\multicolumn{2}{|c|}{$\begin{array}{l}\text { Spiking Wiener deconvolution } \\
\text { Frequency-domain time and spatially varying filter } \\
\text { Frequency-domain time and spatially varying filter } \\
\text { Frequency-domain time and spatially varying filter } \\
\text { Generalized muting of } f-k \text { spectra } \\
\text { Generalized muting of } f-k \text { spectra }\end{array}$}} & DECONW & Flattened to $\mathrm{P}$-wave picks, $100 \mathrm{~ms}$ operator, $0.1 \%$ pre-whitening \\
\hline & & FDFILT & Bandstop: $57-59 \mathrm{~Hz}$ tapered high cut, $61-63 \mathrm{~Hz}$ tapered low cut \\
\hline & & FDFILT & Bandstop: $175-179 \mathrm{~Hz}$ tapered high cut, $181-185 \mathrm{~Hz}$ tapered low cut \\
\hline & & FDFILT & Bandpass: $10-20 \mathrm{~Hz}$ tapered low cut, $270-300 \mathrm{~Hz}$ tapered high cut \\
\hline & & FKMUTE & Flattened to P-wave picks, custom direct P-wave mute file \\
\hline & & FKMUTE & Custom direct P-wave mute file \\
\hline \multirow{2}{*}{\multicolumn{2}{|c|}{ Generalized muting of $f-k$ spectra }} & FKMUTE & Custom aliased P-wave mute file \\
\hline Generalized muting of $f-k$ spectra & & FKMUTE & Custom direct S-wave mute file \\
\hline \multicolumn{2}{|c|}{ Mutes to input trace header value } & ZEROMUTE & P-wave picks, $40 \mathrm{~ms}$ cosine taper \\
\hline \multicolumn{2}{|c|}{ Bulk static shift } & BULKSHIFT & $-50 \mathrm{~ms}$ \\
\hline \multicolumn{2}{|l|}{ Automatic gain control } & AGC & $250 \mathrm{~ms}$ Window \\
\hline \begin{tabular}{|l|} 
Borehole \\
Data Set \\
Component
\end{tabular} & \begin{tabular}{|l|} 
FFS039 \\
Far-offset dynamite \\
Transverse
\end{tabular} & & \\
\hline PROCESSING STEP & & TOOL & INPUT PARAMETERS \\
\hline \multirow{3}{*}{\multicolumn{2}{|c|}{$\begin{array}{l}\text { Add first break pick times into trace header } \\
\text { Automatic gain control } \\
\text { Generalized muting of seismic data }\end{array}$}} & ADDPICK & Pick file, Trace header \\
\hline & & AGC & $250 \mathrm{~ms}$ Window \\
\hline & & MUTE & S-wave picks, $12 \mathrm{~ms}$ cosine taper \\
\hline \multicolumn{2}{|c|}{ Frequency-domain time and spatially varying filter } & FDFILT & Bandstop: $57-59 \mathrm{~Hz}$ tapered high cut, $61-63 \mathrm{~Hz}$ tapered low cut \\
\hline \multicolumn{2}{|c|}{ Frequency-domain time and spatially varying filter } & FDFILT & Bandstop: $175-179 \mathrm{~Hz}$ tapered high cut, $181-185 \mathrm{~Hz}$ tapered low cut \\
\hline \multirow{2}{*}{\multicolumn{2}{|c|}{$\begin{array}{l}\text { Frequency-domain time and spatially varying filter } \\
\text { Zero-phase frequency-domain spectral equalisation }\end{array}$}} & FDFILT & Bandpass: $10-20 \mathrm{~Hz}$ tapered low cut, $270-300 \mathrm{~Hz}$ tapered high cut \\
\hline & & SPEQ & $30 \mathrm{~Hz}$ frequency-domain $\mathrm{AGC}$ \\
\hline \multicolumn{2}{|c|}{$\begin{array}{l}\text { Generalized muting of } f-k \text { spectra } \\
\text { Generalized muting of seismic data }\end{array}$} & FKMUTE & Custom direct S-wave mute file \\
\hline \multicolumn{2}{|c|}{ Generalized muting of seismic data } & MUTE & S-wave picks, $12 \mathrm{~ms}$ cosine taper \\
\hline \multirow{2}{*}{\multicolumn{2}{|c|}{$\begin{array}{l}\text { Bulk static shift } \\
\text { Automatic gain control }\end{array}$}} & BULKSHIFT & $-50 \mathrm{~ms}$ \\
\hline & & AGC & $250 \mathrm{~ms}$ Window \\
\hline \multirow{2}{*}{\begin{tabular}{|l|} 
Borehole \\
Data Set \\
Component \\
\end{tabular}} & \multicolumn{3}{|l|}{ FFS039 } \\
\hline & $\begin{array}{l}\text { Far-offset dy } \\
\text { Radial }\end{array}$ & & \\
\hline PROCESSING STEP & & TOOL & INPUT PARAMETERS \\
\hline \multicolumn{2}{|c|}{\begin{tabular}{|l|} 
Add first break pick times into trace header \\
\end{tabular}} & ADDPICK & Pick file, Trace header \\
\hline \multicolumn{2}{|c|}{ Automatic gain control } & AGC & $250 \mathrm{~ms}$ Window \\
\hline \multicolumn{2}{|c|}{ Generalized muting of seismic data } & MUTE & S-wave picks, $12 \mathrm{~ms}$ cosine taper \\
\hline \multicolumn{2}{|c|}{ Frequency-domain time and spatially varying filter } & FDFILT & Bandstop: $57-59 \mathrm{~Hz}$ tapered high cut, $61-63 \mathrm{~Hz}$ tapered low cut \\
\hline \multicolumn{2}{|c|}{ Frequency-domain time and spatially varying filter } & FDFILT & Bandstop: $175-179 \mathrm{~Hz}$ tapered high cut, $181-185 \mathrm{~Hz}$ tapered low cut \\
\hline \multirow{2}{*}{\multicolumn{2}{|c|}{$\begin{array}{l}\text { Frequency-domain time and spatially varying filter } \\
\text { Zero-phase frequency-domain spectral equalisation }\end{array}$}} & FDFILT & Bandpass: $10-20 \mathrm{~Hz}$ tapered low cut, $270-300 \mathrm{~Hz}$ tapered high cut \\
\hline & & SPEQ & $30 \mathrm{~Hz}$ frequency-domain $\mathrm{AGC}$ \\
\hline \multirow{2}{*}{\multicolumn{2}{|c|}{$\begin{array}{l}\text { Generalized muting of } f-k \text { spectra } \\
\text { Generalized muting of seismic data }\end{array}$}} & FKMUTE & Custom direct S-wave mute file \\
\hline & & MUTE & S-wave picks, $12 \mathrm{~ms}$ cosine taper \\
\hline \multicolumn{2}{|l|}{ Bulk static shift } & BULKSHIFT & $-50 \mathrm{~ms}$ \\
\hline \multicolumn{2}{|l|}{ Automatic gain control } & AGC & $250 \mathrm{~ms}$ Window \\
\hline
\end{tabular}




\section{Appendix B - 3-component shot gathers for all field VSP data collected}

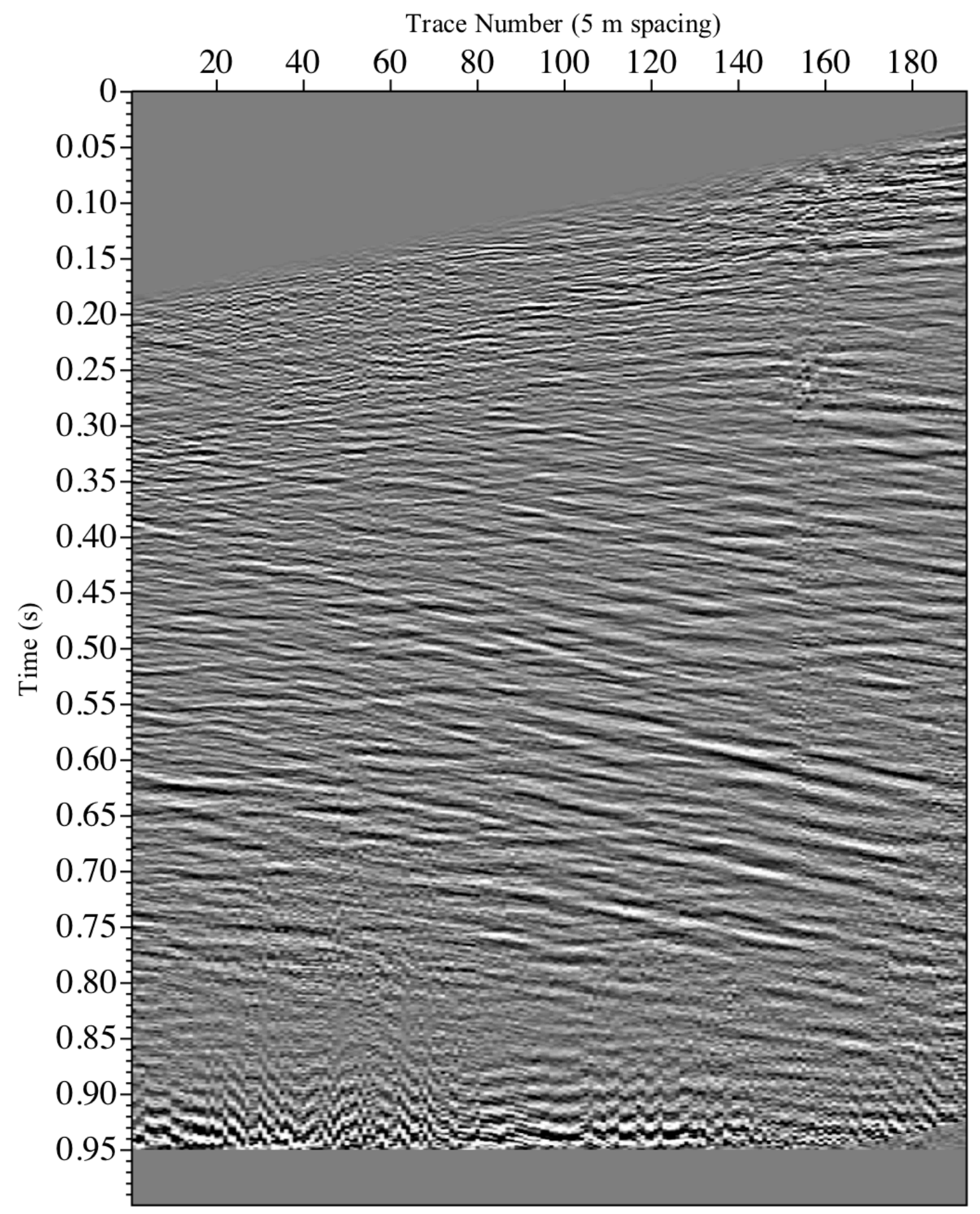

Appendix B.1 - Processed zero-offset dynamite source vertical component VSP data from 4Q66W3. 


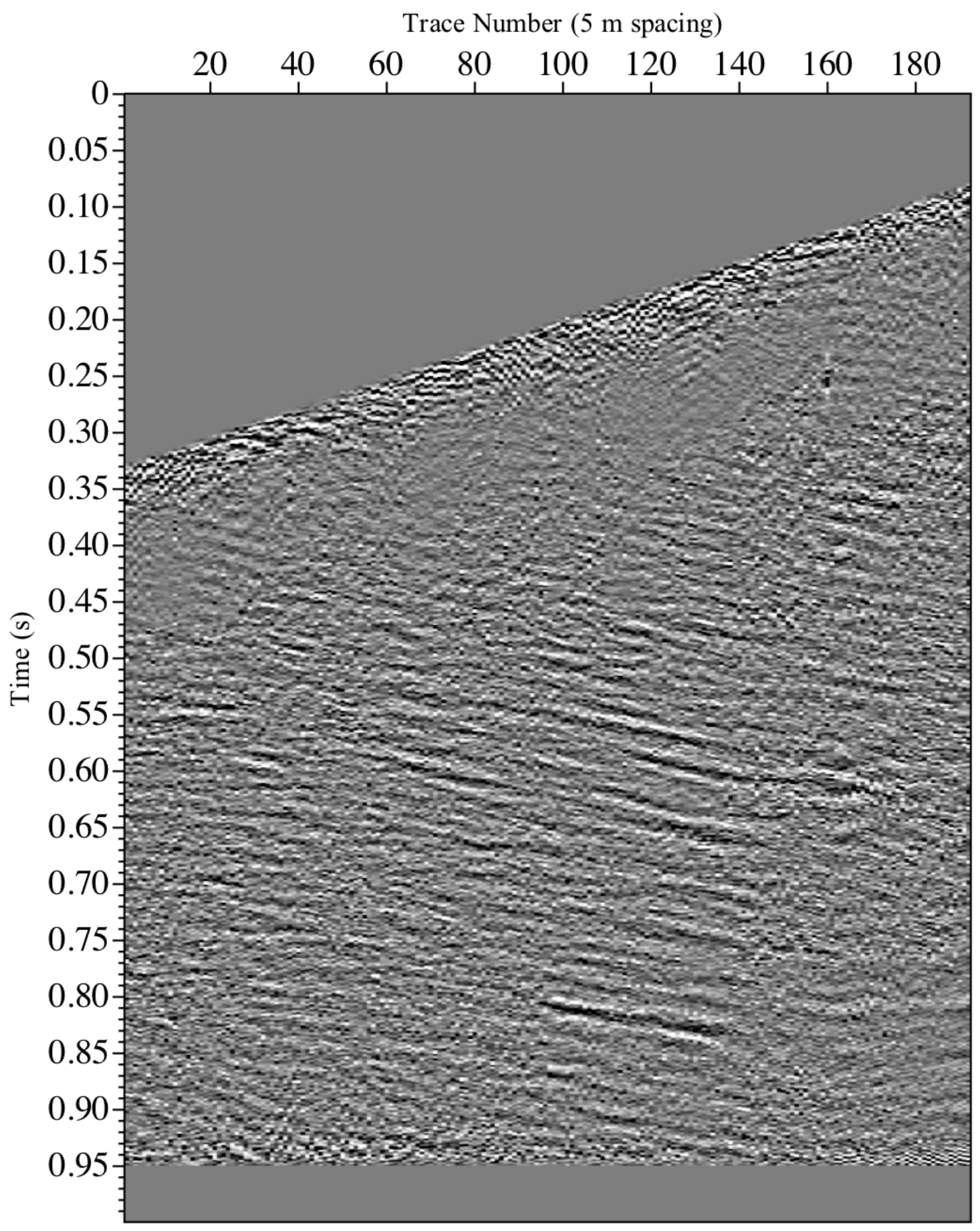

Appendix B.2 - Processed zero-offset dynamite source transverse component VSP data from 4Q66W3. 


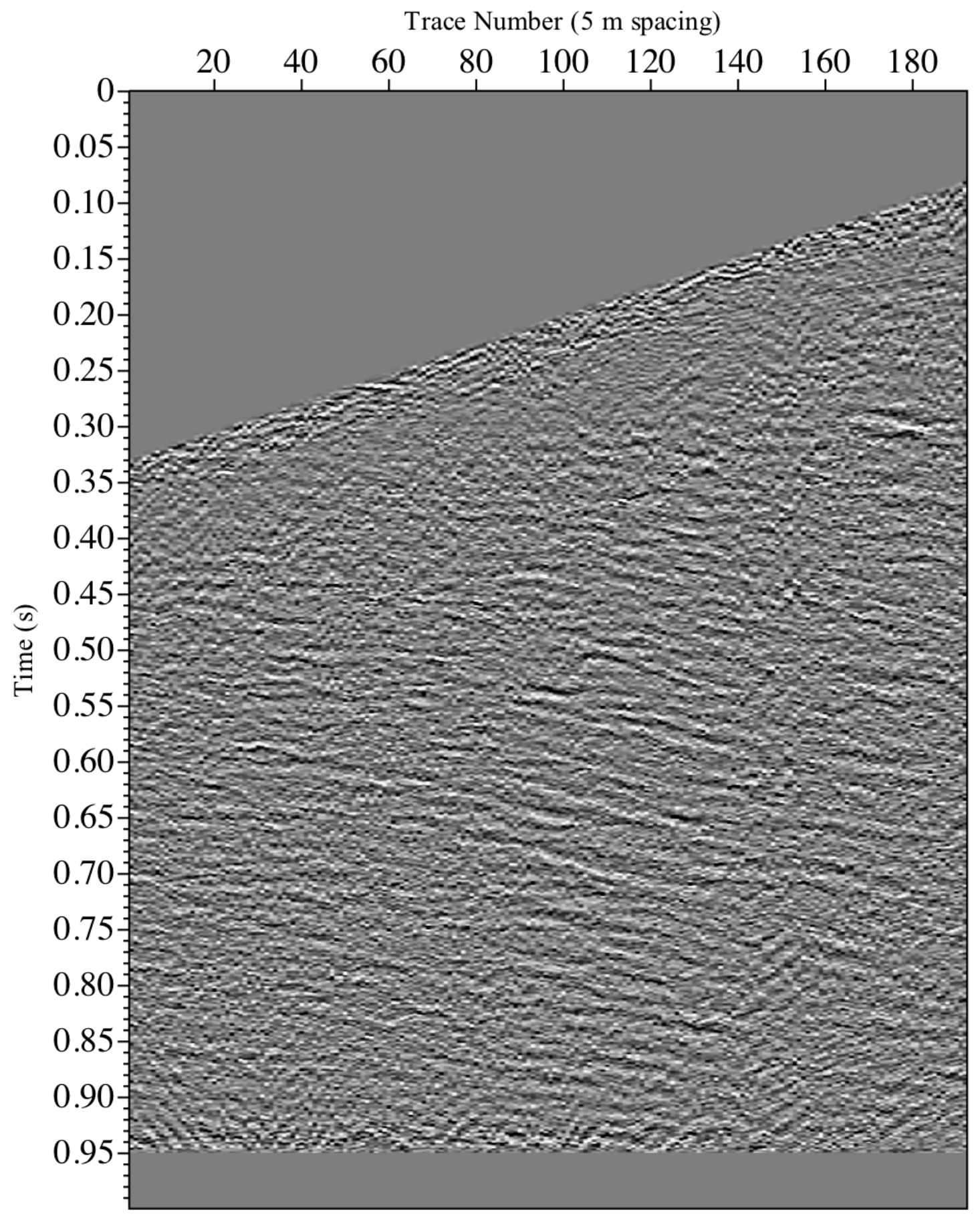

Appendix B.3 - Processed zero-offset dynamite source radial component VSP data from 4Q66W3. 


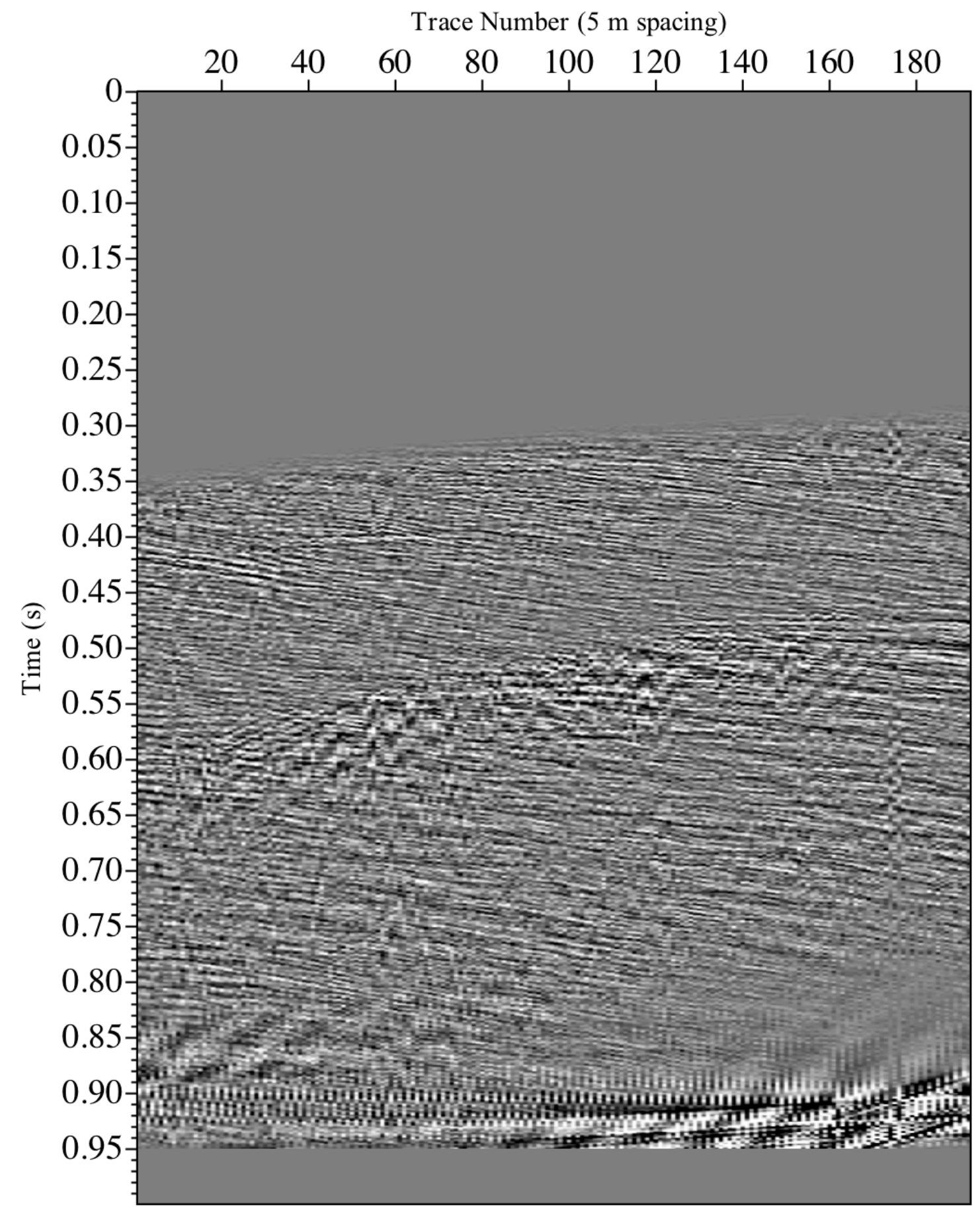

Appendix B.4 - Processed far-offset dynamite source vertical component VSP data from 4Q66W3. 


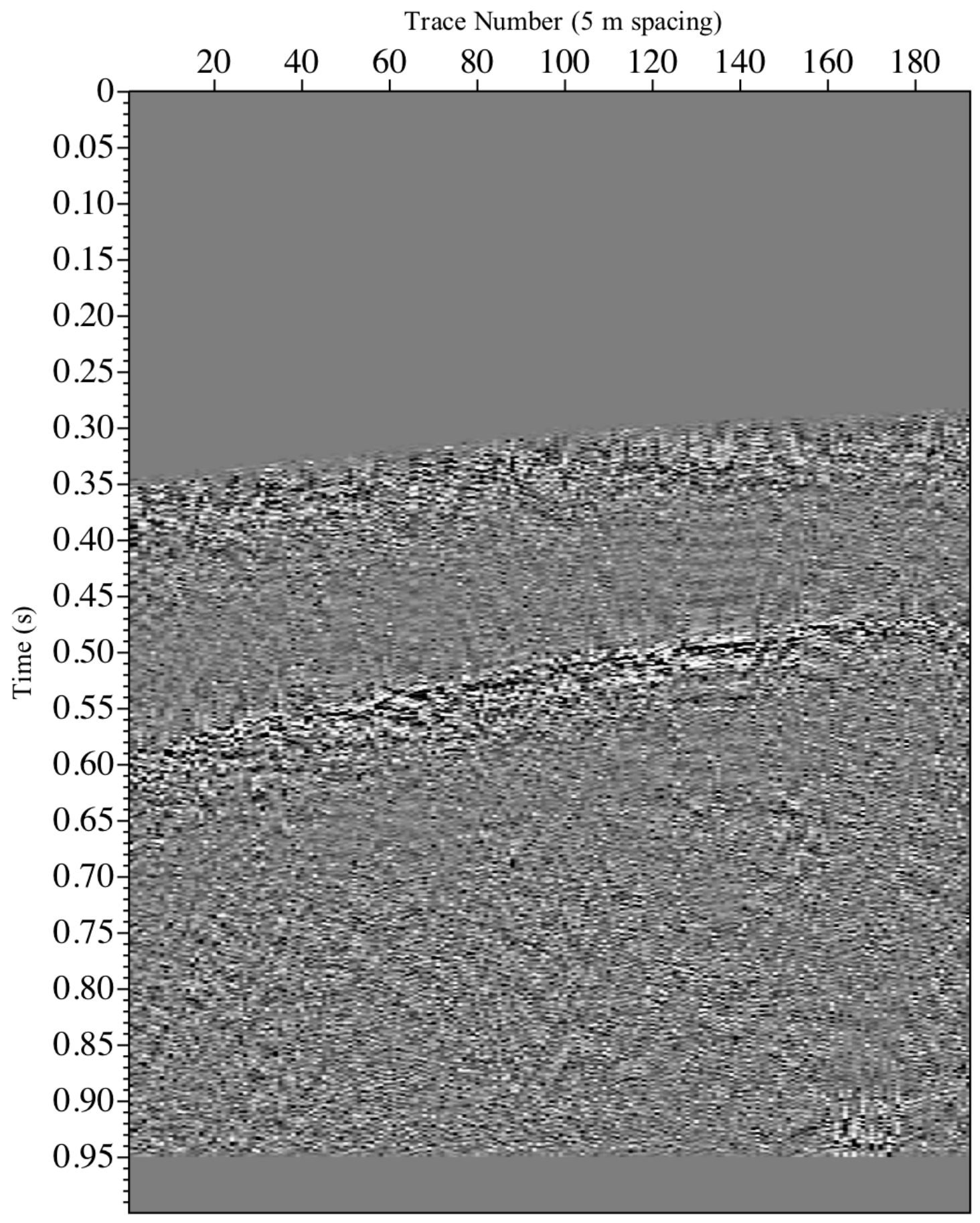

Appendix B.5 - Processed far-offset dynamite source transverse component VSP data from 4Q66W3. 


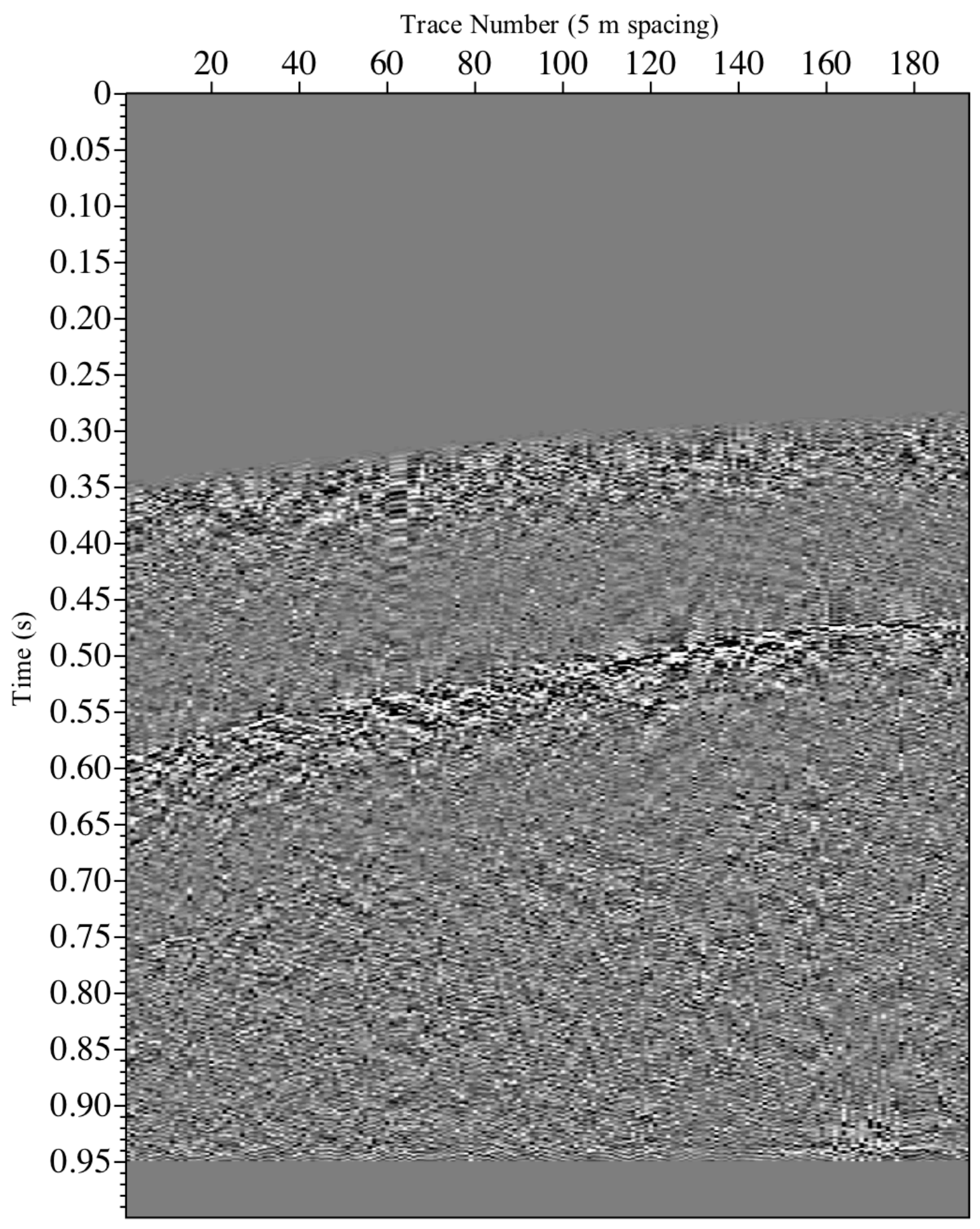

Appendix B.6 - Processed far-offset dynamite source radial component VSP data from 4Q66W3. 


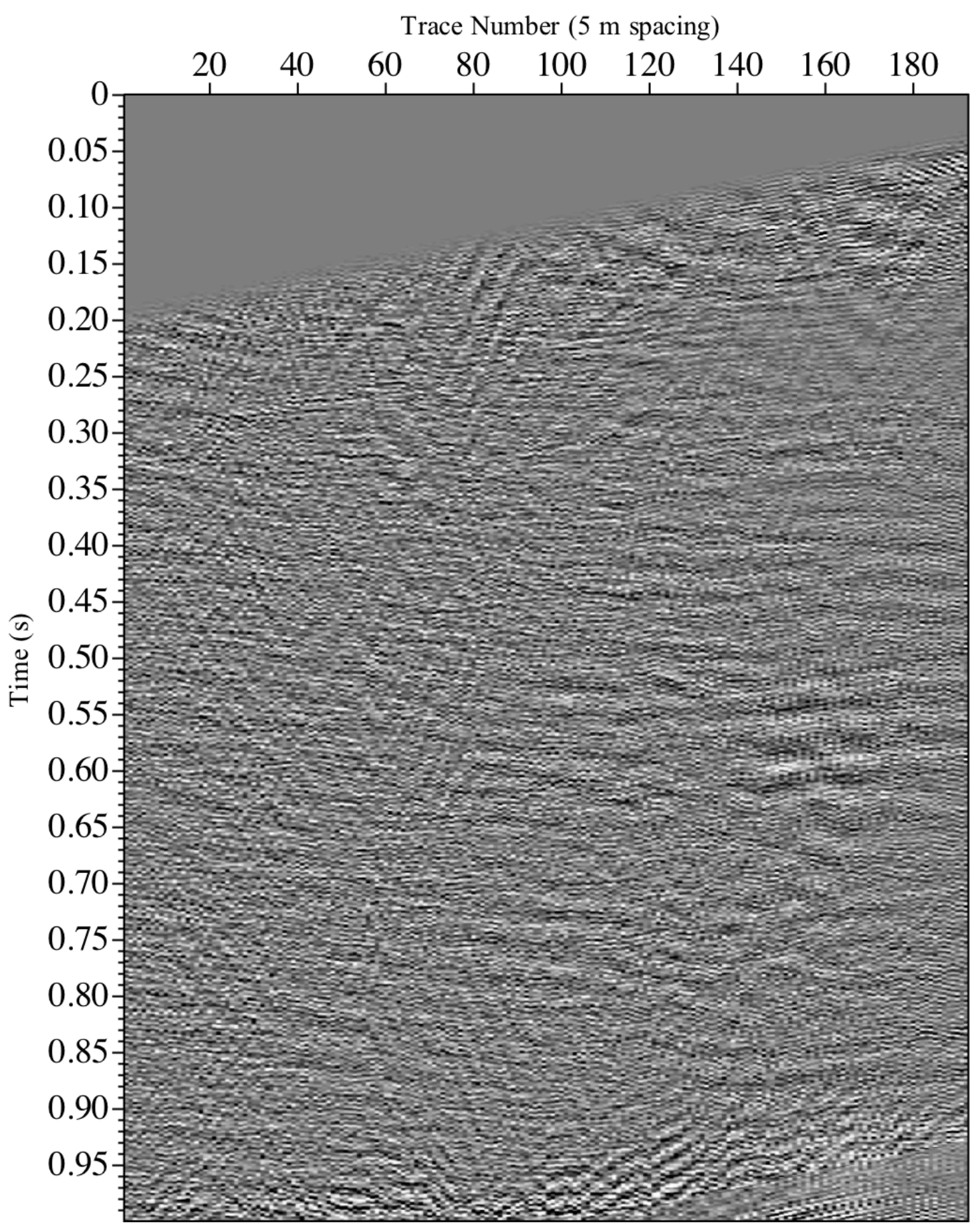

Appendix B.7 - Processed zero-offset P Vibroseis source vertical component VSP data

from 4Q66W3. 


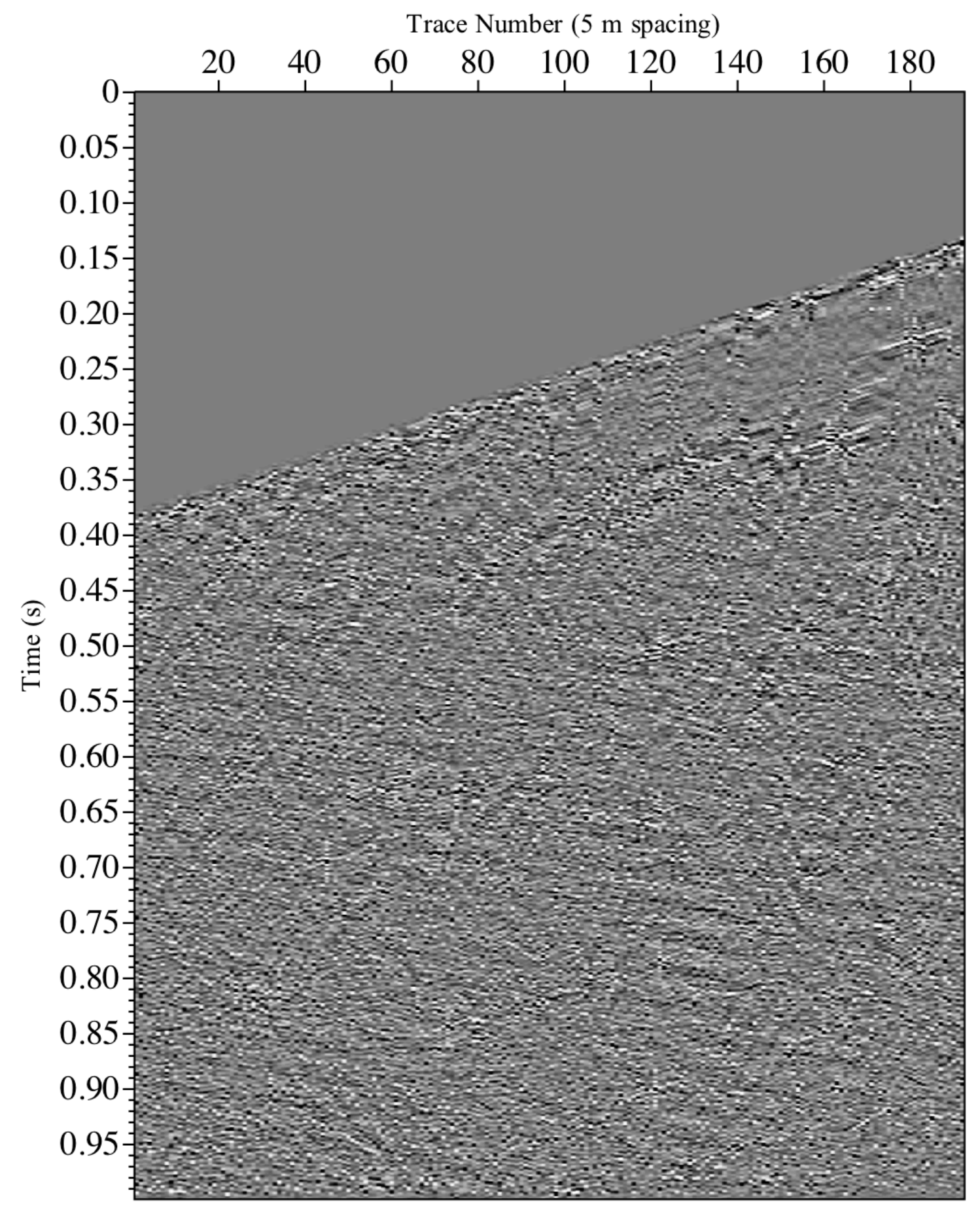

Appendix B.8 - Processed zero-offset P Vibroseis source transverse component VSP data from 4Q66W3. 


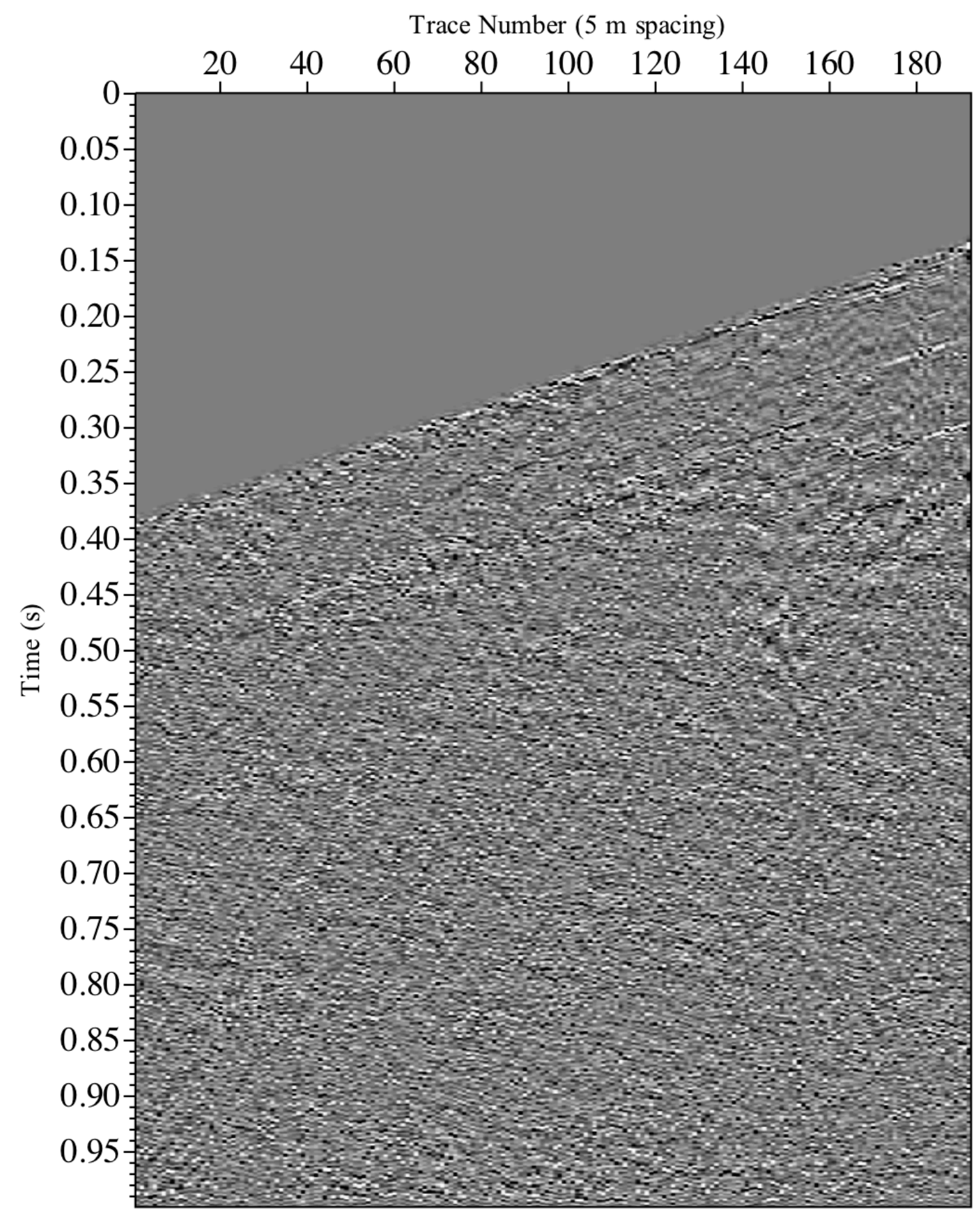

Appendix B.9 - Processed zero-offset P Vibroseis source radial component VSP data from 4Q66W3. 


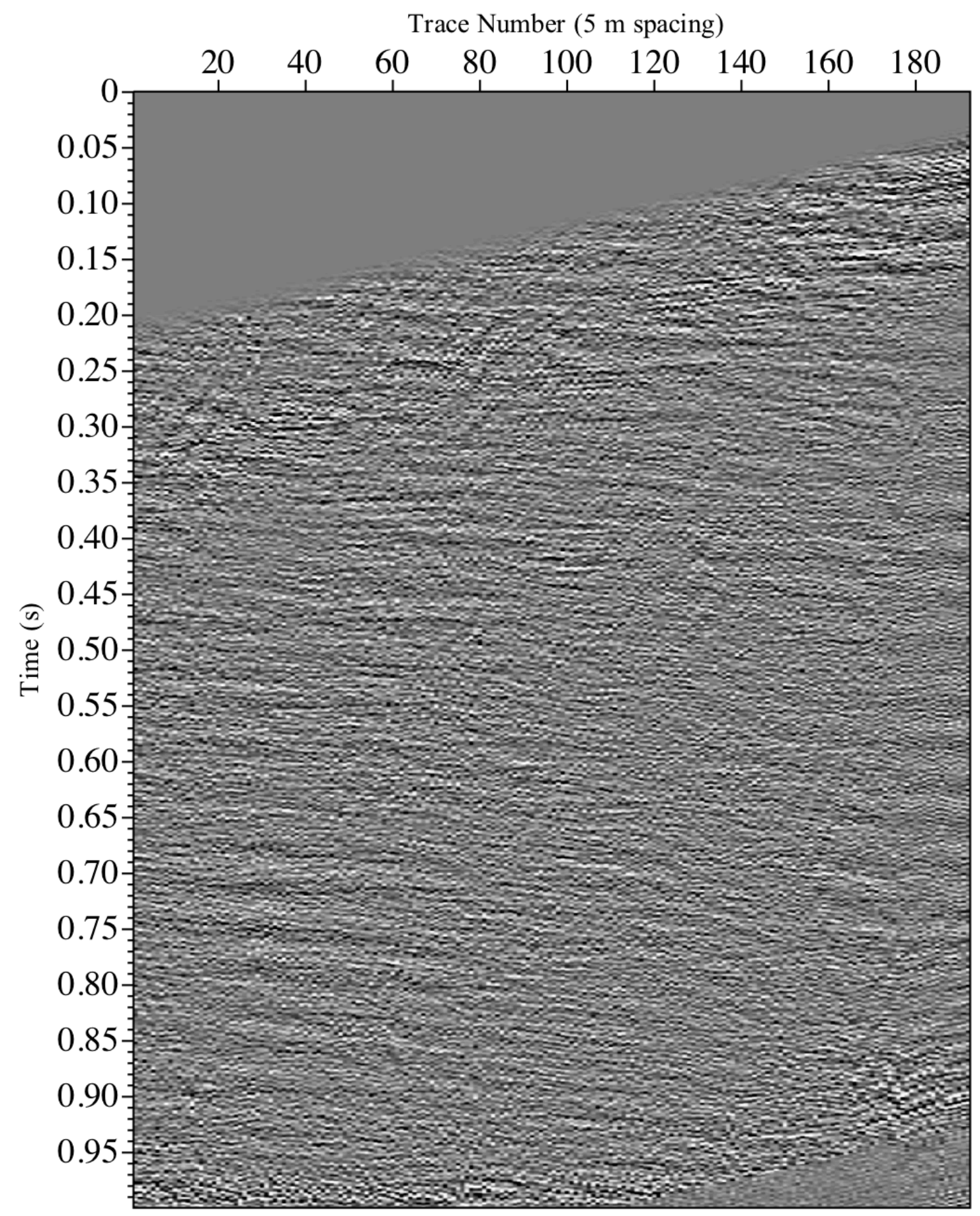

Appendix B.10 - Processed zero-offset S Vibroseis source vertical component VSP data from 4Q66W3. 


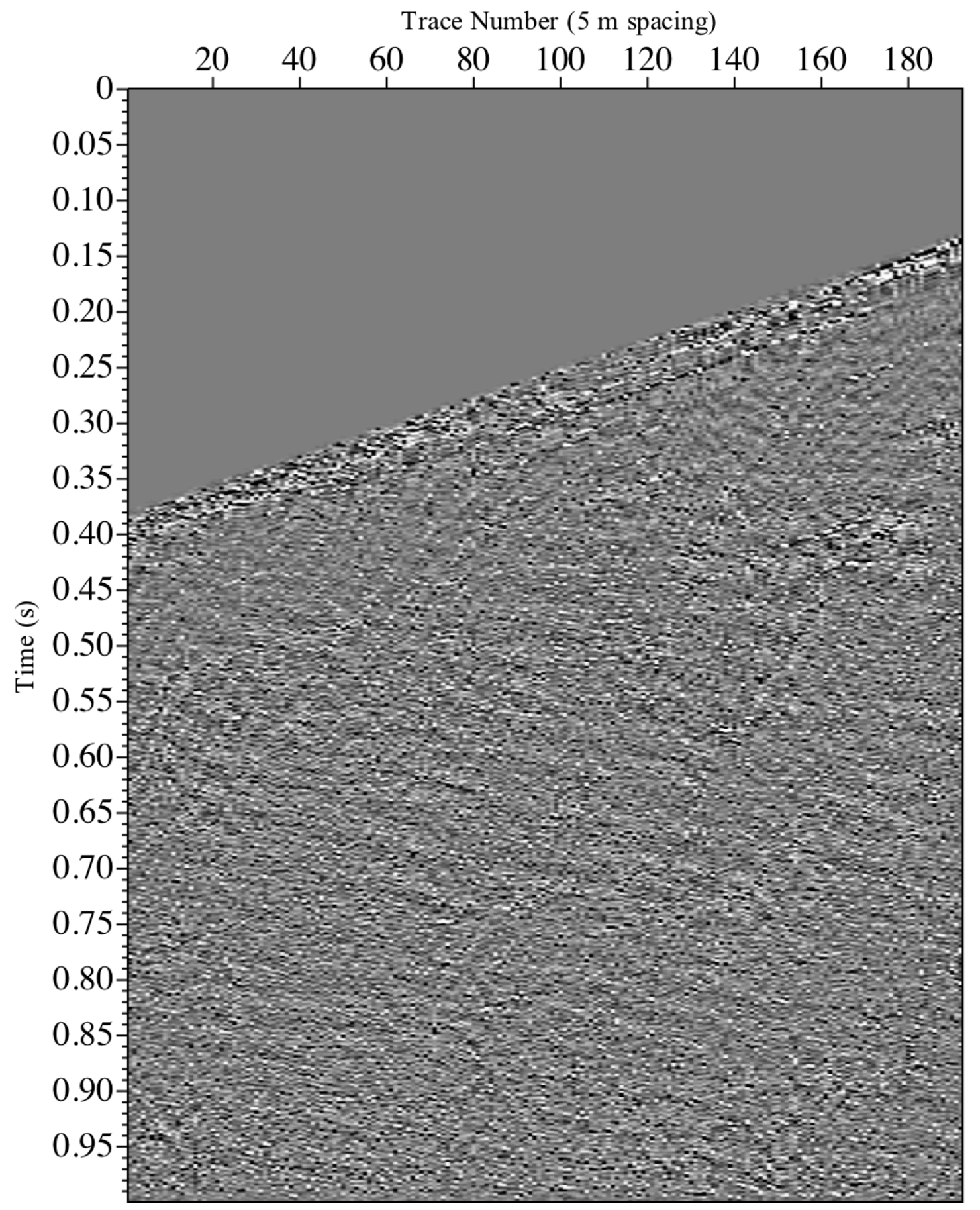

Appendix B.11 - Processed zero-offset S Vibroseis source transverse component VSP data from 4Q66W3. 


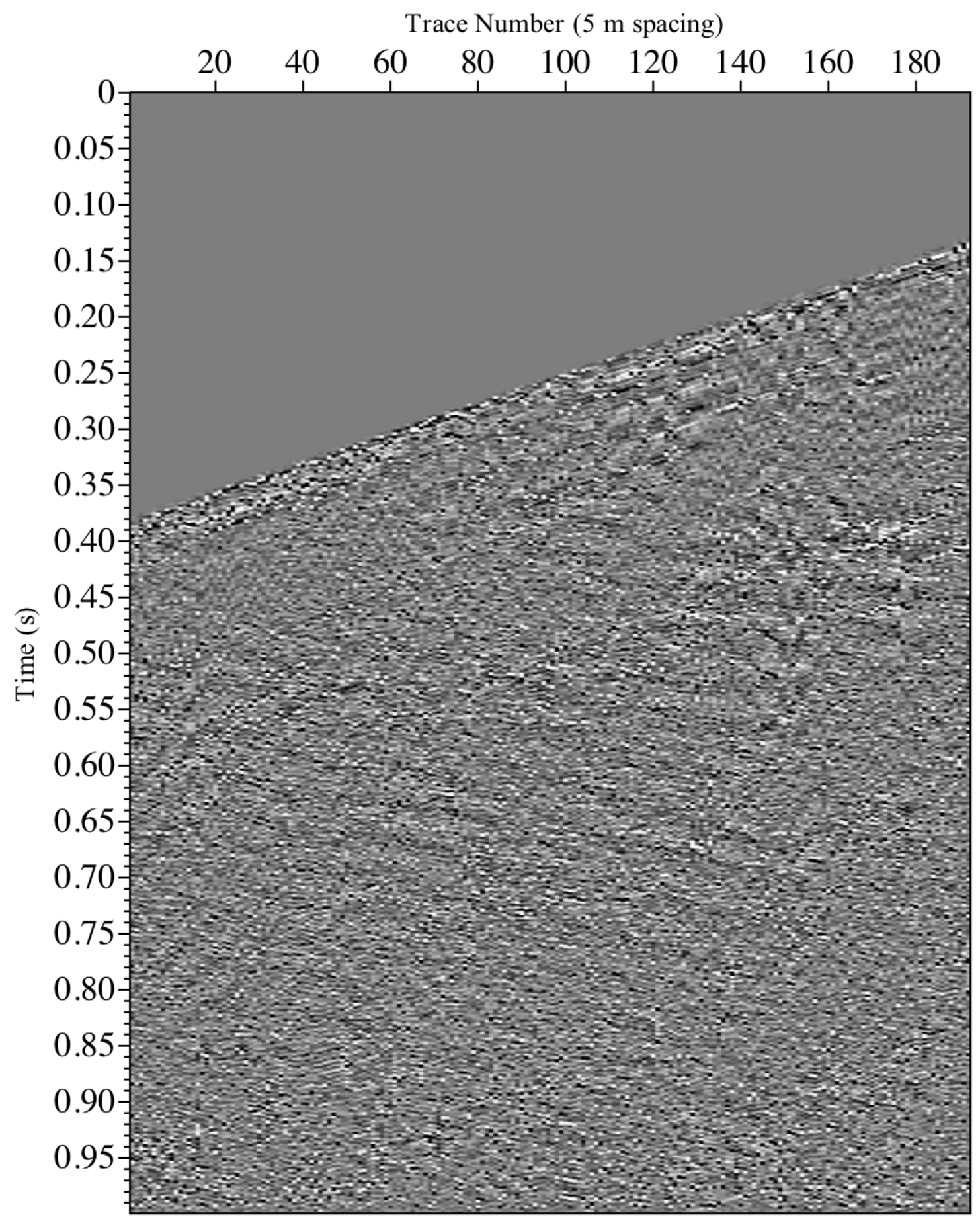

Appendix B.12 - Processed zero-offset S Vibroseis source radial component VSP data from 4Q66W3. 


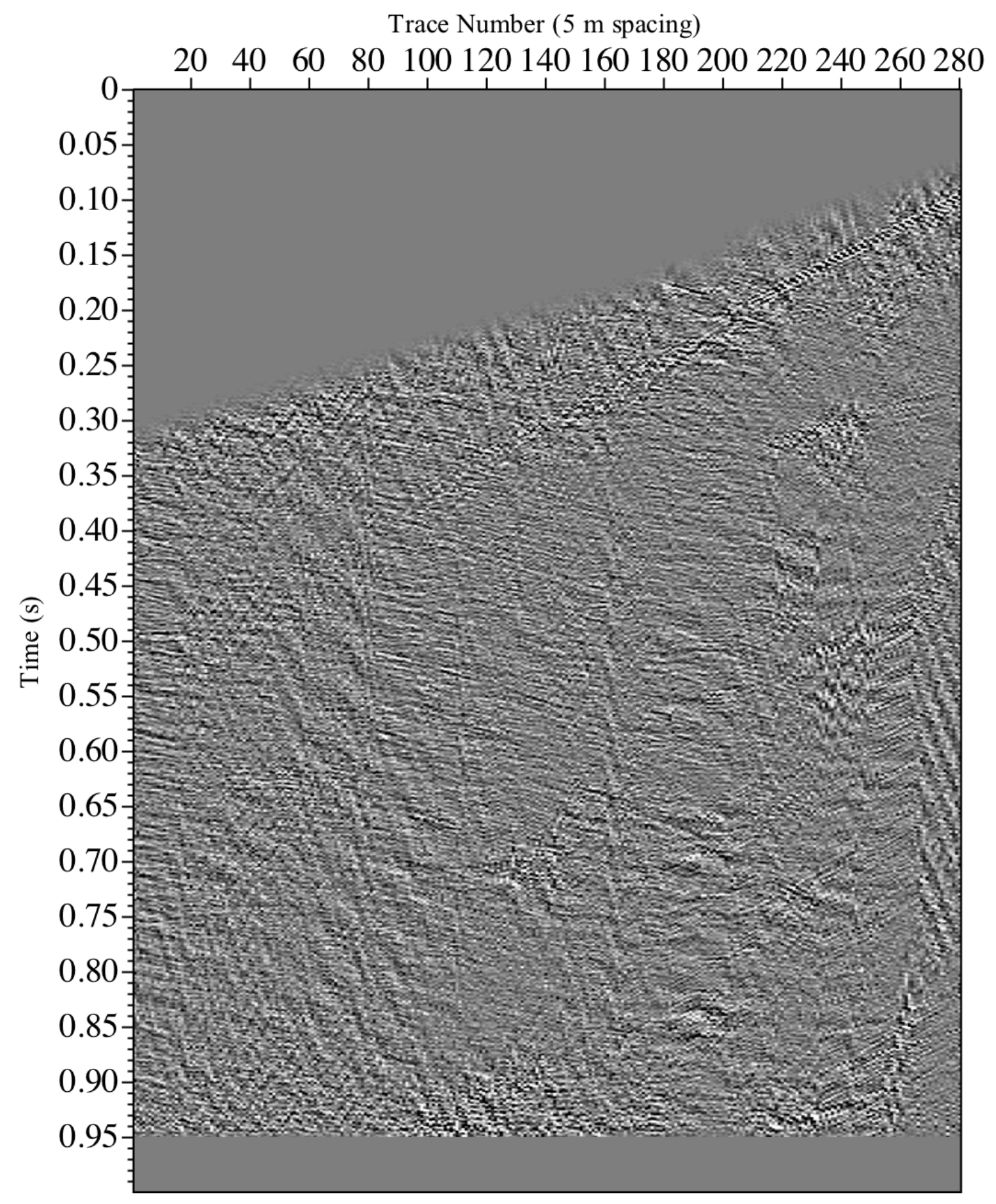

Appendix B.13 - Processed zero-offset dynamite source vertical component VSP data from FFM001. 


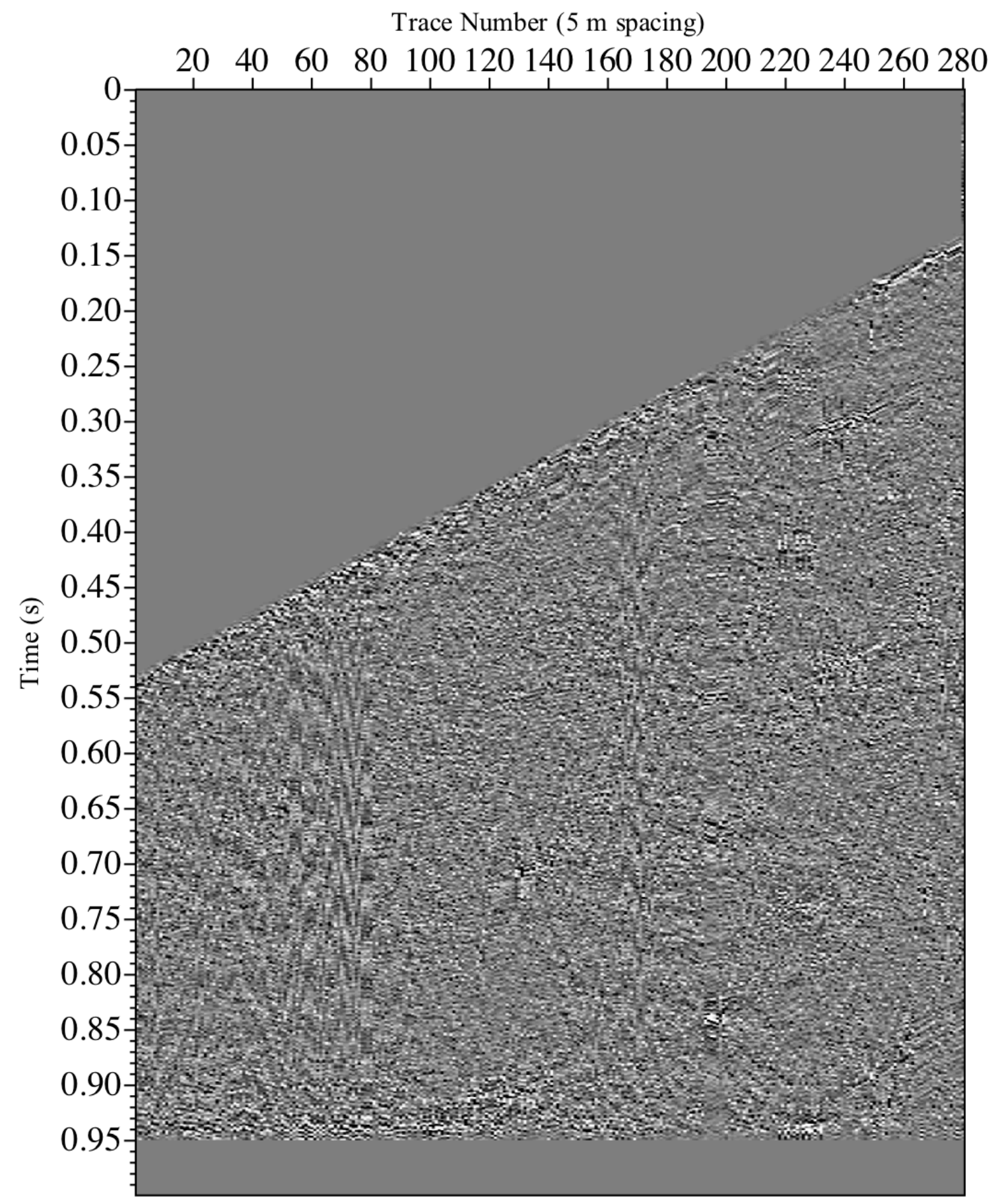

Appendix B.14 - Processed zero-offset dynamite source transverse component VSP data from FFM001. 
Trace Number (5 m spacing)

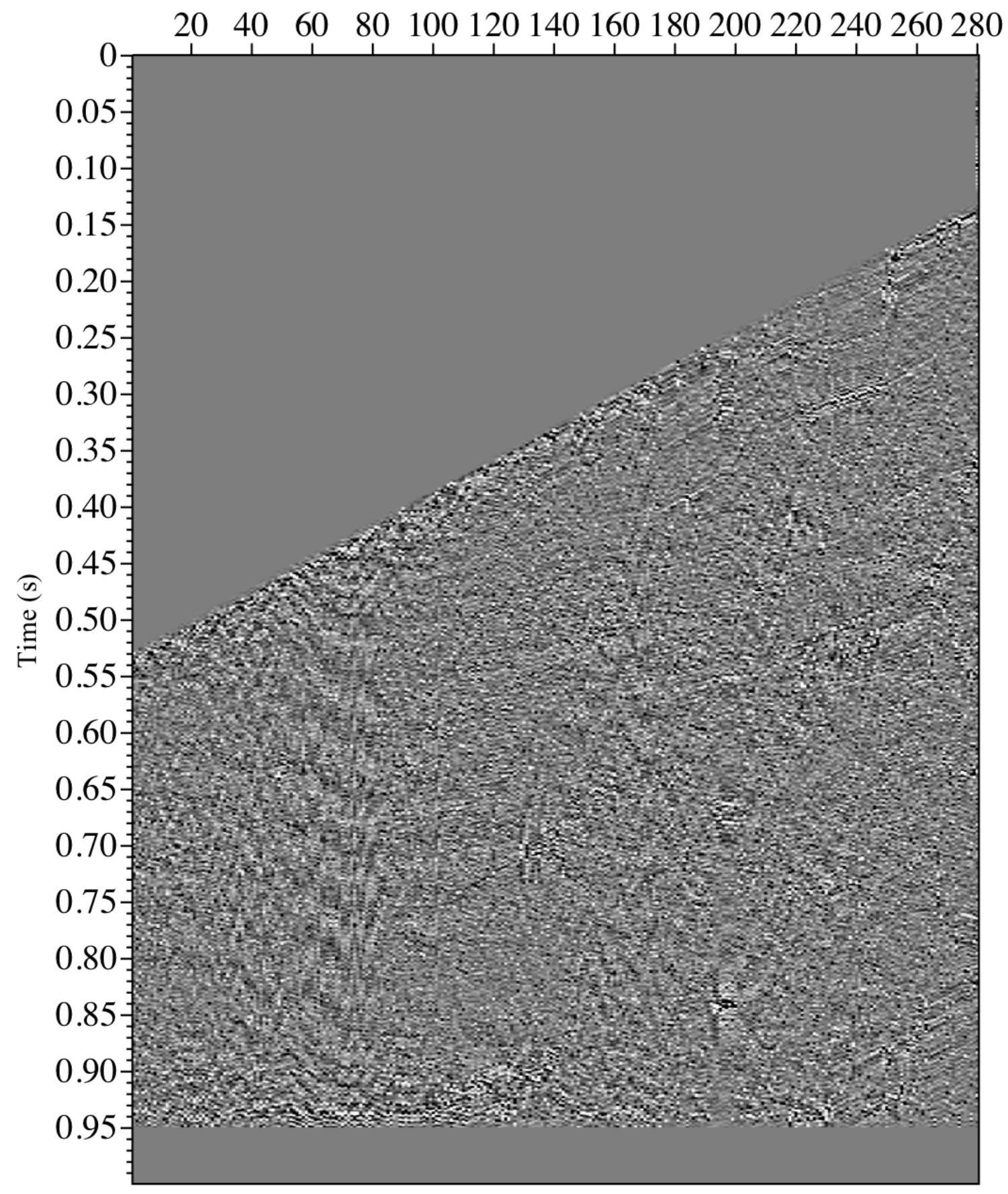

Appendix B.15 - Processed zero-offset dynamite source radial component VSP data from FFM001. 


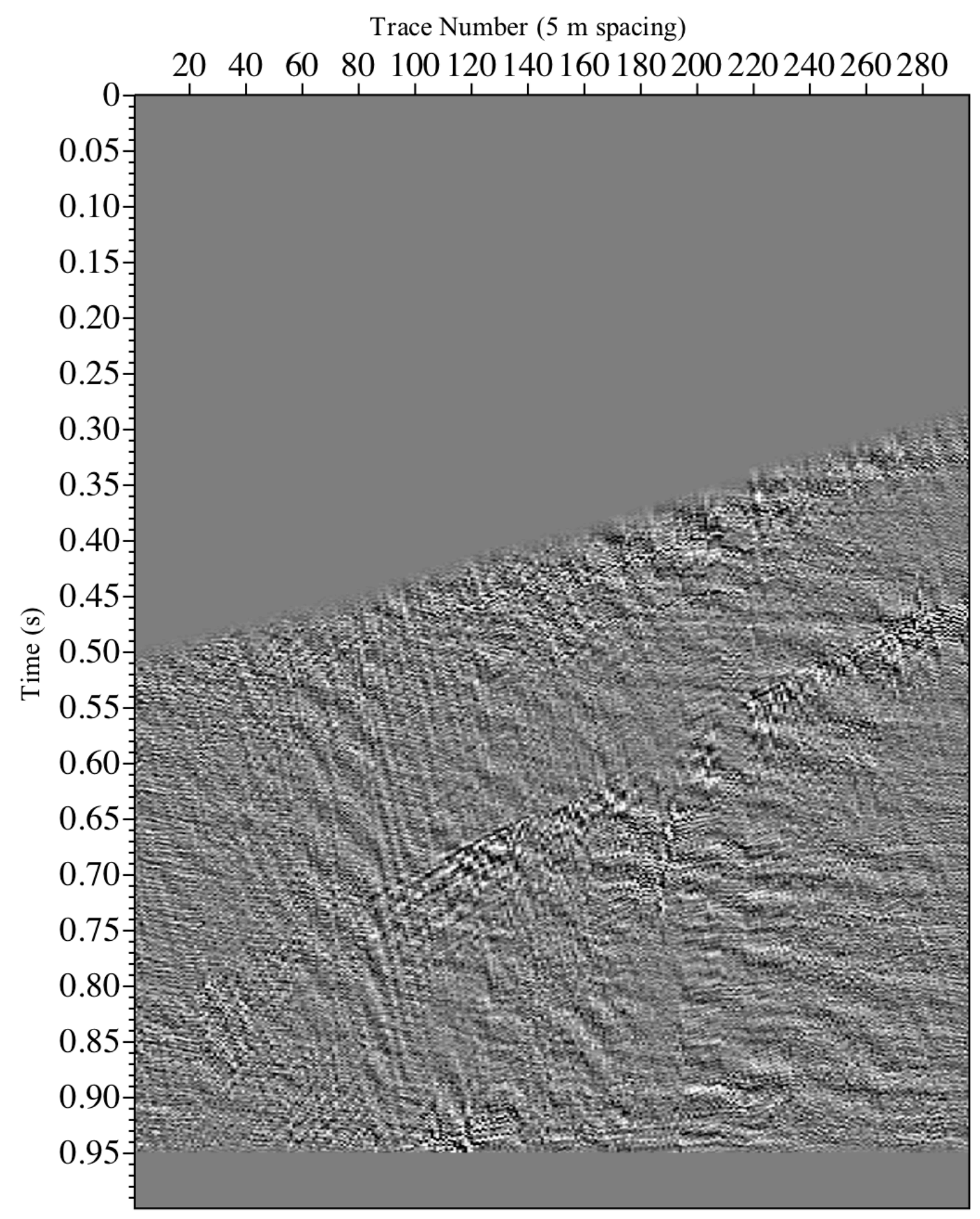

Appendix B.16 - Processed far-offset dynamite source vertical component VSP data from FFM001. 


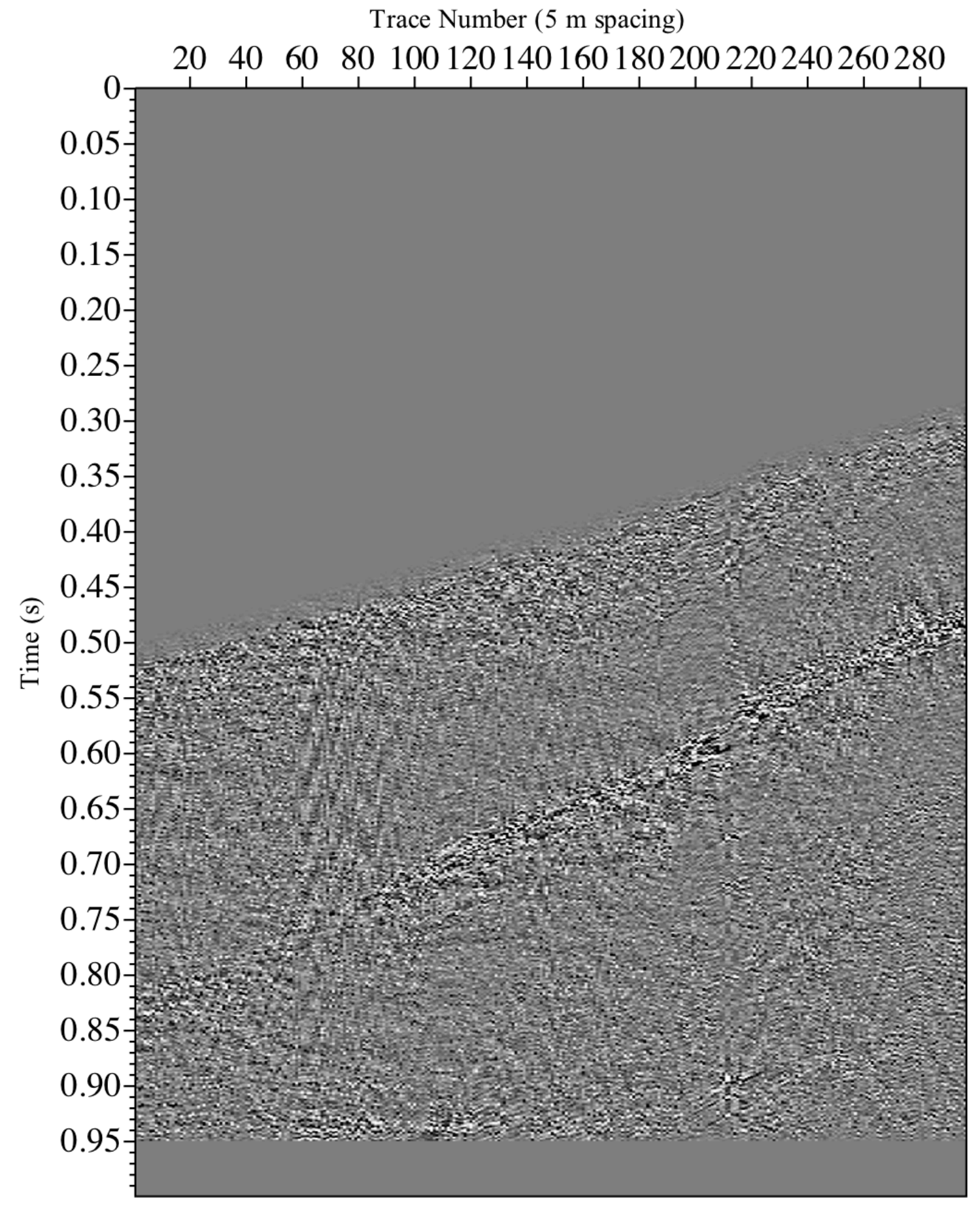

Appendix B.17 - Processed far-offset dynamite source transverse component VSP data from FFM001. 


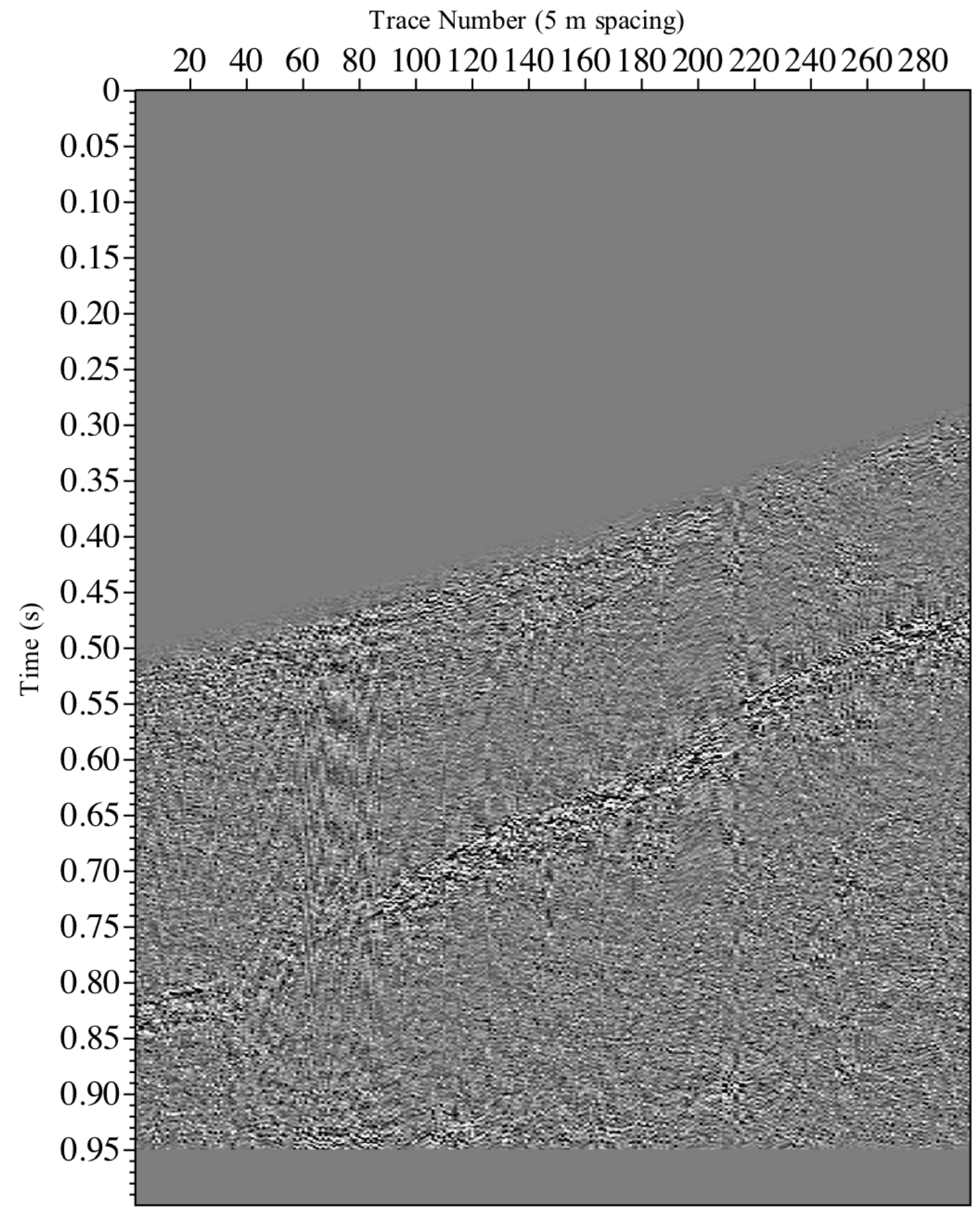

Appendix B.18 - Processed far-offset dynamite source radial component VSP data from

FFM001. 


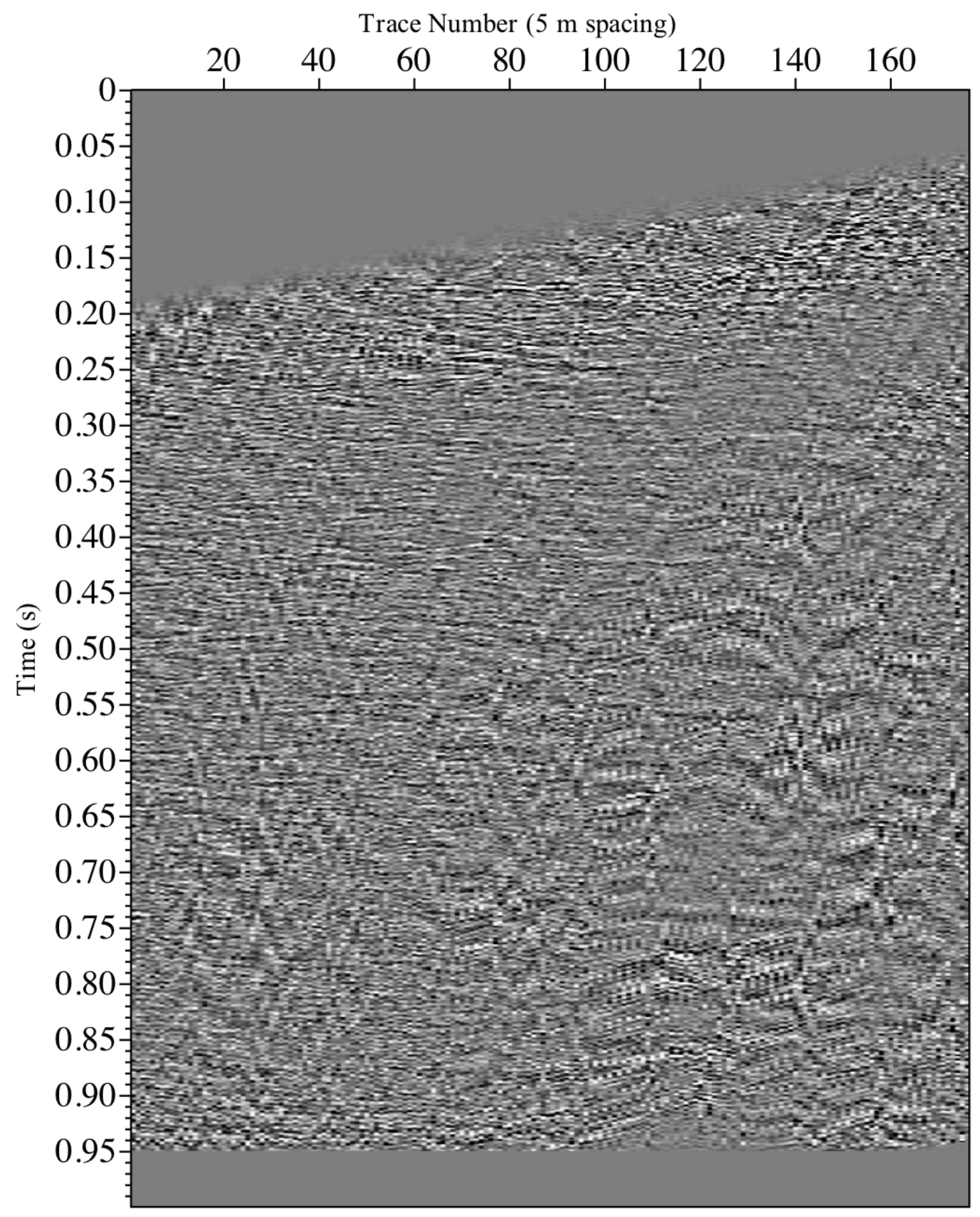

Appendix B.19 - Processed zero-offset dynamite source vertical component VSP data from FFS039. 


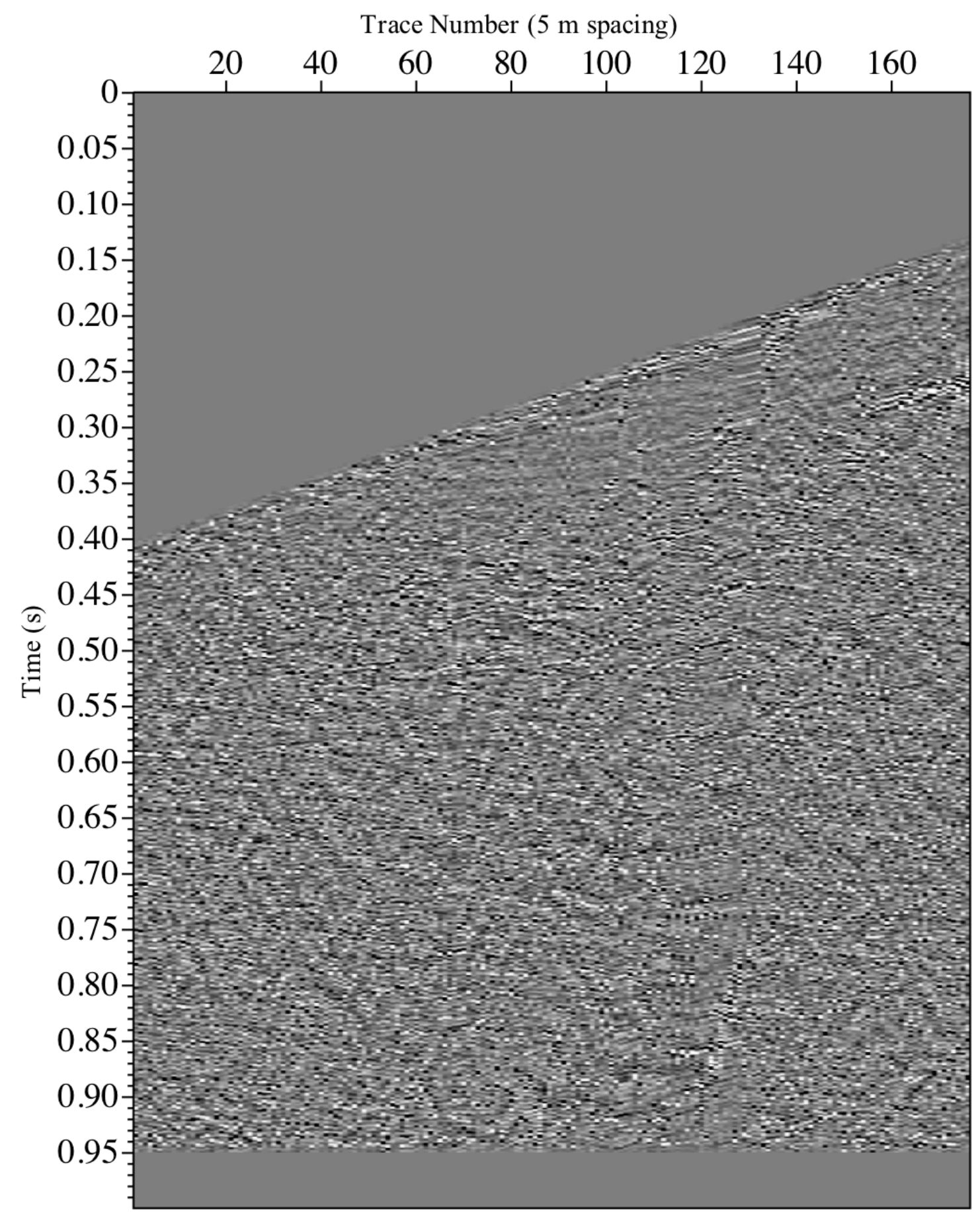

Appendix B.20 - Processed zero-offset dynamite source transverse component VSP data from FFS039. 


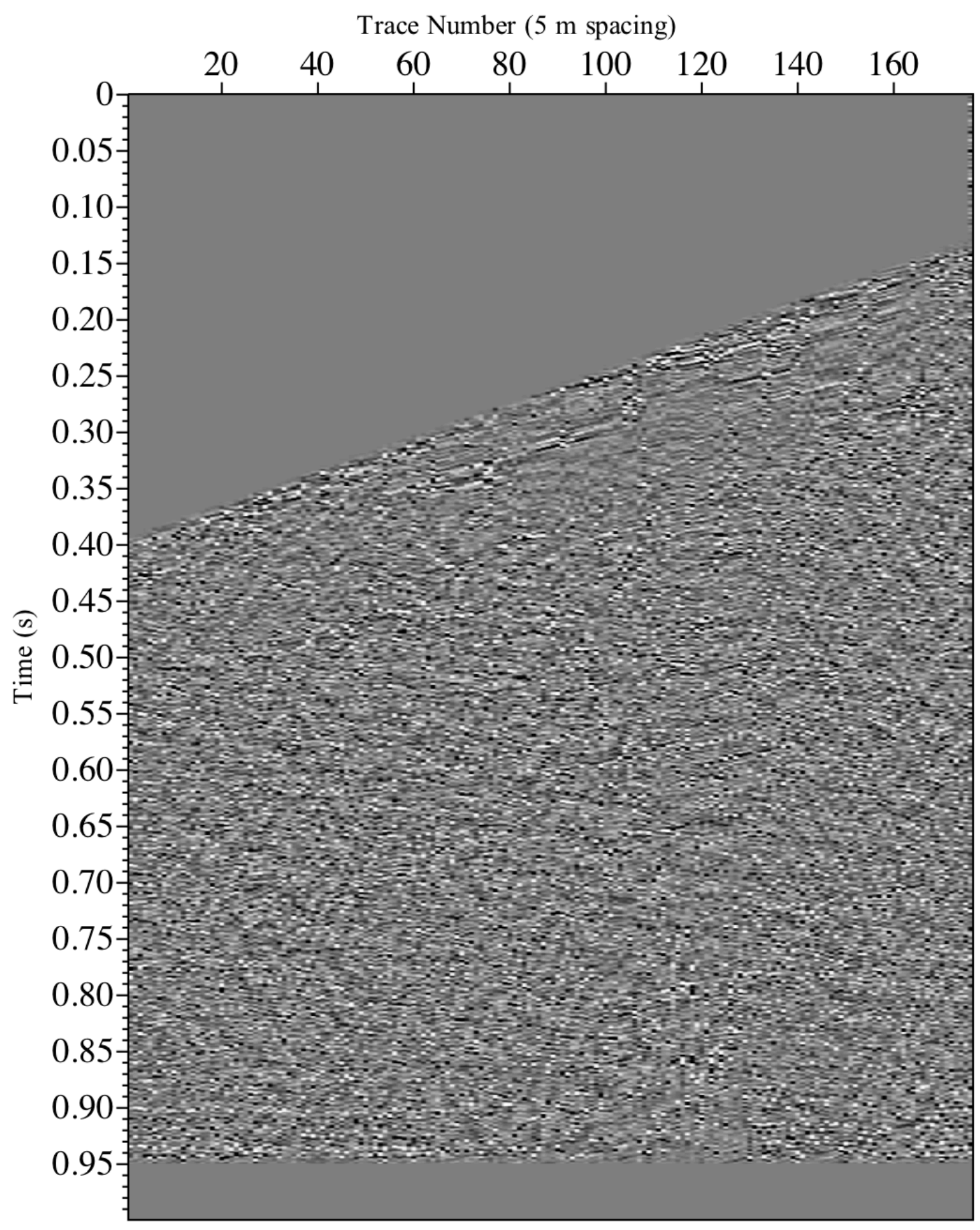

Appendix B.21 - Processed zero-offset dynamite source radial component VSP data from FFS039. 


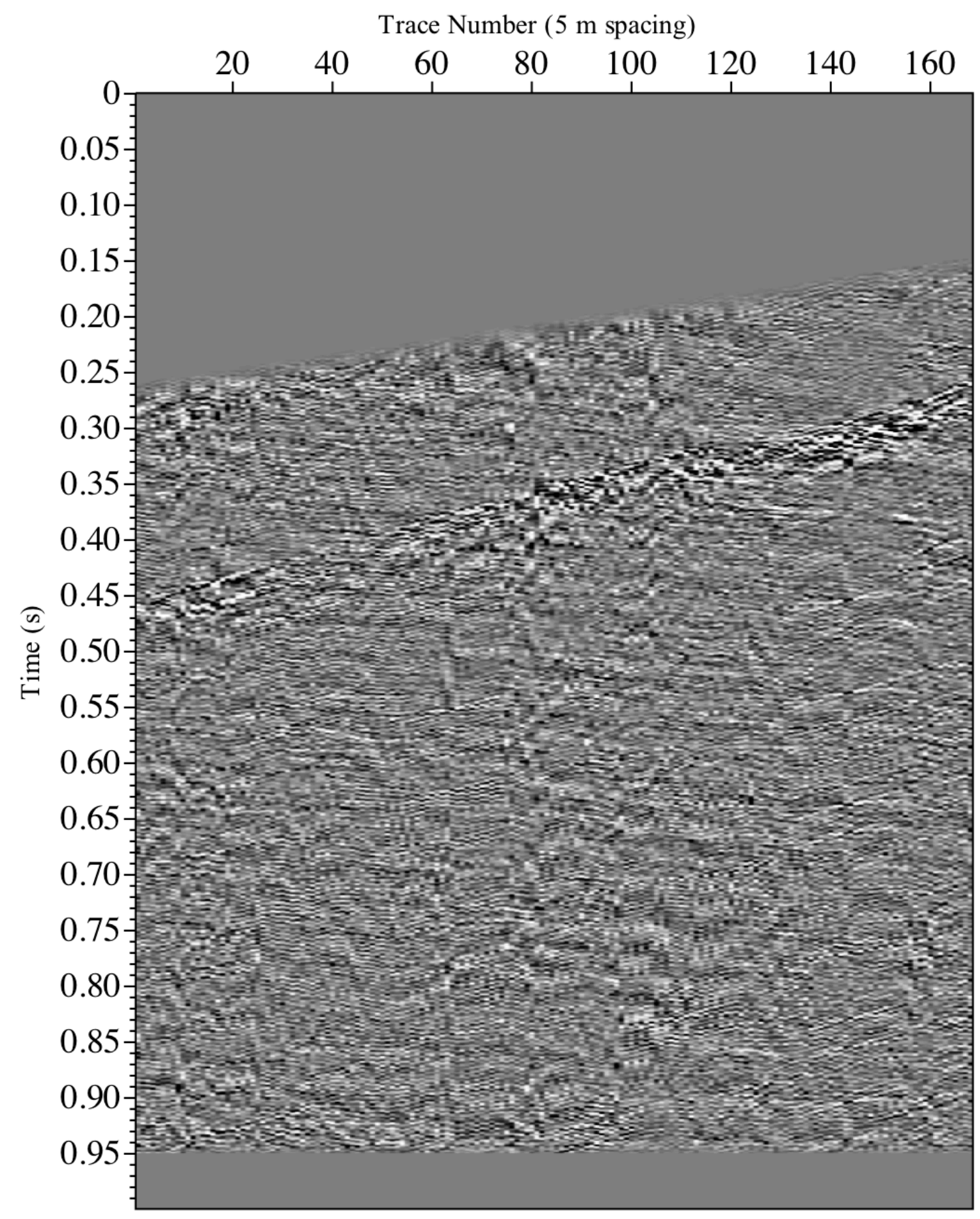

Appendix B.22 - Processed far-offset dynamite source vertical component VSP data from FFS039. 


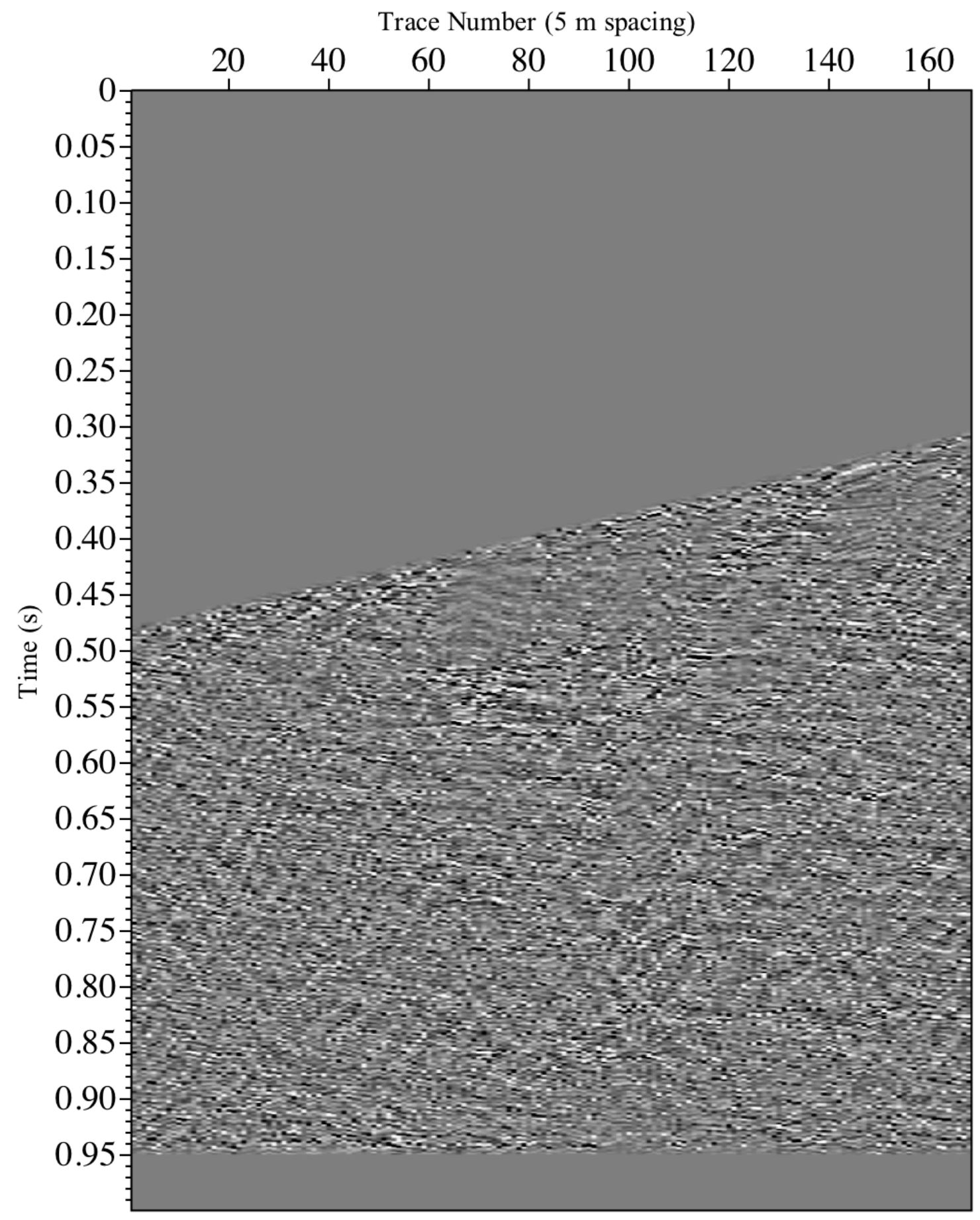

Appendix B.23 - Processed far-offset dynamite source transverse component VSP data from FFS039. 


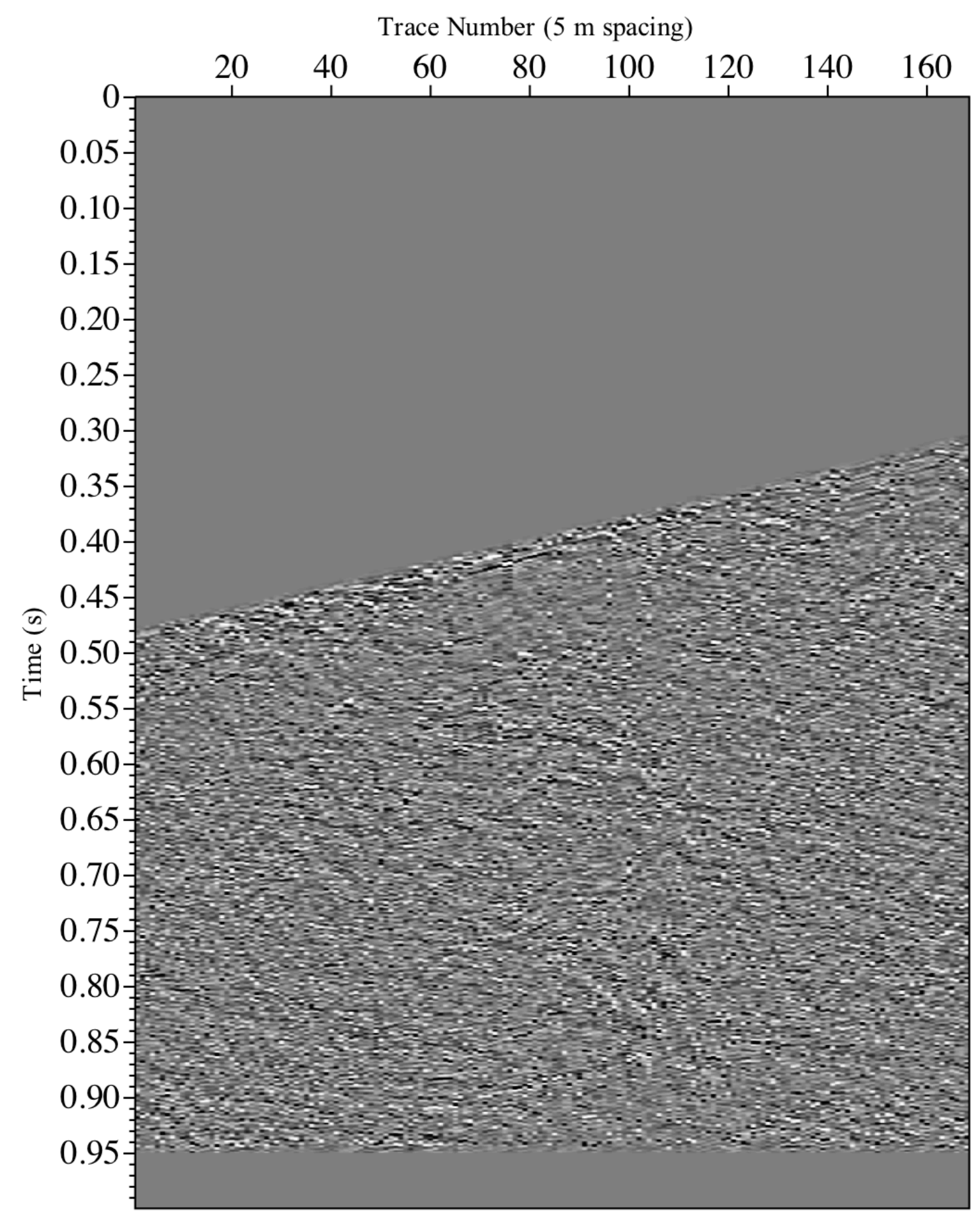

Appendix B.24 - Processed far-offset dynamite source radial component VSP data from FFS039. 


\section{Appendix C - All synthetic VSP data from 3D finite difference modeling}

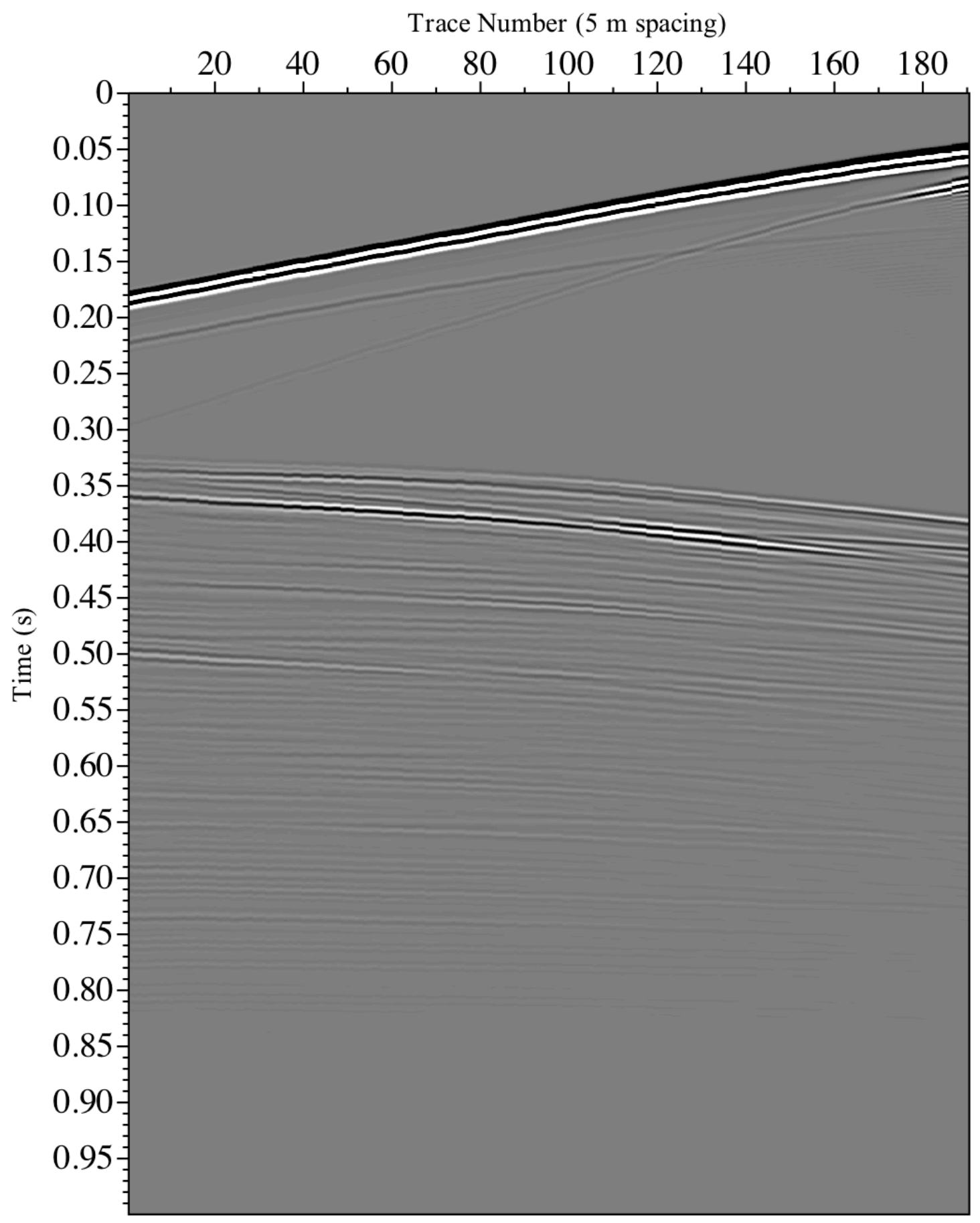

Appendix C.1 - Zero-offset divergence synthetic VSP data from 4Q66W3 in the

'Binary' model. 


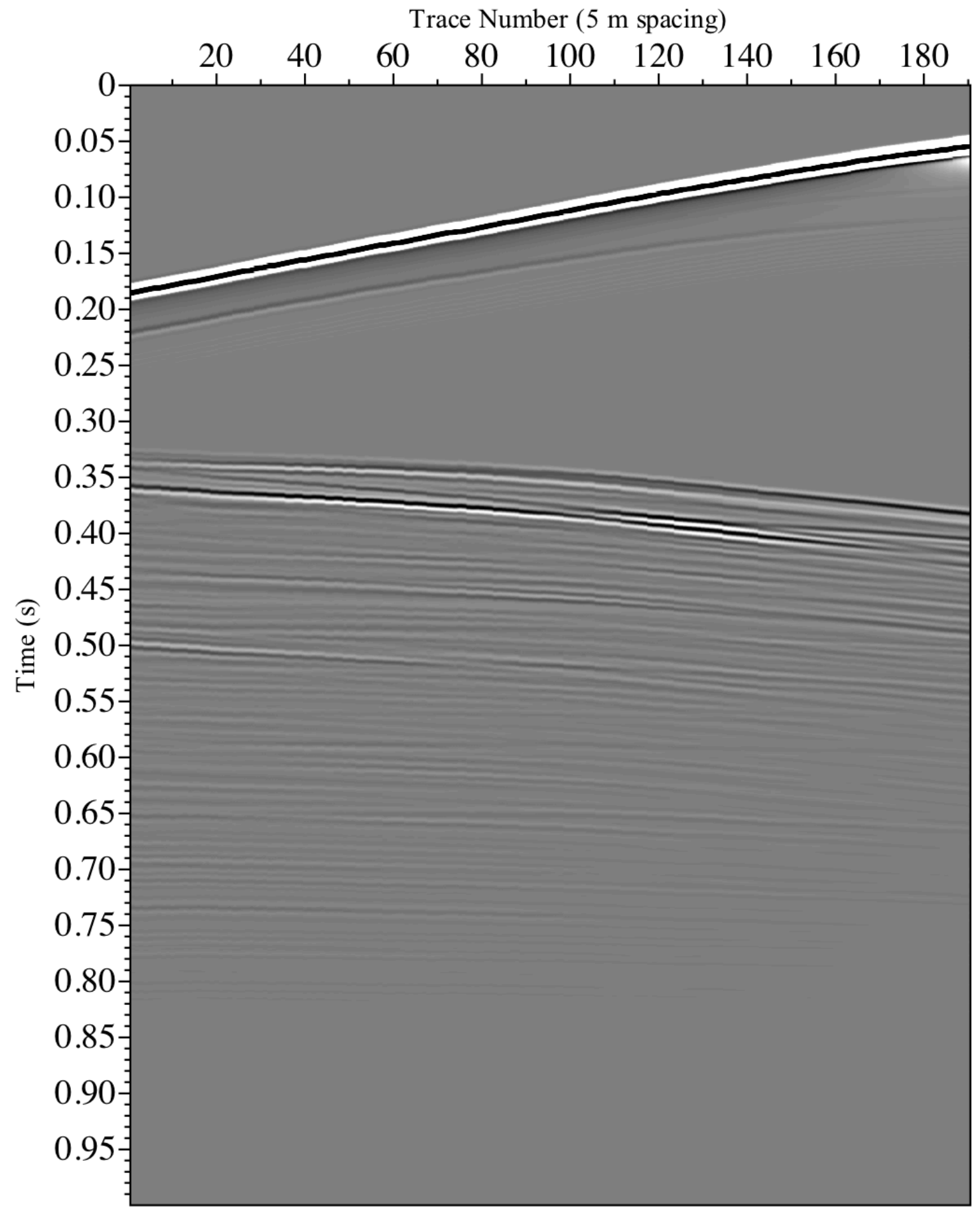

Appendix C.2 - Zero-offset pressure synthetic VSP data from 4Q66W3 in the 'Binary' model. 


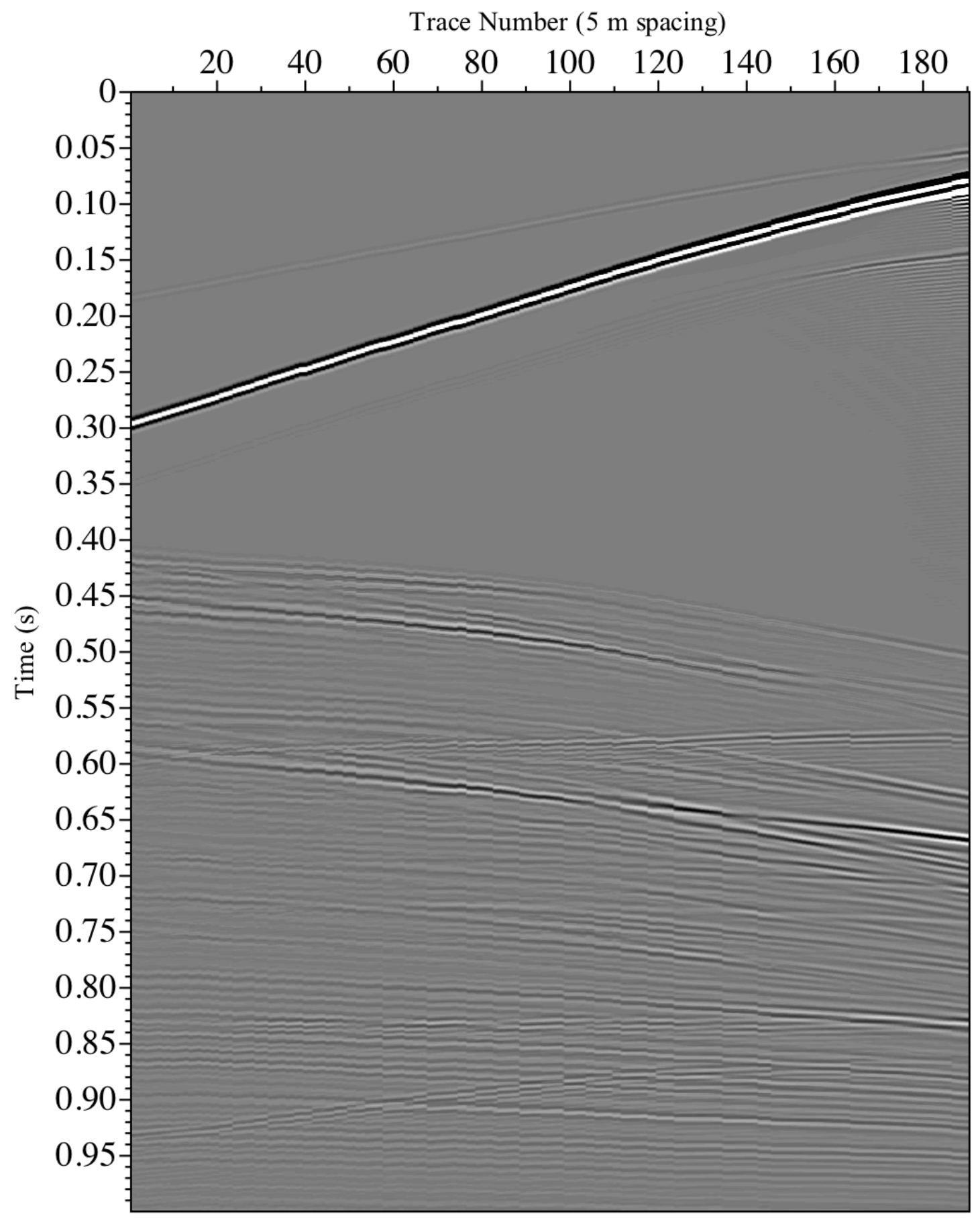

Appendix C.3 - Zero-offset rotation synthetic VSP data from 4Q66W3 in the 'Binary' model. 


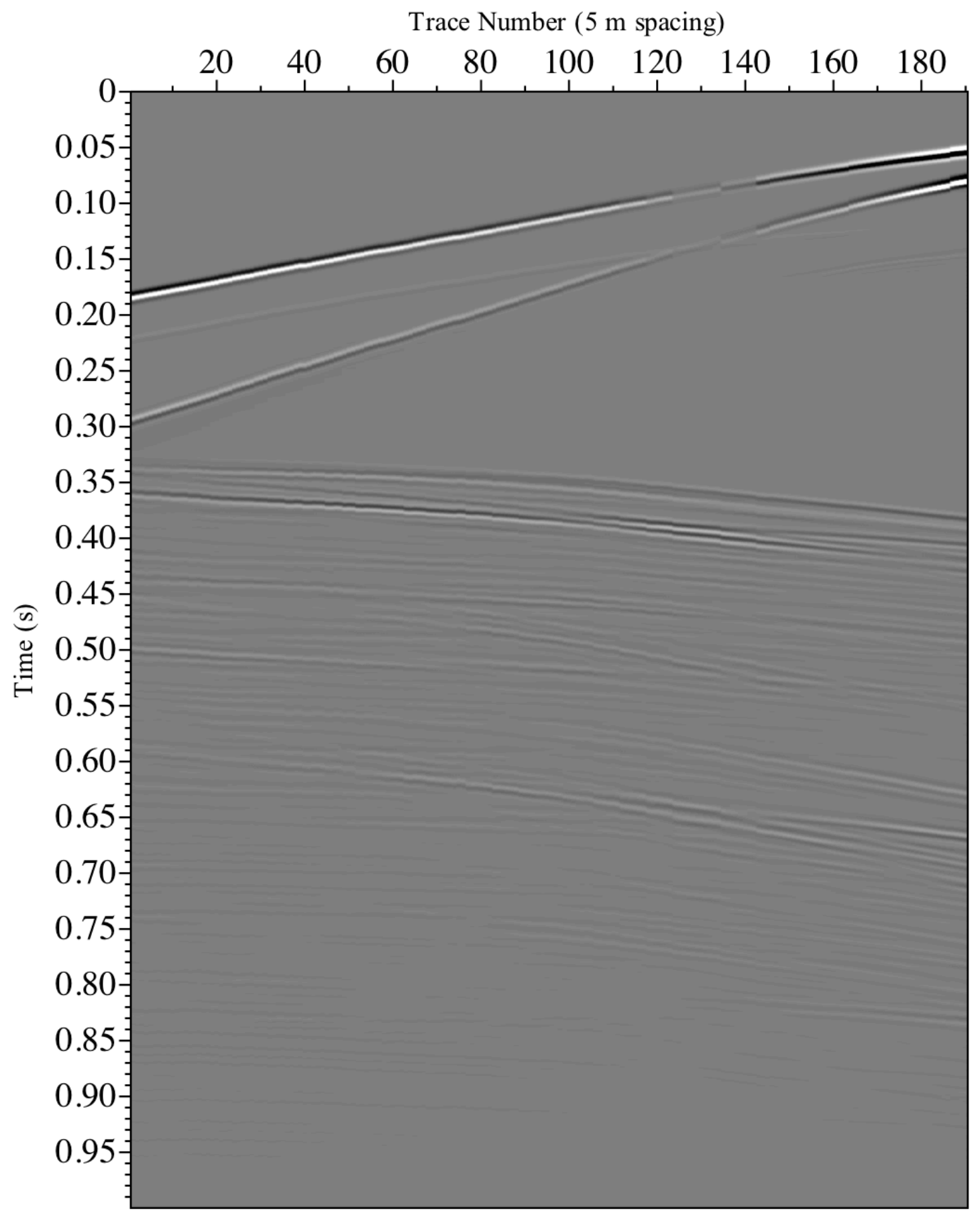

Appendix C.4 - Zero-offset easting particle velocity synthetic VSP data from 4Q66W3 in the 'Binary' model. 


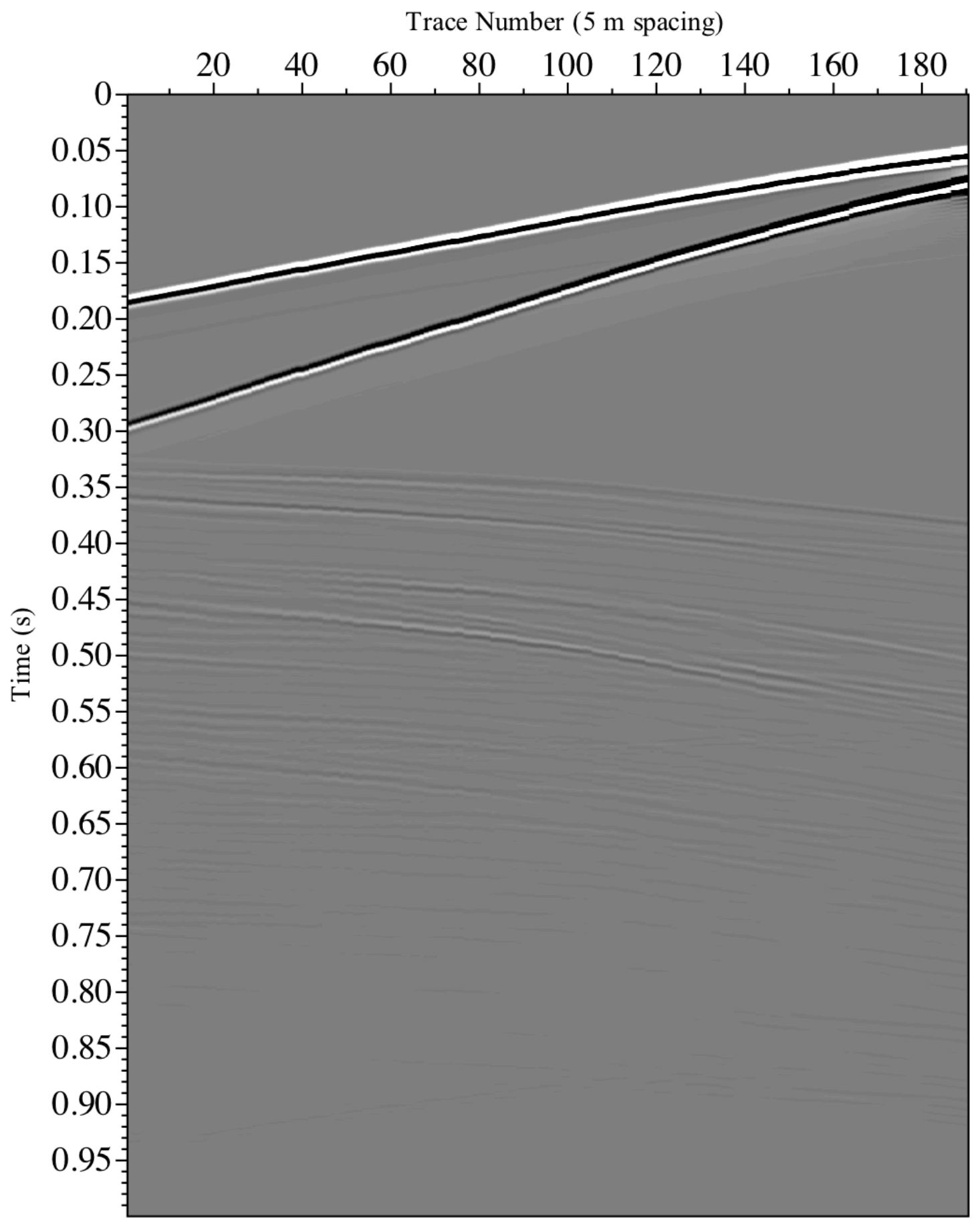

Appendix C.5 - Zero-offset northing particle velocity synthetic VSP data from 4Q66W3 in the 'Binary' model. 


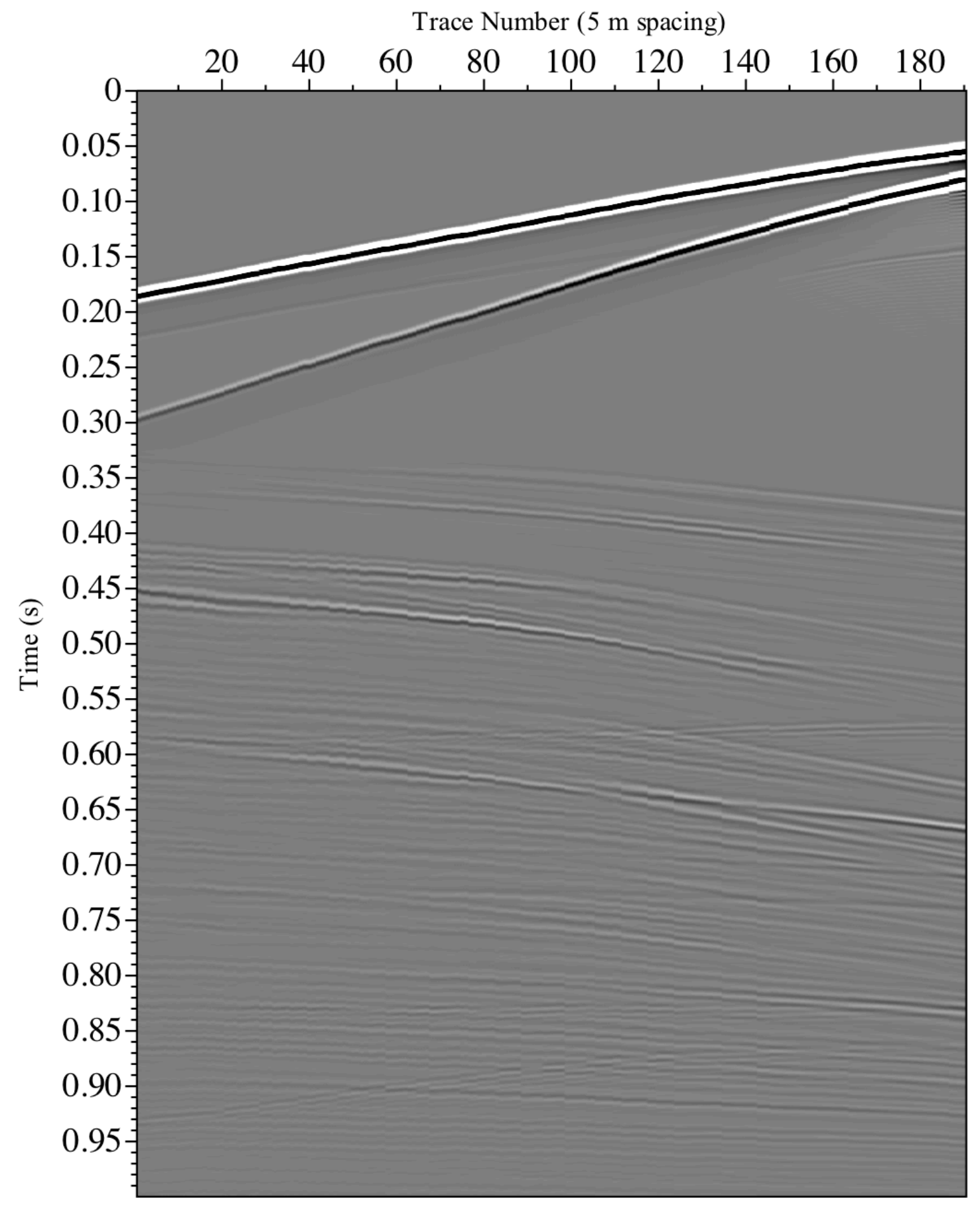

Appendix C.6 - Zero-offset vertical particle velocity synthetic VSP data from 4Q66W3 in the 'Binary' model. 


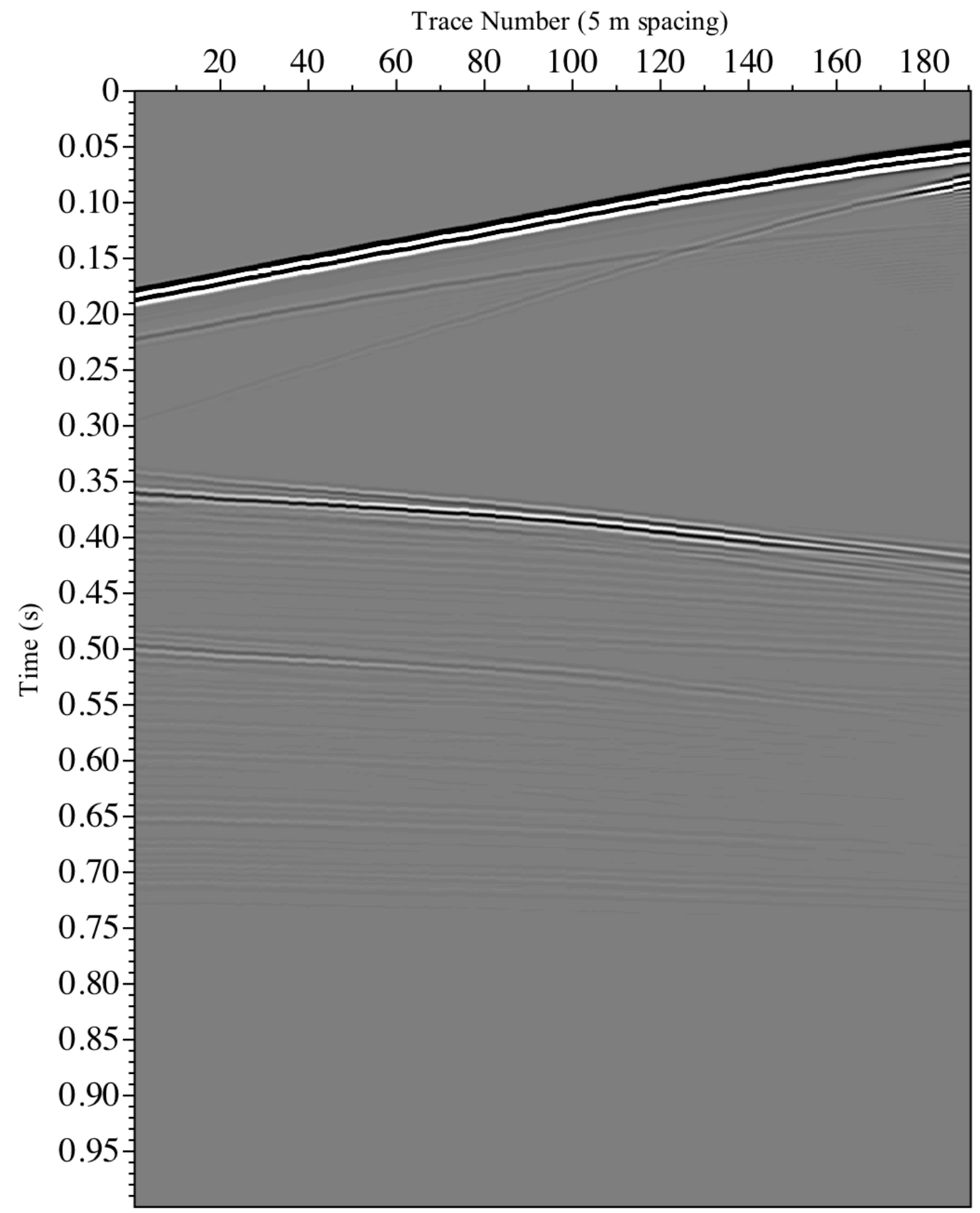

Appendix C.7 - Zero-offset divergence synthetic VSP data from 4Q66W3 in the 'Binary

(Callinan East)' model. 


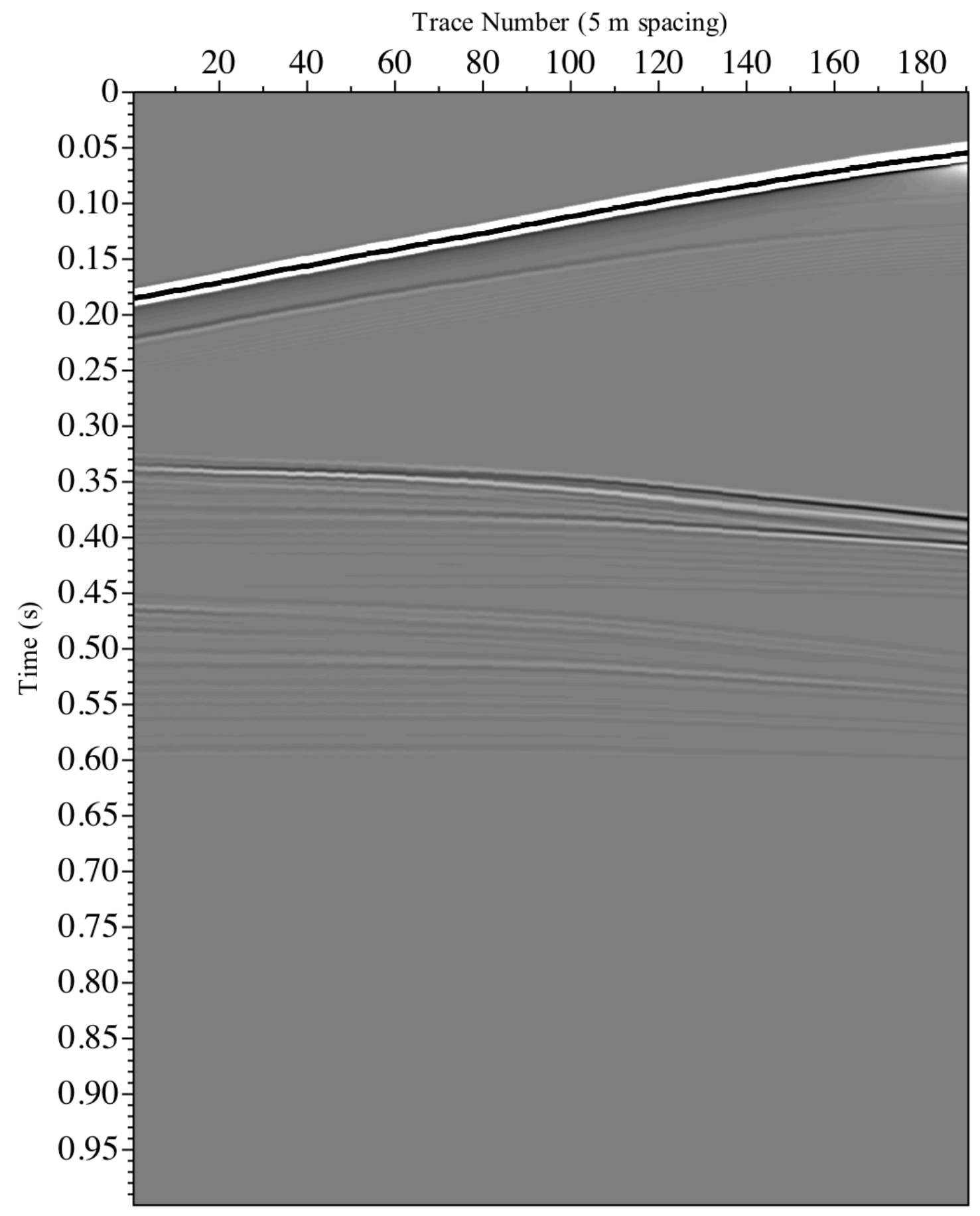

Appendix C.8 - Zero-offset pressure synthetic VSP data from 4Q66W3 in the 'Binary (Callinan East)' model. 


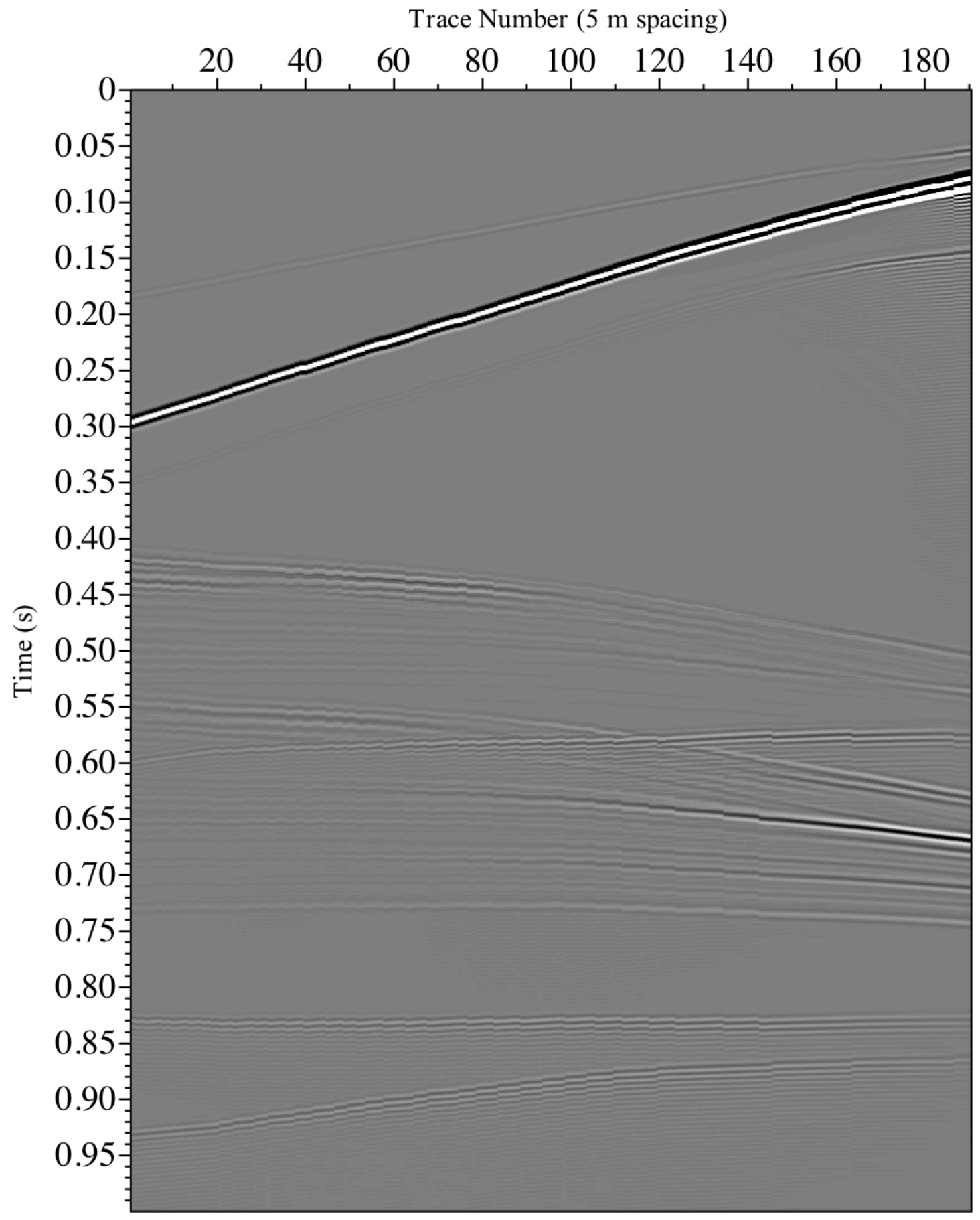

Appendix C.9 - Zero-offset rotation synthetic VSP data from 4Q66W3 in the 'Binary (Callinan East)' model. 


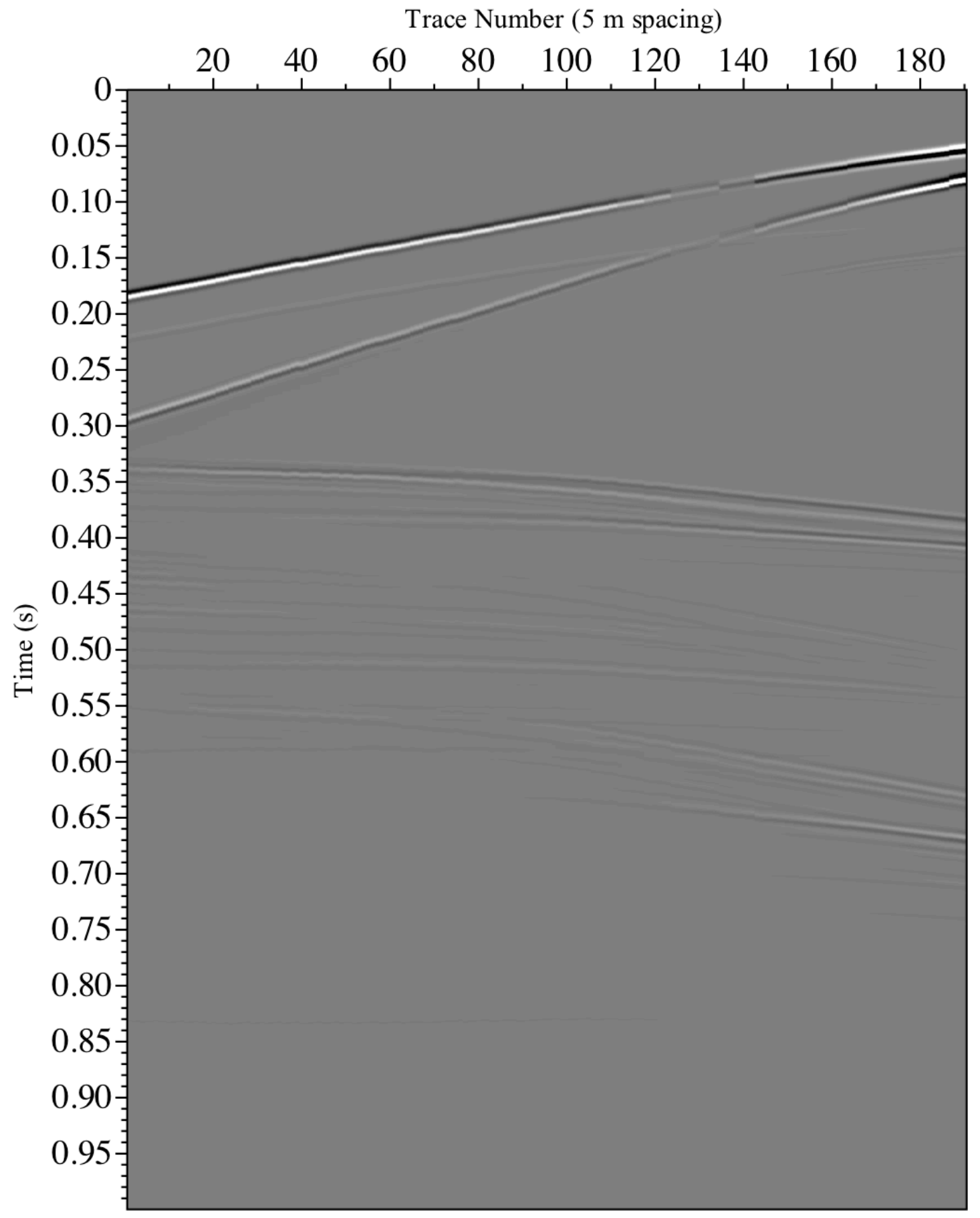

Appendix C.10 - Zero-offset easting particle velocity synthetic VSP data from 4Q66W3 in the 'Binary (Callinan East)' model. 


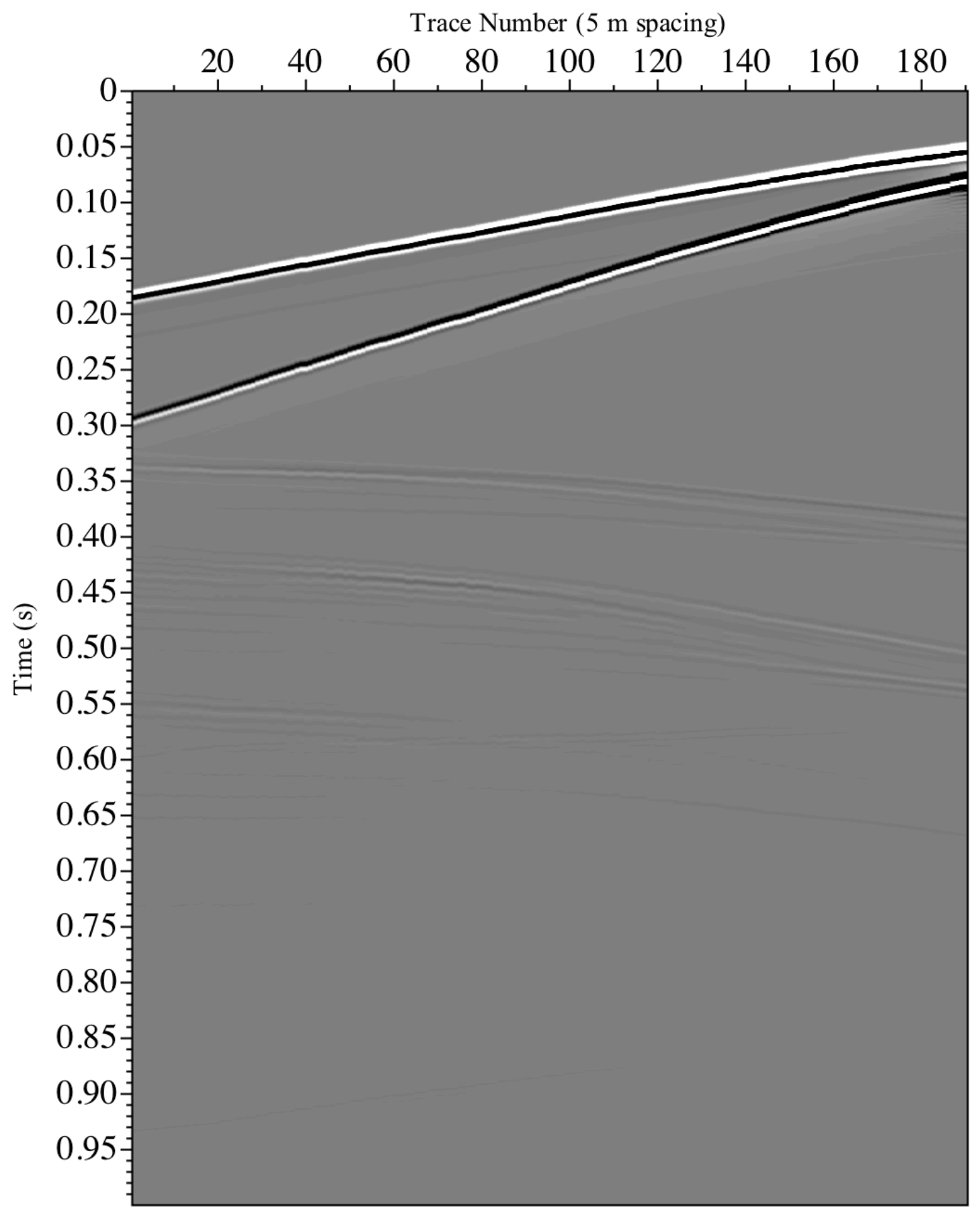

Appendix C.11 - Zero-offset northing particle velocity synthetic VSP data from 4Q66W3 in the 'Binary (Callinan East)' model. 


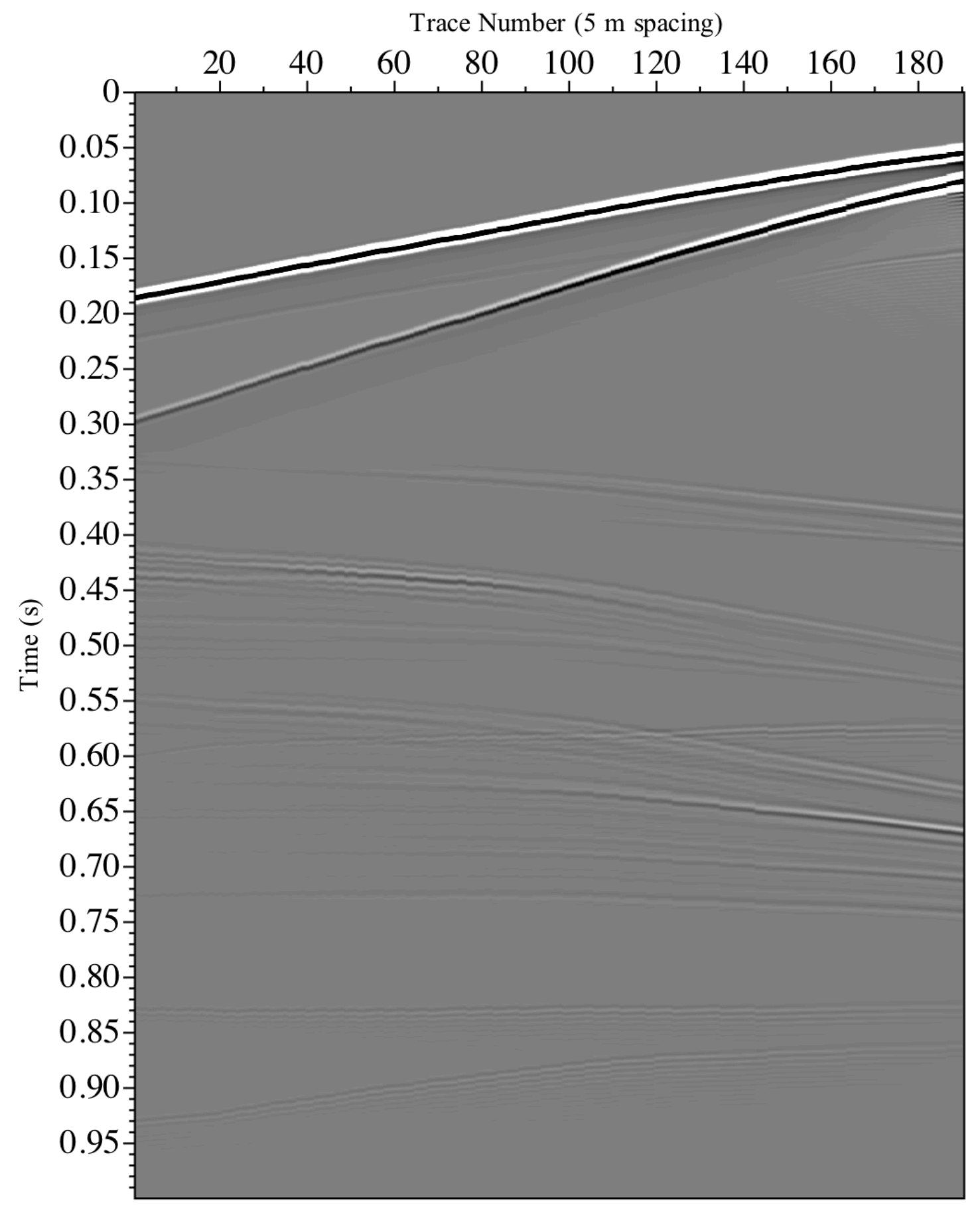

Appendix C.12 - Zero-offset vertical particle velocity synthetic VSP data from 4Q66W3 in the 'Binary (Callinan East)' model. 


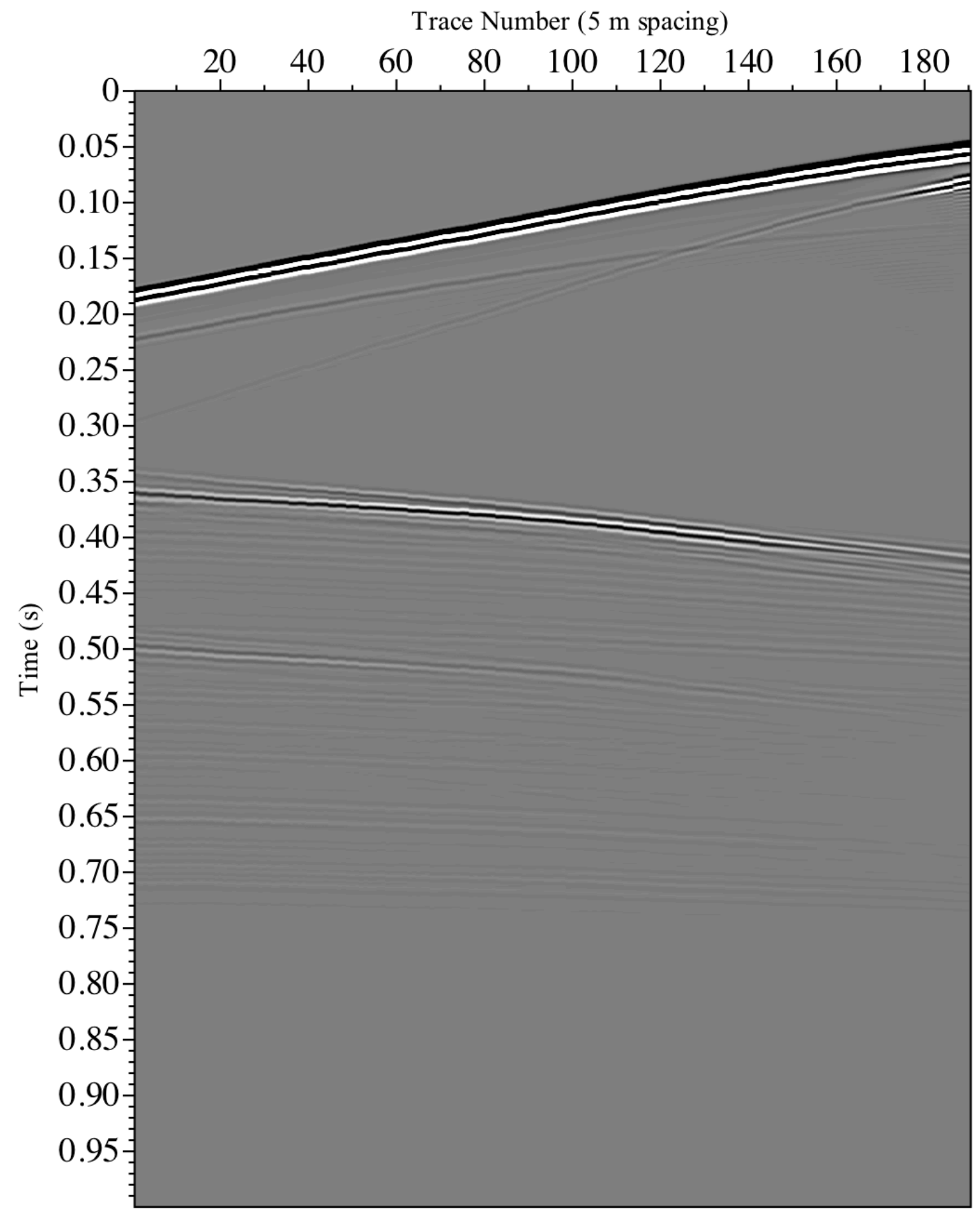

Appendix C.13 - Zero-offset divergence synthetic VSP data from 4Q66W3 in the

'Binary (Callinan North)' model. 


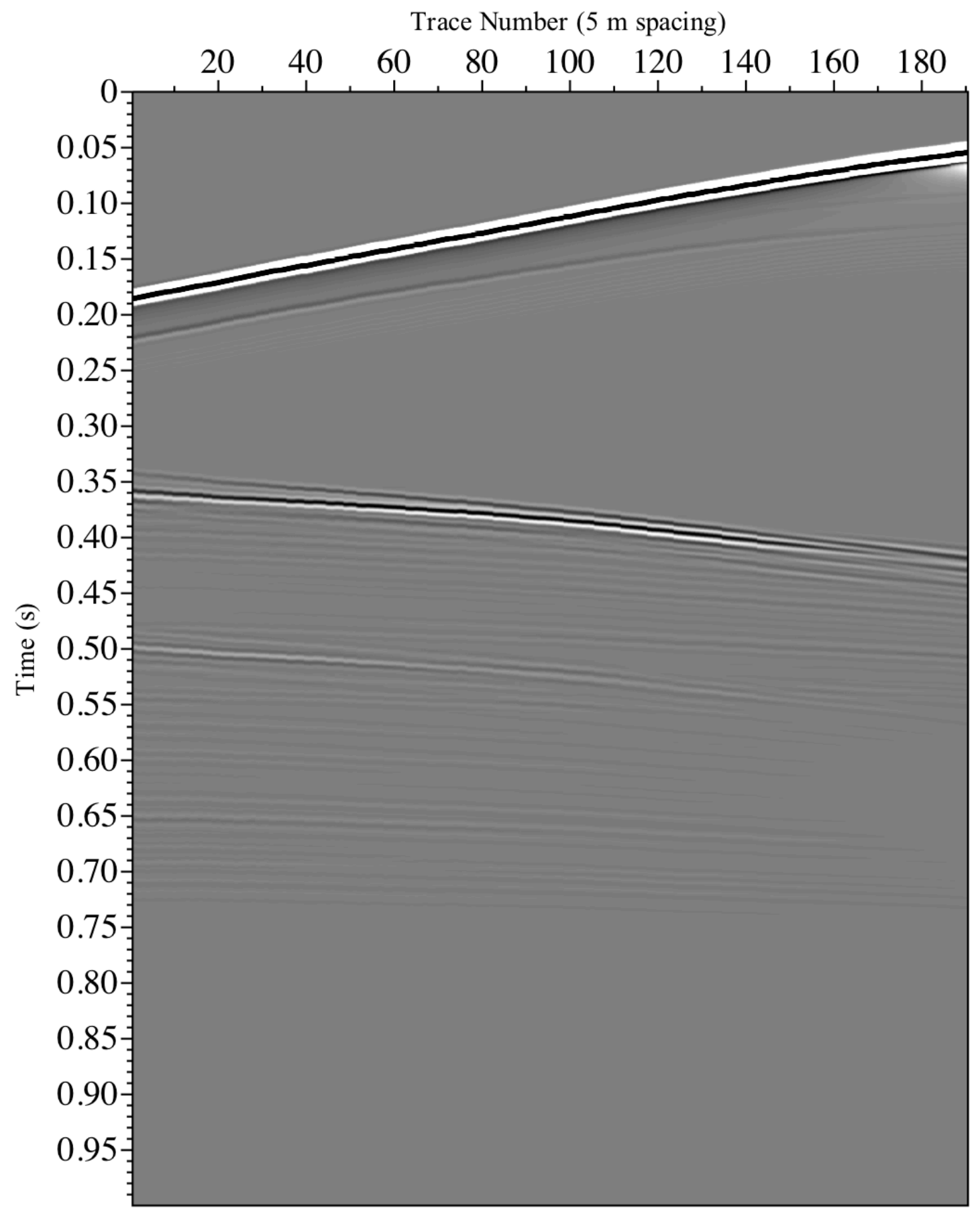

Appendix C.14 - Zero-offset pressure synthetic VSP data from 4Q66W3 in the 'Binary

(Callinan North)' model. 


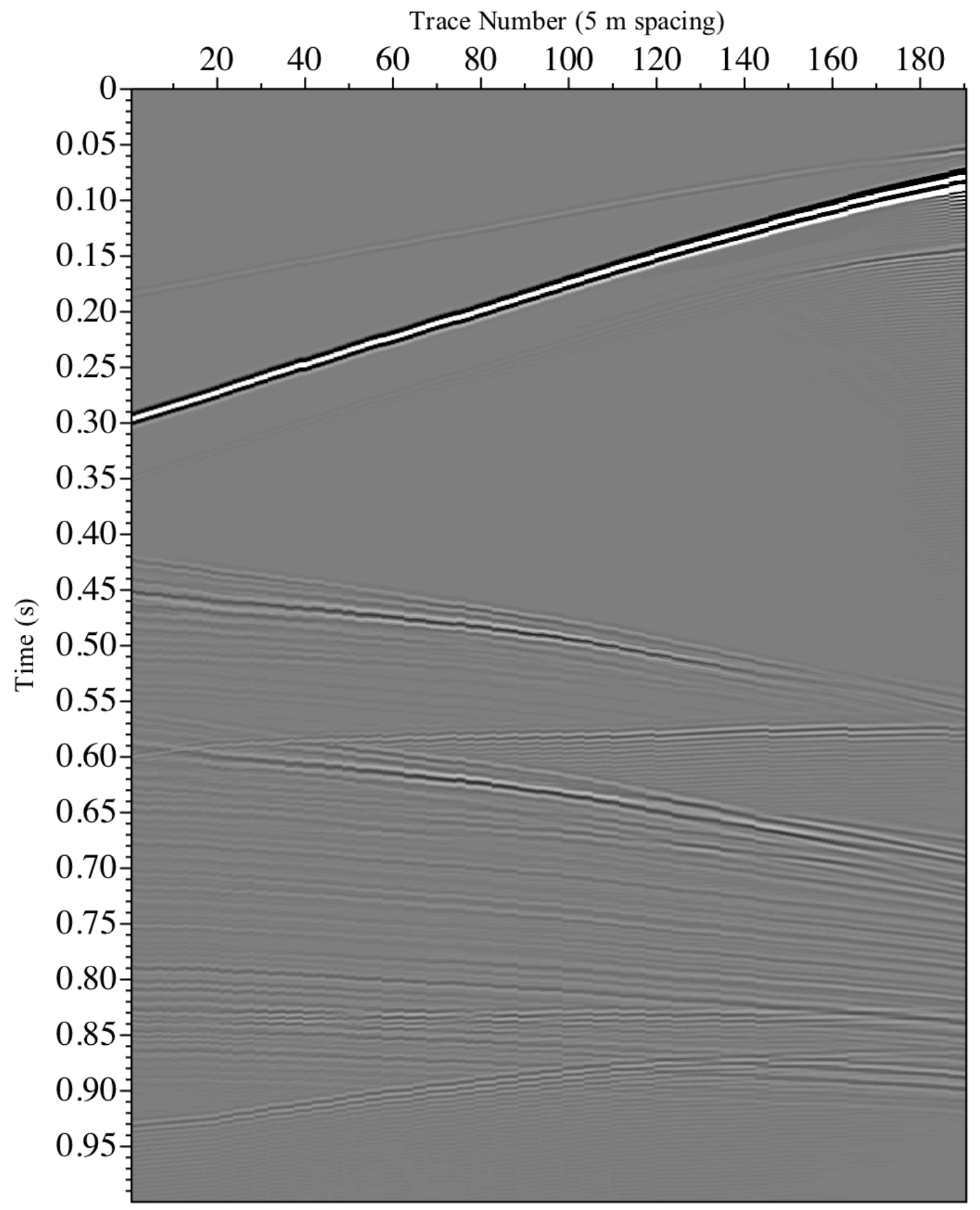

Appendix C.15 - Zero-offset rotation synthetic VSP data from 4Q66W3 in the 'Binary

(Callinan North)' model. 


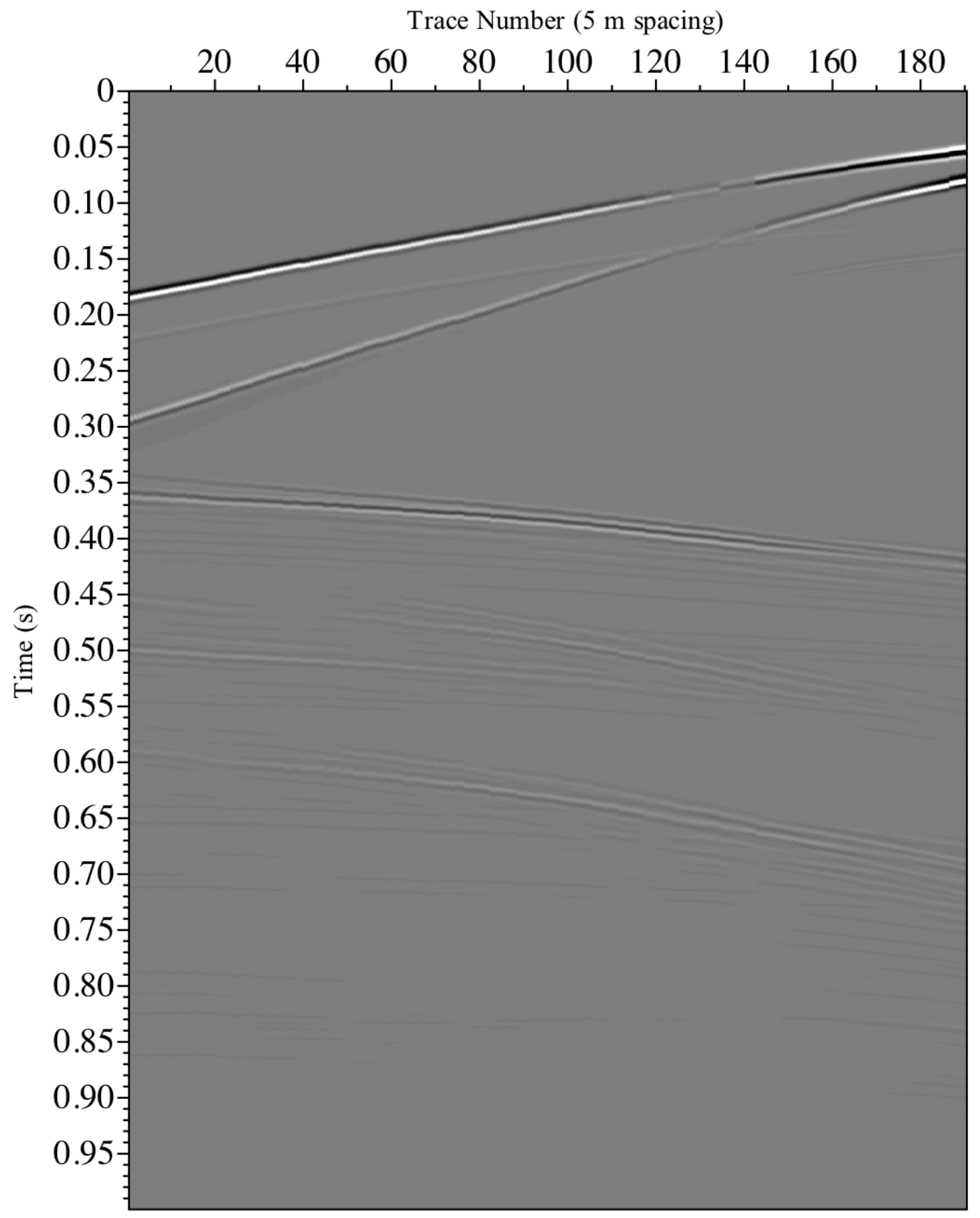

Appendix C.16 - Zero-offset easting particle velocity synthetic VSP data from 4Q66W3 in the 'Binary (Callinan North)' model. 


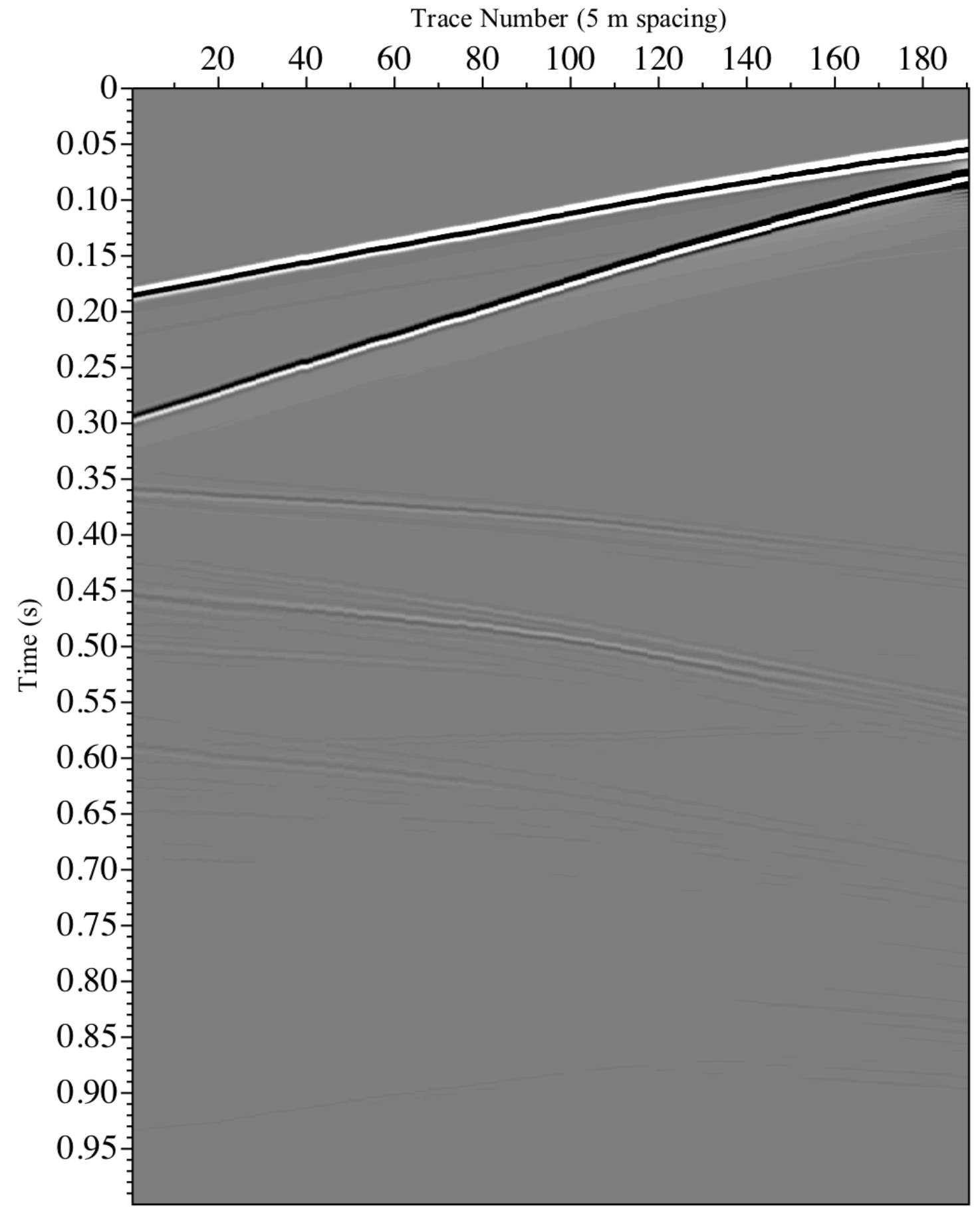

Appendix C.17 - Zero-offset northing particle velocity synthetic VSP data from 4Q66W3 in the 'Binary (Callinan North)' model. 


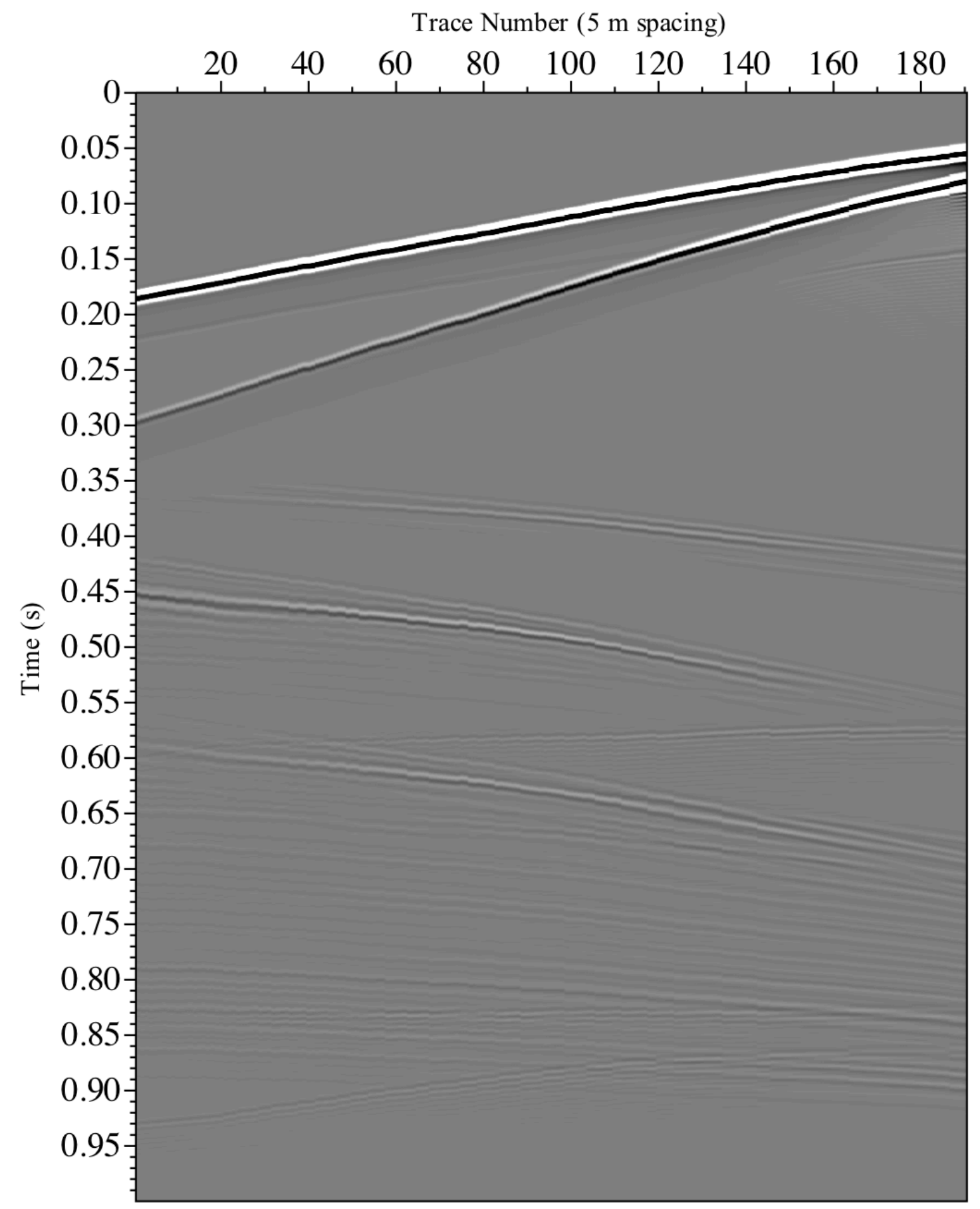

Appendix C.18 - Zero-offset vertical particle velocity synthetic VSP data from 4Q66W3 in the 'Binary (Callinan North)' model. 


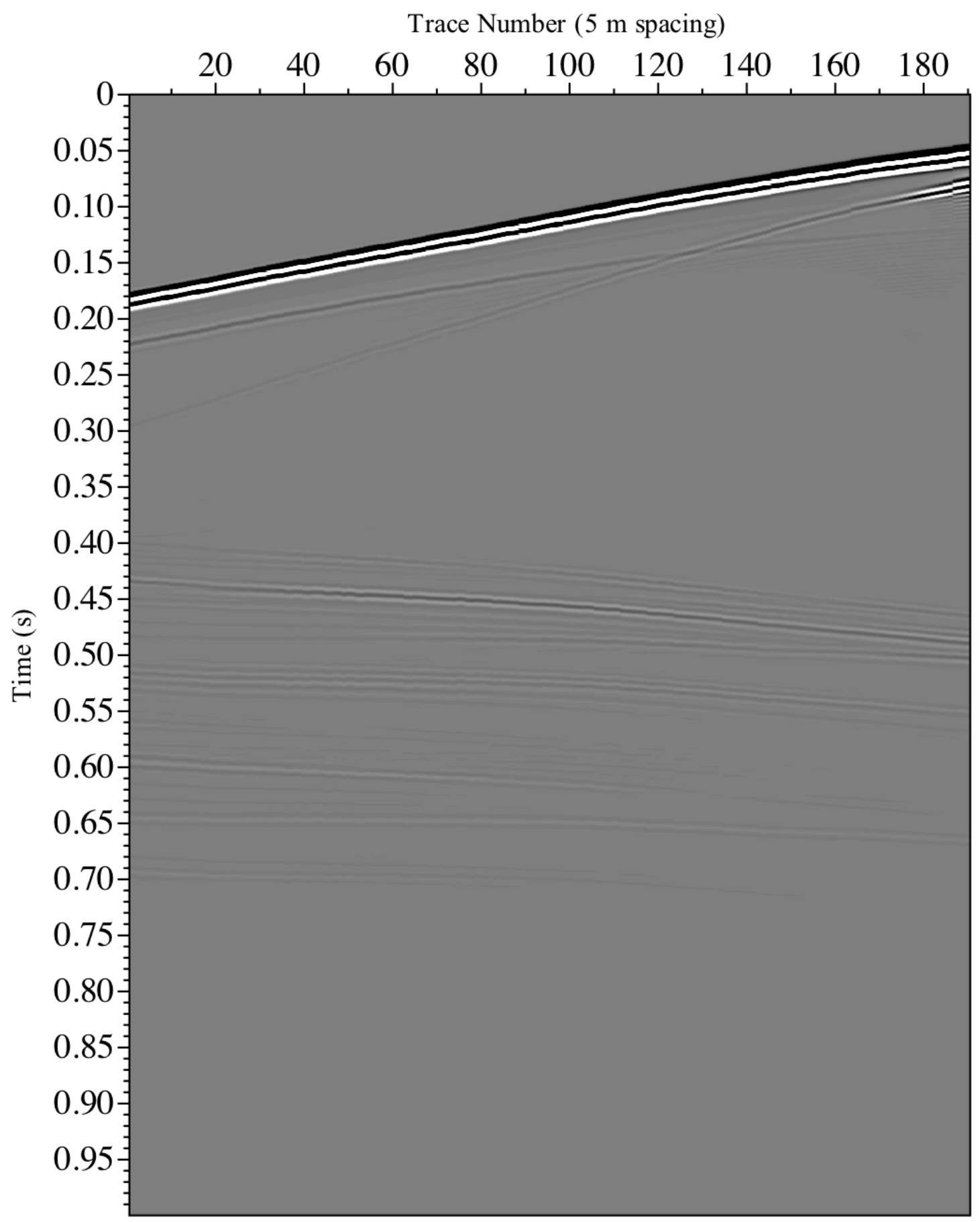

Appendix C.19 - Zero-offset divergence synthetic VSP data from 4Q66W3 in the

'Binary (Callinan South)' model. 


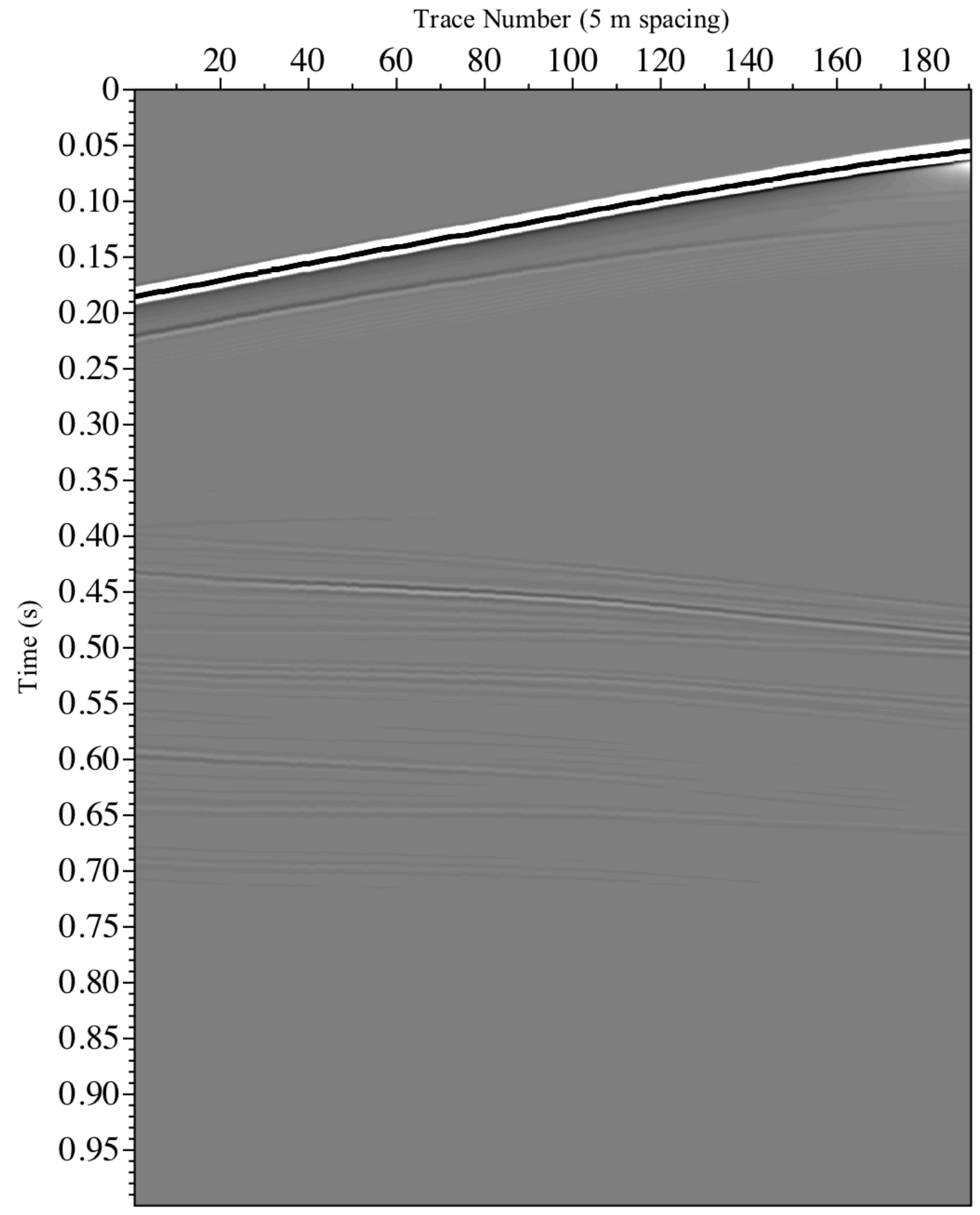

Appendix C.20 - Zero-offset pressure synthetic VSP data from 4Q66W3 in the 'Binary

(Callinan South)' model. 


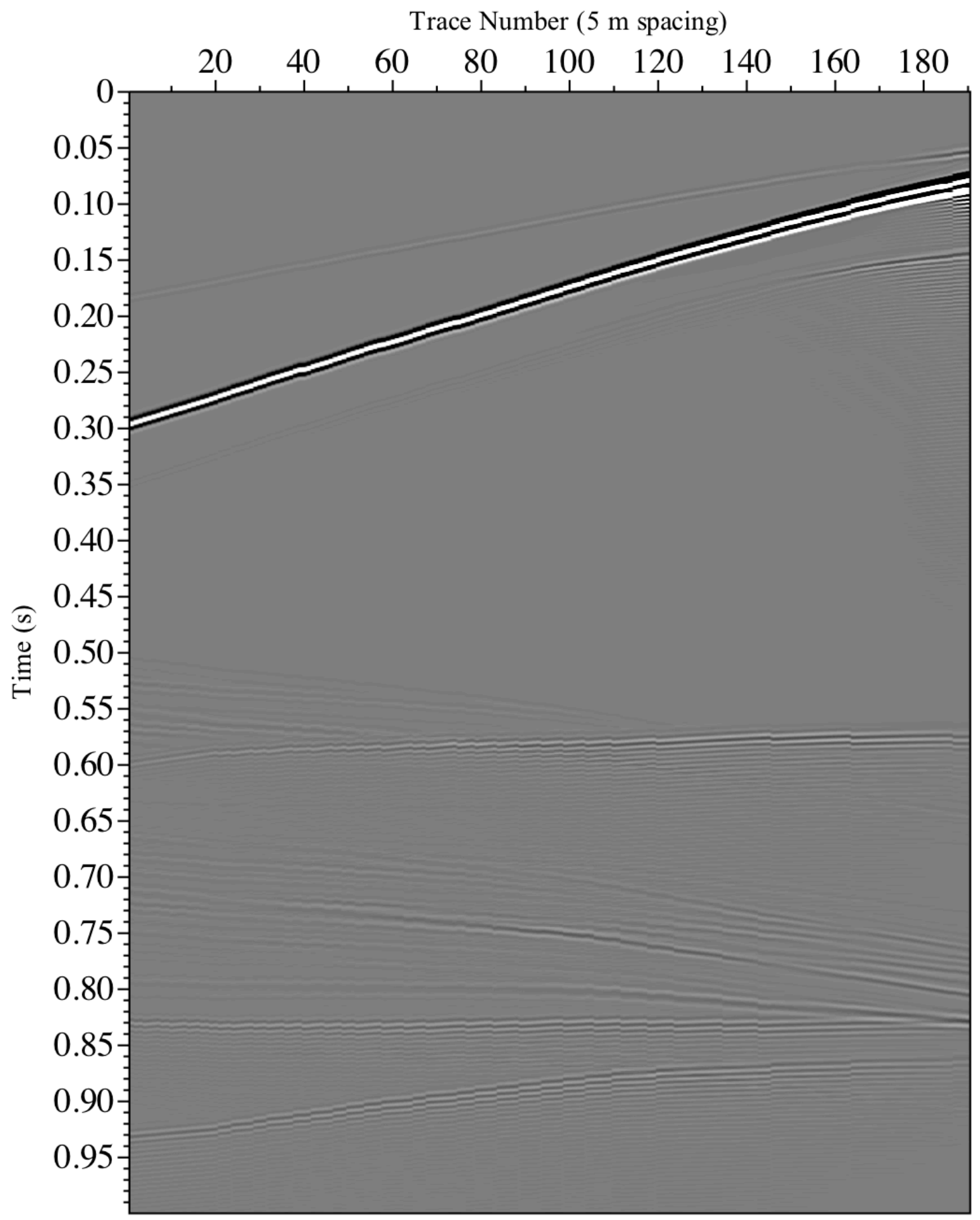

Appendix C.21 - Zero-offset rotation synthetic VSP data from 4Q66W3 in the 'Binary (Callinan South)' model. 


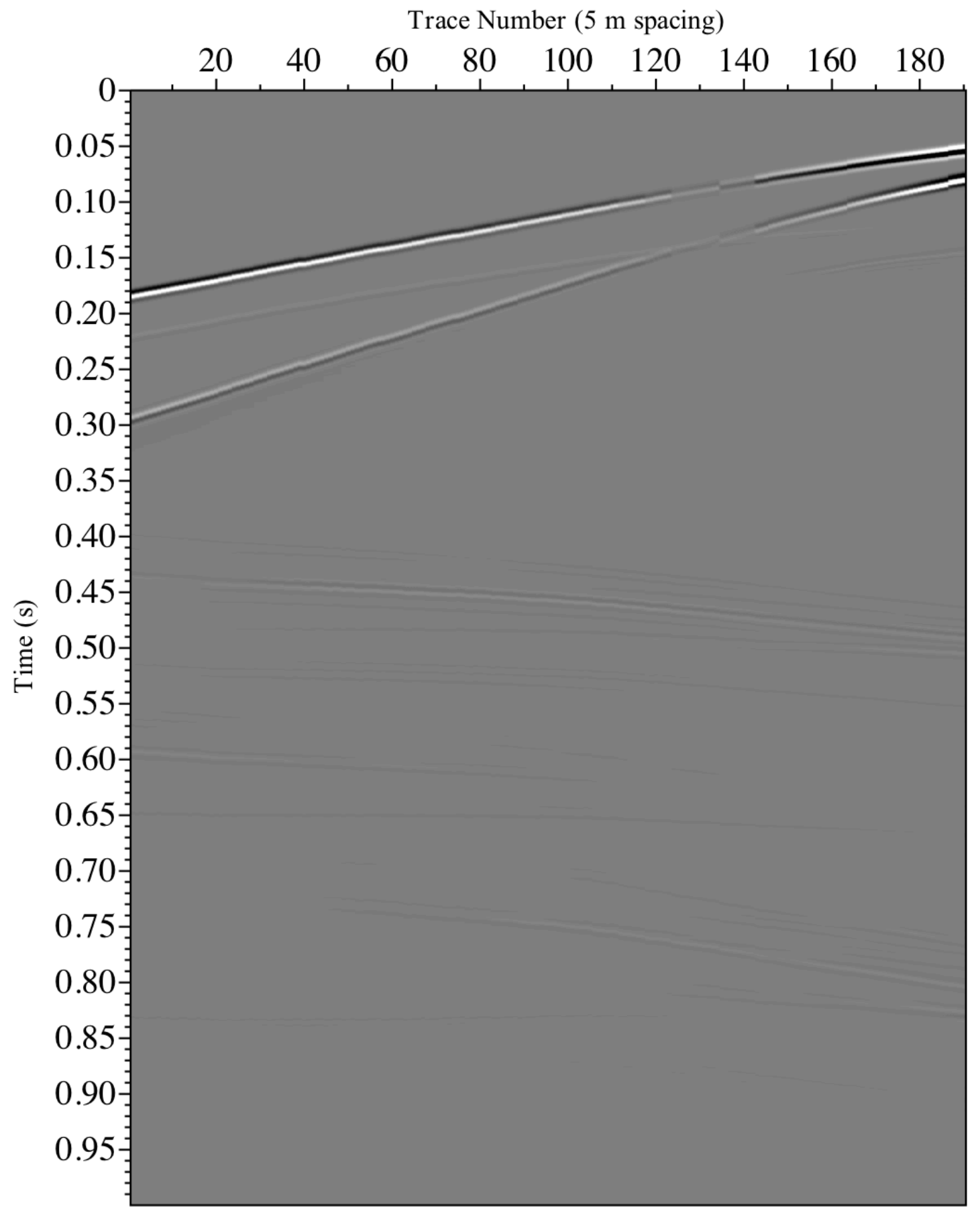

Appendix C.22 - Zero-offset easting particle velocity synthetic VSP data from 4Q66W3 in the 'Binary (Callinan South)' model. 


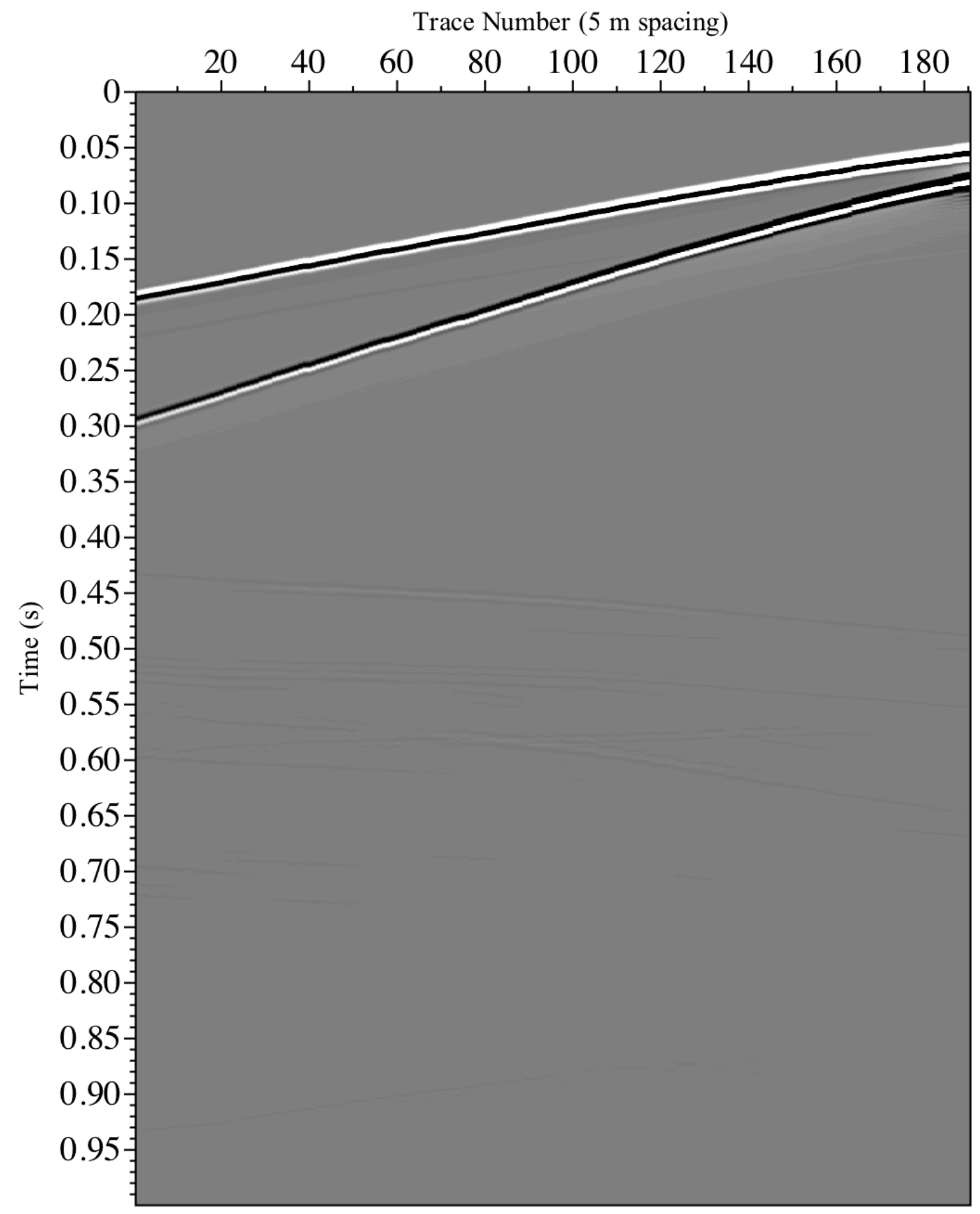

Appendix C.23 - Zero-offset northing particle velocity synthetic VSP data from 4Q66W3 in the 'Binary (Callinan South)' model. 


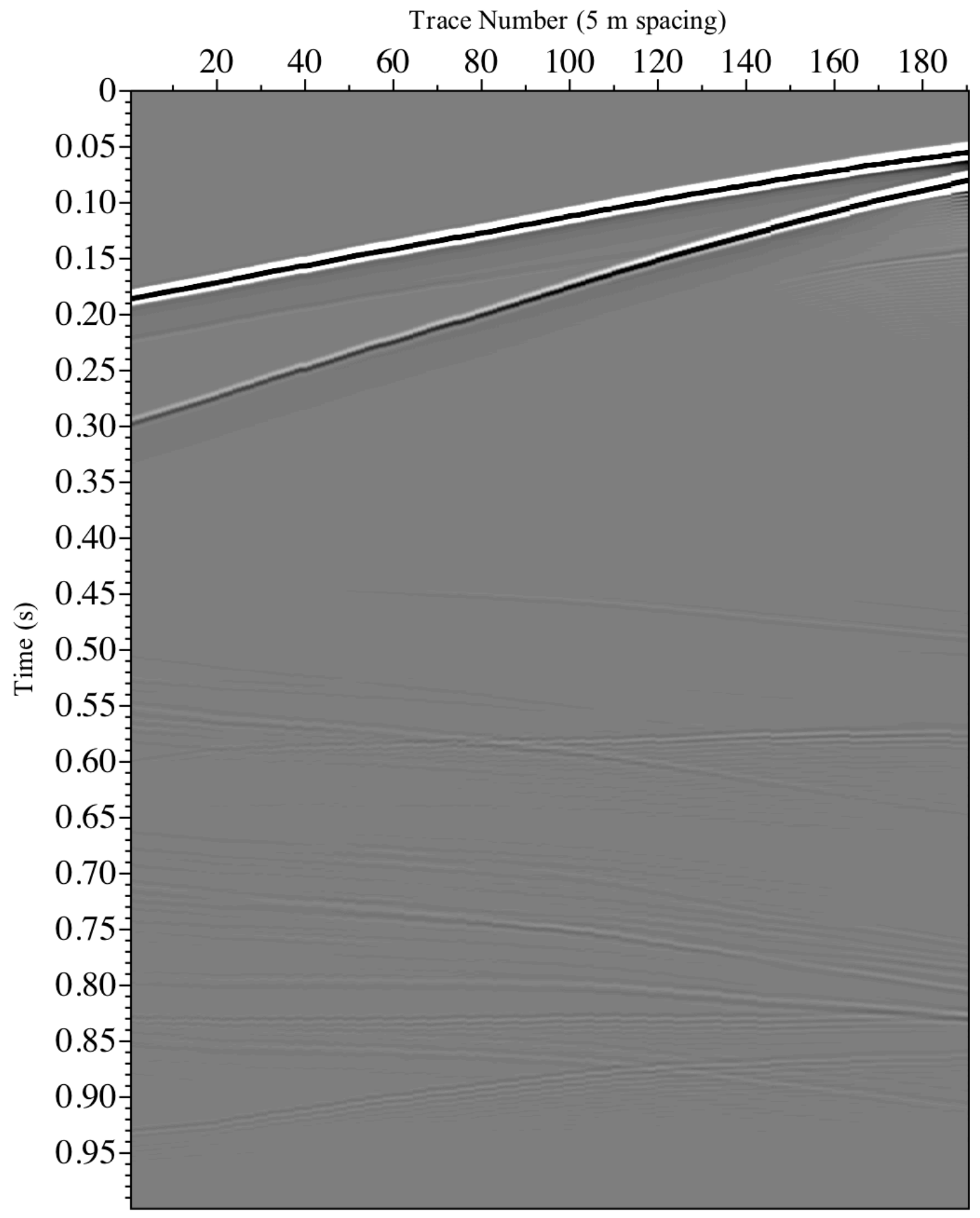

Appendix C.24 - Zero-offset vertical particle velocity synthetic VSP data from 4Q66W3 in the 'Binary (Callinan South)' model. 


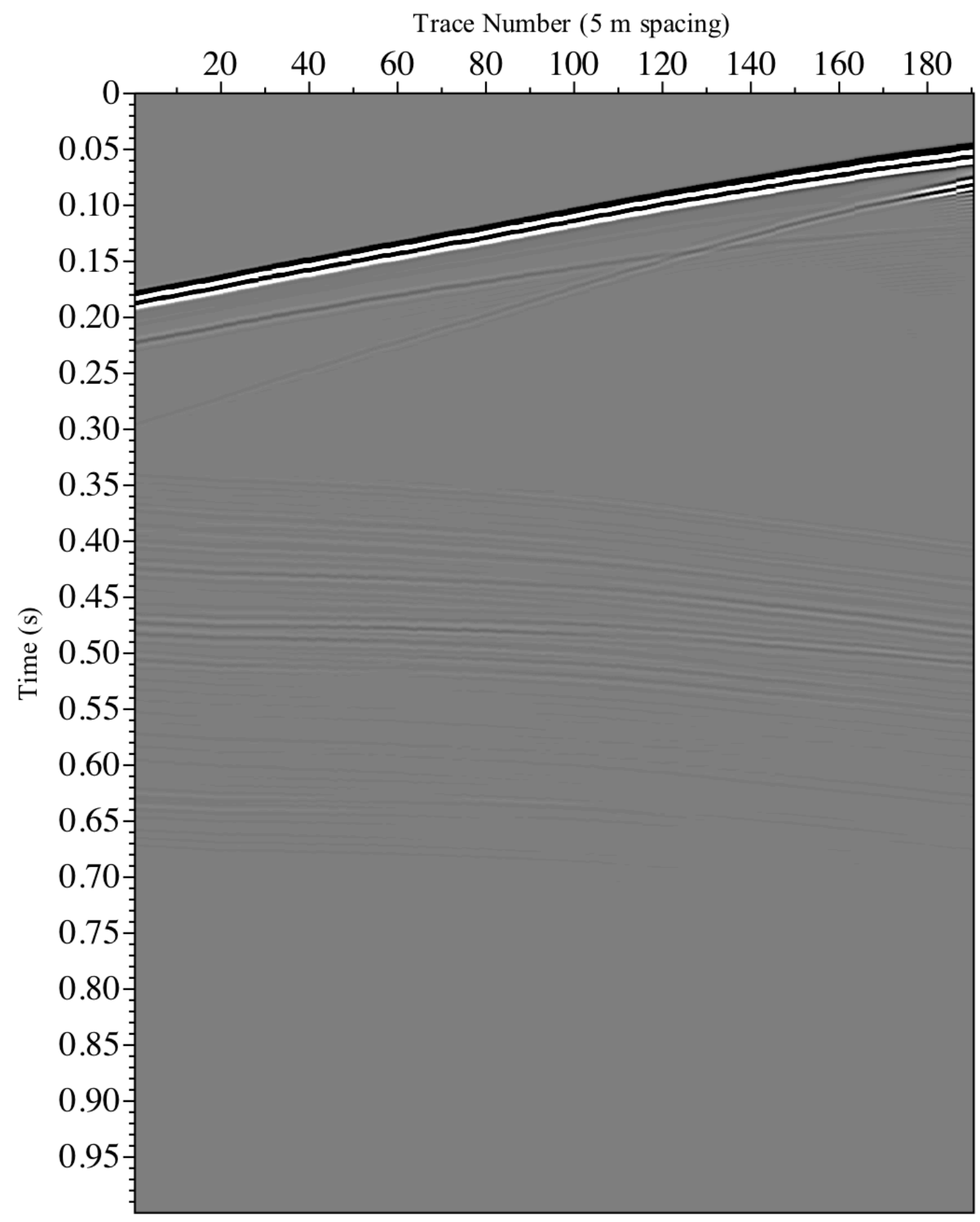

Appendix C.25 - Zero-offset divergence synthetic VSP data from 4Q66W3 in the

'Binary (777)' model. 


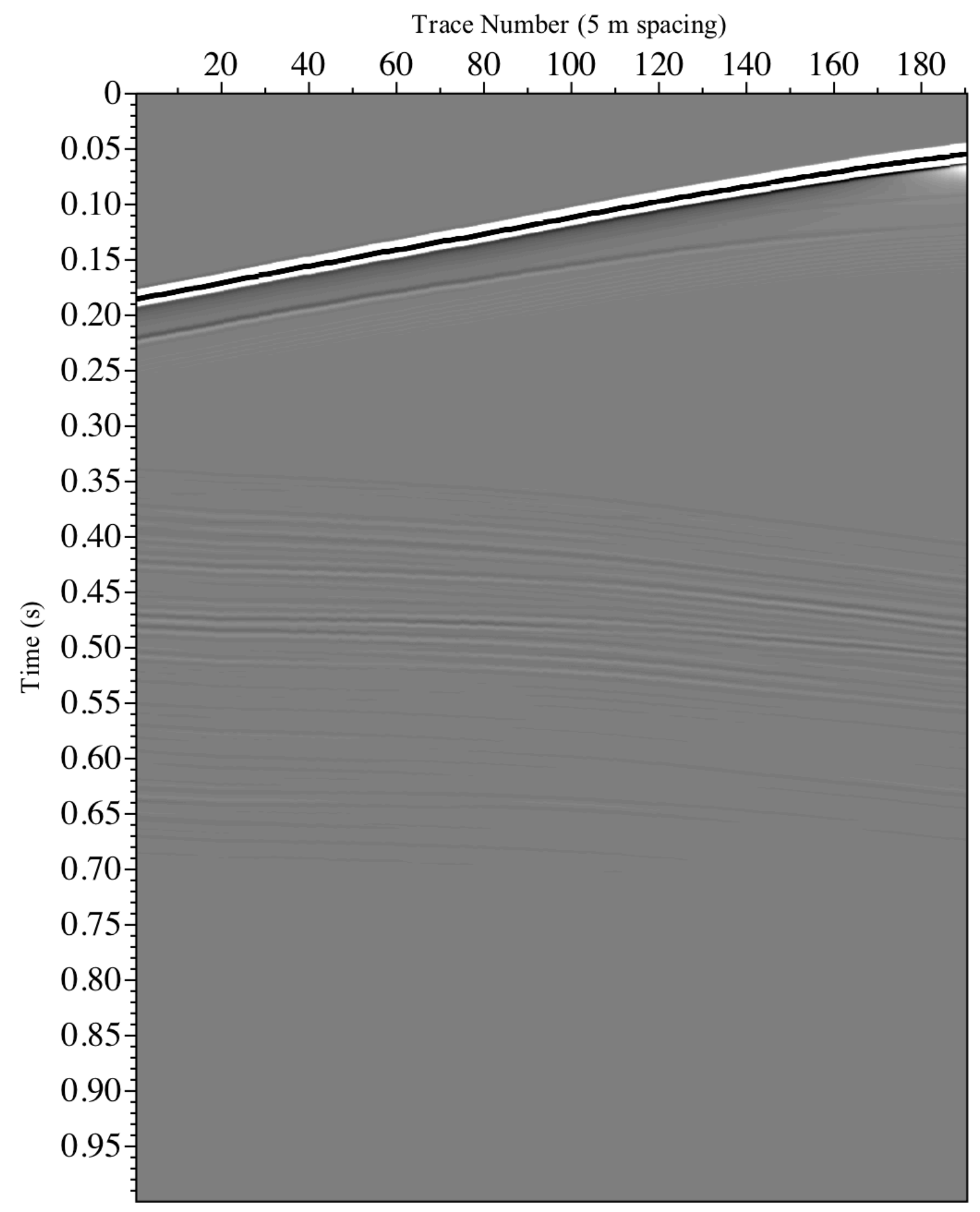

Appendix C.26 - Zero-offset pressure synthetic VSP data from 4Q66W3 in the 'Binary (777)' model. 


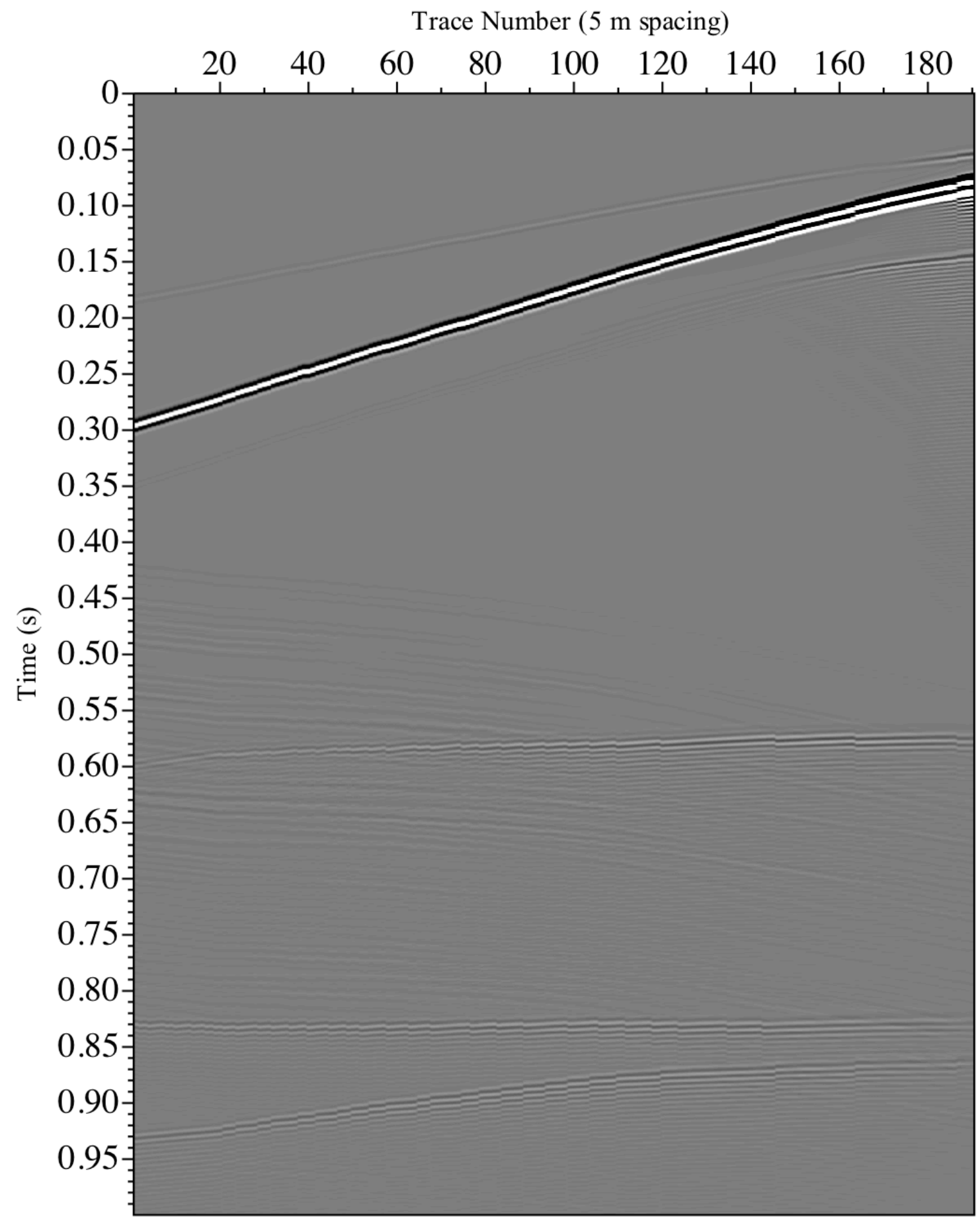

Appendix C.27 - Zero-offset rotation synthetic VSP data from 4Q66W3 in the 'Binary (777)' model. 


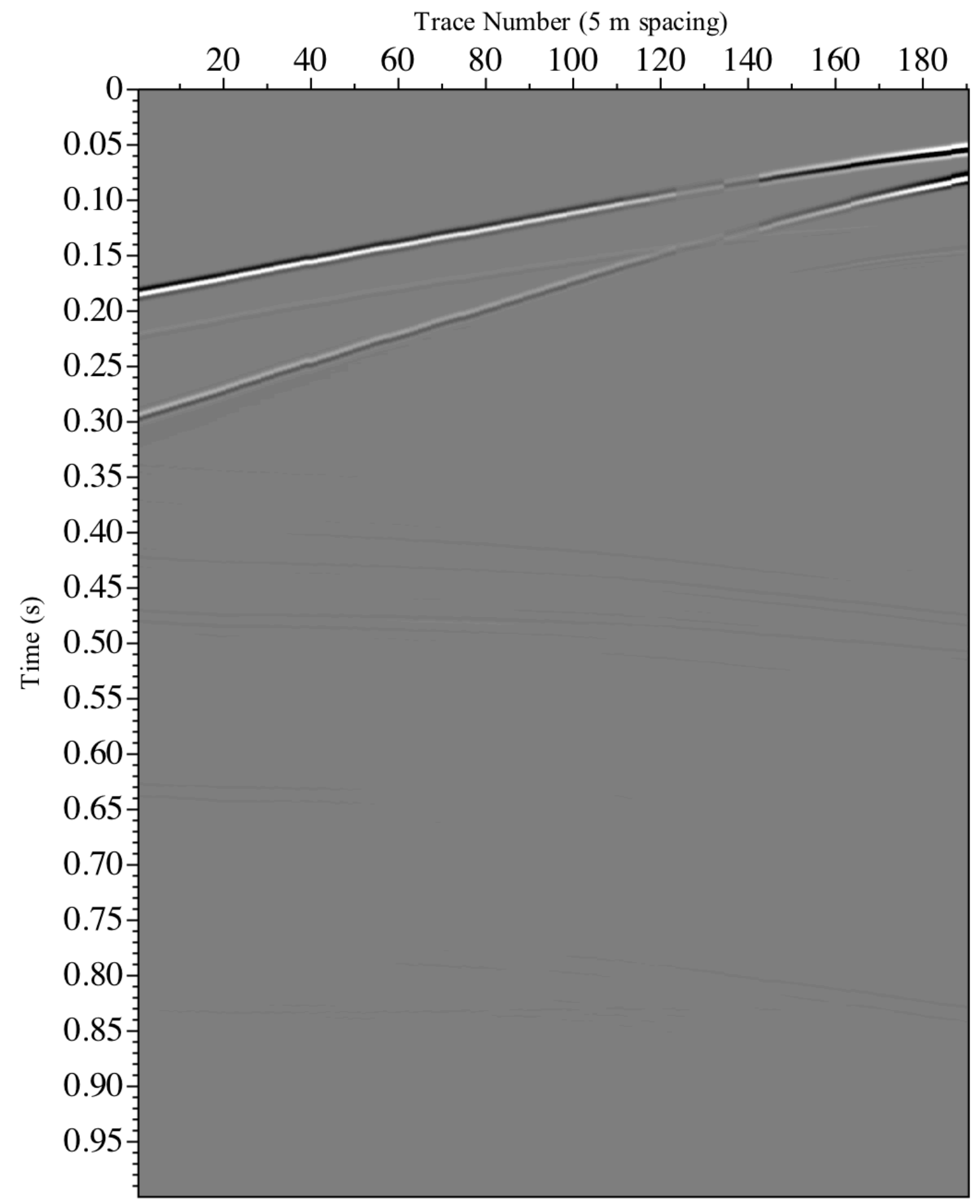

Appendix C.28 - Zero-offset easting particle velocity synthetic VSP data from 4Q66W3

in the 'Binary (777)' model. 


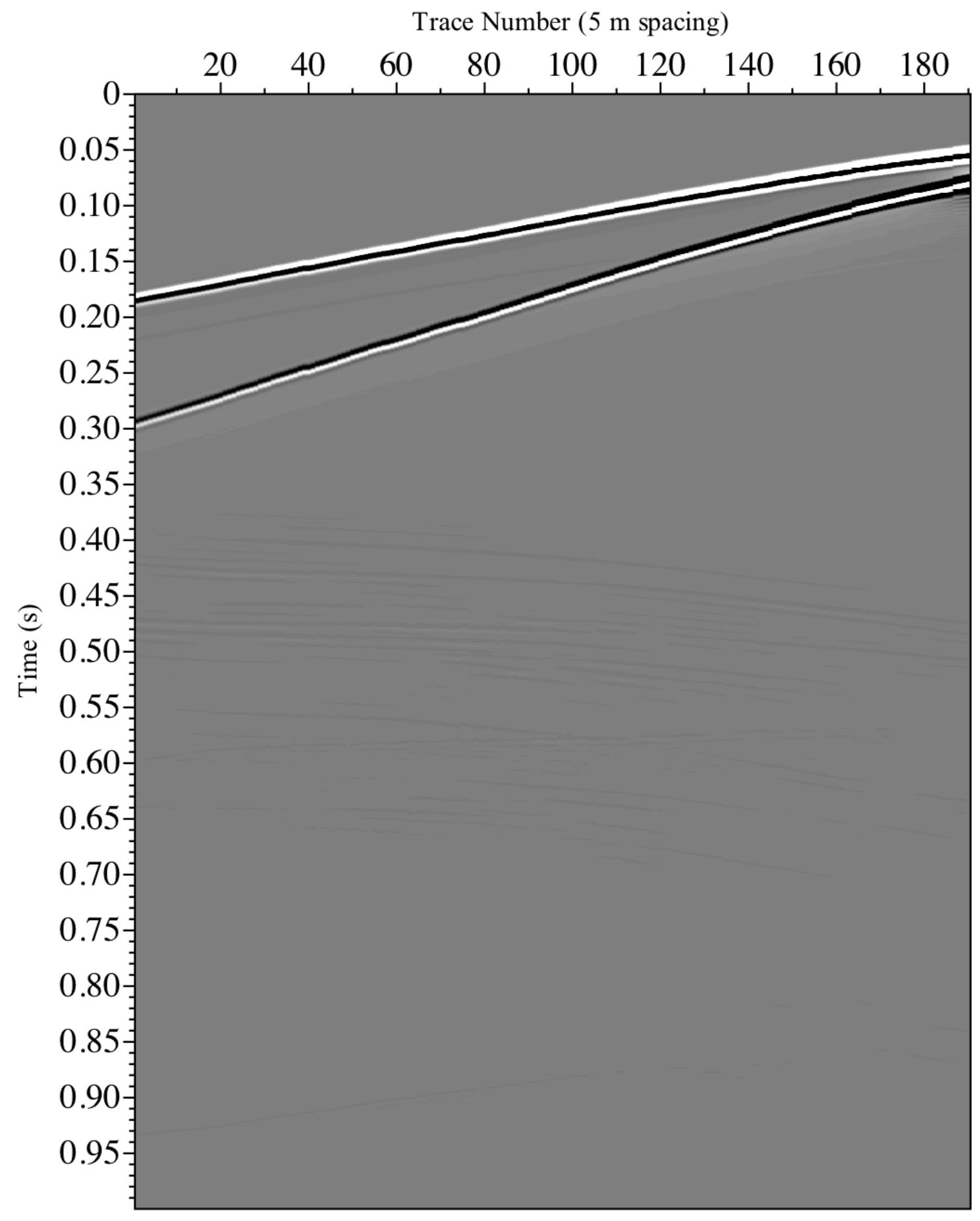

Appendix C.29 - Zero-offset northing particle velocity synthetic VSP data from 4Q66W3 in the 'Binary (777)' model. 


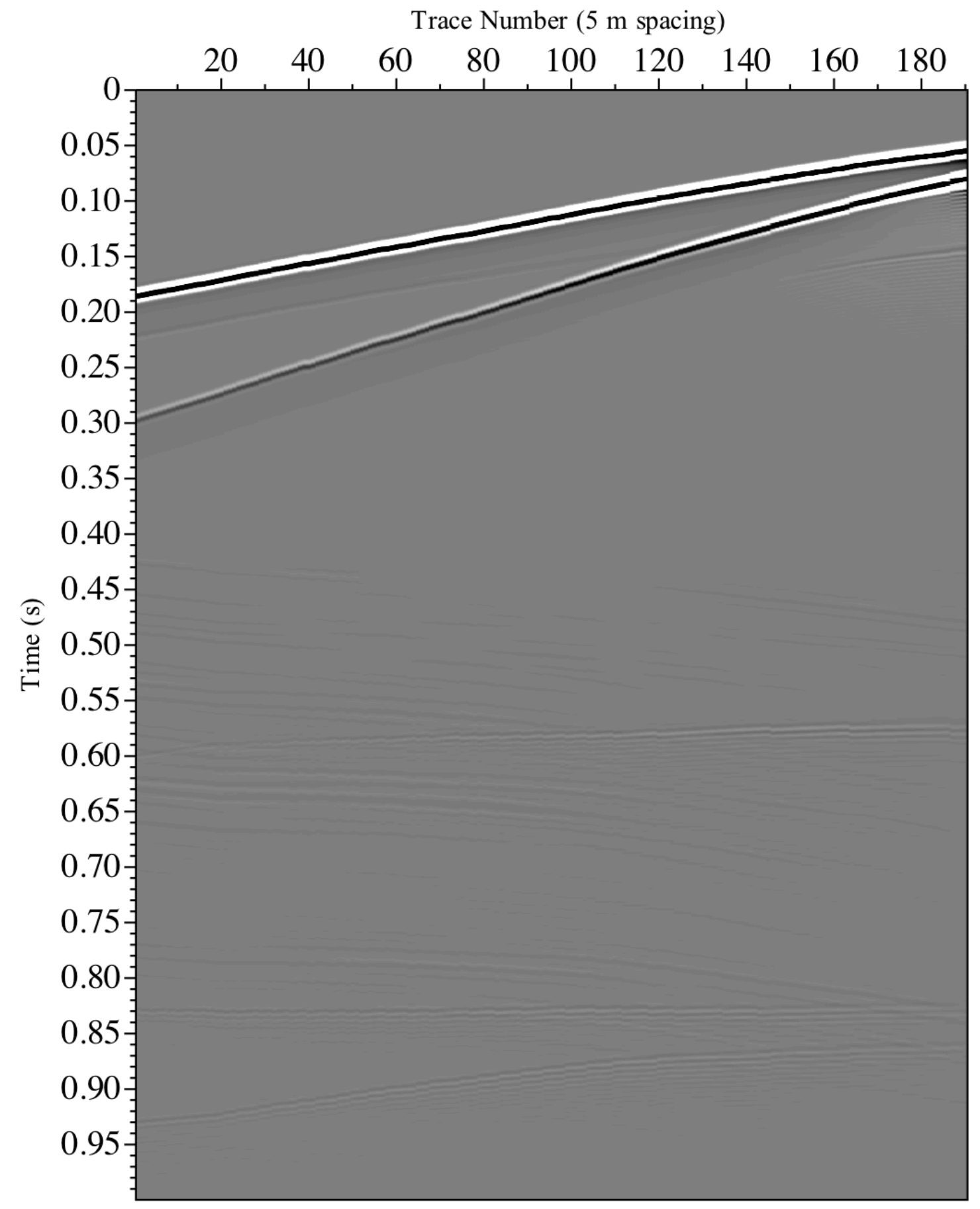

Appendix C.30 - Zero-offset vertical particle velocity synthetic VSP data from 4Q66W3 in the 'Binary (777)' model. 


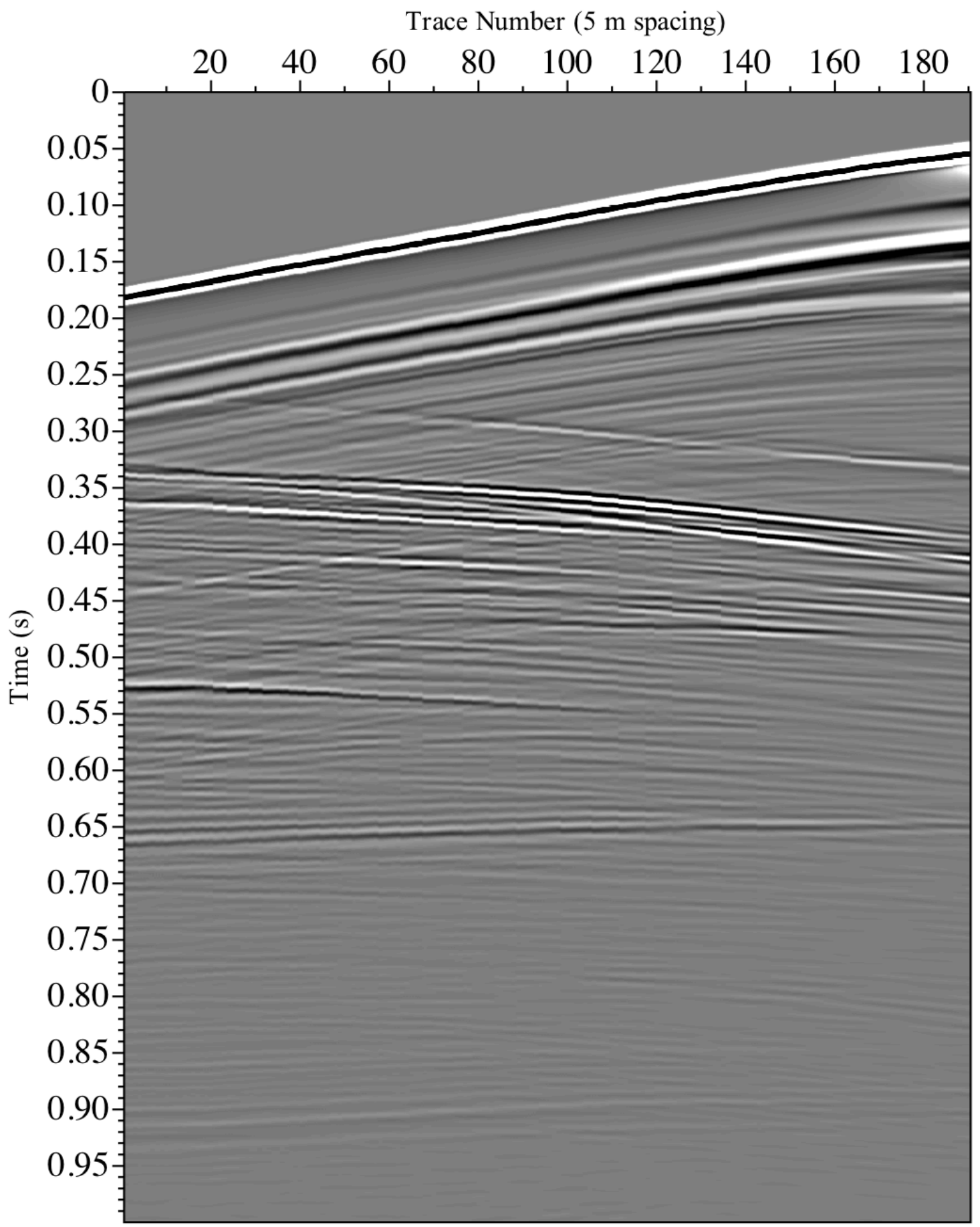

Appendix C.31 - Zero-offset divergence synthetic VSP data from 4Q66W3 in the 'Ore/

Missi’ model. 


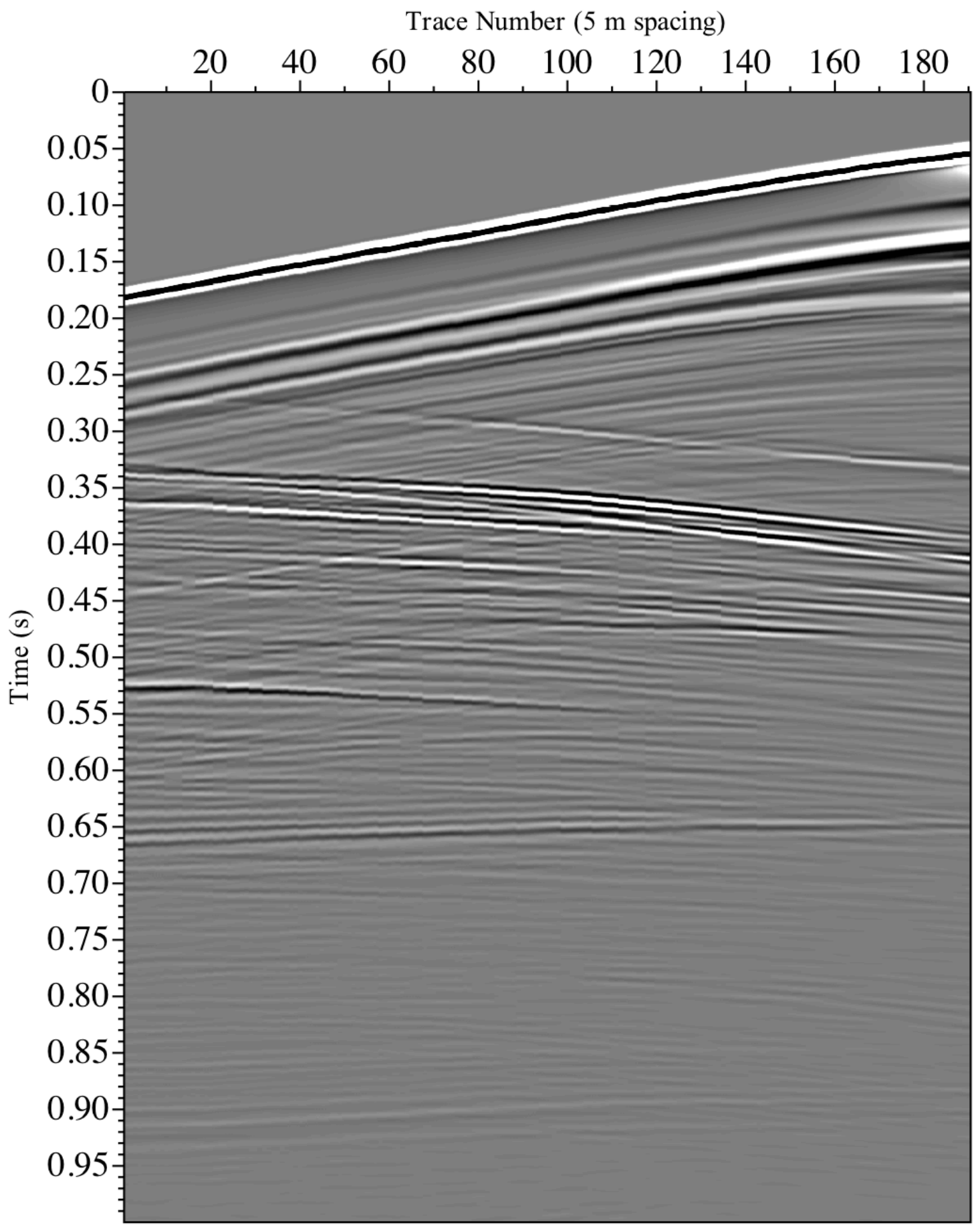

Appendix C.32 - Zero-offset pressure synthetic VSP data from 4Q66W3 in the 'Ore/

Missi' model. 


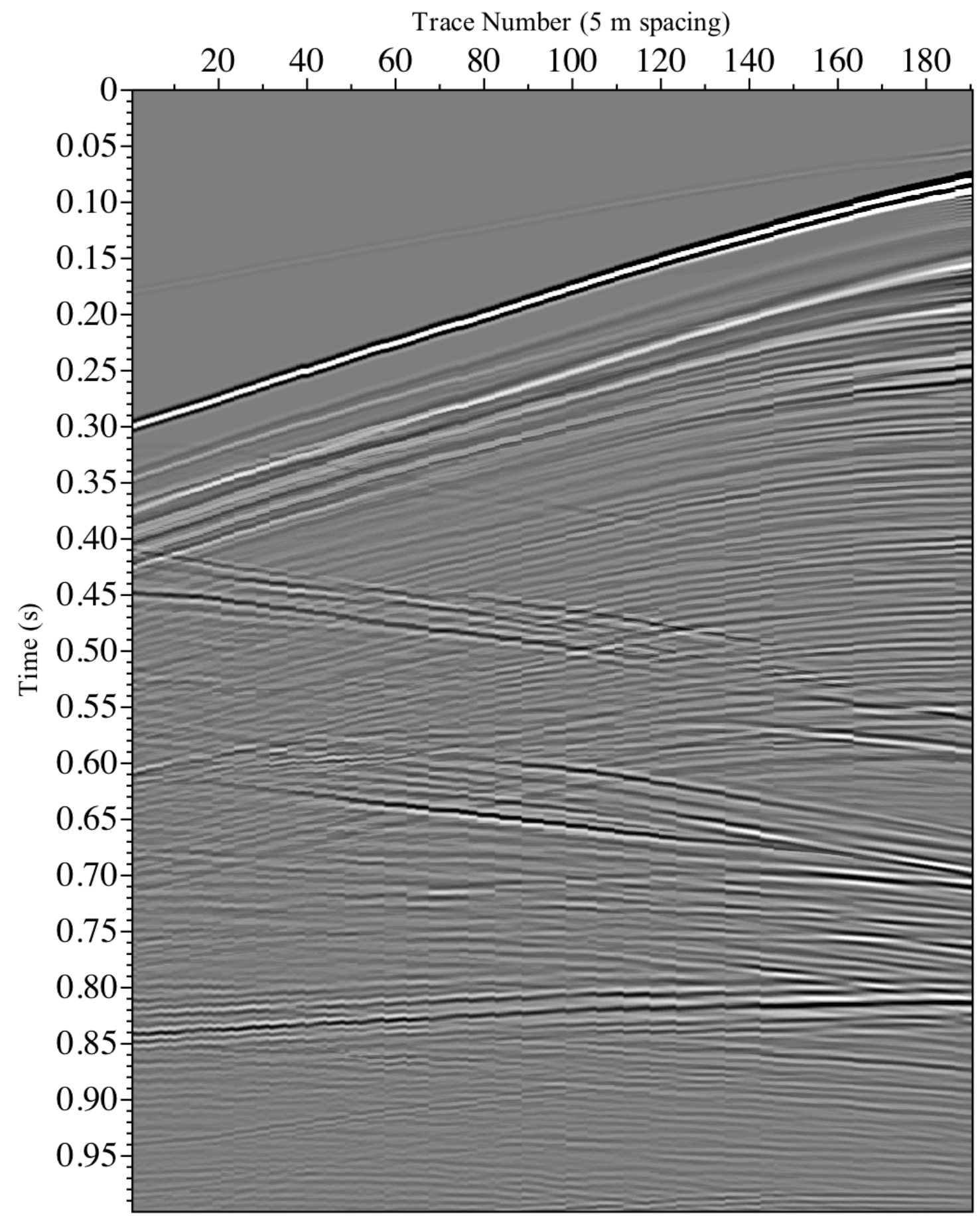

Appendix C.33 - Zero-offset rotation synthetic VSP data from 4Q66W3 in the 'Ore/

Missi' model. 


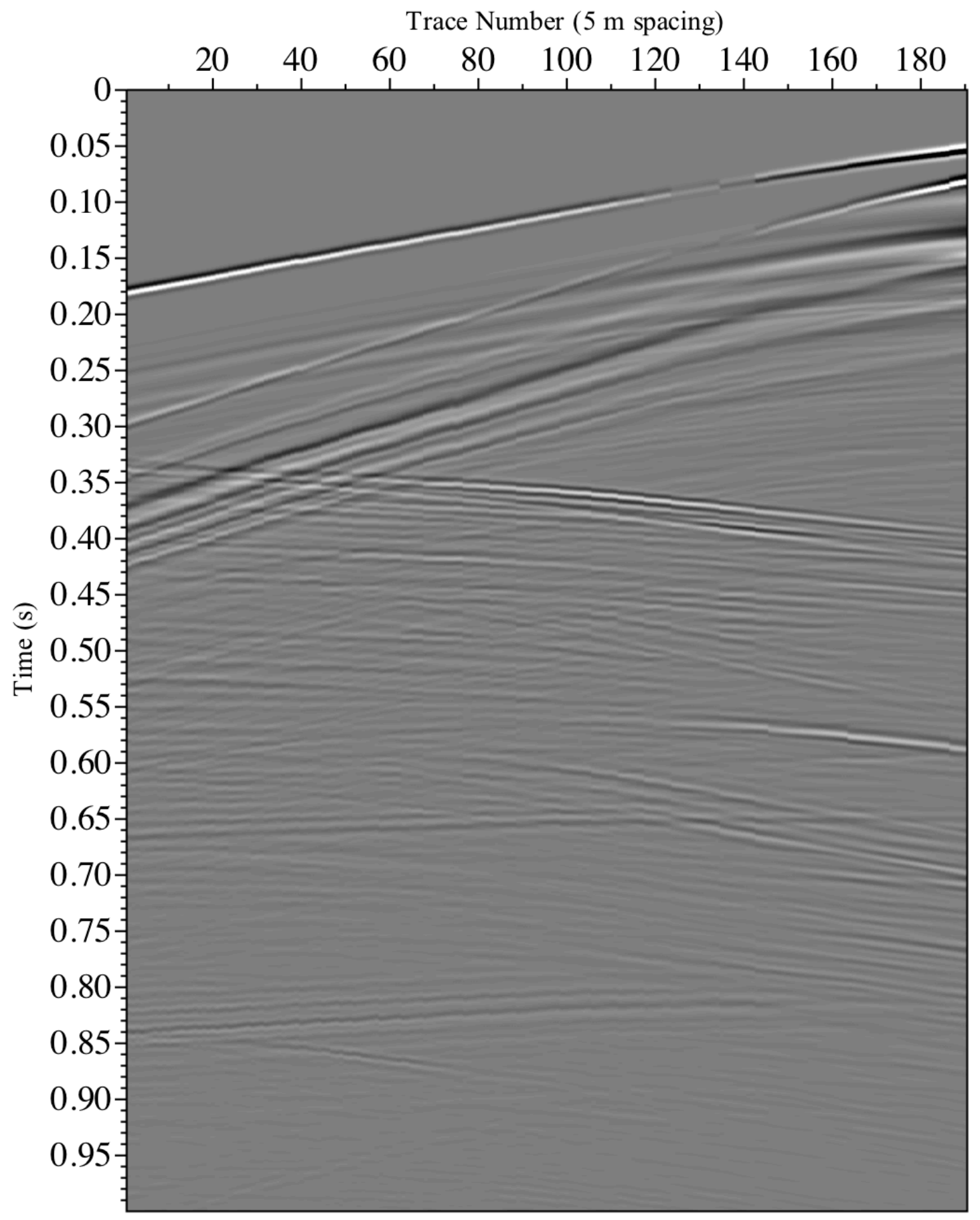

Appendix C.34 - Zero-offset easting particle velocity synthetic VSP data from 4Q66W3 in the 'Ore/Missi' model. 


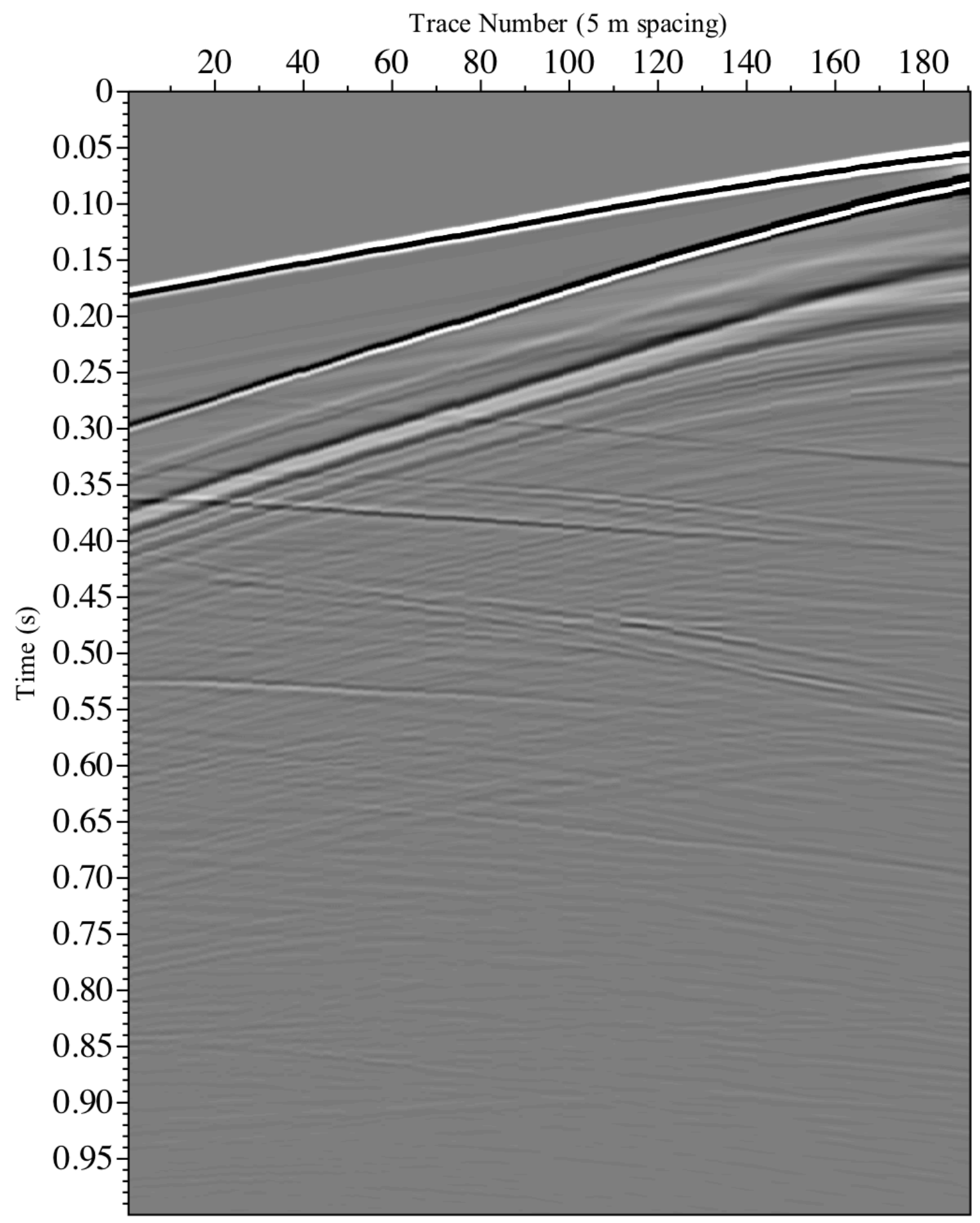

Appendix C.35 - Zero-offset northing particle velocity synthetic VSP data from 4Q66W3 in the 'Ore/Missi' model. 


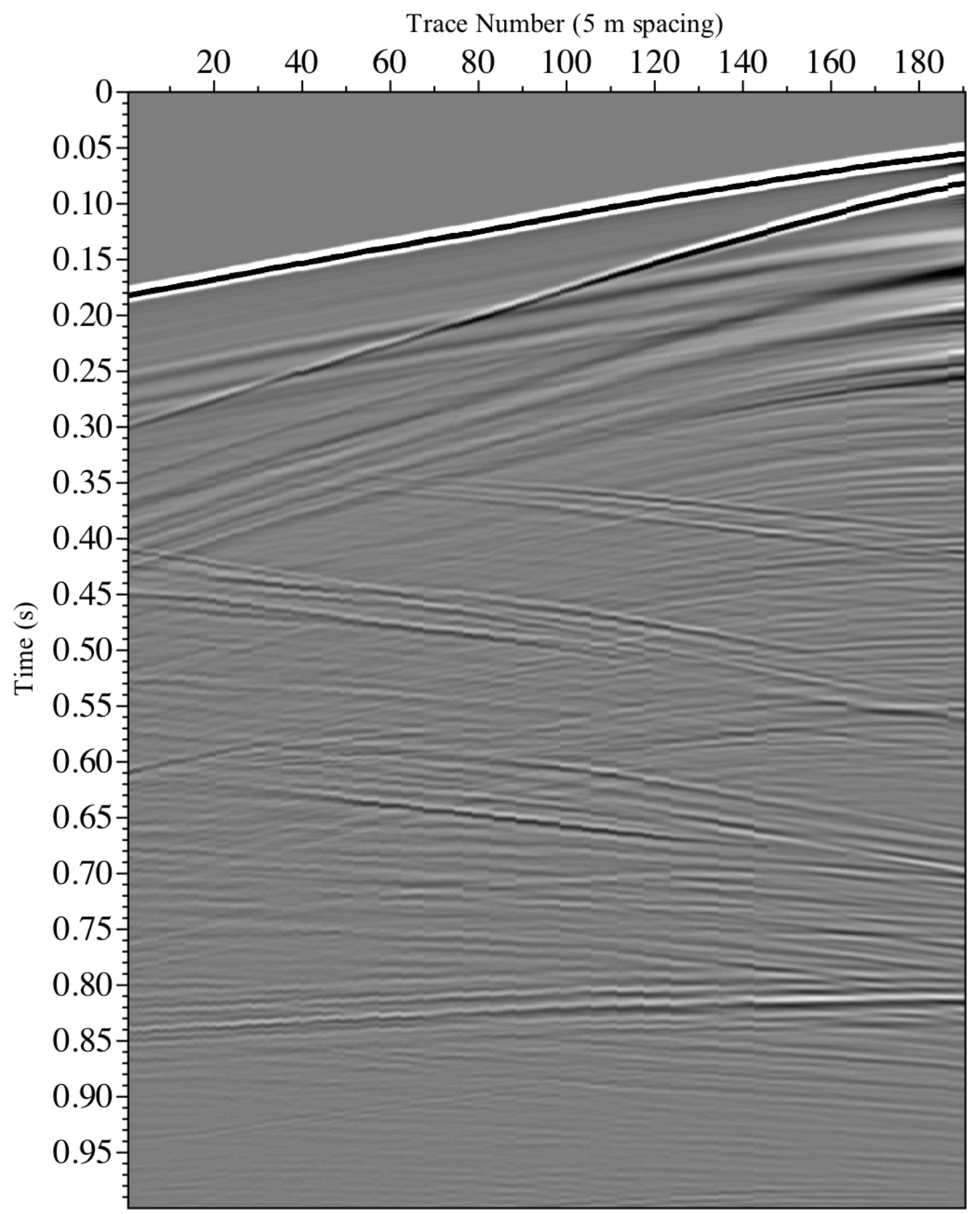

Appendix C.36 - Zero-offset vertical particle velocity synthetic VSP data from 4Q66W3 in the 'Ore/Missi' model. 


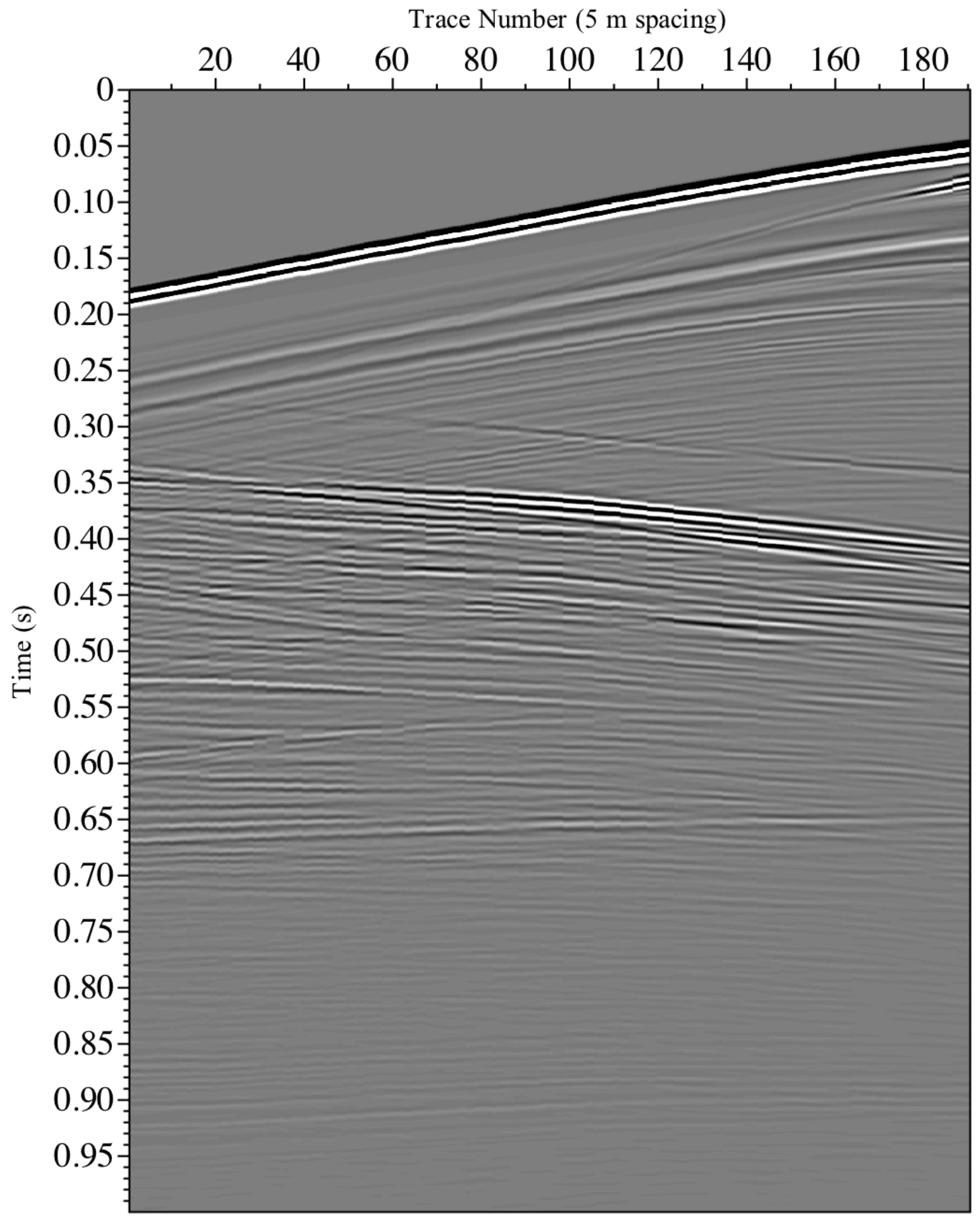

Appendix C.37 - Zero-offset divergence synthetic VSP data from 4Q66W3 in the 'Lithological' model. 


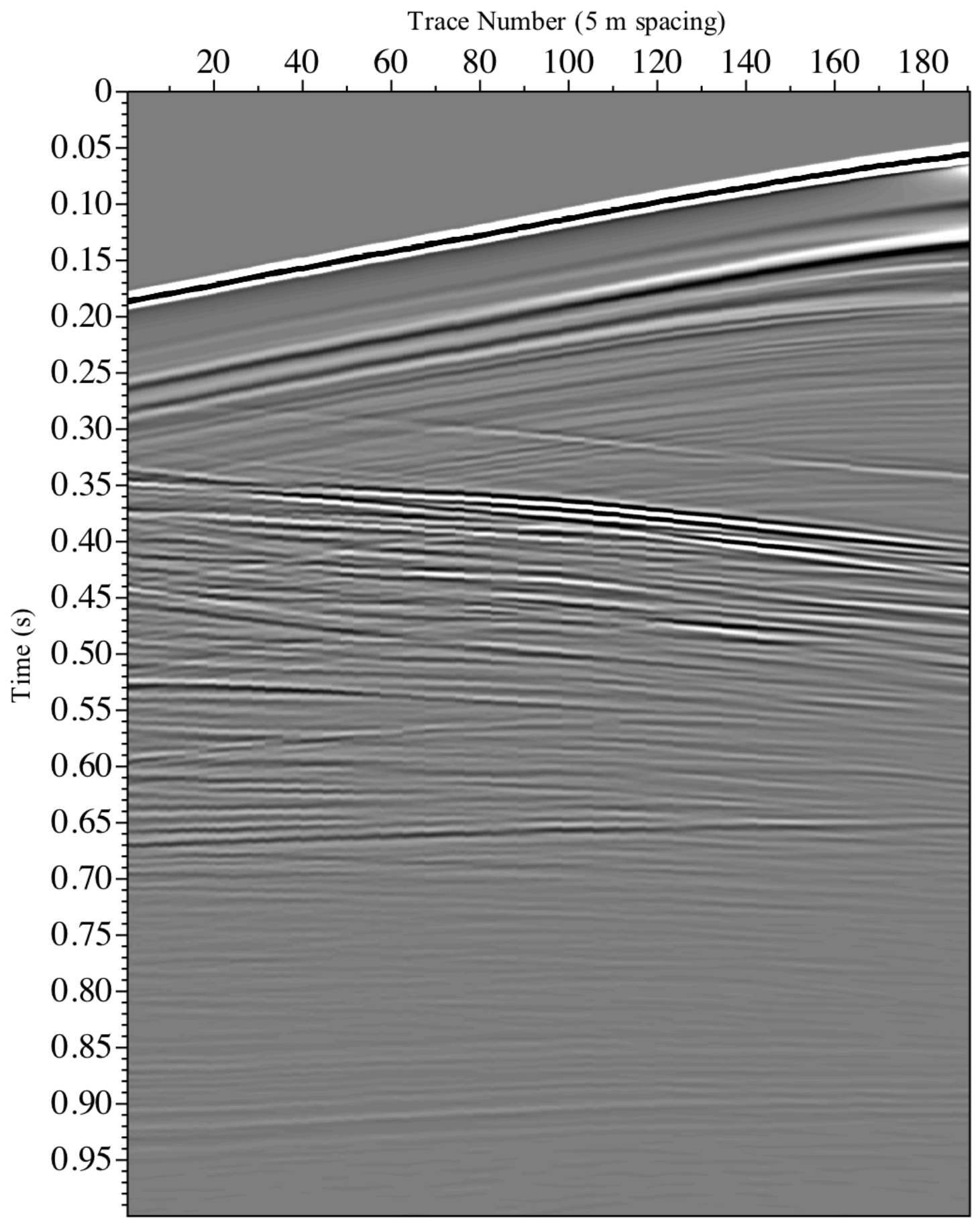

Appendix C.38 - Zero-offset pressure synthetic VSP data from 4Q66W3 in the

'Lithological' model. 


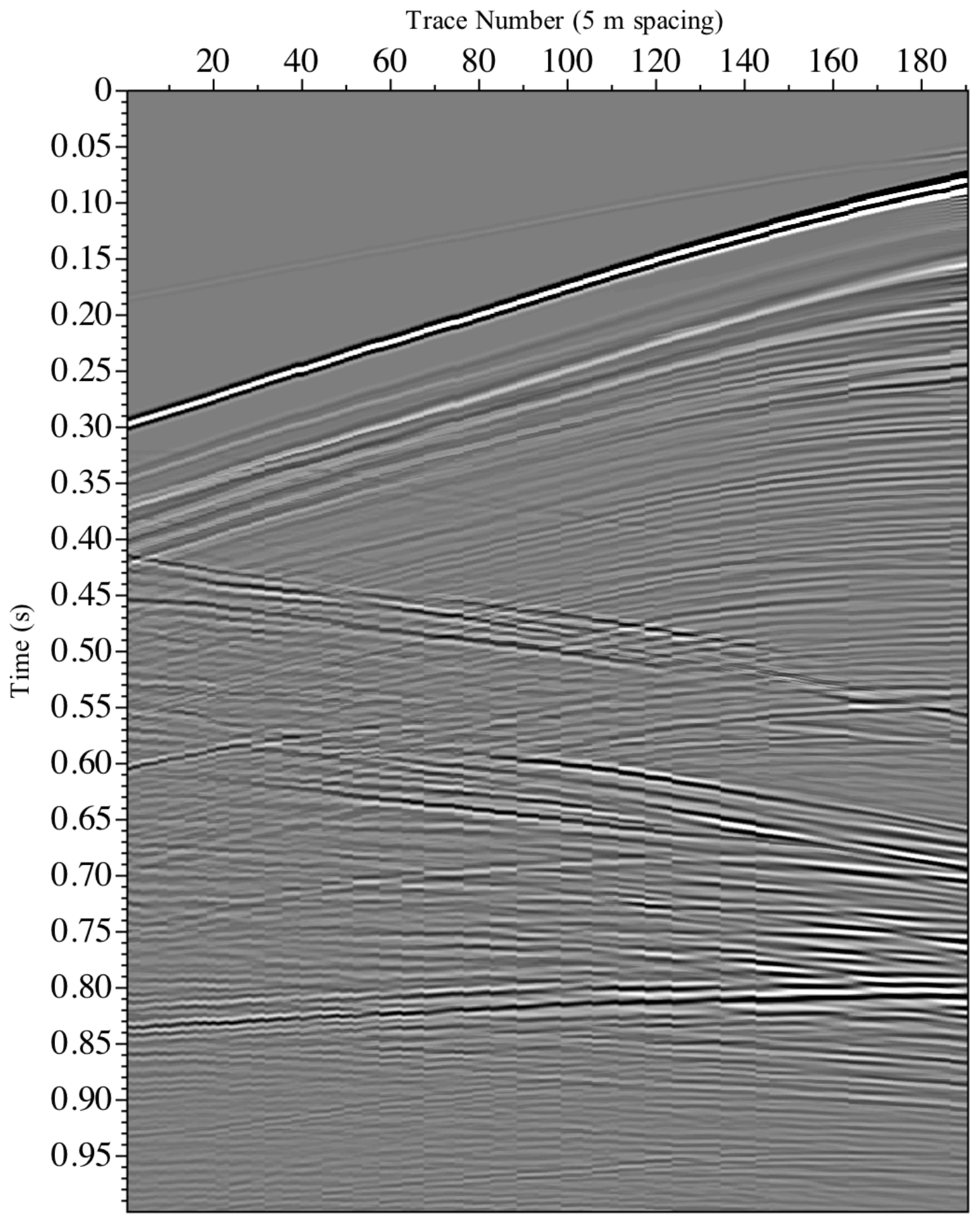

Appendix C.39 - Zero-offset rotation synthetic VSP data from 4Q66W3 in the

'Lithological' model. 


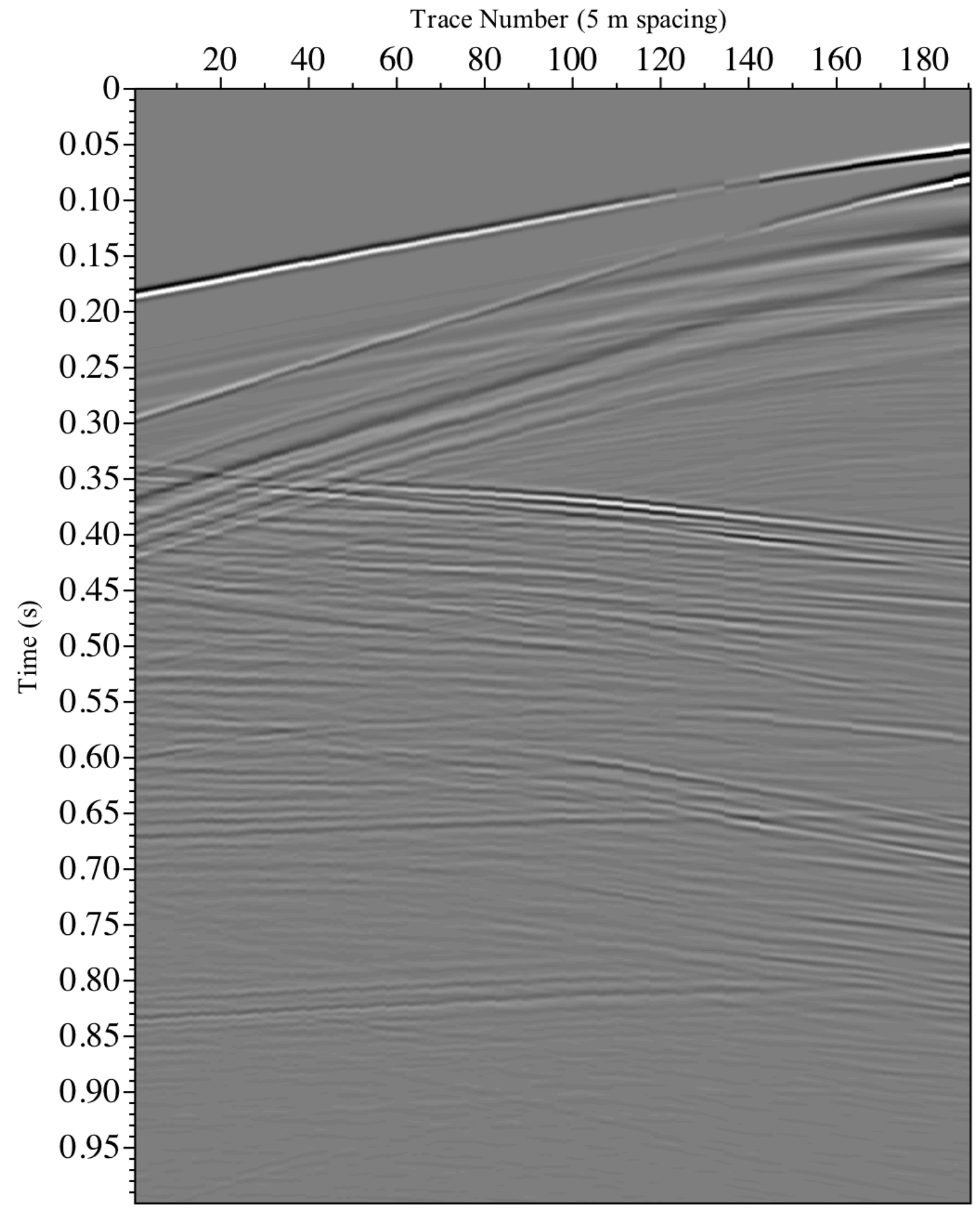

Appendix C.40 - Zero-offset easting particle velocity synthetic VSP data from 4Q66W3 in the 'Lithological' model. 


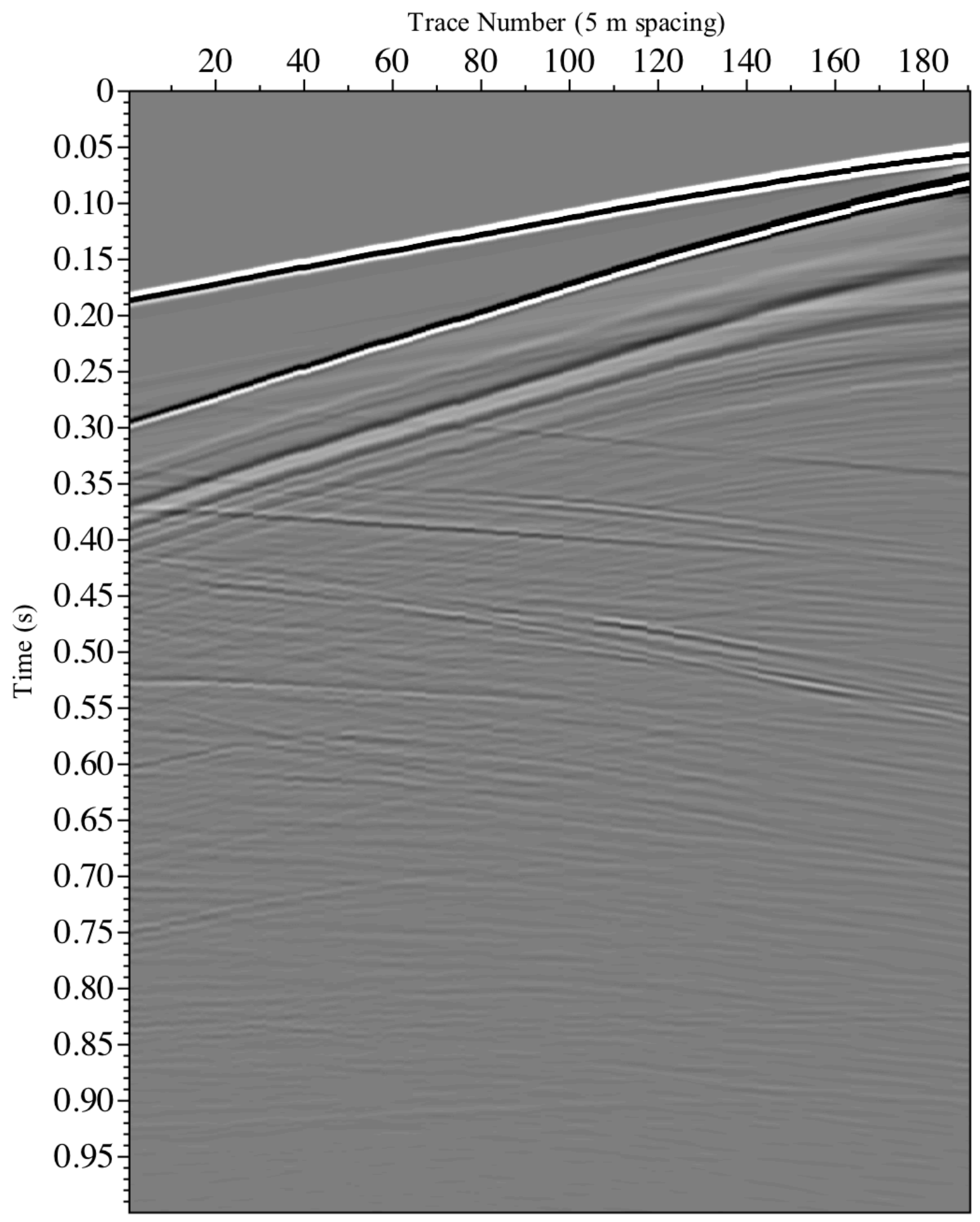

Appendix C.41 - Zero-offset northing particle velocity synthetic VSP data from 4Q66W3 in the 'Lithological' model. 


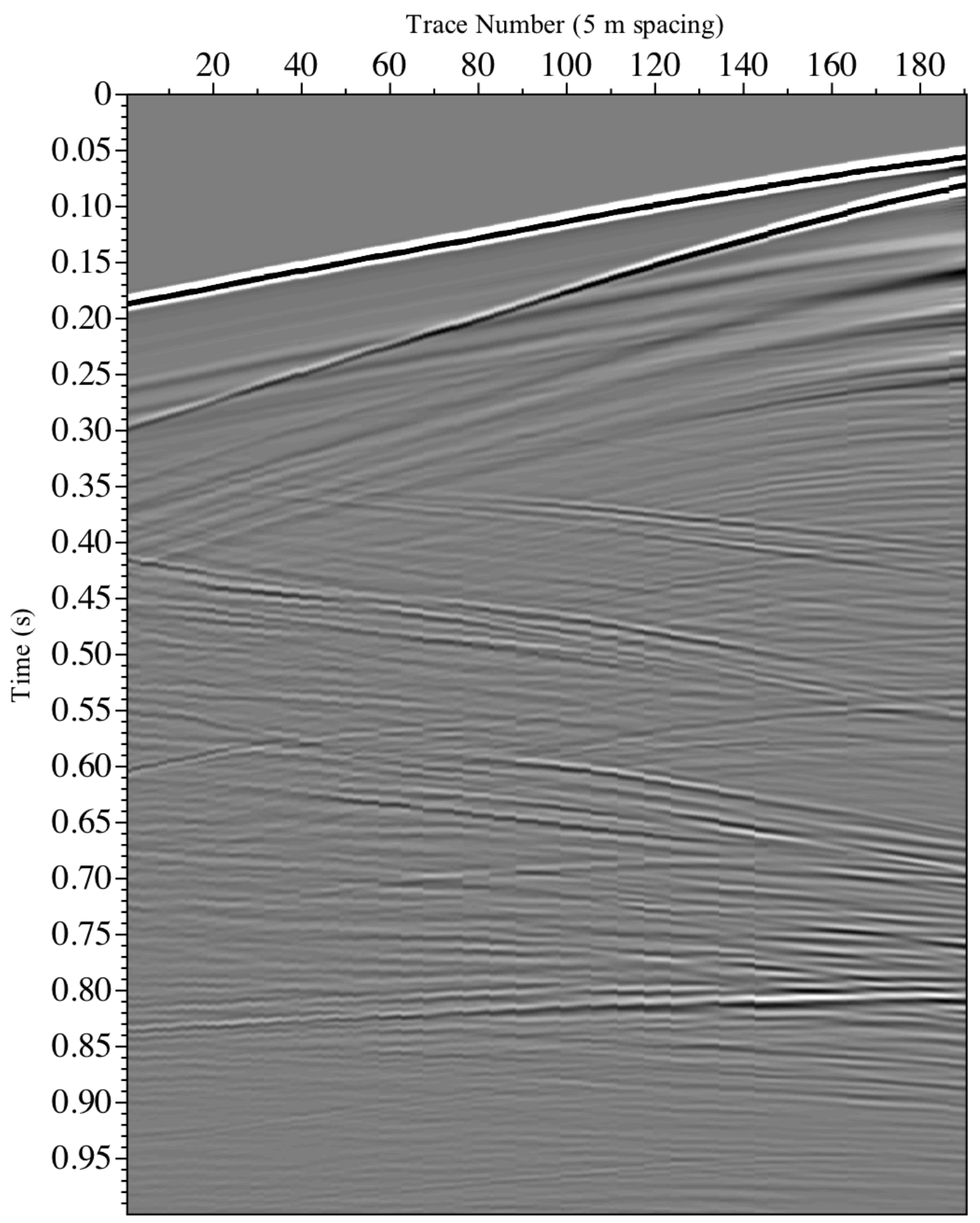

Appendix C.42 - Zero-offset vertical particle velocity synthetic VSP data from 4Q66W3 in the 'Lithological' model. 


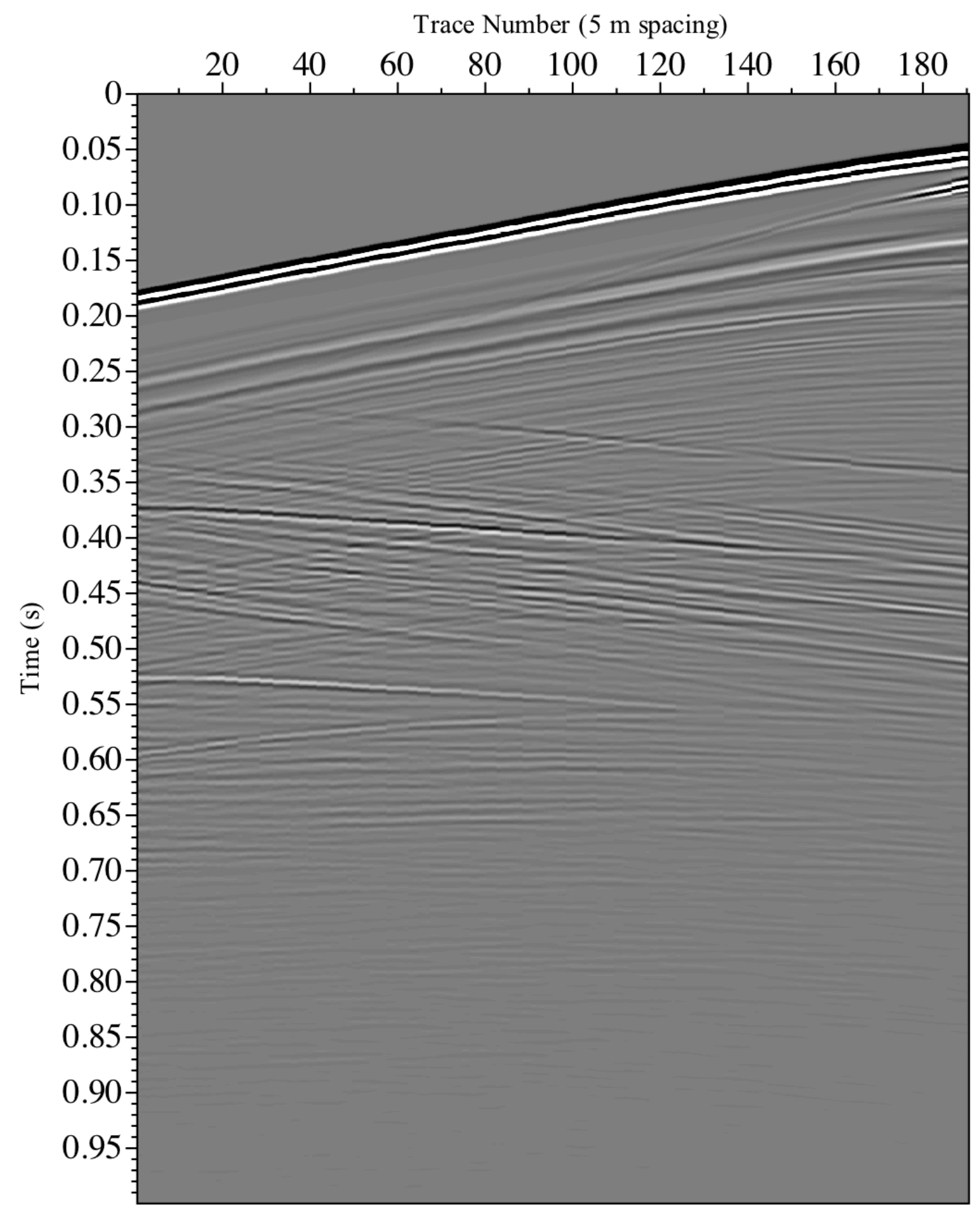

Appendix C.43 - Zero-offset divergence synthetic VSP data from 4Q66W3 in the "No

Ore' model. 


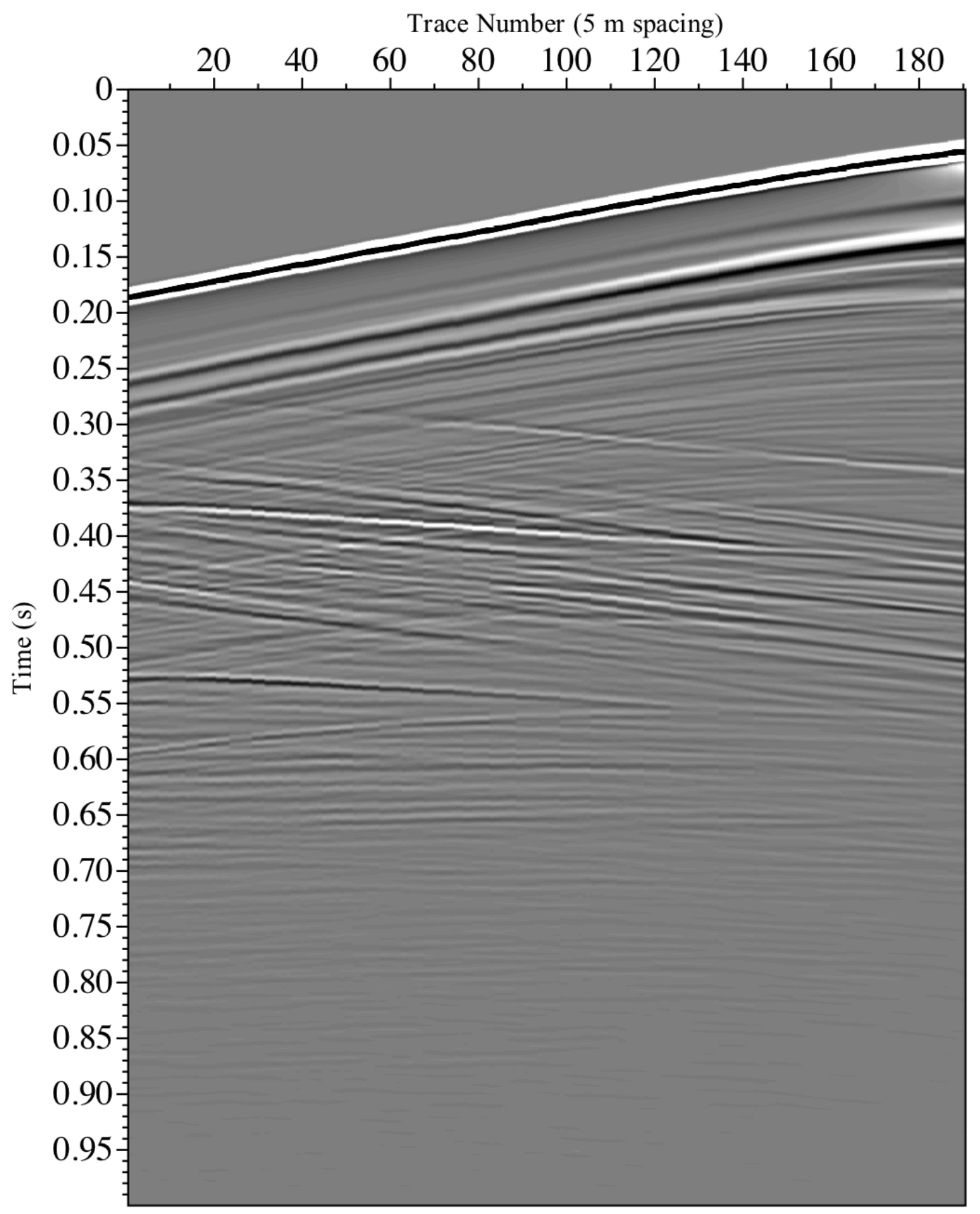

Appendix C.44 - Zero-offset pressure synthetic VSP data from 4Q66W3 in the 'No Ore' model. 


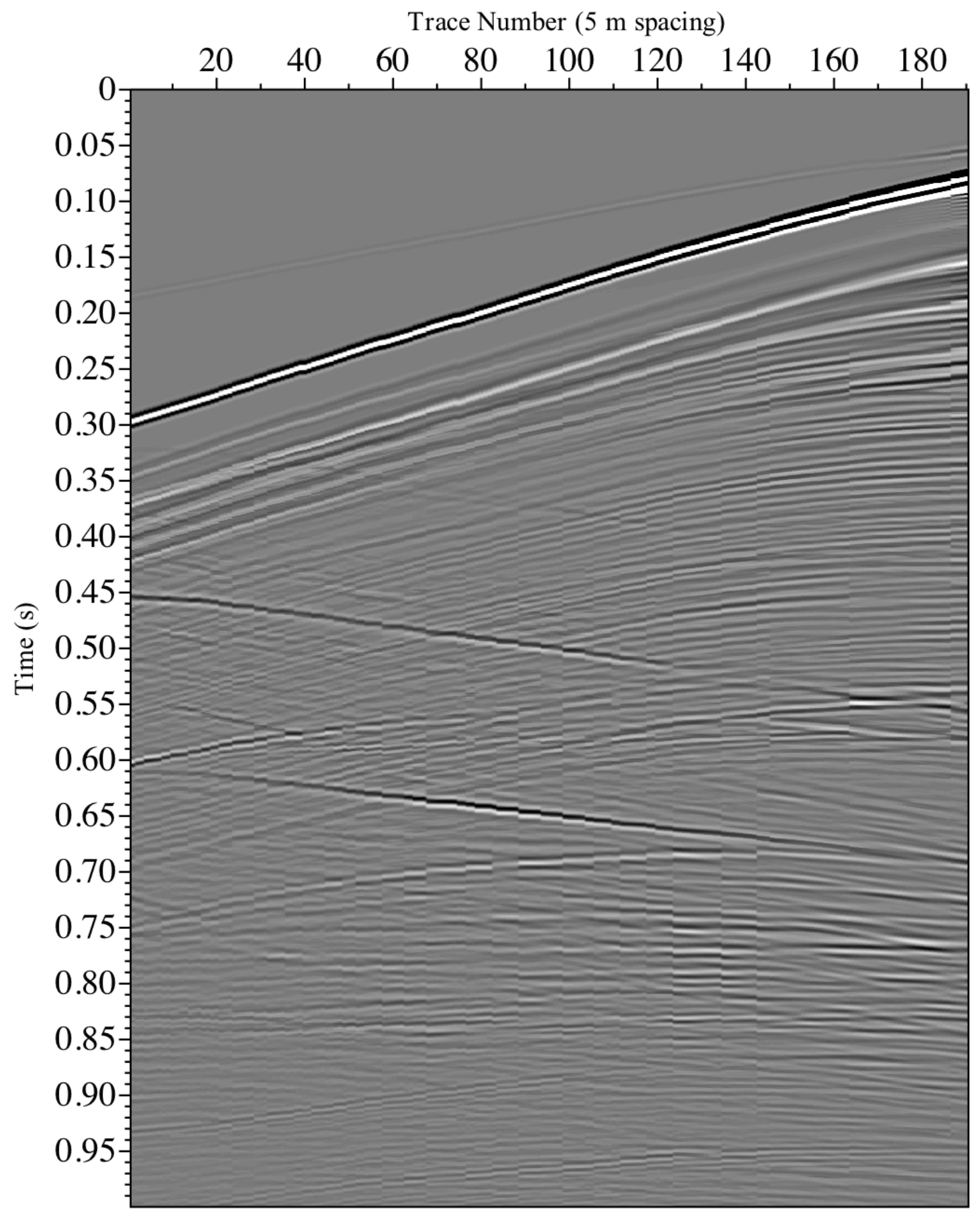

Appendix C.45 - Zero-offset rotation synthetic VSP data from 4Q66W3 in the 'No Ore' model. 


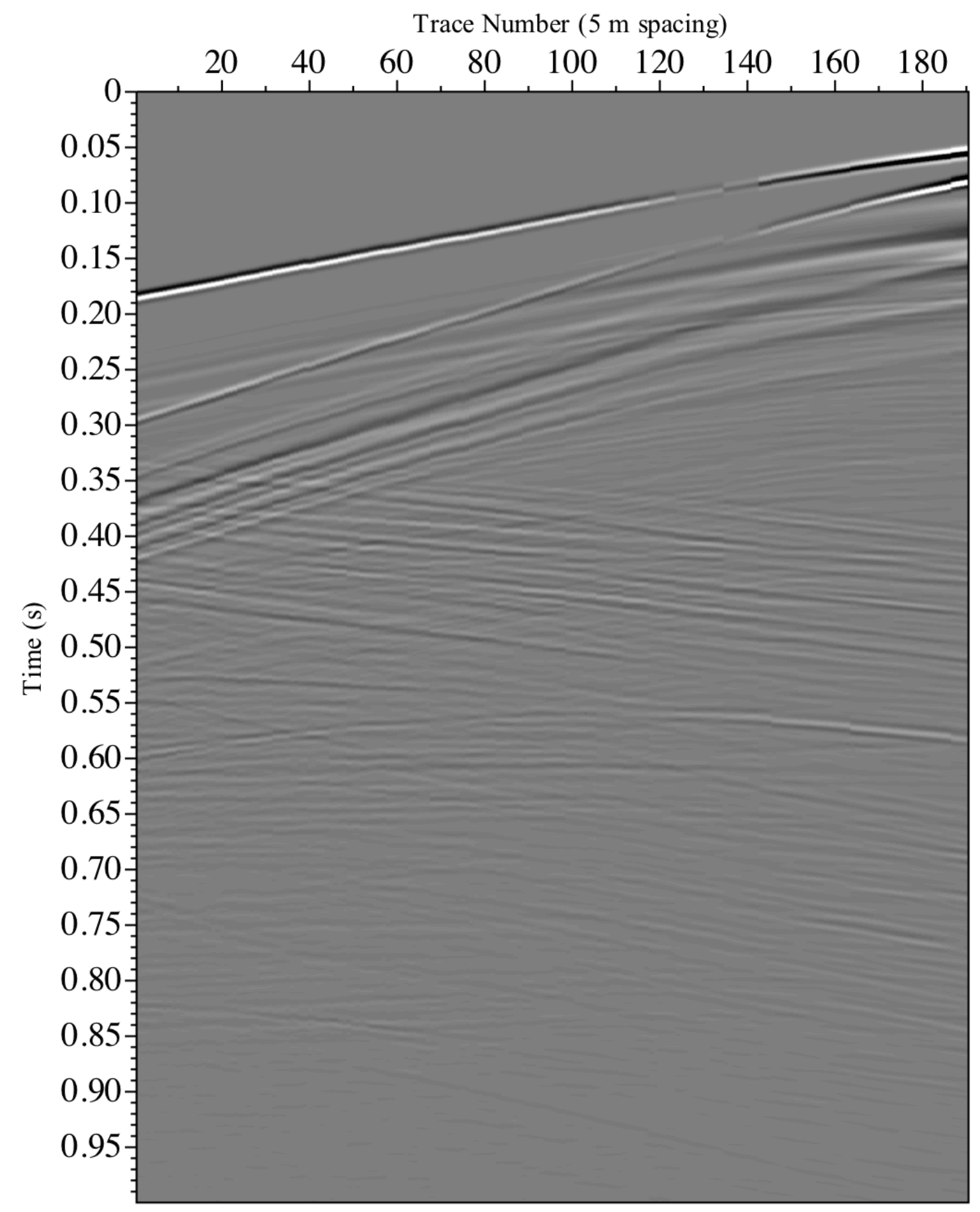

Appendix C.46 - Zero-offset easting particle velocity synthetic VSP data from 4Q66W3 in the 'No Ore' model. 


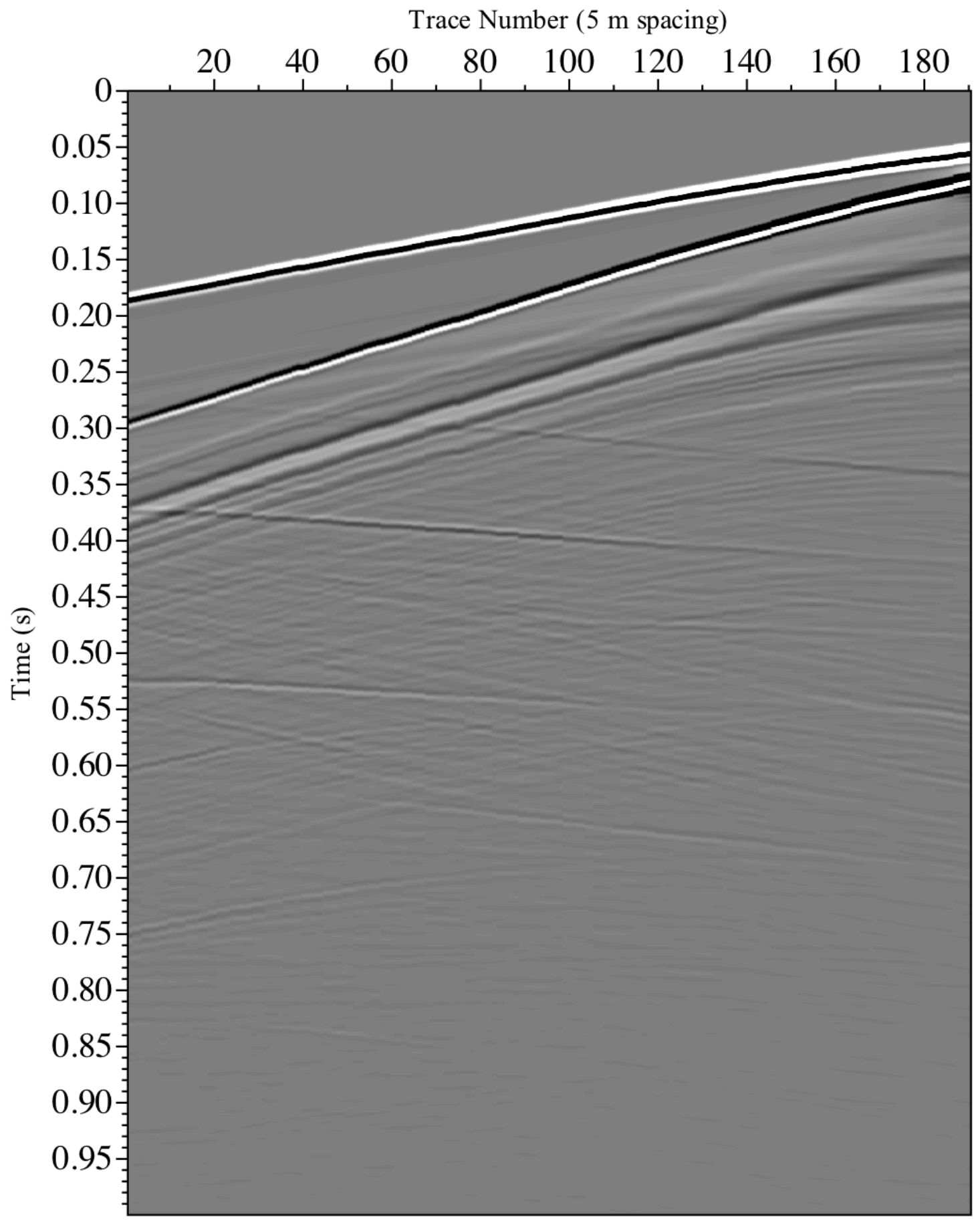

Appendix C.47 - Zero-offset northing particle velocity synthetic VSP data from 4Q66W3 in the 'No Ore' model. 


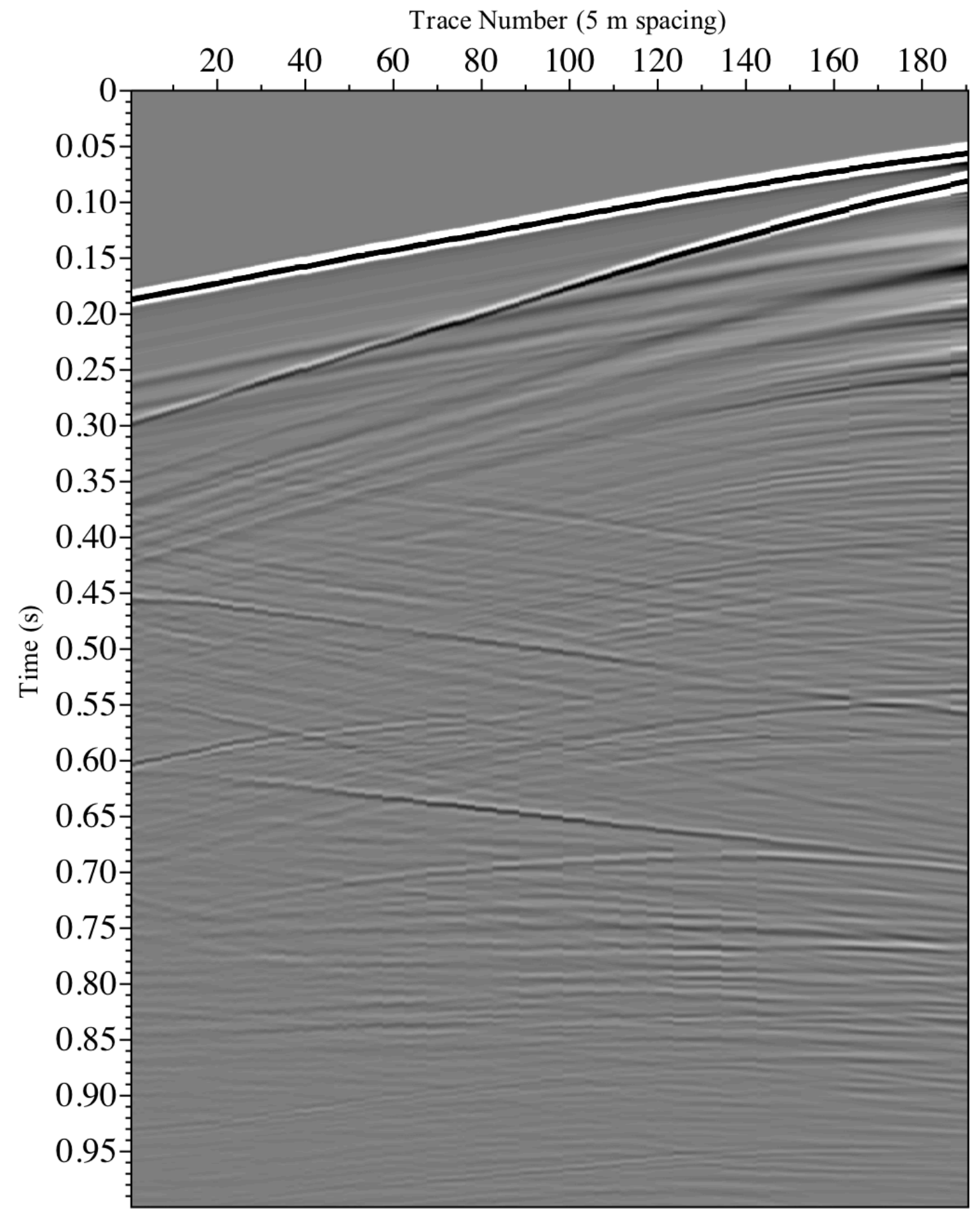

Appendix C.48 - Zero-offset vertical particle velocity synthetic VSP data from 4Q66W3 in the 'No Ore' model. 


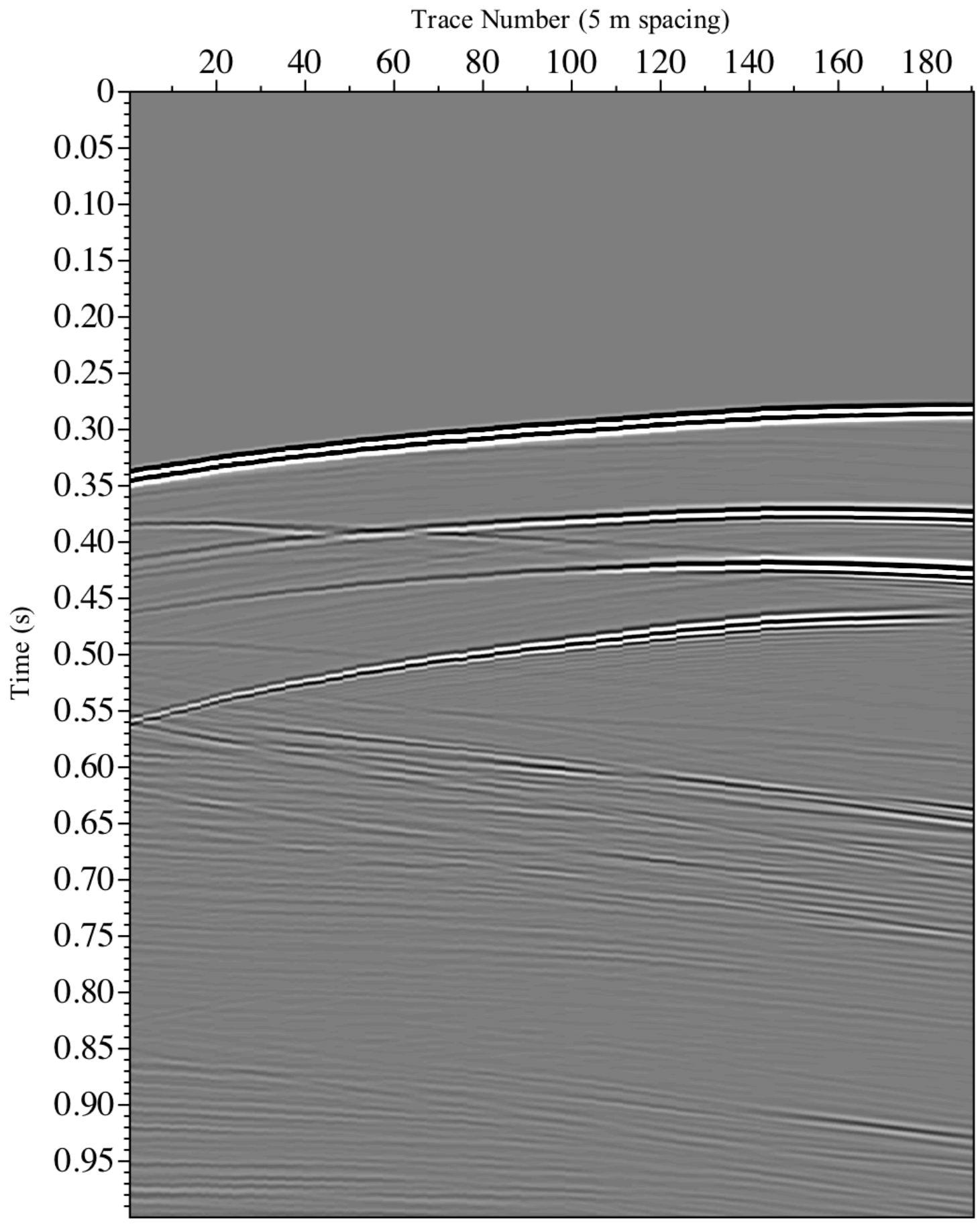

Appendix C.49 - Far-offset divergence synthetic VSP data from 4Q66W3 in the

'Lithological' model. 


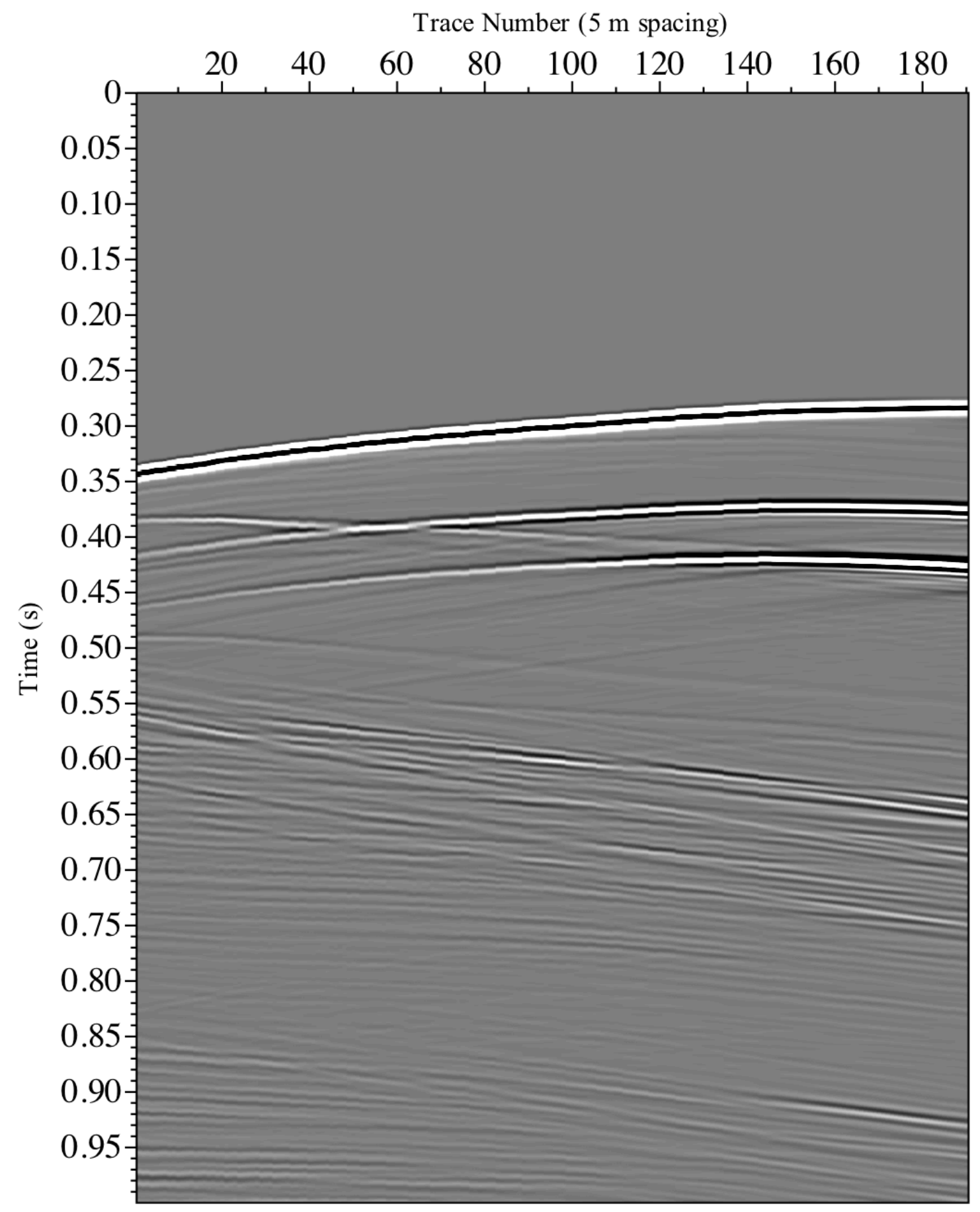

Appendix C.50 - Far-offset pressure synthetic VSP data from 4Q66W3 in the

'Lithological' model. 


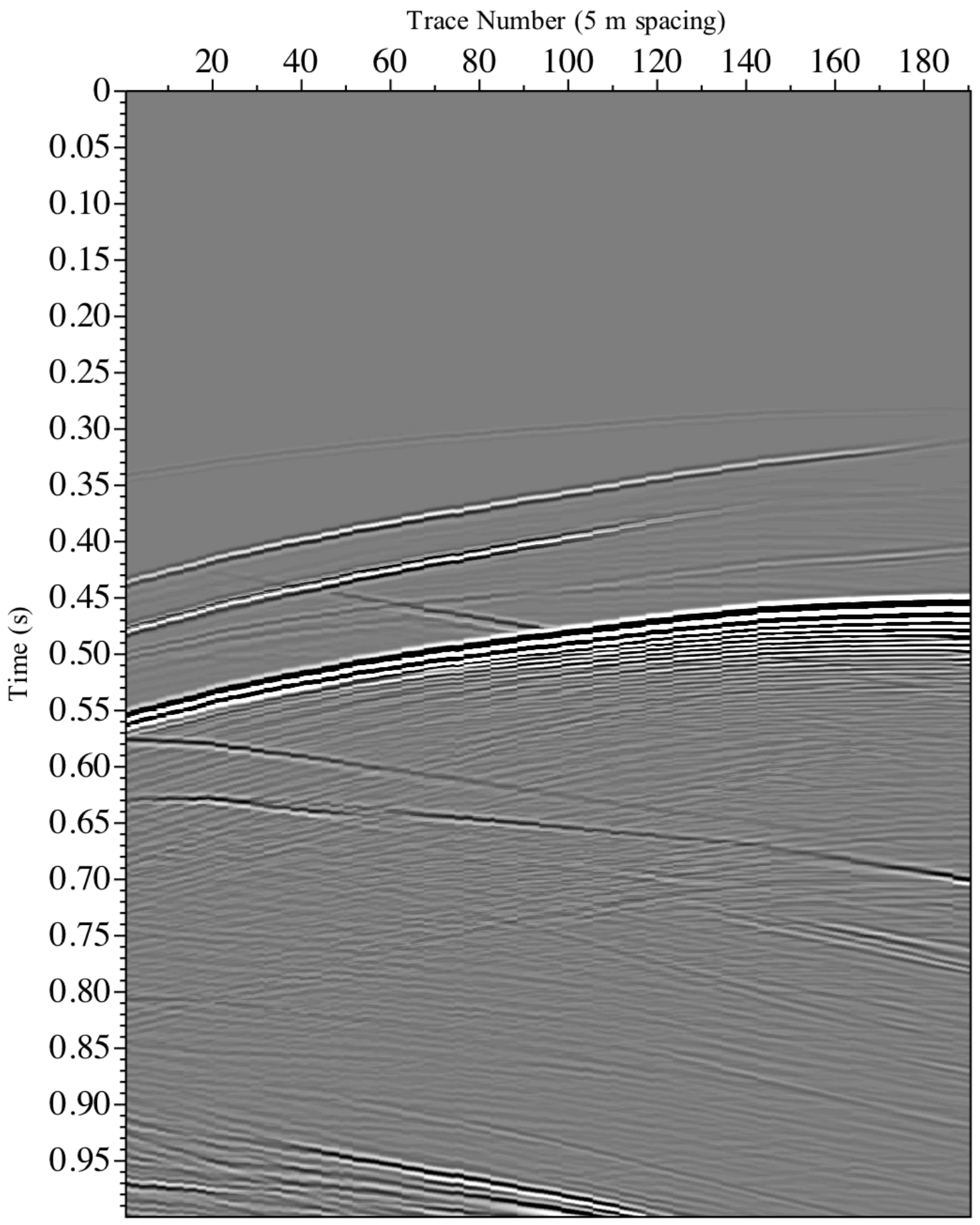

Appendix C.51 - Far-offset rotation synthetic VSP data from 4Q66W3 in the

'Lithological' model. 


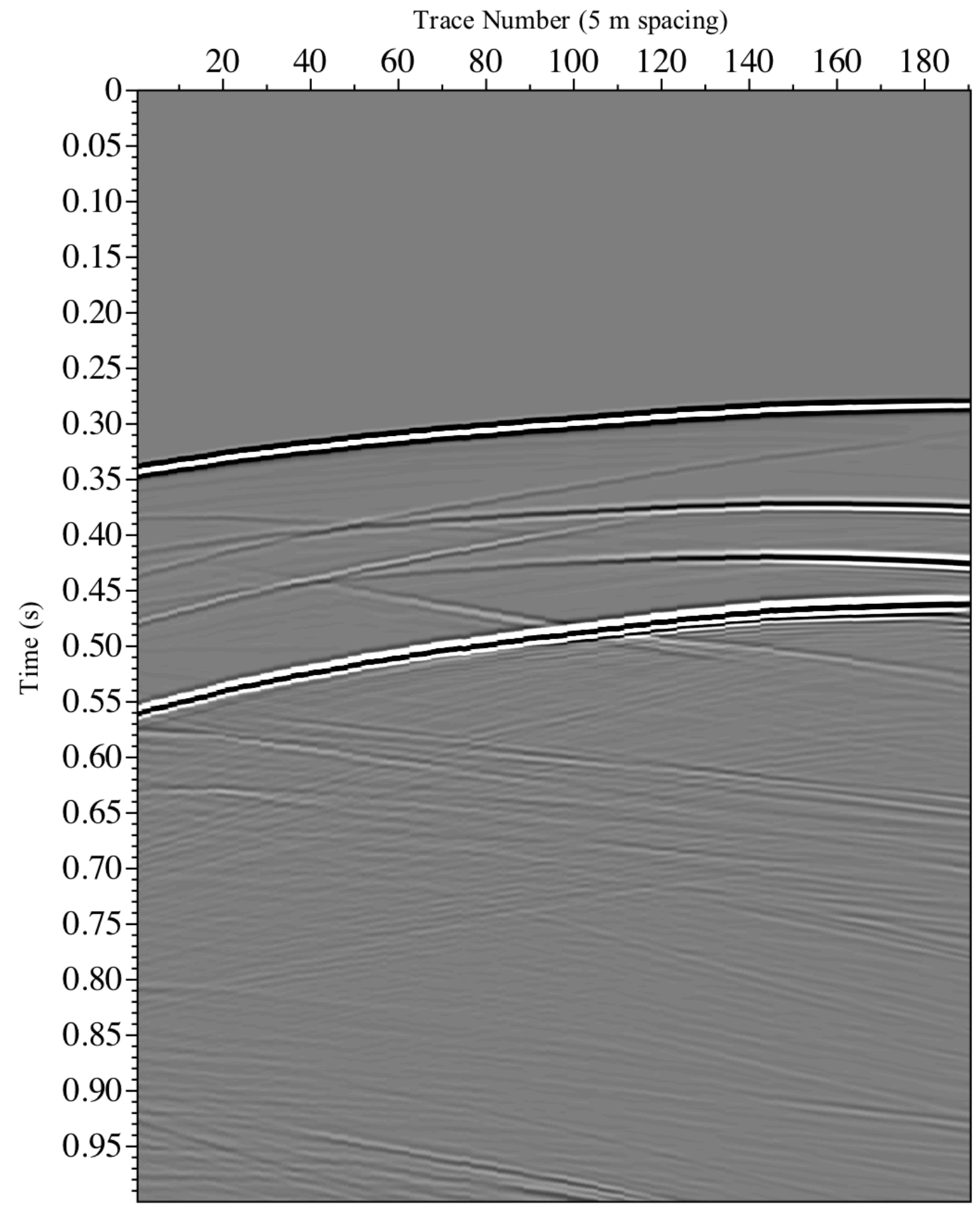

Appendix C.52 - Far-offset easting particle velocity synthetic VSP data from 4Q66W3 in the 'Lithological' model. 


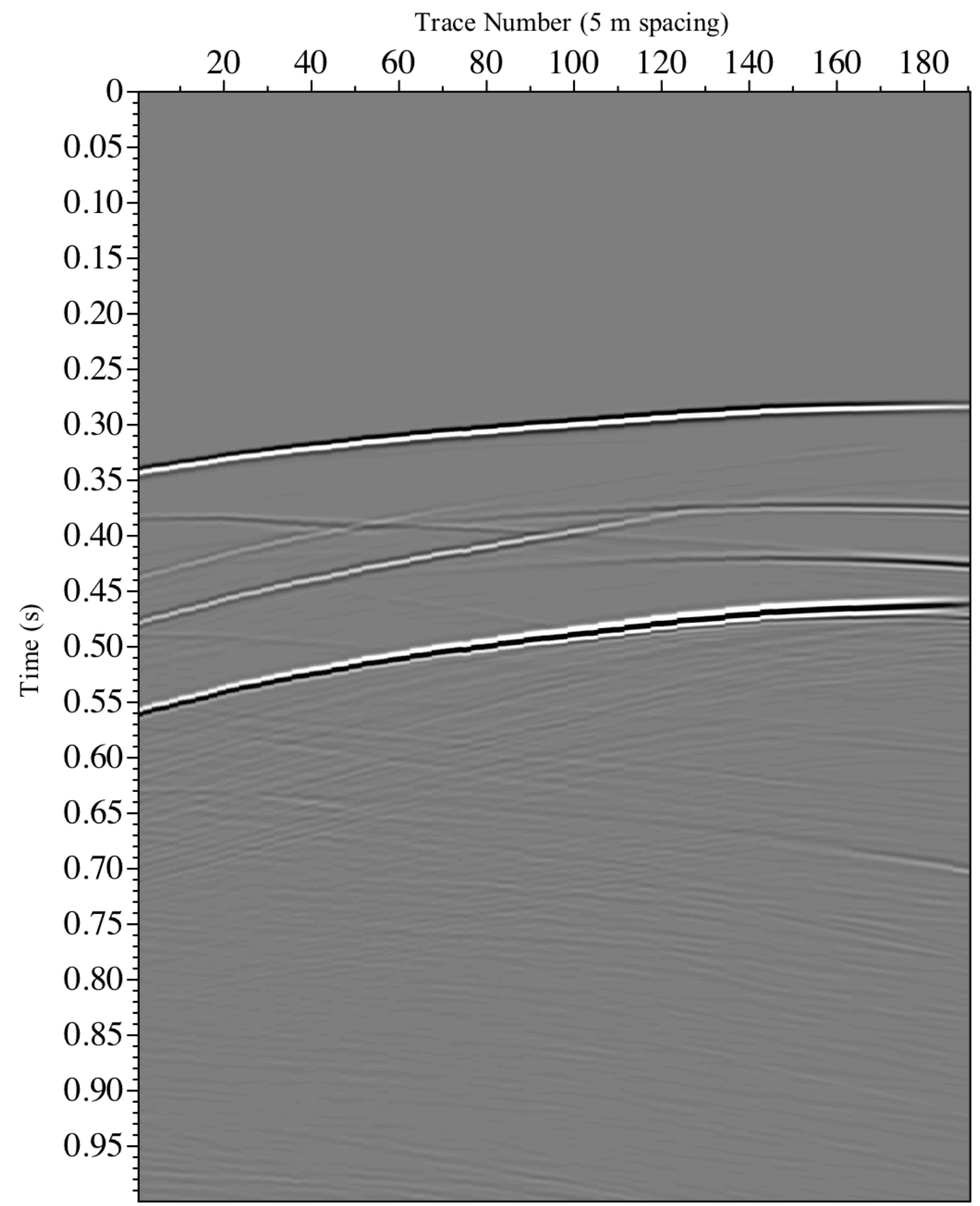

Appendix C.53 - Far-offset northing particle velocity synthetic VSP data from 4Q66W3 in the 'Lithological' model. 


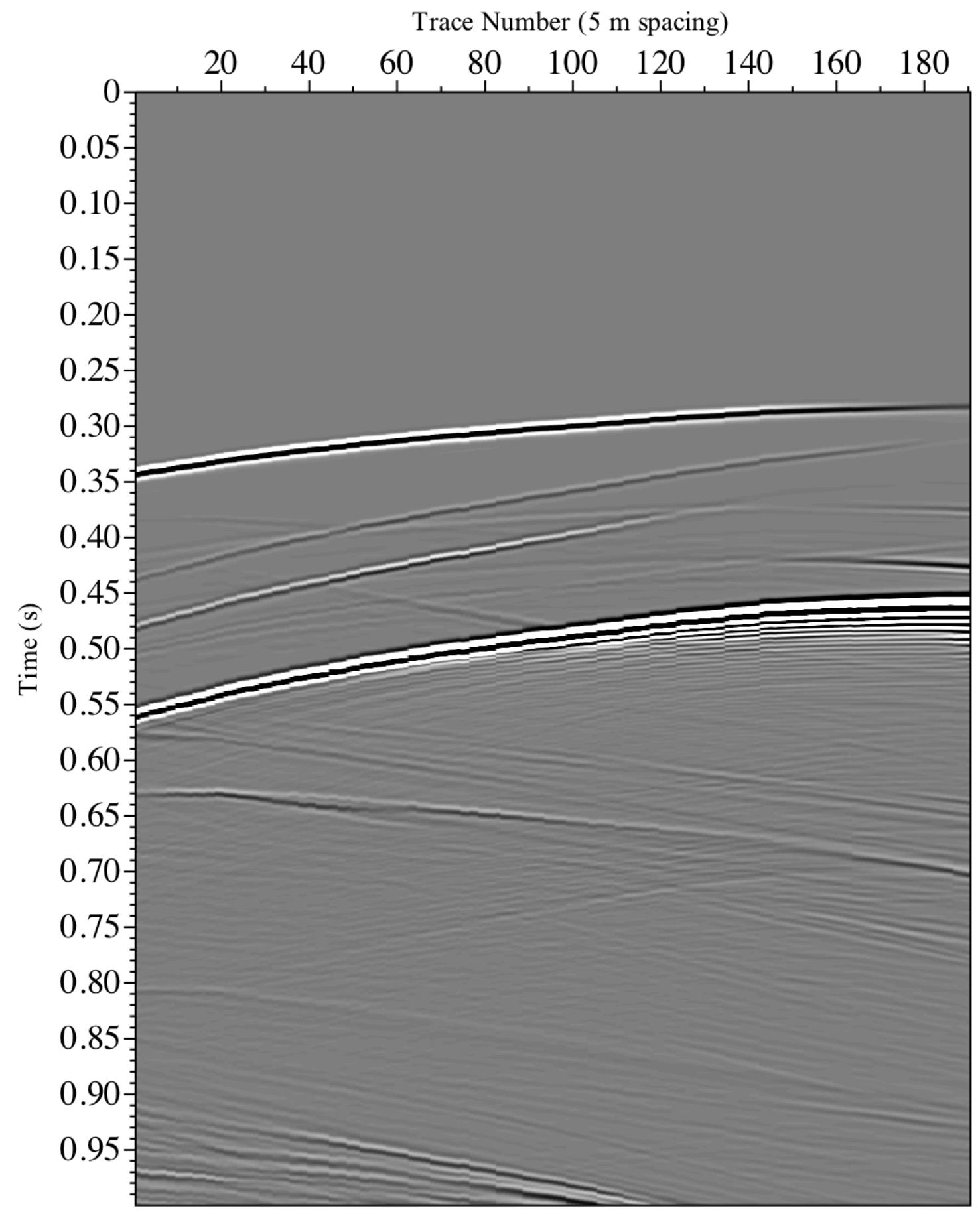

Appendix C.54 - Far-offset vertical particle velocity synthetic VSP data from 4Q66W3 in the 'Lithological' model. 


\section{Appendix D - Abstract: CGU Annual Scientific Meeting 2013, Saskatoon}

\section{TARGETING VMS ORE LENSES USING VERTICAL SEISMIC PROFILING, FLIN FLON MANITOBA. \\ D. Melanson ${ }^{1}$, D.J. White ${ }^{1,2}$, C. Samson 1 , G. Bellefleur ${ }^{2}$. ${ }^{1}$ Department of Earth Sciences, Carleton University, Ottawa, Ontario, Canada K1S 5B6 (dave_melanson@carleton.ca - MSc. project, claire_samson@carleton.ca), ${ }^{2}$ Natural Resources Canada, Ottawa, Ontario, Canada K1A 0E8 (Don.White@NRCan-RNCan.gc.ca, Gilles.Bellefleur@NRCan- RNCan.gc.ca)}

Between October 12th and 18th, 2006, VSP data were acquired from 3 boreholes in Flin Flon, Manitoba: 4Q66W3, FFM001 and FFS039, as part of the Targeted Geoscience Initiative (TGI-3). The Flin Flon belt is richly endowed with VMS deposits including the 85.5 Mt Flin Flon-Callinan-777 pyrrhotite and sphalerite-rich ore system. Surface 2D and 3D seismic data were also acquired as part of this project. VSP is a downhole seismic technique, whereby a string of geophones is clamped in a borehole to record seismic energy originating from various locations at the surface. F o r t h i s survey, a Vibrometric 8-level geophone tool was deployed in three deviated boreholes in multiple survey configurations using dynamite and Vibroseis sources. This comprised near and far source offsets as well as a Vibroseis walkaway (4Q66W3 only). Downhole receivers were spaced every $5 \mathrm{~m}$ and measured seismic waves on three orthogonal geophones. Preliminary processing of the zero-offset dynamite VSP data from 4Q66W3 included bandpass and notch filtering to remove electrical and background noise. This step has revealed reflections potentially resulting from the lithology, shallow-dipping faults or contacts with nearby ore lenses. Some ore lenses of the Callinan and 777 mines near 4Q66W3 have been completely removed. However, the morphology of the lens is preserved and the backfilled low-density material provides a contrast in seismic impedance strong enough to produce a reflection. Further processing will refine the previously-mentioned filters, rotate each component and remove the down-going waves, enhancing the reflections. Comparisons of all data for each borehole will aid interpretation, since multiple sources and offsets were used. Finite difference synthetic shot gathers, generated using a borehole-based 3D geological model, will be compared with the field VSP data sets to determine the origins of the reflections. 


\title{
Appendix E - Abstract: PDAC-SEG Student Minerals Colloquium 2014, Toronto
}

\section{VMS ore lens imaging and 3D finite difference modeling using vertical seismic profiles from Flin Flon, MB}

\author{
DM Melanson ${ }^{1}$ DJ White ${ }^{2}$ C Samson ${ }^{1}$ G Bellefleur ${ }^{2}$ E Schetselaar ${ }^{2}$ \\ ${ }^{1}$ Department of Earth Sciences, Carleton University, Ottawa, Ontario; ${ }^{2}$ Natural Resources \\ Canada, Ottawa, Ontario;
}

One of the main drivers of recent geophysical research in mineral exploration is to explore and define targets at greater depths. Seismic methods, including Vertical Seismic Profiling (VSP), currently provide the most powerful potential means to do so. In October 2006, three-component VSP data using dynamite and vibroseis sources were acquired from three deviated wells in the Flin Flon mining camp as part of a larger 3D seismic survey. These VSP data potentially contain a reflection signature of the $85.5 \mathrm{Mt}$ Flin Flon-Callinan-777 VMS ore system. From the many drill records, surficial maps and seismic data, a 3D voxel model of the local geology and known ore lenses has been built, which can be used in 3D finite difference-modeled simulations of the VSP surveys. The number of geological units partitioning the 3D voxel model is increased incrementally to study the effects on seismic response of massive sulfide ore and major rock units. The simulations are jointly visualized with the VSP data to aid interpretation. 


\title{
Appendix F - Abstract: Advances in Earth Sciences Research Conference 2014, Ottawa
}

\section{IMAGING AND 3D FINITE DIFFERENCE MODELING OF VMS ORE LENSES USING VERTICAL SEISMIC PROFILES FROM FLIN FLON, MB}

\author{
Melanson, D.M. ${ }^{1}$, White, D.J. ${ }^{2}$, Samson, C. ${ }^{1}$, Bellefleur, G. ${ }^{2}$ and Schetselaar, E. ${ }^{2}$ \\ ${ }^{1}$ Department of Earth Sciences, Carleton University, Ottawa, Ontario; \\ ${ }^{2}$ Natural Resources Canada, Ottawa, Ontario;
}

One of the main drivers of recent geophysical research in mineral exploration is to explore and define targets at greater depths. Seismic methods, including Vertical Seismic Profiling (VSP), currently provide the most powerful potential means to do so. In October 2006, three-component VSP data using dynamite and vibroseis sources were acquired from three deviated wells in the Flin Flon mining camp as part of a larger 3D seismic survey. These VSP data potentially contain a reflection signature of the $85.5 \mathrm{Mt}$ Flin Flon-Callinan-777 VMS ore system. From the many drill records, surficial maps and seismic data, a 3D voxel model of the local geology and known ore lenses has been built, which can be used in 3D finite difference-modeled simulations of the VSP surveys. The number of geological units partitioning the $3 \mathrm{D}$ voxel model is increased incrementally to study the effects on seismic response of massive sulfide ore and major rock units. The simulations are jointly visualized with the VSP data to aid interpretation. 


\section{Appendix G - Abstract: Geological Association of Canada - Mineralogical Association of Canada Joint Annual Meeting 2014, Fredericton}

\section{VMS ore lens imaging and 3D finite difference modeling using vertical seismic profiles from Flin Flon, $\mathrm{MB}$}

DM Melanson $^{1}$ DJ White ${ }^{2}$ C Samson ${ }^{1}$ G Bellefleur ${ }^{2}$ E Schetselaar ${ }^{2}$

${ }^{1}$ Department of Earth Sciences, Carleton University, Ottawa, Ontario; ${ }^{2}$ Natural Resources Canada, Ottawa, Ontario;

One of the main drivers of recent geophysical research in mineral exploration is to explore and define targets at greater depths. Seismic methods, including Vertical Seismic Profiling (VSP), currently provide the most powerful potential means to do so. In October 2006, three-component VSP data using dynamite and vibroseis sources were acquired from three deviated wells in the Flin Flon mining camp as part of a larger 3D seismic survey. These VSP data potentially contain a reflection signature of the $85.5 \mathrm{Mt}$ Flin Flon-Callinan-777 VMS ore system. From the many drill records, surficial maps and seismic data, a 3D voxel model of the local geology and known ore lenses has been built, which can be used in 3D finite difference-modeled simulations of the VSP surveys. The number of geological units partitioning the 3D voxel model is increased incrementally to study the effects on seismic response of massive sulfide ore and major rock units. The simulations are jointly visualized with the VSP data to aid interpretation. 


\title{
Appendix H - Abstract: Society of Economic Geologists Annual Meeting 2014, Keystone
}

\author{
Delineation of VMS ore lenses in Flin Flon, MB, Canada using vertical seismic \\ profiles and 3D finite difference modeling \\ DM Melanson ${ }^{1}$ DJ White ${ }^{2}$ C Samson ${ }^{1}$ G Bellefleur ${ }^{2}$ E Schetselaar $^{2}$ \\ ${ }^{1}$ Department of Earth Sciences, Carleton University, Ottawa, Ontario; ${ }^{2}$ Natural Resources \\ Canada, Ottawa, Ontario;
}

One of the main drivers of recent geophysical research in mineral exploration is the desire to investigate and define targets at greater depths. Seismic methods, including vertical seismic profiling (VSP), currently provide a means of achieving this goal. In October 2006, three-component VSP data using dynamite and Vibroseis sources were acquired in three deviated wells in the Flin Flon mining camp as part of a larger 3D seismic survey. The Flin Flon mining camp is located in northern Manitoba, Canada within the Amisk collage of the 1.92-1.83 Ga Flin Flon-Glennie complex, which was obducted during the collisional stages of the Trans-Hudson orogeny. The VSP data appear to have an observable reflection response from the 85.5 Mt Flin FlonCallinan-777 VMS ore system. This system comprises pyrrhotite, chalcopyrite and sphalerite-rich ore lenses are hosted within altered rhyolites of the Millrock member, which in turn lies between mafic volcanic and volcaniclastic rocks of the footwall and hanging wall. This region has been subjected to greenschist metamorphic facies and several deformation events. Boundaries between rock units and the massive sulfide ore provide strong contrasts in acoustic impedance from which seismic reflections will originate.

The Flin Flon mining camp has been extensively mapped, drilled, mined and targeted by geophysical surveys for decades, leading to a wealth of multidisciplinary geoscience data. From these data, several voxel models were constructed, which can be used in 3D finite difference-modeled simulations of the VSP surveys. The voxel models are populated with elastic rock properties that have been determined from physical rock properties measured using in-situ sonic logs and laboratory measurements on core samples from the boreholes used for VSPs in this study. The number of distinct geological units used in the 3D voxel model was increased incrementally to determine the effects on seismic response of major rock units and massive sulfide ore. The outputs of this simulation method were synthetic VSP shot-gathers, which capture the particle velocity at receiver stations. In addition, the simulated propagation and scattering of the seismic wavefields can be visualized in $2 \mathrm{D}$ and $3 \mathrm{D}$ using the output from the simulations taken at sequential time steps. These synthetic shot-gathers were found to be directly comparable to the VSP field data. By integrating the modeled results into the interpretation process, we were able to identify and characterize the response from the mine horizon and from the massive sulfide ore in the VSP field data. 